\title{
Hyperelliptic Curves from the Geometric and Algebraic Perspectives
}

\author{
by \\ Colin Weir, B.Math \\ A thesis submitted to \\ the Faculty of Graduate Studies and Research \\ in partial fulfillment of \\ the requirements for the degree of \\ Master of Science, \\ School of Mathematics and Statistics \\ Ottawa-Carleton Institute for Mathematics and Statistics \\ Carleton University \\ Ottawa, Ontario, Canada \\ (C) Copyright 2008, \\ Colin Weir
}




$\begin{array}{ll}\text { Library and } & \text { Bibliothèque et } \\ \text { Archives Canada } & \text { Archives Canada } \\ \begin{array}{l}\text { Published Heritage } \\ \text { Branch }\end{array} & \begin{array}{l}\text { Direction du } \\ \text { Patrimoine de l'édition }\end{array} \\ \begin{array}{l}\text { 395 Wellington Street } \\ \text { Ottawa ON K1A ON4 }\end{array} & \begin{array}{l}\text { 395, rue Wellington } \\ \text { Cttawa ON K1A ON4 } \\ \text { Canada }\end{array} \\ \end{array}$

Your file Votre référence ISBN: 978-0-494-44146-6

Our file Notre référence

ISBN: 978-0-494-44146-6

NOTICE:

The author has granted a nonexclusive license allowing Library and Archives Canada to reproduce, publish, archive, preserve, conserve, communicate to the public by telecommunication or on the Internet, loan, distribute and sell theses worldwide, for commercial or noncommercial purposes, in microform, paper, electronic and/or any other formats.

The author retains copyright ownership and moral rights in this thesis. Neither the thesis nor substantial extracts from it may be printed or otherwise reproduced without the author's permission.
AVIS:

L'auteur a accordé une licence non exclusive permettant à la Bibliothèque et Archives Canada de reproduire, publier, archiver, sauvegarder, conserver, transmettre au public par télécommunication ou par l'Internet, prêter, distribuer et vendre des thèses partout dans le monde, à des fins commerciales ou autres, sur support microforme, papier, électronique et/ou autres formats.

L'auteur conserve la propriété du droit d'auteur et des droits moraux qui protège cette thèse. $\mathrm{Ni}$ la thèse ni des extraits substantiels de celle-ci ne doivent être imprimés ou autrement reproduits sans son autorisation.
In compliance with the Canadian Privacy Act some supporting forms may have been removed from this thesis.

While these forms may be included in the document page count, their removal does not represent any loss of content from the thesis.

$$
\text { Canada' }
$$

Conformément à la loi canadienne sur la protection de la vie privée, quelques formulaires secondaires ont été enlevés de cette thèse.

Bien que ces formulaires aient inclus dans la pagination, il n'y aura aucun contenu manquant. 
To my great relief 


\section{Abstract}

We examine hyperelliptic curves from two perspectives: a geometric perspective and an algebraic perspective. In both cases, our objective is to describe in detail the necessary concepts to understand the definition and properties of a hyperelliptic curve. Geometrically, we consider both affine and projective curves. We explore maps and functions on curves, and then investigate the local properties of curves, their local rings and valuations. Using tangent spaces, we define and describe both rational and regular differential forms. Lastly, using differential forms and divisors we compute the genus of a hyperelliptic curve. Algebraically, we describe hyperelliptic curves by examining function fields. We explore and explicitly compute the places of a hyperelliptic function field. This is accomplished by considering the absolute values and completions associated to places. Using the algebraic definitions of differential forms and their divisors, we compute the genus of a hyperelliptic function field. 


\section{Acknowledgments}

I would like to thank my supervisor Dr. Paul Mezo for his diligence, patience, and occasional words of encouragement... they were appreciated. I would also like to thank him for pushing me to a level that I could not have reached otherwise and giving me the gift of knowing my potential.

I'd also like to thank Dr. Daniel Panario and Dr. Monica Nevins for their time, energy, and helpful comments.

Lastly, I'd like to thank to all those who stood by me during my difficulties with this (especially the one who sat by me). I could not have done it without you. 


\section{Contents}

Abstract

1 Introduction 1

2 The Geometric Perspective 11

2.1 Affine and Projective Spaces . . . . . . . . . . . . . . . 11

2.1.1 Affine and Projective Sets . . . . . . . . . . . . 11

2.1.2 Homogenization and Dehomogenization . . . . . . . . . . 18

2.1 .3 Regular Functions . . . . . . . . . . . . . . . . . . . . 21

2.1 .4 Regular Maps . . . . . . . . . . . . . . . . . . 25

2.1 .5 Rational Functions . . . . . . . . . . . . . . . . . 29

2.1 .6 Rational Maps . . . . . . . . . . . . . . . . . . . 31

2.2 Tangent lines . . . . . . . . . . . . . . . . . . . . . . . . . . . . . . . . . 39

2.3 Valuations and the Local Ring at a Point . . . . . . . . . . . . 46

2.4 Nonsingular Models . . . . . . . . . . . . . . . . . . . . . . 62

2.5 Divisors of Points . . . . . . . . . . . . . . . 68

2.6 Differential Forms . . . . . . . . . . . . . . . . . 74

2.6.1 Regular Differential Forms . . . . . . . . . . . . . 77

2.6.2 Rational Differential Forms and their Divisors . . . . . . . 85

2.7 The Genus of a Curve $\ldots \ldots \ldots \ldots$

$\begin{array}{lll}3 & \text { The Algebraic Perspective } & 99\end{array}$ 
3.1 Places . . . . . . . . . . . . . . . . . . . . . . . . 100

3.2 Valuations, Absolute Values and Completions . . . . . . . . . 111

3.2 .1 Discrete Valuations . . . . . . . . . . . . . . . . . . . 112

3.2 .2 Absolute Values . . . . . . . . . . . . . . . . . . . . . . 115

3.2 .3 Completions . . . . . . . . . . . . . . . . . . . . . . . 118

3.2 .4 The Completions of $K(x) \ldots \ldots \ldots \ldots \ldots$

3.3 Extensions of Function Fields . . . . . . . . . . . . . . . . 128

3.3.1 Places and Extensions . . . . . . . . . . . . . . . . 129

3.3.2 The Relative Degrees of Places _. . . . . . . . . . . 134

3.3.3 The Ramification Index of Places . . . . . . . . . . . 136

3.3.4 Basic Galois Theory . . . . . . . . . . . . . . . . . . 140

3.4 Completions of Algebraic Extensions . . . . . . . . . . . . . . 141

3.5 The Places of a Quadratic Extension of $K(x) \ldots \ldots \ldots$

3.6 Further Properties of the Ramification Index and Relative Degree . . 154

3.7 Further Properties of the Places of a Quadratic Extension of $K(x) \ldots 163$

3.8 Divisors . . . . . . . . . . . . . . . . . . . . . 167

3.9 Divisors and Extensions: Norm and Conorm . . . . . . . . . 174

3.10 Differential Forms . . . . . . . . . . . . . . . . . . . . . . . . 181

3.11 The Different and The Riemann Hurwitz Genus Formula . . . . . . . 201

4 Algebra and Geometry $\quad 206$

4.1 Hyperelliptic Curves . . . . . . . . . . . . . . 206

5 Conclusions $\quad 210$

$\begin{array}{ll}\text { Bibliography } & 212\end{array}$ 


\section{Chapter 1}

\section{Introduction}

Hyperelliptic curves, while utilized in various branches of mathematics, are perhaps most well known for their applications to cryptography. Koblitz [8] first suggested using hyperelliptic curves for cryptographic purposes. Since then, the cryptographic applications of hyperelliptic curves continue to be very well studied [1]. The use of elliptic curves, which may be defined as a particular class of hyperelliptic curves, has become very popular in cryptography.

Most of the applications of hyperelliptic curves are a result of their associated group structure. One may form a group on the divisors of a hyperelliptic curve. In the case of an elliptic curve, this group may be formulated solely in terms of the points on the curve. In either case, the size of the associated group is in direct relation to the number of points on the corresponding curve. However, enumerating the points on a hyperelliptic curve (or calculating the order of the associated group) is not a trivial task [15]. This fact gives added interest to the study of hyperelliptic curves and added security to the cryptosystems that employ them.

Regardless of its formulation, the properties of the group of a hyperelliptic curve are utilized in numerous applications. One may consider the random variable representing the order of the group of the curve [11]. This random variable is completely dependent on the choice of hyperelliptic curve, yet its value is difficult to verify. As 
such, the possible orders of the elements of the group are difficult to verify. This fact allows for the group structure of hyperelliptic curves to be used in various pseudorandom number generators [10]. Taking powers of elements of the group of a hyperelliptic curve may be used to create seemingly unpredictable sequences.

The variability in the order of the group of hyperelliptic curves is utilized in different factoring algorithms [11]. By choosing various hyperelliptic curves, one is probabilistically choosing random groups. Using these random groups, one may perform probabilistic checks for the primality of a number. The variability in the order of the group gives some added efficiency to various primality testing algorithms.

From the perspective of algebraic geometry, one may consider the modulus of hyperelliptic curves. One may partition hyperelliptic curves into birationally equivalent classes [3]. Each of these classes is then considered as a unique element. With these classes as elements, one may create a space and apply the principles of algebraic geometry to this new space. In this space one may then define closed and open sets and again investigate any properties of interest, including differential forms and genus; concepts we will introduce shortly.

Hyperelliptic curves also appear in other branches of mathematics including number theory, analysis, and algebraic topology. However, regardless of the application or perspective, most of the existing literature presupposes a basic knowledge of hyperelliptic curves. Hyperelliptic curves are fundamentally objects of algebraic geometry, yet as we have seen, there are many areas apart from algebraic geometry which examine hyperelliptic curves. With this wide range of perspectives come various definitions and descriptions of hyperelliptic curves. This can result in a great deal of confusion for inexperienced readers.

Quite often in practical applications, a naive definition of a hyperelliptic curve is used; the definition of a hyperelliptic curve is taken to be the zero set of a polynomial $y^{2}-\varphi(x)$ for some non-constant polynomial $\varphi(x)$ together with a "point at infinity". This naive definition provides a good way to visualize a hyperelliptic curve. As an 
example, the graph of some $y^{2}-\varphi(x)$ is given over the real plane in Figure 1.1.

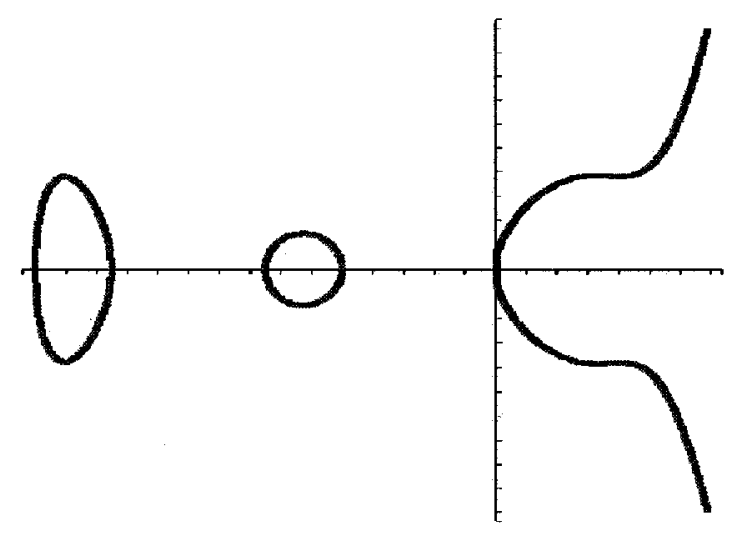

Figure 1.1: The curve $y^{2}+x(x+1)(2 x+3)(2 x+5)(x+3)\left(x^{2}+x+1\right)=0$ over $\mathbb{R}^{2}$

However, this definition does not tell the whole story. One properly defines a hyperelliptic curve as follows.

Definition 1.1 ([1] p.73) Let $K$ be a field with characteristic not equal to 2. Let $C$ be an irreducible nonsingular projective curve of genus $g \geq 1$. If the rational function field $K(C)$ of $C$ is a quadratic extension of $K(x)$ for some element $x$ transcendental over $K$ then $C$ is called a hyperelliptic curve.

Unravelling this definition is a rather daunting task for the beginner, and it is our main goal. We will approach this task from two perspectives; a geometric perspective and an algebraic perspective. We have devoted a chapter to each of these two topics with an additional chapter devoted to the partial unification of these two perspectives. By the final chapter, we will have all the tools necessary to properly define and describe hyperelliptic curves.

This work is geared towards a reader with an undergraduate understanding of mathematics who is not necessarily well-versed in either algebraic geometry or function fields. As a result, this work also serves as an introduction of both topics. This is especially important, as nearly all texts in these subjects are geared to a graduate 
student audience at least. Futhermore, even though much is known about hyperelliptic curves, all texts in either algebraic geometry or function fields devote little time to them in an effort to maintain their generality. Consequently, many of the results, and how they pertain to hyperelliptic curves, are glossed over or disregarded.

In an effort to serve as an introduction to hyperelliptic curves, we will make explicit the consequences and details of the general theory. To this end, we will keep the theorems and their proofs as general as possible, and use examples to clarify how the general theory applies to the case of hyperelliptic curves. To further this aim, our work is nearly self-contained. This is also significant as the introductory material on these subjects leaves an abundant amount of details to the reader.

This work also contains information that is quite possibly not found in any other text. Not only are the demonstrations of the general theory to the case of hyperelliptic curves original, the path which we take through that theory is novel as well. A conscious effort has been made to avoid certain advanced topics which other authors typically include. This results in a more approachable document with some atypical proofs. Furthermore, this work contains a complete classification and computation of all the places of a hyperelliptic function field. The theorems used to accomplish this were compiled from several sources, however this achievement does not appear to be found in any other text. This result is especially important as these places are the building blocks of the associated group structure of a hyperelliptic curve.

We hope that achieving these objectives will result in a document of some merit. With the numerous applications to hyperelliptic curves, many have taken interest in studying them. However, there is no document to serve as an introduction to hyperelliptic curves from either of the perspectives we are considering. As such, this work should be of value to a variety of people wishing to pursue the theory of hyperelliptic curves and its applications.

We begin with the geometric perspective of our pursuit. In Chapter 2 we will always assume $K$ to be an algebraically closed field. Most of our examples will be 
about plane curves defined by the equation $y^{2}=\varphi(x)$. As we will see, we may infer many of the properties of a hyperelliptic curve by examining this one.

In Section 2.1 we provide the building blocks of algebraic geometry. We define affine and projective closed sets as zero sets of certain polynomial equations, which includes the definition of a plane curve. We then define some fundamental properties of closed sets such as irreducibility and nonsingularity. Moreover, we demonstrate how a projective plane curve has a covering isomorphic to a set of affine plane curves. These are called the affine pieces of a projective curve. When we are discussing the properties of points on a projective plane curve we often consider an affine piece containing that point to simplify our discussion.

In Section 2.1 we also define maps between closed sets. We explore several types of maps between affine and projective sets. In particular, we explore maps that relate affine and projective sets called homogenization and dehomogenization. We go on to describe what is meant by a birational equivalence and an isomorphism between curves. An isomorphism is a stronger equivalence than a birational equivalence. All the properties of interest to us will be invariant under isomorphism and most will be invariant under birational equivalence. Hence, to describe the properties of a hyperelliptic curve as in Definition 1.1, we may often describe them in terms of a projective plane curve to which the hyperelliptic curve is birationally equivalent. Again, this will simplify our discussion.

We also explore other functions on closed sets. We introduce the ring of regular functions on a plane curve. In the affine setting, this turns out to be the polynomial functions on the curve, but in the projective setting the only regular functions are constant functions. This motivates us to consider a wider class of functions, namely rational functions. The rational functions on a curve form a field. Moreover the fields of rational functions of two birationally equivalent curves are isomorphic. This, once again, allows us to consider plane curves to which hyperelliptic curves are birationally equivalent. 
In Section 2.2 we define what is meant by a tangent line to a curve. We will show how this is a generalization of the intuitive definition of a tangent line. The equation of a tangent line will become useful in the following section. We also associate a closed set to tangent lines and later consider the linear functions on this set. The set of these functions is called the dual of the tangent space. The dual space is a basic ingredient in the definition of a differential form.

Section 2.3 is dedicated to the local properties of points on a curve. It begins with the definition of the local ring at a point. A local ring at a point consists of the rational functions defined at that point. Using tangent lines, we show how each local ring at a nonsingular point has a unique maximal principal ideal and it is invariant under isomorphism. This invariance allows us to describe the local rings of a hyperelliptic curve in terms of a planar one. We show how the ideals of local rings provide us with functions called discrete valuations. Discrete valuations are functions from the function field of the curve to the integers. They provide a measure for the multiplicity of a zero or a pole of a rational function at that point.

In Section 2.4 we describe how all plane curves are birationally equivalent to nonsingular curves (not necessarily planar). The proof of this result is rather long and constructive by nature. As it does not really give any further insight into hyperelliptic curves, we give only a brief description of the proof and instead explore some of its consequences. We make more explicit why it is nearly sufficient to examine planar curves when our true goal is to examine hyperelliptic curves which are not necessarily planar.

In Section 2.5 we define an object to record the information about the zeros and poles of a rational function; this object is called a divisor. We then form divisor classes, whereby two divisors are equivalent if they differ only by the divisor of a rational function. While the zeros and poles of a rational function are not invariant under birational maps, the number of them is. So in a manner of speaking, divisor classes provide a means to measure global rationality; something invariant under 
birational equivalence. This will provide a means to describe the zeros and poles of a differential form.

In Section 2.6 we introduce differential forms. We build up to the definition of a regular differential form by defining various maps from rational functions to the duals of tangent spaces. We then give the definition of a rational differential form. Rational differential forms are functions and so using Sections 2.3 and 2.5 we may assign divisors to them. We then show how all rational differential forms are in the same divisor class. Using divisors we may then determine which rational differential forms are regular, in the sense that they have no poles.

Using the information gathered in the previous sections we are able to describe the genus of a hyperelliptic curve. Hyperelliptic curves over the complex numbers are equivalent to analytic objects called compact Riemann surfaces [12]. Here one may show that a hyperelliptic curve is equivalent to a multiple "holed" torus. Analytically and topologically, the number "holes" of a surface is called the genus. This begin said, the purpose of Section 2.7 is to provide a rigorous definition of the genus of a curve; one in terms of differential forms. We show that the regular differential forms form a vector space. After doing so, we demonstrate how the vector space of regular differential forms is isomorphic to a vector space associated to the divisor of a regular differential form. The genus is then defined as the dimension of these vector spaces. To compute the genus, we prove that we need only find the dimension of the divisor space of any rational differential form. We do this explicitly for the case of hyperelliptic curves.

In Chapter 3 we take an alternative approach to hyperelliptic curves. As birationally equivalent curves have isomorphic function fields, we disregard the geometric properties of hyperelliptic curves and turn our attention towards quadratic extensions of function fields, as in Definition 1.1. This perspective allows us to drop the condition that our field $K$ be algebraically closed. Moreover, without the geometric constraints, we are able to give a more comprehensive account of the local properties 
of a hyperelliptic curve.

As previously mentioned in Definition 1.1, the rational function field of a hyperelliptic curve $K(C)$ is a quadratic extension of $K(x)$ and all such extensions are isomorphic to $K(x)[y] /\left(y^{2}-\varphi(x)\right)$ which we will abusively denote as $K(x, y)$. While we will try to keep the theorems and definitions as general as possible, our examples will focus on extensions of this form. Thus, much of our work on hyperelliptic function fields will take place in the examples. Some of our later results, while true in general, are only proved for case of quadratic extensions.

We begin by investigating the valuation rings of function fields. As mentioned in the previous chapter, discrete valuation rings have maximal principal ideals. In this chapter we will call these ideals "places". In the case of an algebraically closed field, the points on a non-singular projective curve are in one-to-one correspondence with the places. As such, completely describing the places of a quadratic extension yields a complete description of the points of a hyperelliptic curve.

Our approach to finding the places of $K(x, y)$ is analogous to the following geometric point of view. Consider the projection from the curve $y^{2}-\varphi(x)$ down to the $x$-axis. We notice that cach point on the $x$-axis has at most two points that project to it. This may be visualized by examining Figure 1.1. A similar notion holds true for places. Let $P$ be a place of $K(x)$. Then there exists at most two places of $K(x, y)$ that contain $P$ as a subset. Moreover, every place of $K(x, y)$ contains exactly one place $P$ of $K(x)$. We will prove this fact and use it to find all the valuation rings of $K(x, y)$.

In Section 3.1 we make explicit the definition of places $P$ and valuation rings $\mathcal{O}_{P}$ over an arbitrary function field. We will then go on to see that their properties are similar to those defined over curves in Section 2.3. Using these properties, we are then able to give a complete list of the places of the rational function field $K(x)$.

In Section 3.2 we move on to define the discrete valuation associated to a place. Using discrete valuations we may define yet another measure of the zeros and poles of 
a rational function, namely an absolute value. To each absolute value we may define a completion of its underlying field. In addition, we will see that places, valuation rings, absolute values, and completions are all in one-to-one correspondence with each other. Thus, finding the unique completions of $K(x, y)$ will give us all the valuation rings and places.

We need more machinery to describe the completions of a quadratic extension. In Section 3.3 we describe further the relationship between places $P \subset K(x)$ and the places $P^{\prime} \subset K(x, y)$. We define two important properties; the ramification index and the relative degree. The ramification index is a positive integer $e$ which relates the valuation of the place $P \subset K(x)$ with that of the place $P^{\prime} \in K(x, y)$ where $P \subset P^{\prime}$. The relative degree $f$ is a positive integer that relates the residue fields $\mathcal{O}_{P} / P$ and $\mathcal{O}_{P^{\prime}} / P^{\prime}$ where $\mathcal{O}_{P}$ and $\mathcal{O}_{P^{\prime}}$ are the valuation rings of the places $P$ and $P^{\prime}$ respectively. In Section 3.4 we work towards an important theorem describing the possible absolute values of $K(x, y)$. We will see that a finite extension of a complete field has a unique absolute value. As a result, we are able to completely describe the absolute values and thus the valuations at each of the places.

With this information we are able to describe all the places of $K(x, y)$ in Section 3.5. We separate our description into four cases based upon the ramification indices. Using the results of the previous section we also prove that our list of the places of $K(x, y)$ is complete.

In Section 3.6, we further explore the properties of the ramification index and relative degree. Reusing the Galois theory introduced Section 3.3, we may prove that the ramification indices and relative degrees of certain places are equal. This will will aid in our proof of an important theorem. For a given place $P \in K(x)$, we let $r$ denote the number of places $P^{\prime} \in K(x, y)$ such that $P^{\prime} \supset P$, and then prove that $r e f=2$. With this important result we are able to compute the ramification indices and relative degrees of every place of $K(x, y)$ in Section 3.7. We again partition our examination into four cases and dedicate a subsection to each. 
With the results of the previous sections, we may describe the zeros and poles of a rational function at places instead of points. Just as we did for curves, we use divisors to record this information. Section 3.8 introduces this subject. We then take the study of divisors further. In Section 3.9, we explore how divisors of $K(x, y)$ relate to those of $K(x)$. In particular we define two maps between the divisors of $K(x, y)$ and those of $K(x)$; namely the Norm and Conorm functions. These functions will ultimately allow us to relate the genus of $K(x)$ to that of $K(x, y)$; something we could not do in Chapter 2.

Imitating Chapter 2, we will use divisors and differential forms to calculate the genus. We begin Section 3.10 by giving an algebraic defintion of a differential form, in keeping with the geometric definition. Then as we did in Chapter 2, we define valuations and divisors of differential forms. We give a definition of regularity of a differential form in terms of divisors. This allows us to then define and calculate the genus of a given hyperelliptic function field. Furthermore, because of our work on divisors and extensions, we are also able to show the converse via the Riemann Hurwitz Genus Formula. Given that a hyperelliptic function field has genus $g$, we show that it is isomorphic to $K(x, y)$ where $y^{2}=\varphi(x)$ and the polynomial $\varphi(x)$ has degree $2 g+1$ or $2 g+2$.

Finally, in Chapter 4 we relate our work on function fields back to curves. We summarize our conclusions and make explicit the relationship between the points on a nonsingular curve and the places of a function field. We give the definition of a hyperelliptic curve, and combining the results of the previous two chapters, we will finally be able to explicitly describe a hyperelliptic curve. We will prove that the genus of a hyperelliptic curve is equal to the genus of its function field. Moreover, we show that all hyperelliptic curves are birationally equivalent to the projective closures of the affine curves defined by the equation $y^{2}=\varphi(x)$. 


\section{Chapter 2}

\section{The Geometric Perspective}

Throughout this chapter $K$ will represent an arbitrary algebraically closed field with characteristic not equal to 2 . We will let $K[x]$ denote the ring of polynomials with coefficients in $K$ and $K(x)$ denote the field of fractions of $K[x]$, namely the field of rational functions with coefficients in $K$. The results of this chapter are founded upon [16] and [4].

\subsection{Affine and Projective Spaces}

In this section we give an overview of the building blocks of algebraic geometry. We describe closed and open sets and some of their basic properties. Descriptions will be given for closed subsets of both affine and projective spaces. We also describe different types of maps between open and closed sets in both the affine and projective setting. These maps are built upon the rational and regular functions on a closed set. These functions will play a fundamental role in describing closed sets as we move forward in later sections.

\subsubsection{Affine and Projective Sets}

We begin with the definition of affine space. 
Definition 2.1.1 Let $\mathbb{A}^{n}=\left\{\left(k_{1}, \ldots, k_{n}\right) \mid k_{i} \in K, i=1, \ldots, n\right\}$ be the set of $\mathrm{n}$-tuples of $K$. We call $\mathbb{A}^{n}$ the $n$-dimensional affine space. More specifically, we call $\mathbb{A}^{1}$ the affine line and $\mathbb{A}^{2}$ the affine plane. In all cases, we refer to the elements of $\mathbb{A}^{n}$ as points.

Definition 2.1.2 ([16] p.23) An affine closed subset $A \subseteq \mathbb{A}^{n}$ is a set consisting of all the common zeros of a set of polynomial functions with coefficients in $K$. We will often refer to this a closed set for brevity.

Throughout this work we will let $t$ represent the set of variables $t_{1}, \ldots, t_{n}$. In keeping with this we will let $a(t)$ represent a polynomial function $a$ in the $n$ variables $t_{1}, \ldots, t_{n}$. However, when specifically referring to the affine plane we will often use the traditional coordinates $(x, y)$.

Definition 2.1.3 Let $A$ be an affine closed set consisting of the common zeros of $a_{1}(t), \ldots, a_{m}(t)$. We say that $A$ is defined by the polynomials $a_{1}(t), \ldots, a_{m}(t)$.

Notice that the definition of a closed set $A$ (Definition 2.1.2) does not require that $A$ be defined by a finite set of polynomials. However, if $A$ is defined by an infinite set of polynomials, we will see later on (Theorem 2.1.32) that there exists a finite set of polynomials which also defines $A$.

Definition 2.1.4 Let $C$ be a closed subset of $\mathbb{A}^{2}$. If $C$ is defined by one non-constant polynomial then $C$ is called an affine plane curve.

A lot of our focus will be on affine plane curves. We will however maintain a higher level of generality for the majority of this section and typically use affine plane curves in our examples.

Definition 2.1.5 ([16] p.23) Let $A \subset \mathbb{A}^{n}$ be a closed set. We say that a subset $U \subset A$ is open if its compliment $A \backslash U$ is closed. We also define a neighbourhood of a point $p \in A$ as any open set containing $p$. 
We wish to examine what open sets look like for affine plane curves. To do so, we need the following lemma.

Lemma 2.1.6 ([16] p.2) Let $c(x, y) \in K[x, y]$ be an irreducible polynomial and let $g(x, y) \in K[x, y]$ be an arbitrary polynomial. If $g(x, y)$ is not divisible by $c(x, y)$ then the system $g(x, y)=c(x, y)=0$ has only finitely many solutions.

Proof. Suppose that $x$ appears in $c(x, y)$ with degree greater than 0 . We begin by viewing $c(x, y)$ and $g(x, y)$ as elements of $K(y)[x]$ where $K(y)$ denotes the rational functions in $y$. We first note that $c(x, y)$ remains irreducible in this ring. Indeed, if $c(x, y)$ were to split into factors, then after multiplying by a common denominator $g(y) \in K[y]$ we would obtain a relation contradicting the irreducibility of $c(x, y)$ in $K[x, y]$. Using the same reasoning $c(x, y)$ does not divide $g(x, y)$ in the new ring $K(y)[x]$. Hence, by the Extended Euclidean Algorithm ([2] p.276), there exists elements $u_{1}^{\prime}(x, y), u_{2}^{\prime}(x, y) \in K(y)[x]$ such that

$$
u_{1}^{\prime}(x, y) c(x, y)+g(x, y) u_{2}^{\prime}(x, y)=1
$$

Multiplying this equation by a common denominator $a(y) \in K[y]$, we obtain that

$$
u_{1}(x, y) c(x, y)+g(x, y) u_{2}(x, y)=a(y)
$$

where $u_{1}(x, y)=a(y) u_{1}^{\prime}(x, y) \in K[x, y]$ and $u_{2}(x, y)=a(y) u_{1}^{\prime}(x, y) \in K[x, y]$. It follows that if $c\left(p_{1}, p_{2}\right)=g\left(p_{1}, p_{2}\right)=0$ then $a\left(p_{2}\right)=0$. Consequently, there are only finitely many values $p_{2}$ such that this is the case. Furthermore, for each value, $c\left(x, p_{2}\right)$ is not identically 0 (otherwise $y-p_{2}$ would be a factor of $c(x, y)$ ), and thus has only finitely many solutions. Thus the system $g(x, y)=c(x, y)=0$ has only finitely many solutions.

Suppose that $x$ does not appear with degree greater then 0 . Since $K$ is algebraically closed the irreducibility of $c(x, y)$ implies that $c(x, y)=d y-b$ for some 
$b, d \in K$. As such, the system $g(x, y)=c(x, y)=0$ is reduced to $g\left(x, d^{-1} b\right)=0$ which has only finitely many solutions.

We examine the possible open subsets of a plane affine curve defined by an irreducible polynomial.

Example 2.1.7 Let $C$ be an affine plane curve defined by an irreducible polynomial $c(x, y)$. Let $U$ be a closed set on $C$ defined by the polynomial $u(x, y)$. The points of $U$ are exactly those on $C$ that satisfy $u(x, y)=0$, i.e. the common zeros of $u(x, y)$ and $c(x, y)$. There are two cases to consider. If $u(x, y)$ is a multiple of $c(x, y)$ then $C$ is the set of $c(x, y)=0$ which proves that $C$ is a closed set and the empty set is an open subset of $C$. If $u(x, y)$ is not a multiple of $c(x, y)$ then the irreducibility of $c(x, y)$ implies that there are at most finitely many common zeros. This means that $U$ consists of a finite set of points. Thus the open set $C \backslash U$ is simply the set $C$ minus finitely many points.

Proposition 2.1.8 The arbitrary (possibly infinite) intersection of closed sets is closed.

Proof. Let $C_{\alpha}$ be a collection of closed sets defined by $c_{\alpha, \beta}(t)$. Then $\cap_{\alpha} C_{\alpha}$ is defined by the common zeros of the $c_{\alpha, \beta}(t)$ and thus a closed set.

Proposition 2.1.9 ([16] p.23) The union of a finite number of closed sets is closed. Proof. By induction, it is enough to show that the union of two closed sets is closed. Let $A=A_{1} \cup A_{2}$ where $A_{1}$ and $A_{2}$ are closed sets defined by the polynomials $f_{1}(t), f_{2}(t), \ldots$ and $g_{1}(t), g_{2}(t), \ldots$ respectively. We will see that the set $S=\left\{f_{i}(t) g_{j}(t) \mid i=1,2, \ldots j=1,2, \ldots\right\}$ defines $A$. Indeed, any common zero of $f_{1}(t), f_{2}(m), \ldots$ and $g_{1}(t), g_{2}(t), \ldots$ is certainly a common zero of the polynomials of $S$. Conversely, if a point $p$ is a common zero of $S$, then without loss of generality we suppose that $p \notin A_{1}$. This implies that there exists an $f_{l}(t)$ such that $f_{l}(p) \neq 0$. Since $p$ is a common zero of a $f_{l}(p) g_{j}(p)=0$ for all $j=1,2, \ldots$ thus $g_{j}(p)=0$ for all $j=1,2, \ldots$ This means that $p \in A_{2}$ and consequently that $S$ defines $A$. 
Now that we have defined closed affine sets and seen some examples, we move on to discuss some properties that will help us classify different types of closed sets.

Definition 2.1.10 ([16] p.23) A closed set $A \subset \mathbb{A}^{n}$ is called reducible if there exist closed sets $A_{1}$ and $A_{2}$ such that $A=A_{1} \cup A_{2}$. A closed set that is not reducible is called irreducible.

Example 2.1.11 Let $C \subset \mathbb{A}^{2}$ be a closed set defined by $c(x, y)=y^{2}-\varphi(x)$ where $\varphi(x) \in K[x]$ is a square. This means that there exists a $g(x) \in K[x]$ such that $g(x)^{2}=\varphi(x)$. Consider the closed sets $C_{1}$ and $C_{2}$ defined by $c_{1}(x, y)=y+g(x)$ and $c_{2}(x, y)=y-g(x)$ respectively. Clearly $C_{1}, C_{2}$ are subsets of $C$. Moreover, since $c(x, y)=c_{1}(x, y) c_{2}(x, y)$ we have that $C=C_{1} \cup C_{2}$.

To describe the smoothness properties of points on a closed set we need to take derivatives. We will use the notation $\frac{\partial g}{\partial t_{i}}(t)$ to mean the formal partial derivative of the polynomial $g(t)$ with respect to the variable $t_{i}$ for any $i=1, \ldots, n$. With this being said, we move on to discuss singularities of a curve.

Definition 2.1.12 ([16] p.12) Let $C$ be an affine plane curve defined by $c(x, y)$. A point $p \in C$ is called singular or a singularity if $\frac{\partial c}{\partial x}(p)=\frac{\partial c}{\partial y}(p)=0$. A point $p \in C$ that is not singular is called nonsingular and a curve whose points are all nonsingular is called a nonsingular curve.

Example 2.1.13 Let $C \subset \mathbb{A}^{2}$ be a closed set defined by $c(x, y)=y^{2}-\varphi(x)$ where $\varphi(x) \in K[x]$. Exploring the possible singularities of $C$, we see that

$$
\frac{\partial c}{\partial x}(x, y)=-\varphi^{\prime}(x) \quad \text { and } \quad \frac{\partial c}{\partial y}(x, y)=2 y
$$

Now, $2 y=0$ implies that $\varphi(x)=0$ as $\operatorname{char}(K) \neq 2$. So $C$ has a singularity if and only if there exists a point $(0, p) \in C$ such that $\varphi(p)=\varphi^{\prime}(p)=0$, i.e. if and only if $\varphi(x)$ has a repeated root. 
We now move on to another space and define similar notions there. We begin by defining an equivalence relation on $\mathbb{A}^{n+1}$. We say that two points $p_{1}, p_{2} \in \mathbb{A}^{n+1}$ are equivalent if there exists a $\lambda \in K$ such that $p_{1}=\lambda p_{2}$. In this case we write $p_{1} \equiv p_{2}$. One may easily check that this is indeed an equivalence relation.

Definition 2.1.14 Using the above equivalence relation, let $\mathbb{P}^{n}$ be the set of non-zero equivalence classes on $\mathbb{A}^{n+1}$. We call $\mathbb{P}^{n}$ the $n$-dimensional projective space. We call the equivalence class of $p=\left(p_{1}, \ldots, p_{n+1}\right) \in \mathbb{A}^{n+1}$ a projective point or just as a point when the space is clear. We write $\left(p_{1}: \cdots: p_{n+1}\right)$ for a projective point. We call $\mathbb{P}^{1}$ the projective line and $\mathbb{P}^{2}$ the projective plane.

The projective line may be visualized as the set of slopes in the affine plane.

Example 2.1.15 Let $\left(p_{1}: p_{2}: p_{3}\right)$ be a projective point on the plane $\mathbb{P}^{2}$. We know by definition that one of $p_{1}, p_{2}$ or $p_{3}$ is nonzero. So without loss of generality, assume that $p_{3}$ is non-zero. Then $\left(p_{1}, p_{2}, p_{3}\right) \equiv\left(p_{1} / p_{3}, p_{2} / p_{3}, 1\right)$. Notice that we may always find a representative of an equivalence class with a 1 in at least one entry.

Definition 2.1.16 ([16] p.16) A closed projective subset $A \subseteq \mathbb{P}^{n}$ is a set consisting of all the common zeros of a set of homogeneous polynomial functions with coefficients in $K$. We will often refer to this a closed set for brevity.

Notice that a closed projective set is defined by homogeneous polynomials, i.e. a polynomial whose monomials are of the same degree. This ensures that it is well defined on the equivalence classes of $\mathbb{P}^{n}$. Indeed, let $A$ be a closed projective set defined by a homogeneous polynomial $F\left(T_{1}: \cdots: T_{n+1}\right)$ of degree $m$. Suppose that $F(p)=0$. Then $F(\lambda p)=\lambda^{m} F(p)=0$ as suggested. Throughout this work, we will let $T$ represent the set of variables $T_{1}, \ldots, T_{n+1}$. In keeping with this we will let $F(T)$ represent a polynomial function $F$ in the $n+1$ variables $T_{1}, \ldots, T_{n+1}$. When specifically discussing the projective plane we will use the variables $X, Y$, and $Z$.

The definitions of open, reducible, and irreducible projective sets follows exactly as in the affine definitions. We therefore omit rewriting these definitions. We now move on to discuss projective plane curves. 
Definition 2.1.17 Let $C$ be a closed projective subset of $\mathbb{P}^{2}$. If $C$ is defined by one non-constant homogeneous polynomial $F(X: Y: Z)$ then $C$ is called a projective plane curve.

Definition 2.1.18 ([16] p.12) Let $C$ be a projective plane curve defined by a homogeneous polynomial $F(X, Y, Z)$. A point $p \in C$ is called singular or is a singularity if $\frac{\partial F}{\partial X}(p)=\frac{\partial F}{\partial Y}(p)=\frac{\partial F}{\partial Z}(p)=0$. A point $p$ that is not singular is called nonsingular and a curve whose points are all nonsingular is called a nonsingular curve.

Example 2.1.19 Let $C$ be a projective plane curve defined by

$$
F(X: Y: Z)=Z^{n-2} Y^{2}-X^{n}+X Z^{n-1}
$$

for some $n>3$. Taking the partial derivatives, we have that

$\frac{\partial F}{\partial X}=-n X^{n-1}+Z^{n-1} \quad \frac{\partial F}{\partial Y}=2 Y Z^{n-2} \quad \frac{\partial F}{\partial Z}=(n-2) Z^{n-3} Y^{2}+(n-1) X Z^{n-2}$.

Since

$$
\frac{\partial F}{\partial X}(0: 1: 0)=0 \quad \frac{\partial F}{\partial Y}(0: 1: 0)=0 \quad \frac{\partial F}{\partial Z}(0: 1: 0)=0
$$

and the point $(0: 1: 0)$ is on $C$ it is singular. However if we consider the case where $n=3$ we see that

$$
\frac{\partial F}{\partial X}=-3 X^{2}+Z^{2} \quad \frac{\partial F}{\partial Y}=2 Y Z \quad \frac{\partial F}{\partial Z}=Y^{2}+2 X Z .
$$

Since

$$
\frac{\partial F}{\partial X}(0: 1: 0)=0 \quad \frac{\partial F}{\partial Y}(0: 1: 0)=0 \quad \frac{\partial F}{\partial Z}(0: 1: 0)=1
$$

the point $(0: 1: 0)$ is nonsingular. Upon further inspection, one can show that in the case $n=3$ all the points of $C$ are nonsingular, making $C$ a nonsingular curve.

For a general closed set we have the following definition of a singular point. 
Definition 2.1.20 ([16] p.12) Let $A \subset \mathbb{P}^{n}$ be a projective closed set defined by the homogeneous polynomials $F_{j}(T)$ for $j=1, \ldots, m$. A point $p \in A$ is called singular or is a singularity if $\frac{\partial F_{j}}{\partial T_{i}}(p)=0$ for all $j=1, \ldots, m, i=1, \ldots n$.

Looking back at the projective and affine planes, there is a strong relationship between the two. Consider the affine plane $\mathbb{A}^{2}$. We may embed this into $\mathbb{P}^{2}$ in a very natural way; we simply map $(x, y) \rightarrow(x: y: 1)$. Similarly we may also map $(x, y) \rightarrow(x: 1: y)$ or $(x, y) \rightarrow(1: x: y)$. Performing the inverses of these maps provides us with an affine cover of the projective plane. Indeed, for each point $p=\left(p_{1}: p_{2}: p_{3}\right) \in \mathbb{P}^{n}$ at least one of $p_{1}, p_{2}$, or $p_{3}$ is non-zero. Without loss of generality suppose $p_{3} \neq 0$. Then $p \equiv\left(p_{1} / p_{3}: p_{2} / p_{3}: 1\right)$ which is the image of $\left(p_{1} / p_{3}, p_{2} / p_{3}\right)$ under our first map. Hence, we may cover the projective plane with three copies of the affine plane. We give a name to the projective images of these maps.

Definition 2.1.21 Let $A \subset \mathbb{P}^{n}$ be a projective closed set. Let $L_{i} \subset \mathbb{P}^{n}$ be the projective closed set defined by the equation $T_{i}=0$. Then the open subsets $U_{i}=A \backslash L_{i}$ are called the affine pieces of $A$.

When examining properties of points we often examine neighbourhoods of that point. It will often be convenient to consider a neighbourhood equal to an affine piece. We will make this idea more explicit in later on.

\subsubsection{Homogenization and Dehomogenization}

There are strong relationships between affine and projective sets. We have already seen an example of this in our discussion about the affine pieces of a closed projective set. In this subsection we will make this relationship more explicit by introducing maps between affine and projective sets. We begin by defining a map from affine to projective sets. 
Definition 2.1.22 Let $A \subseteq \mathbb{A}^{n}$ be a closed affine set. We define the map $\Theta_{n+1}: A \rightarrow$ $\mathbb{P}^{n}$ by

$$
\left(t_{1}, \ldots, t_{n}\right) \rightarrow\left(t_{1}: \ldots: t_{n}: 1\right) \quad \forall\left(t_{1}, \ldots, t_{n}\right) \in A
$$

We call this map the homogenization of $A$ in the $(n+1)$ st variable. Clearly, we may define the homogenization $A$ in any variable by simply placing the 1 in that projective coordinate.

The image of the homogenization of $A$ is not often a closed projective set. However, there does exist a closed projective set which contains the image of the homogenization map.

Definition 2.1.23 Let $A \subseteq \mathbb{A}^{n}$ be a closed affine set defined by the polynomial $g\left(t_{1}, \ldots, t_{n}\right)$ of degree $m$. We define the homogenization of $g$ in the $n+1$ st variable as the homogeneous polynomial $G(T)$ where

$$
G(T)=T_{n+1}^{m} \cdot g\left(\frac{T_{1}}{T_{n+1}}, \ldots, \frac{T_{n}}{T_{n+1}}\right) .
$$

The closed projective set $S \subset \mathbb{P}^{n}$ defined by $G(T)$ is called the projective closure of $A$ in the $n+1$ st variable. For a closed set defined by several equations, we simply homogenize all defining polynomials and those will define a projective closure.

Example 2.1.24 Let $C$ be an affine plane curve defined by the polynomial $g(x, y)=$ $y^{2}-x^{3}+x$. We now find the homogenization of $c(x, y)$ in the third coordinate.

$$
\begin{aligned}
& G(X: Y: Z)=Z^{3} \cdot c\left(\frac{X}{Z}, \frac{Y}{Z}\right) \\
& G(X: Y: Z)=Z^{3}\left(\frac{Y^{2}}{Z^{2}}-\frac{X^{3}}{Z^{3}}+\frac{X}{Z}\right) \\
& G(X: Y: Z)=Y^{2} Z-X^{3}+X Z^{2} .
\end{aligned}
$$

Let $S_{3}$ be the closed projective set defined by $G(X, Y, Z)$. We see that the points of $C$ are in one-to-one correspondence to the points of $S_{3}$ of the form $(x: y: 1)$. Thus 
the only additional points on $S_{3}$ are of the form $(x: y: 0)$. However substitution into $G(X: Y: Z)$ we see that

$$
0=G(x: y: 0)=0-x^{3}-0=x^{3} .
$$

Thus there is only one additional point $p_{\infty}=(0: 1: 0)$ which is called the point at infinity. In general, the new points added when taking the projective closure are called infinite points.

Now, given a projective closed set, we look at mapping it to an affine one.

Definition 2.1.25 Let $A \subseteq \mathbb{P}^{n}$ be a closed projective set. We define the map $A \cap$ $U_{n+1} \rightarrow \mathbb{A}^{n}$ by

$$
\left(p_{1}: \cdots: p_{n+1}\right) \rightarrow\left(p_{1} / p_{n+1}, \ldots, p_{n} / p_{n+1}\right) \text { for all }\left(p_{1}: \cdots: p_{n+1}\right) \in A \cap U_{n+1}
$$

We call this map the dehomogenization of $A$ in the $(n+1)$ st variable. Clearly, we may define a dehomogenization of $A$ in any variable by simply dividing in that projective coordinate.

The image of the dehomogenization of $A$ is a closed affine set; it is defined by the polynomial $g(t)$ of the following definition.

Definition 2.1.26 Let $A \subseteq \mathbb{P}^{n}$ be a closed affine set defined by the homogeneous polynomial $G\left(T_{1}, \ldots, T_{n+1}\right)$ of degree $m$. We define the dehomogenization of $G$ in the $n+1$ st variable as the polynomial $g(t)$ where

$$
G(T)=T_{n+1}^{m} \cdot g\left(\frac{T_{1}}{T_{n+1}}, \ldots, \frac{T_{n}}{T_{n+1}}\right) .
$$

Making the change of coordinates $t_{i}=T_{i} / T_{n+1}$ for $i=1, \ldots, n$ we can express $g$ in affine coordinates. This is clearly a well-defined map at all points $p \in A$ with $p_{n+1} \neq 0$, which we have already defined as the affine piece $U_{n+1}$ of $A$. We may similarly define a dehomogenization in any projective variable. 
Definition 2.1.27 Let $A \subset \mathbb{A}^{n}$ be an affine set. Let $B$ be a projective open set. We say that the affine set $A$ is isomorphic to $B$ if there exists a dehomogenization of $B$, that is defined at all points $p \in B$, and whose image is $A$.

Example 2.1.28 Let $C$ be an projective plane curve defined by the polynomial

$$
G(X: Y: Z)=Y^{2} Z-X^{3}+X Z^{2}
$$

We now find the affine pieces of $C$.

$$
\begin{aligned}
G(X: Y: Z) & =Z^{3}\left(\frac{Y^{2}}{Z^{2}}-\frac{X^{3}}{Z^{3}}+\frac{X}{Z}\right)=Z^{3}\left(\left(\frac{Y}{Z}\right)^{2}-\left(\frac{X}{Z}\right)^{3}+\frac{X}{Z}\right) \\
& \Rightarrow g_{3}(x, y)=y^{2}-x^{3}+x, \\
G(X: Y: Z) & =Y^{3}\left(\frac{Z}{Y}-\frac{X^{3}}{Y^{3}}+\frac{X Z^{2}}{Y^{3}}\right)=Y^{3}\left(\frac{Z}{Y}-\left(\frac{X}{Y}\right)^{3}+\left(\frac{X}{Y}\right)\left(\frac{Z}{Y}\right)^{2}\right) \\
& \Rightarrow g_{2}(x, z)=z-x^{3}+x z^{2}, \\
G(X: Y: Z) & =X^{3}\left(\frac{Z Y^{2}}{X^{3}}-1+\frac{Z^{2}}{X^{2}}\right)=X^{3}\left(\left(\frac{Z}{X}\right)\left(\frac{Y}{X}\right)^{2}-1+\left(\frac{Z}{X}\right)^{2}\right) \\
& \Rightarrow g_{1}(y, z)=z y^{2}-1+z^{2} .
\end{aligned}
$$

The affine pieces of $C$ are isomorphic to the closed affine sets $U_{1}, U_{2}$ and $U_{3}$ defined by $g_{1}, g_{2}, g_{3}$ respectively. However, it may not me the case that the affine pieces are isomorphic to eachother. Although, we will see later on when we discuss rational maps that they are birationally equivalent.

\subsubsection{Regular Functions}

In this section we explore functions that are defined at all points of a set, namely regular functions. Regular functions will allow us to define what is meant by an isomorphism between curves. All properties discussed there after will be invariant under isomorphism. We begin with the definition of a regular function on an affine closed set. 
Definition 2.1.29 ([16] p.24) A function $g$ defined on a closed affine set $A \subseteq \mathbb{A}^{n}$ with values in $K$ is called regular if there exists a polynomial $G(t)$ with coefficients in $K$ such that $g(p)=G(p)$ for all $p \in A$.

The set of all regular functions on a closed set $A$ forms a ring under the usual operations ([16] p.25) and we give it a special name.

Definition 2.1.30 ([16] p.25) The ring of regular functions on a closed $A$ is called the coordinate ring of $A$ and it is denoted by $K[A]$.

We may clearly associate any polynomial $G(t) \in K[t]$ with a function in $g \in$ $K[A]$ by viewing it as a function on the set of points of $A$. This gives a natural homomorphism from $K[t] \rightarrow K[A]$. The kernel of this homomorphism is the set of polynomials $g(t) \in K[t]$ such that $g(p)=0$ for all $p \in A$ (in this case we often write $g(A)=0$ ). This is an ideal of $K[t]$, as it is the kernel of a homomorphism, and it gives rise to the following definition.

Definition 2.1.31 ([16] p.25) Let $I_{A}$ be the ideal of $K[t]$ consisting of all functions that are identically 0 on $A$. i.e $I_{A}=\{g(t) \in K[t] \mid g(p)=0, \forall p \in A\}$. This ideal is called the ideal of $A$.

By the comments above and using the First Isomorphism Theorem of rings, we see that $K[A]=K[t] / I_{A}$. We will spend the majority of this section examining the properties of the coordinate ring $K[A]$ and how it relates to properties of $A$. We will also be examining the structure of $I_{A}$. To that end, we state the following theorem without proof.

Theorem 2.1.32 ([4] p.13) (Hilbert's Basis Theorem) The ring $K[t]$ is Noetherian, i.e. all the ideals of $K[t]$ are finitely generated.

For a proof of this result see [4].

Let $A$ be a closed affine set and let $I_{A}=\left(F_{1}(t), \ldots, F_{m}(t)\right)$. Then it may be shown that the set of polynomials $F_{1}(t), \ldots, F_{m}(t)$ also define $A([4]$ p.11). This is why we 
may assume that a closed affine set is defined by a finite set of polynomials; Hilbert's Basis Theorem guarantees the existence of such a set.

We may now discuss the irreducibility of a closed set in terms of its ideal and defining equations.

Proposition 2.1.33 ([16] p.35) Let $C$ be an affine closed set. Then $C$ is irreducible if and only if $I_{C}$ is a prime ideal of $K[t]$.

Proof. Suppose that there exist closed sets $C_{1}$ and $C_{2}$ such that $C=C_{1} \cup C_{2}$ and $C_{1}, C_{2} \subsetneq C$. This means that exists a polynomial $c_{1}(t)$ that is zero on $C_{1}$, but not on $C$ and that there exists a polynomial $c_{2}(t)$ that is zero on $C_{2}$, but not on $C$. This is the case if and only if there exist functions $c_{\mathbf{1}}(t), c_{2}(t) \in K[C]$ such that $c_{1}(t), c_{2}(t) \neq 0$ but $c_{1}(t) c_{2}(t)=0$. Equivalently, $K[C]=K[t] / I_{C}$ is not an integral domain or $I_{C}$ is not prime.

Proposition 2.1.34 Let $C$ be an affine plane curve defined by an irreducible polynomial $c(x, y)$. If $C$ contains infinitely many points then $C$ is irreducible. Moreover, $I_{C}=(c(x, y))$.

Proof. Since $K[x, y]$ is a Unique Factorization Domain (see [2] p.306), irreducible elements generate prime ideals. Hence, showing $I_{C}=(c(x, y))$ gives us the irreducibility of $C$ by Proposition 2.1.33. Clearly $(c(x, y)) \subseteq I_{C}$. Now, suppose that $g(x, y) \in I_{C}$. Then $g(p)=0$ for all $p \in C$. Since $c(x, y)$ and $g(x, y)$ have infinitely many common zeros and since $c(x, y)$ is irreducible, it must be the case that $c(x, y)$ divides $g(x, y)$ by Proposition 2.1.7. This implies that $g(x, y) \in(c(x, y))$. Consequently $I_{C}=(c(x, y))$.

Example 2.1.35 Let $C$ be an affine plane curve defined by the polynomial $y^{2}-\varphi(x)$. If $\varphi(x)$ is not a square then $y^{2}-\varphi(x)$ is irreducible and hence so is $C$ by Proposition 2.1.34. In Example 2.1.13, we have shown that $C$ is singular if and only if $\varphi(x)$ has repeated roots. Thus, if $C$ is nonsingular then $C$ is irreducible. By Proposition 2.1.34 we know that $K[C] \cong K[x, y] /\left(y^{2}-\varphi(x)\right)$. 
Remark 2.1.36 From now on when we write $y^{2}-\varphi(x)$, it will be implied that $\varphi(x)$ has no repeated roots. This is allows us to assume that the affine curve defined by $y^{2}-\varphi(x)$ is irreducible and nonsingular.

Moving on to the projective setting, we obtain a slightly different definition of a regular function.

Definition 2.1.37 ([16] p.46) A function $g$ defined on a projective set $A \subseteq \mathbb{P}^{n}$ with values in $K$ is called regular if for all $p \in A$ there exist homogeneous polynomials $G(T), H(T)$ of the same degree with coefficients in $K$ such that $g(p)=G(p) / H(p)$ and $H(p) \neq 0$.

Unlike the definition of regular affine functions (Definition 2.1.29), regular projective functions are quotients of polynomials. The above definition is required to guarantee that regular projective functions are well-defined on projective points. Indeed, if we let $m$ represent the degree of $H(T)$ and $G(T)$, then

$$
g(\lambda p)=\frac{G(\lambda p)}{H(\lambda p)}=\frac{\lambda^{m} G(p)}{\lambda^{m} H(p)}=\frac{G(p)}{H(p)}=g(p) .
$$

As we will see in Subsection 2.1.6, the only regular functions of an irreducible projective closed set are constant functions. However, we need to some more tools to prove this result.

Remark 2.1.38 The projective definition of regular is just a generalization of the affine case. Indeed, assume that there exists a function $g(T)$ that is regular on a projective set $B$ isomorphic to an affine closed set $A$. Then at each point $p \in A$ we can express $g$ as $g(t)=\frac{G_{p}(t)}{H_{p}(t)}$ where $G_{p}(t), H_{p}(t) \in K[A]$ with $H_{p}(p) \neq 0$. Consider the ideal $I$ generated by all such functions $H_{p}$. By Hilbert's Basis Theorem (Theorem 2.1.32), $I$ is finitely generated, i.e. there exists a finite set of points $p_{1}, \ldots p_{k}$ such that $I=\left(H_{p_{1}}, \ldots, H_{p_{k}}\right)$. We now show that these functions do not have a common zero and are hence there greatest common divisor is 1 . Indeed, if the functions $H_{p_{1}}, \ldots, H_{p_{k}}$ had a common zero, at $p^{\prime}$ say, then so would all of the functions in $I$. 
However, $H_{p^{\prime}} \in I$ and is nonzero at $p^{\prime}$. Therefore, $H_{p_{1}}, \ldots, H_{p_{k}}$ are independent, and thus, by the Extended Euclidean Algorithm, there exists elements $u_{1}, \ldots, u_{k} \in K[A]$ such that

$$
\sum_{i=1}^{k} u_{i} H_{p_{i}}=1
$$

Now, multiplying by $g$ we obtain that

$$
\begin{aligned}
& g=\sum_{i=1}^{k} u_{i} H_{p_{i}} g \\
& g=\sum_{i=1}^{k} u_{i} G_{p_{i}}
\end{aligned}
$$

which is in $K[A]$.

\subsubsection{Regular Maps}

There are two definitions of regular maps; one for affine sets and one for projective sets. We begin by discussing the affine case.

Definition 2.1.39 ([16] p.27) Let $A \subseteq \mathbb{A}^{n}$ and $B \subseteq \mathbb{A}^{m}$ be closed sets. A map $g: A \rightarrow B$ is called a regular map if there exist $m$ regular functions $g_{1}, \ldots, g_{m}$ on $A$ with values in $K$ such that $g(p)=\left(g_{1}(p), \ldots, g_{m}(p)\right)$ for all $p \in A$.

Example 2.1.40 Let $C$ be an affine plane curve. The projection map $(x, y) \rightarrow x$ is a regular map from $C$ to $\mathbb{A}^{1}$.

Definition 2.1.41 ([16] p.29) Let $A \subseteq \mathbb{A}^{n}$ and $B \subseteq \mathbb{A}^{m}$ be closed sets. A regular map $g: A \rightarrow B$ is called an isomorphism if it has an inverse, i.e if there exists a regular function $h: B \rightarrow A$ such that $g \circ h=1_{B}$ and $h \circ g=1_{A}$. In this case, we say that $B$ and $A$ are isomorphic.

Example 2.1.42 Invertible linear transformations and translations are both isomorphisms of $\mathbb{A}^{n}$. 
In the projective setting we have the following definition of a regular map.

Definition 2.1.43 ([16] p.47) Let $A \subseteq \mathbb{P}^{n}$ and $B \subseteq \mathbb{P}^{m}$ be sets. A map $g: A \rightarrow B$ is called a regular map if there exist $m+1$ homogeneous polynomials $G_{1}, \ldots, G_{m}$ of the same degree on $A$ such that $G(p)=\left(G_{1}(p), \ldots, G_{m+1}(p)\right) \neq 0$ for all $p \in A$. As in the affine case, if a regular map has a regular inverse then we call it an isomorphism.

Due to the equivalence classes defining projective points we have an equivalence on regular projective maps.

Definition 2.1.44 Let $A \subseteq \mathbb{P}^{n}$ and $B \subseteq \mathbb{P}^{m}$ be sets. Let $g: A \rightarrow B$ be a regular map given by the $m+1$ homogeneous polynomials $G_{1}, \ldots, G_{m+1}$ of the same degree such that $G(p)=\left(G_{1}(p), \ldots, G_{m+1}(p)\right) \neq 0$ for all $p \in A$. Similarly, let $h: A \rightarrow B$ be a regular map given by the $m+1$ homogeneous polynomials $H_{1}, \ldots, H_{m+1}$ of the same degree such that $H(p)=\left(H_{1}(p), \ldots, H_{m+1}(p)\right) \neq 0$ for all $p \in A$. Then we say that $h$ is equivalent to $g$ if

$$
H_{i} G_{j}=G_{i} H_{j} \quad \text { for all1 } \leq i, j \leq m+1 .
$$

It will also be useful for us to know the following.

Proposition 2.1.45 ([16] p.57) The image of an irreducible projective closed set under a regular projective map is a closed set.

To prove this result would require exploring the graph of a projective function, a topic outside our scope. However we can prove the following.

Proposition 2.1.46 ([16] p.48) The preimage of a projective closed set under a regular projective map is a closed set.

Proof. Let $A$ be a closed projective set defined by the polynomials $a_{1}(T), \ldots, a_{k}(T)$. Let $g$ be a regular map $g: S \rightarrow A$. The preimage $S$ is the projective set defined by the common zeros of $a_{1}(g(T)), \ldots, a_{k}(g(T))$ and hence a closed set. 
Example 2.1.47 Continuing from Example 2.1.24, let $H(X: Y: Z)$ be the homogenization of $c(x, y)$ in the second variable and let $S_{2}$ be the projective closure of $C$ in the second variable. Then $H(X: Y: Z)=Z^{2} Y-X^{3}+X Y^{2}$ and we define the regular map $\Phi: \mathbb{P}^{2} \rightarrow \mathbb{P}^{2}$ by $\Phi(X: Y: Z)=(X: Z: Y)$. We see that $\Phi$ is simply a change of coordinates. Consequently, $\Phi\left(S_{2}\right)=S_{3}$ and $\Phi\left(S_{3}\right)=S_{2}$. Thus the projective closures are isomorphic. Generalizing this, we may see that projective closures in any variable are isomorphic.

As just seen in the previous example, a change of coordinates is an isomorphism. Thus, homogenizing a polynomial $g(t)$ in any variable, yields isomorphic closed projective sets. For this reason, we often just homogenize a polynomial $g(t)$ in the last variable, and (abusively) refer to this as the homogenization of $g(t)$. Similarly we (abusively) refer to the projective closure in the last variable as the projective closure. As such, we will denote the projective closure of an affine closed set $S$ as $\bar{S}$. Now, given a projective set, we return to our examination of its affine pieces.

Lemma 2.1.48 Let $A \subset \mathbb{P}^{n}$ be a closed projective set. Then the affine pieces $U_{i}$ of $A$ are isomorphic to closed affine sets for all $i=1, \ldots, n+1$.

Proof. Suppose that $A$ is defined by the homogeneous polynomials $a_{j}(T)$, for some $j=1, \ldots, m$. We recall that $U_{i}=\left\{p \in A \mid p_{i} \neq 0\right\}$. As such, all the points of $U_{i}$ may be written as $p=\left(p_{1}: \cdots: p_{i-1}: 1: p_{i+1}: \cdots: p_{n+1}\right)$ where $p \in A$. Thus the $U_{i}$ is the set of points such that $a_{j}\left(T_{1}: \cdots: T_{i-1}: 1: T_{i+1}: \cdots: T_{n+1}\right)=0$ for all $j=1, \ldots, m$. Now, identifying $t_{k}$ with $T_{k}$ for $k=1, \ldots, i-1$ and $t_{k}$ with $T_{k+1}$ for $k=i \ldots n$ gives the isomorphism $A_{i} \subset \mathbb{A}^{n} \leftrightarrow U_{i}$ where $A_{i}$ is defined by the equations $a_{j}\left(t_{1}, \ldots, t_{i-1}, t_{i+1}, \ldots, t_{n+1}\right)=0$ for $j=1, \ldots, m$.

Often, when examining projective points, it is helpful to consider neighbourhoods around that point. If we consider a projective curve $C$ then we know that every point on $C$ lies in some affine piece of $C$. So when considering a projective neighbourhood of a point, it would be helpful to assume that it behaves like an affine neighbourhood of the piece. Indeed, to each point such neighbourhoods exist. 
Proposition 2.1.49 Let $A \subset \mathbb{P}^{n}$ be a closed projective set. Any point on $A$ has a projective neighbourhood $U$ isomorphic to an affine neighbourhood.

Proof. Let $p=\left(p_{1}: \cdots: p_{n+1}\right) \in \mathbb{P}^{n}$ be a projective point. There exists a $p_{i}$ not equal to zero. Let $L_{i}$ be the closed projective set defined by the equation $T_{i}=0$. Then the affine piece $U_{i}$ is such that $U_{i}=A \backslash L_{i}$ and is thus an open projective subset of $A$. This is an affine piece and is thus isomorphic to an affine neighbourhood.

We may now prove the result we stated in the introduction; that everywhere regular functions on a closed projective set are constant.

Proposition 2.1.50 ([16] p.59) Let $A$ be an irreducible closed projective set. If $g$ is a regular function then it is constant.

Proof. We may view $g$ as a map $g: A \rightarrow \mathbb{A}^{1}$. Define $g^{\prime}: A \rightarrow \mathbb{P}^{1}$ by $g^{\prime}(p)=(g(p): 1)$. Then since $g$ is regular, by Definition 2.1.43, $g^{\prime}$ is a regular map. From Proposition 2.1.45 we know that the image of $g^{\prime}$ is a closed set. In addition, the image $g^{\prime}$ may be viewed as a subset of $\mathbb{A}^{1}$ by considering the affine piece $U_{i}$. In this light, the image of $g^{\prime}$ is isomorphic to the image of $g$, which defines an affine closed set. Therefore $g(A)=\mathbb{A}^{1}$ or $g(A)$ is a finite set of points (Example 2.1.7). The first case is impossible since the image of $g^{\prime}$ is supposed to be closed in $\mathbb{P}^{1}$ and $\mathbb{A}^{1}$ is not. Suppose $g(A)=\left\{p_{1}, \ldots, p_{k}\right\}$. Then this would mean that $A=\cup_{i=1}^{k} g^{-1}\left(p_{i}\right)$ and hence $A$ is the union of closed sets by Proposition 2.1.46. Thus, if the image of $G$ is more than one point, $A$ is reducible; a contradiction. The image of $g$ must therefore be a single point, i.e. $g$ is constant.

From the above Proposition, we see that being regular on an irreducible closed projective set is too strict a condition. This is why we are more interested in rational functions. We also note that Proposition 2.1.50 is about irreducible closed projective sets only. There do exist nonconstant regular functions on open projective sets. This will be made more explicit when we discuss rational functions. 


\subsubsection{Rational Functions}

Definition 2.1.51 ([16] p.35) Let $A$ be an irreducible closed affine set. The field of fractions of the coordinate ring $K[A]$ is called the rational function field of $A$. We denote this by $K(A)$, i.e.

$$
K(A)=\left\{\frac{g(t)}{h(t)} \mid g(t), h(t) \in K[A], h(A) \neq 0\right\} .
$$

Two rational functions $\frac{g(t)}{h(t)}, \frac{g^{\prime}(t)}{h^{\prime}(t)} \in K[A]$ are considered equal if $g(t) h^{\prime}(t)=$ $g^{\prime}(t) h(t)$. For this reason we may and shall always assume that the numerator and denominator of a rational function have no common factors.

Let $C$ be an irreducible plane affine curve defined by the polynomial $y^{2}-\varphi(x)$. By Proposition 2.1.34, the ring of regular functions $K[C]$ may be written as

$$
K[C]=\frac{K[x, y]}{\left(y^{2}-\varphi(x)\right)}
$$

The rational function field is the field of fractions of $K[C]$, and thus a field. Let $K(x)$ denote the rational function field in one variable $x$. Then we may view $K(x)$ as a subfield of $K(C)$, or, more importantly, $K(C)$ as an extension field of $K(x)$

$$
K(C)=\frac{K(x)[y]}{\left(y^{2}-\varphi(x)\right)}=K(x)(y) \text { where } y^{2}-\varphi(x)=0
$$

We use this identity in the following remark.

Remark 2.1.52 Let $C$ be an irreducible plane affine curve defined by the polynomial $y^{2}-\varphi(x)$. Since $K(x)(y)$ is a degree two extension of $K(x)$ we may express the elements $K(C)$ as

$$
K(C)=\left\{\frac{a(x)}{b(x)}+\frac{c(x)}{d(x)} y \mid a(x), b(x), c(x), d(x) \in K[x]\right\} .
$$

We will usually express the elements of $K(C)$ is this manner. 
We now move on to the projective setting.

Definition 2.1.53 ([16] p.50) Let $A$ be an irreducible closed projective set. Let $g(T), h(T) \in K[T]$ be homogeneous polynomials of the same degree. Let

$$
K(A)=\left\{\frac{g(T)}{h(T)} \mid h(A) \neq 0\right\}
$$

Under the usual operations $K(A)$ forms a field. We call $K(A)$ the rational function field of $A$.

The next few definitions are true for both the affine and projective setting (adapting respective definitions as needed). We will use $T^{\prime}$ to represent an arbitrary variable space.

Definition 2.1.54 ([16] p.36) Let $A$ be an irreducible closed set (affine or projective). Let $\frac{g\left(T^{\prime}\right)}{h\left(T^{\prime}\right)} \in K(A)$. Then the set of points at which $h\left(T^{\prime}\right)=0$ is a closed subset $S$ of $A$. The open set $D=A \backslash S$ is called the domain of definition and it is the set of points at which $\frac{g\left(T^{\prime}\right)}{h\left(T^{\prime}\right)}$ is defined. We say that the rational function is regular at these points.

Proposition 2.1.55 ([16] p.37) Let $A$ be an irreducible closed set (affine or projective). Then a rational function $\phi \in K(A)$ is uniquely determined if it is specified on some non-empty open subset $U \subset D \subset A$, where $D$ is the domain of definition of $\phi$

Proof. Suppose $\phi(p)=0$ for all $p \in U$ and $\phi(A) \neq 0$. Then we may represent $A$ as the union of two closed sets $D_{1}$ and $D_{2}$, contradicting the irreducibility of $A$. Indeed, take any expression $\phi(T)=\frac{g(T)}{h(T)}$. We then define $D_{1}$ by the set $g(T)=0$ and define $D_{2}=D \backslash U$. Then $A=D_{1} \cup D_{2}$, with $D_{1}, D_{2} \subsetneq A$, which contradicts the irreducibility of $A$. Thus $\phi(A)=0$ on all of $A$.

Given two rational functions, $\phi_{1}$ and $\phi_{2}$ that are equal on some open set $U \subset D$, their difference $\phi_{1}-\phi_{2}$ is zero on $U$. From the above, this implies that $\phi_{1}-\phi_{2}=0$ on $A$ and thus $\phi_{1}=\phi_{2}$. 
Corollary 2.1.56 Let $A$ be an irreducible closed projective set. A rational function $\phi$ is zero on a non-empty open subset of $A$ if and only if it is zero on $A$.

Using the above results we may also define a rational function as follows.

Corollary 2.1.57 A regular function on an open subset $U \subset A$ (affine or projective) is a rational function on $A$.

It is sometimes convenient to view regular functions on open sets in this way.

Remark 2.1.58 Note that rewording Remark 2.1.38 shows that an affine rational function that is regular on a closed affine set, is a regular affine function as defined in Definition 2.1.29. This identification is also useful.

\subsubsection{Rational Maps}

As we did with regular functions in Subsection 2.1.4, we may construct maps using rational functions. As usual, we begin with the affine case.

Definition 2.1.59 ([16] p.37) Let $A \subset \mathbb{A}^{m}$ and $B \subset \mathbb{A}^{n}$ be closed sets. A rational map $\Phi: A \rightarrow B$ is an $m$-tuple of rational functions $\Phi_{1}, \ldots, \Phi_{m} \in K(A)$ such that, for all points $p \in A$ at which all the $\Phi_{i} i=1, \ldots, m$ are regular, we have that $\Phi(p)=\left(\Phi_{1}(p), \ldots, \Phi_{m}(p)\right) \in B$. We say that $\Phi$ is regular or defined at such a point $p$.

Before we consider birational maps between closed sets we need the following definition.

Definition 2.1.60 Let $A$ be a closed set. A subset $S \subset A$ is dense in $A$ if for every open subset $U$ of $A, U \cap S \neq \emptyset$.

Definition 2.1.61 ([16] p.38) Let $A$ and $B$ be affine closed sets. A rational map $\Phi$ : $A \rightarrow B$ is called a birational equivalence if $\Phi$ has an inverse rational map $\Psi: B \rightarrow A$, that is if $\Phi(A)$ is dense in $B$ and $\Psi(B)$ is dense in $A$, and $\Phi \circ \Psi=1_{B}, \Psi \circ \Phi=1_{A}$ 
(where defined) where $1_{B}$ and $1_{A}$ are the identity maps on $B$ and $A$ respectively. In this case we say that $A$ and $B$ are birationally equivalent.

Example 2.1.62 Let $C$ be an affine plane curve defined by the polynomial $y^{2}-\varphi(x)$. If the degree of $\varphi(x)$ is even, $2 m$ say, then we may find a curve birationally equivalent to $C$ where the degree is odd. Since $K$ is algebraically closed we may rewrite $\varphi(x)=$ $q(x)(x-a)$ for some $a \in K$. Now dividing $y^{2}-\varphi(x)$ by $(x-a)^{2 m}$ we obtain,

$$
0=\left(\frac{y}{(x-a)^{m}}\right)^{2}-\frac{q(x)}{(x-a)^{2 m-1}}
$$

Rewriting $q(x)$ as a polynomial in $x-a$, say $q(x)=h(x-a)$, then

$$
\begin{aligned}
0 & =\left(\frac{y}{(x-a)^{m}}\right)^{2}-\frac{q(x)}{(x-a)^{2 m-1}} \\
& =\left(\frac{y}{(x-a)^{m}}\right)^{2}-\frac{h(x-a)}{(x-a)^{2 m-1}} \\
& =\left(\frac{y}{(x-a)^{m}}\right)^{2}-f\left(\frac{1}{x-a}\right)
\end{aligned}
$$

where $f$ is a polynomial. This shows that $C$ is birational to the curve $v^{2}-f(\xi)$ where $v=\frac{y}{(x-a)^{m}}$ and $\xi=\frac{1}{x-a}$. Here, $\operatorname{deg}(f(\xi)) \leq 2 m-1$, and could possibly be even. If so, then we may write $f(\xi)=q_{2}(\xi)\left(\xi-a_{2}\right)$ and repeat the above process. After each repetition, the degree of $f(x)$ remains positive, but decreases. So this process either stops when the degree of $f(x)$ is odd or zero. If it stops when the degree of $f$ is zero then $C$ is birationally equivalent to a curve defined by $(y-a)(y+a)$ for some $a \in K$. Since this curve is reducible and $C$ is not, we have a contradiction. Therefore $C$ is birational to a curve of the form $v^{2}-f(\xi)$ where $\operatorname{deg}(f)$ is odd.

Remark 2.1.63 In view of Example 2.1.62, when we talk about an affine plane curve $C$ defined by the polynomial $y^{2}-\varphi(x)$, we will not only assume that $C$ is irreducible and non-singular, we will now also assume that the degree of $\varphi(x)$ is odd.

For a point on an arbitrary curve we may rationally map it to a nonsingular point. 
Theorem 2.1.64 Let $C$ be an affine plane curve defined by the polynomial $c(x, y)$. Let $p \in C$ be a singular point. Then there exists an affine plane curve birationally equivalent to $C$ where the image of $p$ is nonsingular.

Proof. As translations are isomorphisms (Defintion 2.1.43) we may assume without loss of generality that $p=(0,0)$. We may express $c(x, y)$ as

$$
c(x, y)=c_{m}(x, y)+c_{m+1}(x, y)+\cdots+c_{n}(x, y)
$$

for some homogeneous polynomials $c_{i}(x, y)$ of degree $i$. Since $p=(0,0)$ is singular, we will see that $m>1$ in Example 2.2.7. Consider $c_{m}(x, y)$, it may be written as

$$
c_{m}(x, y)=\sum_{i=0}^{m} a_{i} x^{i} y^{m-i}
$$

for some $a_{i} \in K$. We may rearrange terms to obtain that

$$
c_{m}(x, y)=\sum_{i=0}^{m} a_{i}\left(\frac{x}{y}\right)^{i} y^{m}
$$

As $K$ is algebraically closed, there exists a root $k$ of the equation $\sum_{i=0}^{m} a_{i} X^{i}=0$. So by applying the regular map $x \mapsto x+k y$, which fixes $(0,0)$, we obtain that

$$
c_{m}(x, y) \mapsto \sum_{i=0}^{m} a_{i}\left(\frac{x+k y}{y}\right)^{i} y^{m}=\sum_{i=0}^{m} a_{i}\left(\frac{x}{y}+k\right)^{i} y^{m}
$$

However, expanding this expression we have that the coefficient of $y^{m}$ is

$$
\sum_{i=0}^{m} a_{i} k^{i}=0
$$

So without loss of generality we may assume that

$$
c_{m}(x, y)=\sum_{i=1}^{m} a_{i} x^{i} y^{m-i}
$$


We now return to the polynomial $c(x, y)$. As above, we may write it as

$$
\begin{aligned}
c(x, y) & =c_{m}(x, y)+c_{m+1}(x, y)+\cdots+c_{n}(x, y) \\
& =\sum_{i=1}^{m} a_{m_{i}} x^{i} y^{m-i}+\sum_{i=0}^{m+1} a_{(m+1)_{i}} x^{i} y^{m+1-i}+\cdots+\sum_{i=0}^{n} a_{n_{i}} x^{i} y^{n-i} \\
& =y^{m}\left(\sum_{i=1}^{m} a_{m_{i}}\left(\frac{x}{y}\right)^{i}+y \sum_{i=0}^{m+1} a_{(m+1)_{i}}\left(\frac{x}{y}\right)^{i}+\cdots+y^{n-m} \sum_{i=0}^{n} a_{n_{i}}\left(\frac{x}{y}\right)^{i}\right) .
\end{aligned}
$$

So making the change of coordinates $x / y=z, C$ is birationally equivalent to the affine plane curve $D$ defined by the equation,

$$
d(z, y)=\sum_{i=1}^{m} a_{m_{i}} z^{i}+y \sum_{i=0}^{m+1} a_{m+1_{i}} z^{i}+\cdots+y^{n-m} \sum_{i=0}^{n} a_{n_{i}} z^{i}
$$

The point $(0,0)$ is indeed in $D$ as the first summation has no constant term by assumption. As $K$ is algebraically closed, each of the summations in $z$ factors into a product of linear factors, i.e.

$$
d(z, y)=\prod_{i=1}^{m}\left(z-b_{1_{i}}\right)+y \prod_{i=0}^{m+1}\left(z-b_{2_{i}}\right)+\cdots+y^{n-m} \prod_{i=0}^{n}\left(z-b_{n_{i}}\right) .
$$

Consider the first summation above. Since $(0,0) \in D$ at least one of $b_{1_{1}}, b_{1_{2}}, \ldots b_{1_{m}}$ is zero. By re-indexing if necessary, we may assume that $b_{1_{1}}=b_{1_{2}}=\cdots=b_{1_{l}}=0$. Now applying the birational map $y \mapsto y z^{l} \prod_{i=l+1}^{m}\left(z-b_{1_{i}}\right)$ we obtain that

$$
\begin{aligned}
d(x, y) \mapsto & z^{l} \prod_{i=l+1}^{m}\left(z-b_{1_{i}}\right)\left(z+y \prod_{i=0}^{m+1}\left(z-b_{2_{i}}\right)+\right. \\
& \left.\cdots+y^{n-m} z^{(n-m-1)(l-1)} \prod_{i=l}^{m}\left(z-b_{1_{i}}\right)^{n-m-1} \prod_{i=0}^{n}\left(z-b_{n_{i}}\right)\right) .
\end{aligned}
$$


Therefore $D$ is birationally equivalent to the curve $D^{\prime}$ defined by

$$
\begin{aligned}
d^{\prime}(z, y)= & z+y \prod_{i=0}^{m+1}\left(z-b_{2_{i}}\right)+\cdots \\
& +y^{n-m} z^{(n-m-1)(l-1)} \prod_{i=l}^{m}\left(z-b_{1_{i}}\right)^{n-m-1} \prod_{i=0}^{n}\left(z-b_{n_{i}}\right) \\
= & z+y \cdot g(z, y) .
\end{aligned}
$$

Lastly, we just need to check that $(0,0) \in D^{\prime}$ is non-singular. Checking $\frac{\partial d^{\prime}}{\partial z}(0,0)$ we see that

$$
\frac{\partial d^{\prime}}{\partial z}(0,0)=1+0 \cdot \frac{\partial g}{\partial z}(0,0)=1 .
$$

Therefore, by Definition $2.1 .12,(0,0)$ is nonsingular.

Note that the resulting curve may still have other singularities. What we have shown is that a point being singular is not invariant under birational equivalence. We now demonstrate something that is invariant under birational equivalence.

Proposition 2.1.65 ([16] p.12) Two affine closed sets $A$ and $B$ are birationally equivalent if and only if their function fields $K(A)$ and $K(B)$ are isomorphic.

Sketch of Proof. Let $A \subset \mathbb{A}^{n}=\left(t_{1}, \ldots, t_{n}\right)$ and $B \subset \mathbb{A}^{m}=\left(s_{1}, \ldots, s_{m}\right)$. Now $A$ and $B$ being birationally equivalent implies that there exist a birational map $\Phi=$ $\left(\Phi_{1}(t), \ldots, \Phi_{m}(t)\right)$ from $A$ to $B$. Thus given a rational function $r\left(s_{1}, \ldots, s_{m}\right) \in K(B)$, we may send it to $r\left(\left(\Phi_{1}(t), \ldots, \Phi_{m}(t)\right) \in K(A)\right.$. This defines an isomorphism $K(B) \rightarrow$ $K(A)$ of these two fields. Conversely, if $K(A)$ and $K(B)$ are isomorphic then under this isomorphism $t_{1}, \ldots, t_{n}$ correspond to rational functions $r_{1}(s), \ldots, r_{n}(s) \in K(B)$. Similarly, $s_{1}, \ldots, s_{m}$ correspond to rational functions $f_{1}(t), \ldots, f_{m}(t) \in K(A)$. Then the maps $\phi: B \rightarrow A$ defined by $\phi(s)=\left(r_{1}(s), \ldots, r_{n}(s)\right)$ and $\psi: A \rightarrow B$ defined by $\psi(t)=\left(f_{1}(t), \ldots, f_{m}(t)\right)$ are birational.

Moving onto the projective setting, we define rational maps between projective sets. 
Definition 2.1.66 ([16] p.37) Let $A \subset \mathbb{P}^{m}$ and $B \subset \mathbb{P}^{n}$ be closed sets. A rational map $\Phi: A \rightarrow B$ is an $m$-tuple of rational functions $\Phi_{1}, \ldots, \Phi_{m} \in K(A)$ such that, for all points $p \in A$ at which all the $\Phi_{i} i=1, \ldots, m$ are regular, we have that $\Phi(p)=\left(\Phi_{1}(p): \cdots: \Phi_{m+1}(p)\right) \in B$. We say that $\Phi$ is regular or defined at such a point $p$.

Just as in the case of regular functions, due to the equivalence classes defining projective points we have an equivalence on regular projective maps.

Definition 2.1.67 Let $A \subseteq \mathbb{P}^{n}$ and $B \subseteq \mathbb{P}^{m}$ be sets. Let $g: A \rightarrow B$ be a regular map given by the $m+1$ rational functions $G_{1}, \ldots, G_{m+1}$ such that $G(p)=$ $\left(G_{1}(p), \ldots, G_{m+1}(p)\right)$. Sinilarly, let $h: A \rightarrow B$ be a regular map given by the $m+1$ rational functions $H_{1}, \ldots, H_{m+1}$ such that $H(p)=\left(H_{1}(p), \ldots, H_{m+1}(p)\right)$. Then we say that $h$ is equivalent to $g$ if

$$
H_{i} G_{j}=G_{i} H_{j} \quad \text { for all } \leq i, j \leq m+1 .
$$

The definition of birationally equivalent projective sets is the same as the affine case. We now have the ability to define regular maps on arbitrary sets.

Definition 2.1.68 Let $A$ be a set (affine or projective). A regular map on an set $A$ is a rational map defined at every point $p \in A$. A regular map on an set with a regular inverse is again called an isomorphism.

As per Corollary 2.1.57 we may describe a birational maps as follows.

Lemma 2.1.69 ([16] p.52) Two closed sets $A$ and $B$ (affine or projective) are birationally equivalent if and only if contain isomorphic open sets $U \subset A$ and $V \subset B$.

Proof. One direction is clear. If $U$ and $V$ are isomorphic then the regular map from $U$ to $V$ defines a rational map for $A$ to $B$ as rational functions are uniquely determined when defined on open subsets (Proposition 2.1.55). 
To prove the converse, we prove the projective case as the proof of the affine case follows similarly. Let $\Phi$ be a birational map between the projective closed sets $A$ to $B$. Set $U_{1}$ as

$$
U_{1}=\{p \in A \mid \Phi(p) \text { is defined }\}
$$

Set $V_{1}$ as

$$
V_{1}=\left\{p \in A \mid \Phi^{-1}(p) \text { is defined }\right\} .
$$

By Definition 2.1.66, $\Phi\left(U_{1}\right)$ is dense in $B$ and thus $\Phi^{-1}\left(V_{1}\right) \cap U_{1}$ is open and nonempty. Set $U=\Phi^{-1}\left(V_{1}\right) \cap U_{1}$ and $V=\Phi\left(U_{1}\right) \cap V_{1}$. Then $\Phi(U)=V$ and $\Phi^{-1}(V)=U$ are both regular and hence define an isomorphism between $U$ and $V$.

Birational maps provide a means to define curves in higher dimensional space.

Definition 2.1.70 ([4] p.150) A projective (or affine) curve is a closed projective (or affine) set birationally equivalent to a plane projective (or affine) curve.

The remainder of our results about rational maps will come from considering the affine pieces and projective closures of curves.

Lemma 2.1.71 Let $C$ be an irreducible affine plane curve and let $\bar{C}$ be its projective closure. Then the function fields $K(C)$ and $K(\bar{C})$ are isomorphic.

Proof. From 2.1.49 we know that $C$ may be viewed as an open subset of $\bar{C}$. From Corollary 2.1.56 we also know that the rational functions that are zero on $C$ are exactly those that are zero on $\bar{C}$. Thus, as per the definition of the set of projective rational functions (Definition 2.1.53), $K(C) \cong K(\bar{C})$.

Corollary 2.1.72 Let $C$ be an irreducible plane projective curve, and let $C_{1}, C_{2}, C_{3}$ be its affine pieces. Then the function fields $K\left(C_{1}\right), K\left(C_{2}\right)$ and $K\left(C_{3}\right)$ are isomorphic.

Example 2.1.73 From Lemma 2.1.71, we know that an irreducible plane projective curve and its affine pieces have isomorphic function fields. In this example we demonstrate that isomorphism. Let $C$ be an irreducible plane affine curve defined 
by the polynomial $c(x, y)$. Let $\bar{C}$ be the projective closure of $C$. It is defined by the homogenization of $c(x, y)$ which we denote as $\bar{c}(X: Y: Z)$. The function field of $\bar{C}$ is

$$
K(\bar{C})=\left\{\frac{G(X: Y: Z)}{H(X: Y: Z)} \mid \operatorname{deg}(G)=\operatorname{deg}(H), H(\bar{C}) \neq 0\right\} .
$$

Consider the natural generalization of homogenization of a polynomial to that of a rational function. It defines a ring homomorphism of homogenization, $K(C) \rightarrow K(\bar{C})$. It is a nonzero ring homomorphism from a field and hence one-to-one ([2] p.254). To show that homogenization is also surjective, we notice for all homogeneous polynomials $H(X: Y: Z)$ there exists an $h(x, y)=H(x: y: 1)$ whose homogenization is $H(X: Y: Z)$. This construction extends naturally to rational functions. For any $h(x, y) \neq 0$ on $C$, its homogenization $H(X: Y: Z) \neq 0$ on $\bar{C}$ since is defined at all points $(x: y: 1)$. Homogenization is thus a bijective ring homomorphism between fields, i.e. defines a field isomorphism. Clearly, the natural extension of dehomogenization to rational functions $K(\bar{C}) \rightarrow K(C)$ defines its inverse.

When we are discussing rational functions on a plane projective curve $C$, we typically only look at the affine representation of the function field $K(C)=K(x, y)$ as described in Remark 2.1.52. Since the affine and projective representations are isomorphic, this abuse is justified. Secondly, from Corollary 2.1.72, we also know that all the affine pieces of a projective curve have isomorphic function fields, so it doesn't matter which piece we consider. Nevertheless, is may be the case that the affine pieces themselves may not be isomorphic. However, as we will presently see, they are related.

Corollary 2.1.74 Let $C$ be an irreducible projective plane curve. Then the affine pieces of $C$ are birationally equivalent.

Proof. By Corollary 2.1.72, we know that the affine pieces of $C$ have isomorphic function fields. Therefore, by Proposition 2.1.65, they are birationally equivalent. 
The birational maps are given by homogenizing an affine piece and dehomogenizing it in a different variable; both of which are birational maps.

We conclude this section by proving an important result.

Theorem 2.1.75 Two projective closed sets $A$ and $B$ are birationally equivalent if and only if their function fields $K(A)$ and $K(B)$ are isomorphic.

Proof. Two closed projective sets $A$ and $B$ are birationally equivalent then using Definition 2.1.69 of a rational map, there exists open subsets $V_{A} \subset A$ and $V_{B} \subset$ $B$ such that $V_{A}$ and $V_{B}$ are isomorphic. Restricting the isomorphism to the first affine pieces $U_{A} \subset A$ and $U_{B} \subset B$ gives an isomorphism from $U_{A} \cap V_{A}$ to $U_{A} \cap$ $V_{B}$. We know that affine pieces of $U_{A}$ and $U_{B}$ are respectively isomorphic to affine closed sets $S_{A}$ and $S_{B}$. Thus we have an isomorphism between open subsets of affine closed sets, i.e. by Definition 2.1.69, the affine closed sets $S_{A}$ and $S_{B}$ are birationally equivalent. So by Proposition 2.1.65, the function fields $K\left(S_{A}\right)$ and $K\left(S_{B}\right)$ are isomorphic. Furthermore, by Lemma 2.1.71, $K(A) \cong K\left(S_{A}\right)$ and $K(B) \cong$ $K\left(S_{B}\right)$. Consequently, $K(A) \cong K(B)$ as desired.

By Definition 2.1.70, a curve is birationally equivalent to a projective plane curve, so we have the following corollary.

Corollary 2.1.76 Let $C$ be an irreducible curve. Then the function field $K(C)$ is isomorphic to $K(x, y) /(c(x, y))$ for some irreducible polynomial $c(x, y)$.

\section{$2.2 \quad$ Tangent Lines}

Following the usual geometric definition of a tangent line, we will define tangent lines of closed sets. The equation of a tangent line will become useful in the following section. We will also associate a closed set to tangent lines called the tangent space. We simultaneously discover how the tangent space is dependent on whether or not the point of interest is singular. Clearly, we must first define what we mean by a line. 
Definition 2.2.1 An affine line $L \in \mathbb{A}^{n}$ is a closed affine set parameterized by $L=\{m t+p \mid t \in K\}$ where $m, p \in \mathbb{A}^{n}$.

We know from linear algebra that lines $L$ in the affine plane may equivalently be defined by polynomials of the form $l(x, y)=a x+b y+c$. We will often go back and forth between these expressions of $L$.

Definition 2.2.2 ([16] p.85) Let $A$ be an affine closed set defined by the polynomials $a_{1}(T), \ldots, a_{n}(T)$. Let $L$ be a line parameterized by $L=\{m t+p \mid t \in K\}$. The intersection multiplicity of the line $L$ with a closed $A$ at $p$ is the multiplicity of $t=0$ as a root of $g c d\left(a_{1}(m t+p), \ldots, a_{n}(m t+p)\right)$.

In the case that the affine closed set $A$ is defined by one polynomial, Definition 2.2.2 may be simplified.

Definition 2.2.3 ([16] p.85) Let $A$ be an affine closed set defined by the polynomial $a(T)$. Let $L$ be a line parameterized by $L=\{m t+p \mid t \in K\}$. The intersection multiplicity of the line $L$ with a closed $A$ at $p$ is the multiplicity of $t=0$ as a root of $a(m t+p)$.

Let $C$ be an affine plane curve defined by the polynomial $c(x, y)$. Let $L$ be a line defined by the polynomial $l(x, y)$ where $L$ is not identically 0 on $C$. We may then consider the the closed set $C \cap L$ defined by the set of equations $\{l(x, y)=0, c(x, y)=$ $0\}$. Let us begin by looking at a concrete example.

Example 2.2.4 For this example we let $K=\mathbb{C}$. Let $C$ be a curve defined by the polynomial $c(x, y)=y^{2}-x^{2}-x+1$. Let $L$ be the line defined by the polynomial $-3(x-1)+2(y-1)$. Parameterizing $L$, we may instead describe $L$ as the point set $(2,3) t+(1,1)$ where $t \in K$. This permits a simpler expression for the common zeros of $c(x, y)$ and $l(x, y)$ which define $C \cap L$. Indeed, by substitution, $C \cap L$ is defined by the equation

$$
c(2 t+1,3 t+1)=(3 t+1)^{2}-(2 t+1)^{2}-(2 t+1)+1=5 t^{2} .
$$


Thus $C \cap L$ consists only of the point corresponding to $t=0$; namely $p=(1,1)$.

Definition 2.2.5 ([16] p.85) Let $A$ be an affine closed set. A line $L$ is tangent to a closed set $A$ at $p$ if it has intersection multiplicity $\geq 2$ with $A$ at $p$.

Notice that the line in Example 2.2.4 is tangent to $C$ as $t$ has multiplicity 2. We also note in the following remark that we may express the defining polynomial $c(x, y)$ of $C$ in terms of the tangent line and higher degree terms.

Remark 2.2.6 Let $C$ be an affine curve defined by $c(x, y)$ and let $p=\left(x_{0}, y_{0}\right)$ be a nonsingular point on $C$. We may rewrite $c(x, y)$ in the following manner.

$c(x, y)=c_{0}\left(x-x_{0}, y-y_{0}\right)+c_{1}\left(x-x_{0}, y-y_{0}\right)+c_{2}\left(x-x_{0}, y-y_{0}\right)+\cdots+c_{n}\left(x-x_{0}, y-y_{0}\right)$

where the $c_{i}\left(x-x_{0}, y-y_{0}\right), i=1, \ldots, n$ are homogeneous polynomials of degree $i$. Notice that the linear term $c_{1}\left(x-x_{0}, y-y_{0}\right)=\frac{\partial c}{\partial x}(p)\left(x-x_{0}\right)+\frac{\partial c}{\partial y}(p)\left(y-y_{0}\right)$ is the equation of the tangent line to $C$ at $p$. Thus, at a nonsingular point $p$ we may express $c(x, y)$ in terms of the equation of the tangent line and higher degree terms.

We exemplify this remark in the following example.

Example 2.2.7 Let $C$ be an affine plane curve defined by the polynomial $c(x, y)$. Let $p=\left(x_{0}, y_{0}\right) \in C$. Let $L$ be a line through $p$. Then $L$ may be written in parametric form with parameter $t$, i.e

$$
x=x_{0}+\alpha t \quad y=y_{0}+\beta t
$$

for some $\alpha, \beta \in K$. We may express $c(x, y)$ in powers of $\left(x-x_{0}\right)$ and $\left(y-y_{0}\right)$ to get that

$$
c(x, y)=a\left(x-x_{0}\right)+b\left(y-y_{0}\right)+g(x, y)
$$

where $a=\frac{\partial c}{\partial x}(p), b=\frac{\partial c}{\partial x}(p)$ and $g(x, y)$ only consists of terms of degree $\geq 2$. Now to find the intersection multiplicity of $L$ on $C$ at $p$ we substitute the parameterization 
of $L$ into $c(x, y)$ to get that

$$
c\left(x_{0}+\alpha t, y_{0}+\beta t\right)=(a \alpha+b \beta) t+h(t)
$$

where the polynomial $h(t)$ contains only monomials of degree $\geq 2$. Recall that $a=$ $c_{x}^{\prime}\left(x_{0}, y_{0}\right)$ and $b=c_{y}^{\prime}\left(x_{0}, y_{0}\right)$, so we see that if $p$ is singular, i.e $a=b=0$, then all lines through $p$ are tangent. Alternatively, if $p$ is nonsingular then $L$ is tangent to $C$ at $p$ if and only if

$$
a \alpha+b \beta=0
$$

Thus if $p$ is non-singular then there is a unique line that is tangent to $C$ at $p$ which is defined by the polynomial $\frac{\partial c}{\partial x}(p)\left(x-x_{0}\right)+\frac{\partial c}{\partial y}(p)\left(y-y_{0}\right)$.

We now examine the tangent lines of a particular type of curve.

Example 2.2.8 Let $C$ be a curve defined by the polynomial

$$
c(x, y)=y^{2}-\varphi(x)=y^{2}-\prod_{i=0}^{n}\left(x-a_{i}\right)
$$

where all $a_{i} \in K$ are distinct. We examine the tangent lines at two types of points; when $y=0$ and when $y \neq 0$.

1. Let's first consider the points of $C$ where $y=0$. These are all the points of the form $\left\{\left(a_{i}, 0\right)\right\}$ for $i=1, \ldots, n$. In this case

$$
\begin{aligned}
& \frac{\partial c}{\partial x}\left(a_{i}, 0\right)=\varphi^{\prime}\left(a_{i}\right) \neq 0 \\
& \frac{\partial c}{\partial y}\left(a_{i}, 0\right)=2 y_{\mid y=0}=0 .
\end{aligned}
$$

Following Example 2.2.7, the tangent line $L$ to $C$ at $\left(a_{i}, 0\right)$ is defined by $\varphi^{\prime}\left(a_{i}\right)\left(x-a_{i}\right)$. Parameterizing $L$, we see that $L=(0,1) t+\left(a_{i}, 0\right)$, and sub- 
stituting this into $c(x, y)$ we obtain that

$$
c\left(a_{i}, t\right)=t^{2}+\varphi\left(a_{i}\right)=t^{2}
$$

as expected.

2. Let $p=\left(x_{0}, y_{0}\right) \in C$ where $y_{0} \neq 0$. Then

$$
\begin{aligned}
& \frac{\partial c}{\partial x}\left(x_{0}, y_{0}\right)=\varphi^{\prime}\left(x_{0}\right) \\
& \frac{\partial c}{\partial y}\left(x_{0}, y_{0}\right)=2 y_{0} \neq 0 .
\end{aligned}
$$

As in Example 2.2.7, the tangent line $L$ to $C$ at $\left(x_{0}, y_{0}\right)$ is defined by $l(x, y)=$ $\varphi^{\prime}\left(x_{0}\right)\left(x-x_{0}\right)+2 y_{0}\left(y-y_{0}\right)$. Parameterizing $L$, we get that $L=\left(\varphi^{\prime}\left(x_{0}\right), 2 y_{0}\right) t+$ $\left(x_{0}, y_{0}\right)$. Perhaps surprisingly, Definition 2.2 .5 tells us $c\left(\varphi^{\prime}\left(x_{0}\right) t+x_{0}, 2 y_{0} t+y_{0}\right)$ has $t=0$ as a root of degree $\geq 2$.

We now associate a closed set to tangent lines.

Definition 2.2.9 The collection of points on lines tangent to a closed affine set $A$ at $p$ is called the tangent space to $A$ at $p$. It is denoted by $\Theta_{p}$.

Example 2.2.10 From Example 2.2.7, the tangent line to $C$ at a nonsingular point $p=\left(x_{0}, y_{0}\right)$ is $\frac{\partial c}{\partial x}(p)\left(x-x_{0}\right)+\frac{\partial c}{\partial y}(p)\left(y-y_{0}\right)=0$. So

$$
\Theta_{p}=\left\{(x, y) \mid \frac{\partial c}{\partial x}(p)\left(x-x_{0}\right)+\frac{\partial c}{\partial x}(p)\left(y-y_{0}\right)=0\right\}
$$

Now, suppose that $p$ is a singular point of $C$. We saw in Example 2.2.7 that all lines through $p$ are tangent to $C$. Therefore $\Theta_{p}=\mathbb{A}^{2}$.

All of our examples up to this point have only dealt with affine curves. We will now look specifically at the projective case and demonstrate how it is a natural extension of the affine one. 
Example 2.2.11 Let $C \subset \mathbb{P}^{2}$ be a curve defined by the polynomial

$$
\bar{c}(X: Y: W)=Y^{2} W^{n-2}-\prod_{i=1}^{n}\left(X-a_{i} W\right)
$$

where all $a_{i} \in K$ are distinct. Dehomogenizing in $W$, we see that for all points with $W \neq 0, C$ may be identified with the affine curve defined by $c(x, y)=y^{2}-\prod_{i=0}^{n}\left(x^{i}-a_{i}\right)$ which we know to be nonsingular (Example 2.1.13. We know from Example 2.2.7 that the tangent line $L$ to a point $p=\left(x_{0}, y_{0}\right) \in C$ is defined by the equation

$$
\frac{\partial c}{\partial x}(p)\left(x-x_{0}\right)+\frac{\partial c}{\partial y}(p)\left(y-y_{0}\right)=0
$$

Homogenizing this equation in $W$, we have that $L$ is defined by

$$
\frac{\partial c}{\partial x}(p)\left(X-x_{0} W\right)+\frac{\partial c}{\partial y}(p)\left(Y-y_{0} W\right)=0 .
$$

Identifying $p$ with the projective point $p^{\prime}=\left(x_{0}: y_{0}: 1\right)$, and using the chain rule we see that $\frac{\partial c}{\partial x}(p)=\frac{\partial \bar{c}}{\partial X}\left(x_{0}: y_{0}: 1\right)$. Rearranging terms, and making this substitution, the equation of $L$ becomes

$$
\frac{\partial \bar{c}}{\partial X}\left(p^{\prime}\right) X+\frac{\partial \bar{c}}{\partial Y}\left(p^{\prime}\right) Y-\left(\frac{\partial \bar{c}}{\partial X}\left(p^{\prime}\right) x_{0}+\frac{\partial \bar{c}}{\partial Y}\left(p^{\prime}\right) y_{0}\right) W=0
$$

Now in general, for a homogeneous function $G$ of degree $n$, we have that

$$
\frac{\partial G}{\partial X} X+\frac{\partial G}{\partial Y} Y+\frac{\partial G}{\partial W} W=n G
$$

So for our point $p^{\prime}=\left(x_{0}: y_{0}: 1\right) \in C$,

$$
\frac{\partial \bar{c}}{\partial X}\left(p^{\prime}\right) x_{0}+\frac{\partial \bar{c}}{\partial Y}\left(p^{\prime}\right) y_{0}+\frac{\partial \bar{c}}{\partial W}\left(p^{\prime}\right)=n \cdot \bar{c}\left(p^{\prime}\right)=0 .
$$


Substituting this into our equation for $L$ we see that $L$ is defined by the equation

$$
\frac{\partial \bar{c}}{\partial X}\left(p^{\prime}\right) X+\frac{\partial \bar{c}}{\partial Y}\left(p^{\prime}\right) Y+\frac{\partial \bar{c}}{\partial W}\left(p^{\prime}\right) W=0
$$

This leads us to the following definition.

Definition 2.2.12 Let $C \subset \mathbb{P}^{N}$ be a projective closed set defined by the homogeneous polynomial $c(T)$. The tangent space to a point $p \in C$ is the set of points satisfying

$$
\sum_{i=0}^{N}\left(\frac{\partial c}{\partial T_{i}}(p)\right) T_{i}=0
$$

Example 2.2.13 Let $C \subset \mathbb{P}^{2}$ be a curve defined by the polynomial

$$
c(X: Y: W)=Y^{2} W^{n-2}-\prod_{i=1}^{n}\left(X-a_{i} W\right)
$$

where all $a_{i} \in K$ are distinct and $n \geq 3$. We now look at the tangent space to the points where $W=0$. There exists only one point, namely $p=(0: 1: 0)$, on $C$ for which $W=0$. Computing its tangent space we see that

$$
\begin{aligned}
\frac{\partial c}{\partial X}(p) & =\frac{\partial}{\partial X}\left(\prod_{i=0}^{n}\left(X^{i}-a_{i} W\right)\right)(p)=0 \\
\frac{\partial c}{\partial Y}(p) & =\frac{\partial}{\partial Y}\left(Y^{2} W^{n-2}\right)(p)=0 \\
\frac{\partial c}{\partial W}(p) & =\frac{\partial}{\partial W}\left(Y^{2} W^{n-2}\right)(p)-\frac{\partial}{\partial W}\left(\prod_{i=0}^{n}\left(X^{i}-a_{i} W\right)\right)(p) \\
& =\left((n-2) Y^{2} W^{n-3}\right)(p)+0
\end{aligned}
$$

Therefore for $n=3, \frac{\partial c}{\partial W}(p)=1$, and the tangent line $L$ to $C$ at $p$ is defined by the equation $W=0$. For $n>3, \frac{\partial c}{\partial W}(p)=0$, and so $p$ is singular. This implies that all lines through $p$ are tangent and thus $\Theta_{p}=\mathbb{P}^{2}$. 


\subsection{Valuations and the Local Ring at a Point}

To each point we define a local ring of functions defined at a point. We then restrict ourselves to nonsingular points, as their local rings have unique maximal principal ideals. We utilize the ideals of local rings at nonsingular points to define discrete valuations. Discrete valuations are functions from the function field of the curve to the integers. They provide a measure for the magnitude of a zero or a pole of a rational function at that point. Since every projective point is in some affine piece, and the pieces have isomorphic function fields, we may restrict ourselves to primarily the affine case. We begin with some ring theory. We reserve $A$ to denote an arbitrary irreducible closed set unless otherwise stated.

Definition 2.3.1 ([16] p.83) Let $A$ be an irreducible closed set and let $p \in A$. The local ring at $p$, denoted $\mathcal{O}_{p}$, is the subring of $K(A)$ consisting of all functions $g(t) / h(t) \in K(A)$ that are regular at $p$. i.e.

$$
\mathcal{O}_{p}=\left\{\frac{g(t)}{h(t)} \in K(A) \mid h(p) \neq 0\right\}
$$

Definition 2.3.2 Let $A$ be an irreducible closed set and let $p \in A$. The ideal at $p$, denoted $M_{p}$, is the kernel of the evaluation homomorphism at $p$ given by $E_{p}: \mathcal{O}_{p} \rightarrow K$ where $E_{p}(f(t))=f(p)$. Equivalently,

$$
M_{p}=\left\{\frac{g(t)}{h(t)} \in K(A) \mid g(p)=0\right\}
$$

Since $M_{p}$ is a kernel it is indeed an ideal. By the First Isomorphism Theorem $\mathcal{O}_{p} / M_{p}$ is isomorphic to the field $K$. Therefore $M_{p}$ is a maximal ideal.

Remark 2.3.3 Suppose that $\left(\frac{g(t)}{h(t)}\right)^{-1} \notin \mathcal{O}_{p}$. Without loss of generality we can assume that $h(t)$ and $g(t)$ have no common factors and so by Definition 2.3.1, it must be the case that $h(t)=0$, i.e. $\frac{g(t)}{h(t)} \in M_{p}$. Therefore $M_{p}$ is the set of non-units of $\mathcal{O}_{p}$, and as such it contains all other proper ideals of $\mathcal{O}_{p}$. Hence $M_{p}$ is the unique 
maximal ideal of $\mathcal{O}_{p}$. A ring with a unique maximal ideal is called local. This is why $\mathcal{O}_{P}$ is called the local ring at $p$. Furthermore, as $M_{p}$ contains all nonunits of $\mathcal{O}_{p}$, the set of units of $\mathcal{O}_{p}$ is $\mathcal{O}_{p} \backslash M_{p}$. This is the set of $u(t) \in \mathcal{O}_{p}$ such that $u(p) \neq 0$.

We now will continue to describe the properties of $\mathcal{O}_{p}$.

Lemma 2.3.4 ([4] p.44) Let $A$ be an irreducible closed affine set and let $p \in A$. Then the local ring $\mathcal{O}_{p}$ at $p$ is a Noetherian ring.

Proof. Let $I$ be an ideal of $\mathcal{O}_{p}$. We must show that the ideal $I$ of $\mathcal{O}_{p}$ is finitely generated. First, consider the ideal $K[A] \cap I$ of $K[A]$. As a Corollary to Hilbert's Basis Theorem ([2] p.637) we know that $K[A]$ is Noetherian. Therefore, there exists a set of generators $g_{1}(T), \ldots, g_{m}(T)$ such that $K[A] \cap I=\left(g_{1}(T), \ldots, g_{m}(T)\right)$. We will now show that $g_{1}(T), \ldots, g_{m}(T)$ generate $I$ as well. Suppose $f(T) \in I \subset \mathcal{O}_{p}$. Then $f(T)=g(T) / h(T)$ for some $g(T), h(T) \in K[A]$ such that $h(p) \neq 0$. Hence $h(T) f(T) \in K[A]$. Moreover, since $f(T) \in I$, we have that $h(T) f(T) \in K[A] \cap I$. Thus there exists $a_{i}(T) \in K[A], i=1, \ldots, m$, such that

$$
h(T) f(T)=\sum_{i=1}^{m} a_{i}(T) g_{i}(T)
$$

So

$$
f(T)=\sum_{i=1}^{m}\left(\frac{a_{i}(T)}{h(T)}\right) g_{i}(T)
$$

as desired.

We now show that the local rings at a point are invariant under isomorphism of curves.

Proposition 2.3.5 Let $C, D$ be irreducible curves. Let $p \in C$. Suppose that there exists an isomorphism $\Phi: C \rightarrow D$. Then the local rings $\mathcal{O}_{p}$ and $\mathcal{O}_{\Phi(p)}$ are isomorphic.

Proof. We will use our customary variable $T$ for functions on $C$ and we will use the variable $T^{\prime}$ for functions on $D$. Since $\Phi$ is an isomorphism it is a birational equivalence. 
Hence, by Theorem 2.1.75, there exists an isomorphism $\Phi^{*}: K(C) \rightarrow K(D)$ given by $\Phi^{*}(f(T))=f(\Phi(T))$. If we then restrict this isomorphism to $\mathcal{O}_{p}$ we need only check that $\Phi^{*}\left(\mathcal{O}_{p}\right)=\mathcal{O}_{\Phi(p)}$. For all $g\left(T^{\prime}\right) \in \mathcal{O}_{\Phi(p)}$ we have that $g(\Phi(p))=0$. This implies that

$$
0=\Phi^{-1}(g(\Phi(p)))=g\left(\Phi^{-1} \Phi(p)\right)=g(p) .
$$

Thus for all $g\left(T^{\prime}\right) \in \mathcal{O}_{\Phi(p)}$ there exists a function $g(T) \in \mathcal{O}_{p}$ such that $\Phi^{\prime}(g(T))=$ $g\left(T^{\prime}\right)$. Therefore $\Phi^{\prime}\left(\mathcal{O}_{p}\right)=\mathcal{O}_{\Phi(p)}$.

Example 2.3.6 Let $C$ be an affine plane curve defined by the polynomial $c(x, y)$. Let $p=\left(x_{0}, y_{0}\right)$ be a point on $C$. Then let $\Phi: \mathbb{A}^{2} \rightarrow \mathbb{A}^{2}$, be the regular map defined by $\Phi(x, y)=\left(x-x_{0}, y-y_{0}\right)$. We have that $\Phi$ is a linear shift and hence an isomorphism (Example 2.1.42). By Proposition 2.3.5, we may assume without loss of generality that $p=(0,0)$. Then $M_{p} \subset \mathcal{O}_{p}$, the maximal ideal of $p$, is generated by the functions $x$ and $y$, i.e. $M_{p}=(x, y)$. However, in the event that $p$ is non-singular, we will see in the following lemma that we may find a smaller generating set for $M_{p}$.

Lemma 2.3.7 ([4] p.70) Let $C$ be an affine plane curve defined by the polynomial $c(x, y)$. Let $p$ be a nonsingular point of $C$. Then $M_{p} \subset \mathcal{O}_{p}$ is a principal ideal.

Before we prove the lemma, to simplify the proof, we justify a few assumptions. As in Example 2.3.6, we can assume without loss of generality that $p=(0,0)$. Suppose that $p$ is nonsingular. Then, as in Example 2.2.7, there exists a unique tangent line $L$ to $C$ at $p$ given by the equation $\frac{\partial c}{\partial x}(p) x+\frac{\partial c}{\partial y}(p) y=0$. For the proof of the above lemma, we want $L$ to be defined by the polynomial $c y$ for some $0 \neq c \in K$. If $\frac{\partial c}{\partial x}(p)=0$ then the tangent line is already in the desired form. If not, we show that there exists a regular map on $C$, fixing $p$, that maps $L$ to the line defined by the polynomial $c y$ for some $0 \neq c \in K$. Define the linear map $\Phi: \mathbb{A}^{2} \rightarrow \mathbb{A}^{2}$ whereby $\Phi(x, y)=\left(y+\frac{\partial c}{\partial y}(p) \cdot x, \frac{\partial c}{\partial y}(p) \cdot x\right)$. Then $\Phi(0,0)=(0,0)$ and $\Phi(L)=\frac{\partial c}{\partial x}(p) y$ as desired. Since $\Phi$ is a regular map with a regular inverse $\Phi(x, y)=\left(x, x+\frac{\partial c}{\partial y}(p)\left(\frac{\partial c}{\partial x}\right)^{-1} y\right), C$ is isomorphic to the image $\Phi(C)$. By Proposition 2.3.5, the properties of the local 
ring at $p=(0,0)$ are preserved under $\Phi$. Therefore, we may assume without loss of generality that the tangent line to $p=(0,0)$ is defined by the equation $c y=0$. Furthermore, since $y$ defines the same line as $c y$, we may assume that the tangent line to $p=(0,0)$ is defined by the equation $y=0$.

Proof. Without loss of generality, we make the above assumptions that $p=(0,0)$ and that the tangent line to $L$ to $C$ at $p$ is defined by the equation $y=0$. As shown in Example 2.3.6, $M_{p}=(x, y)$ and so we need only prove that $y \in(x)$. From Remark 2.2.6, we may write $c(x, y)$ as the sum of the equation of the tangent line and higher degree terms, i.e.

$$
c(x, y)=y+g(x, y)
$$

Where $g(x, y)$ only contains monomials of degree $\geq 2$. Now, grouping together all the monomials with $y$ 's, we may express $c(x, y)$ as

$$
c(x, y)=y g_{1}(x, y)+g_{2}(x)
$$

where $g_{1}(x, y)=1+$ monomials of degree $\geq 1$, and $g_{2}(x) \in K[x]$ only contains monomials of degree $\geq 2$, i.e. we may rewrite this again as

$$
c(x, y)=y g_{1}(x, y)+x^{2} g_{3}(x)
$$

where $g_{3}(x) \in K[x]$. Then in $K[C], c(x, y)=0$ and as such

$$
y g_{1}(x, y)=-x^{2} g_{3}(x) \bmod I_{C} .
$$

Since $g_{1}(x, y)=1+$ terms of degree $\geq 1, g_{1}(0,0)=1 \neq 0$. Therefore $y=$ $g_{1}(x, y)^{-1} x^{2} g_{3}(x) \in(x) \subset \mathcal{O}_{p}$ and thus $M_{p}=(x)$.

We now give a definition of valuation rings with this property. 
Definition 2.3.8 Let $A$ be a closed set and let $p \in A$. Let $\mathcal{O}_{p}$ be the valuation ring at $p$ and let $M_{p}$ be the maximal ideal of $\mathcal{O}_{p}$. The $\mathcal{O}_{p}$ is called a discrete valuation ring if $M_{p}$ is principal.

As we have seen in Lemma 2.3.7, the valuation rings at non-singular points on plane affine curves are discrete valuation rings. We now state a more general result.

Theorem 2.3.9 Let $A$ be an affine closed set and let $p \in A$. Let $\mathcal{O}_{p}$ be the valuation ring at $p$ and let $M_{p}$ be the maximal ideal of $\mathcal{O}_{p}$. Then $\mathcal{O}_{p}$ is a discrete valuation ring if and only if $p$ is nonsingular.

We have proven one direction of this result for affine plane curves in Lemma 2.3.7. A complete proof of Theorem 2.3.9 is omitted as it requires an in-depth analysis of tangent spaces. For a complete proof see ([4] p.70).

Looking back at affine plane curves, we define the generator of the maximal ideal at a nonsingular point.

Definition 2.3.10 ([4] p.47) Let $C$ be an affine plane curve defined by the polynomial $c(x, y)$. Let $p$ be a nonsingular point of $C$. Then $M_{p}=(s(x, y)) \subset \mathcal{O}_{p}$ for some generator $s(x, y)$ in $K(C)$. Any element $s(x, y)$ where this is the case, is called a local parameter at $p$ or sometimes referred to as a uniformizing parameter.

Proposition 2.3.11 ([4] p.46) Let $C$ be an affine plane curve defined by the polynomial $c(x, y)$. Let $p$ be a nonsingular point of $C$. Let $s(x, y)$ be a local parameter at $p$, i.e. $(s(x, y))=M_{p} \subset \mathcal{O}_{p}$. Then for all $z(x, y) \in \mathcal{O}_{p}$ there exists a unit $u(x, y) \in \mathcal{O}_{p}$ and a non-negative integer $m$ such that $z(x, y)$ may be expressed uniquely in the form $z(x, y)=u(x, y) s(x, y)^{m}$.

Proof. We first prove the uniqueness. Suppose that

$$
z(x, y)=u(x, y) s(x, y)^{n}=v(x, y) s(x, y)^{m}
$$


where $u(x, y)$ and $v(x, y)$ are units. Without loss of generality suppose $n \geq m$. Then $s(x, y)^{n-m}=u(x, y)^{-1} v(x, y)$ is a unit. However, $s(x, y) \in M_{p}$ is not a unit as it generates a proper ideal, and so it must be the case that $n=m$ and consequently $u=v$.

We now prove that the expression $z(x, y)=u(x, y) s(x, y)^{m}$ exists. We first note that if $z(x, y)$ is a unit then we may write $z(x, y)=z(x, y) s(x, y)^{0}$. Assuming that $z(x, y)$ is not a unit, then since $M_{p}$ contains all non-units (Remark 2.3.3), $z(x, y) \in$ $M_{p}=(s(x, y))$, i.e. $z(x, y)=z_{1}(x, y) s(x, y)$ for some $z_{1}(x, y) \in \mathcal{O}_{p}$. If $z_{1}(x, y)$ is a unit then we are done. Otherwise, $z_{1}(x, y) \in(s(x, y))$ and so $z_{1}(x, y)=z_{2}(x, y) s(x, y)$. If $z_{2}(x, y)$ is a unit then $z(x, y)=z_{2}(x, y) s(x, y)^{2}$ and we are done. Suppose we continue on this way and suppose further that $z_{i}(x, y)$ is not a unit for all $i=1,2, \ldots$ where $z_{i}(x, y)=z_{i+1}(x, y) s(x, y)$. We may then construct an infinite chain of ideals $\left(z_{1}(x, y)\right) \subseteq\left(z_{2}(x, y)\right) \subseteq\left(z_{3}(x, y)\right) \subseteq \ldots$ However, since $\mathcal{O}_{p}$ is Noetherian this chain must have a maximal element ([2] p.637), i.e. $\left(z_{k}(x, y)\right)=\left(z_{k+1}(x, y)\right)$ for some $k$. This implies that $z_{k+1}(x, y)=v(x, y) z_{k}(x, y)$ for some $v \in \mathcal{O}_{p}$. Consequently, $z_{k}(x, y)=v(x, y) s(x, y) z_{k}(x, y)$ and so $1=v(x, y) s(x, y)$. This is a contradiction since $s(x, y)$ is not a unit. Thus there must exist some $n$ for which our process terminates, i.e. there exists an $n$ such that $z_{n}(x, y)$ is a unit. Consequently we may express $z(x, y)$ as $z(x, y)=z_{n}(x, y) s(x, y)^{n}$.

For a nonsingular point $p$ on a plane curve $C$, we have seen in Lemma 2.3.7 that $M_{p}$ is a principal ideal and hence that local parameters exist. However, we have not yet seen any examples of local parameters. We will now describe an entire class of local parameters for a given point $p$.

Proposition 2.3.12 Let $C$ be an affine plane curve defined by the polynomial $c(x, y)$. Let $p$ be a nonsingular point of $C$. Let $L_{2}$ be any line through $p$ that is not tangent to $C$ at $p$. Then the defining polynomial of $L_{2}$ is a local parameter at $p$.

Proof. Let $C$ be an affine plane curve and let $p \in C$ be nonsingular. As in the proof of Lemma 2.3.7, we may assume without loss of generality that $p=(0,0)$ and 
that the tangent line $L$ to $C$ at $p$ is defined by the equation $y=0$. We will now show that there exists a regular map which fixes both the tangent line $L$ defined by $y=0$ and the point $p=(0,0)$. This map will also send any other fixed line $L_{2}$ through $p$, that is not tangent to $C$ at $p$, to the line defined by $x=0$. Suppose that $L_{2}=a x+b y$. Since $L_{2}$ is not tangent to $p, a \neq 0$. Define the map $\Phi: \mathbb{A}^{2} \rightarrow \mathbb{A}^{2}$ by $\Phi(x, y)=\left(a^{-1} x-a^{-1} b y, y\right)$. Then $\Phi(0,0)=(0,0), \Phi(L)=L$ and $\Phi\left(L_{2}\right)$ equals the line defined by $x=0$. Recall from the proof of Lemma 2.3.7 that $M_{p}=(x)$. Thus $\Phi$ maps the defining equation of $L_{2}$ to a local parameter. Since $\Phi$ is a regular map, $C$ is isomorphic to the image $\Phi(C)$. By Proposition 2.3.5, the properties of the local ring at $p=(0,0)$ are preserved under $\Phi$. Hence, the defining equation of any line through $p$, that is not tangent to $C$, is a local parameter at $p$.

There is a strong relationship between two local parameters at a nonsingular point.

Proposition 2.3.13 Let $C$ be an affine plane curve and let $p$ be a nonsingular point of $C$. Suppose that $s(x, y)$ and $s^{\prime}(x, y)$ are two local parameters at $p$. Then there exists a unit $u(x, y) \in \mathcal{O}_{p}$ such that $s^{\prime}(x, y)=u(x, y) s(x, y)$.

Proof. Suppose that $s(x, y)$ and $s^{\prime}(x, y)$ are two local parameters at $p$. Then there exist a unit $u(x, y)$ such that $s^{\prime}(x, y)=u(x, y) s(x, y)^{n}$ for some $n>0$ (Proposition 2.3.11). Similarly, there exists a unit $u^{\prime}(x, y)$ such that $s(x, y)=u^{\prime}(x, y) s^{\prime}(x, y)^{m}$ for some $m>0$. Combining these statements we obtain that $s=u^{\prime}\left(u s^{n}\right)^{m}$. By the uniqueness in Proposition 2.3.11, it must be the case that $n=m=1$. Thus two local parameters differ only by the product of a unit.

Example 2.3.14 Let $C$ be an affine plane curve defined by the polynomial $y^{2}-\varphi(x)$ and let $p=\left(x_{0}, y_{0}\right) \in C$. We have seen in Example 2.2.8, that if $y_{0}=0$ then the tangent line to $C$ at $p$ is defined by the polynomial $x-x_{0}$. Hence $y$ is not tangent to $p$. For points of this type it is typical to take $y$ as a local parameter. If $y_{0} \neq 0$ then the tangent line to $p$ at $C$ is defined by the polynomial $\varphi^{\prime}\left(x_{0}\right)\left(x-x_{0}\right)+2 y_{0}\left(y-y_{0}\right)$. Hence $x-x_{0}$ is not tangent to $C$ at $p$ and is typically taken to be a local parameter. 
We can now represent functions in terms of local parameters. Using local parameters, we explore ways to compare and measure how functions behave at nonsingular points. We begin with some properties of local rings at a point.

Definition 2.3.15 Let $A$ be a closed set. A valuation ring of a rational function field $K(A)$ is a ring $\mathcal{O}$ such that $K \varsubsetneqq \mathcal{O} \varsubsetneqq K(A)$ and for any $g(t) / h(t) \in K(A)$, $g(t) / h(t) \in \mathcal{O}$ or $h(t) / g(t) \in \mathcal{O}$.

Proposition 2.3.16 Let $C$ be an affine plane curve. The local ring $\mathcal{O}_{p}$ at a nonsingular point $p$ is a valuation ring.

Proof. Let $g(x, y) / h(x, y) \in K(C)$. Let $s(x, y)$ be a local parameter at $p$. From Proposition 2.3.11, we may write $g(x, y)=u_{g}(x, y) s(x, y)^{n}$ and $h(x, y)=$ $u_{h}(x, y) s(x, y)^{m}$ for some units $u_{h}(x, y), u_{g}(x, y) \in \mathcal{O}_{p}$ and positive integers $n$ and $m$. Suppose that $g(x, y) / h(x, y) \notin \mathcal{O}_{p}$. This means that

$$
\frac{g(x, y)}{h(x, y)}=\frac{u_{g}(x, y) s(x, y)^{n}}{u_{h}(x, y) s(x, y)^{m}}=\frac{u_{g}(x, y)}{u_{h}(x, y)} s(x, y)^{n-m} \notin \mathcal{O}_{p}
$$

Since $u_{g}(x, y)$ and $u_{h}(x, y)$ are units, $u_{g}(p) \neq 0$ and $u_{h}(p) \neq 0$. Since $s(p)=0$, $g(x, y) / h(x, y) \notin \mathcal{O}_{p}$ implies that $n>m$. Now taking the inverse of $\frac{g(x, y)}{h(x, y)}$ and evaluating at $p$ we have that

$$
\left(\frac{g(p)}{h(p)}\right)^{-1}=\frac{h(p)}{g(p)}=\frac{u_{h}(p)}{u_{g}(p)} s(x, y)^{m-n}=0 .
$$

Therefore $\frac{h(x, y)}{g(x, y)} \in \mathcal{O}_{p}$.

Lemma 2.3.17 Let $C$ be a plane affine curve and let $p \in C$ be nonsingular. Let $s(x, y)$ be a local parameter at $p$. For any $z(x, y) \in K(C), z(x, y)=u(x, y) s(x, y)^{n}$, for some $u(x, y) \in \mathcal{O}_{p}$ a unit, and some $n \in \mathbb{Z}$.

Proof. If $z(x, y)$ is in $\mathcal{O}_{p}$ then this has been proven in Proposition 2.3.11. If $z(x, y) \notin$ $\mathcal{O}_{p}$ then $z(x, y)^{-1} \in \mathcal{O}_{p}$ by Proposition 2.3.16. Hence there exists a unit $u(x, y) \in \mathcal{O}_{p}$ 
such that $z^{-1}=u(x, y) s(x, y)^{n}$ for some nonnegative integer $n$. Inverting both sides, we attain that $z=u^{-1} s^{-n}$ as desired.

We now define a function to analyze the behavior of a rational function at nonsingular point. We begin with a general definition.

Definition 2.3.18 Let $A$ be a closed affine set. A discrete valuation on $K(A)$ is a function $v: K(A) \rightarrow \mathbb{Z} \cup \infty$ such that

1. $v(g(t))=\infty$ if and only if $g(t)=0$

2. $v(g(t) h(t))=v(g(t))+v(h(t))$ for all $g(t), h(t) \in K(A)$

3. $v(g(t)+h(t)) \geq \min \{v(g(t)), v(h(t))\}$ for all $g(t), h(t) \in K(A)$

4. $v(k)=0$ for any $0 \neq k \in K$.

We will also need to make use of the following property.

Lemma 2.3.19 The Strict Triangle Inequality ([19] p.5) Let $v$ be a discrete valuation of $K(C)$ and let $a(t), b(t) \in K(C)$ be such that $v(a(t)) \neq v(b(t))$. Then $v(a(t)+b(t))=\min \{v(a(t)), v(b(t))\}$.

Proof. Since $v(a(t)) \neq v(b(t))$ we may assume $v(a(t))<v(b(t))$. Suppose that $v(a(t)+b(t)) \neq \min \{v(a(t)), v(b(t))\}$, and so $v(a(t)+b(t))>v(a(t))$. We obtain that $v(a)=v((a(t)+b(t))-b(t)) \geq \min \{v(a(t)+b(t)), v(b(t))\}>v(a(t))$, a contradiction.

We now define some particular discrete valuations associated to a plane affine curve.

Definition 2.3.20 Let $C$ be an affine plane curve and let $p \in C$ be nonsingular. Let $s(x, y)$ be a local parameter at $p$. From Proposition 2.3.17 we know that for any 
function $z(x, y) \in K(C), z(x, y)=u(x, y) s(x, y)^{n}$ for some unique unit $u(x, y) \in \mathcal{O}_{p}$, $n \in \mathbb{Z}$. The valuation at $p$ is a function $v_{p}: K(C) \rightarrow \mathbb{Z} \cup \infty$ such that

$$
v_{p}(z(x, y))=\left\{\begin{array}{lr}
\infty, & \text { if } z(x, y)=0 \\
n, & \text { otherwise }
\end{array}\right.
$$

It may not be obvious that $v_{p}$ is well-defined. Recall from Proposition 2.3.13, that given any two local parameters $s(x, y)$ and $r(x, y)$, there exists a unit $w(x, y)$ such that $s(x, y)=r(x, y) w(x, y)$. Hence given the representations $z(x, y)=u(x, y) s(x, y)^{n}$, and $z(x, y)=v(x, y) r(x, y)^{m}$ we may substitute in for $s(x, y)$ to obtain that $u(x, y) s(x, y)^{n}=v(x, y) w(x, y)^{m} s(x, y)^{m}$. Assuming that $n \geq m$, it must be the case that $s^{n-m}$ is a unit, i.e. $n=m$. Therefore the valuation function at $p$ is well-defined.

Note that by definition, units have valuation 0 and local parameters have valuation one.

In the next two examples we describe the valuations at every point of an affine plane curve defined by $y^{2}=\varphi(x)$. In the example following these two, we describe the valuation at the new point added when considering the projective closure, i.e. the "point at infinity".

Example 2.3.21 Let $C$ be an affine plane curve defined by $y^{2}-\varphi(x)$. From Example 2.3 .14 we know that $y$ is a local parameter at all points $\left(x_{0}, 0\right) \in C$. Since $x_{0}$ is a root of $\varphi(x)$ and $\varphi(x)$ has no repeated roots (Remark 2.1.36) we may write

$$
\varphi(x)=\left(x-x_{0}\right) g(x) \quad \text { where } g\left(x_{0}\right) \neq 0 \text {. }
$$

Taking the valuation of both sides we see that

$$
v_{p}(\varphi(x))=v_{p}\left(\left(x-x_{0}\right) g(x)\right)=v_{p}\left(x-x_{0}\right)+v_{p}(g(x))=v_{p}\left(x-x_{0}\right) .
$$

We use this identity to find the valuation of $x-x_{0}$. Taking the valuation at $p$ we see 
that

$$
v_{p}\left(x-x_{0}\right)=v_{p}(\varphi(x))=v_{p}\left(y^{2}\right)=2 v_{p}(y)=2 \cdot 1=2 .
$$

Now, given any rational function $z(x, y) \in K(C)$, we know from Remark 2.1.52 that $z(x, y)$ may be expressed as

$$
z(x, y)=\frac{a(x)+b(x) y}{d(x)} \quad \text { for some } a(x), b(x), d(x) \in K[C] .
$$

To find the valuation of $z(x, y)$ we look at each term of the function on the right. We may write $a(x)=\left(x-x_{0}\right)^{m_{a}} a_{1}(x)$ where $a_{1}\left(x_{0}\right) \neq 0$. Hence

$$
v_{p}(a(x))=m_{a} \cdot v_{p}\left(x-x_{0}\right)+v_{p}\left(a_{1}(x)\right)=m_{a} \cdot 2+0=2 m_{a} .
$$

Similarly, we may write $d(x)=\left(x-x_{0}\right)^{m_{d}} d_{1}(x)$ where $d_{1}\left(x_{0}\right) \neq 0$ and the same calculation gives us that $v_{p}(d(x))=2 m_{d}$. Finally, we may write $b(x)=\left(x-x_{0}\right)^{m_{b}} b_{1}(x)$ where $b_{1}\left(x_{0}\right) \neq 0$ to see that valuation of the $b(x) y$ is

$$
v_{p}(b(x) y)=m_{b} \cdot v_{p}\left(x-x_{0}\right)+v_{p}(y)=m_{a} \cdot 2+1=2 m_{a}+1 .
$$

Putting all of this together, we may calculate the valuation of $z$,

$$
v_{p}(z(x, y))=v_{p}\left(\frac{a(x)+b(x) y}{d(x)}\right)=v_{p}(a(x)+b(x) y)-v_{p}(d(x)) .
$$

Since $v_{p}(b(x) y)=2 m_{b}+1$ is odd and $v_{p}(a(x))=2 m_{a}$ is even we may apply the Strict Triangle Inequality (Lemma 2.3.19) to obtain

$$
v_{p}(z)=\min \left\{v_{p}\left(a(x), v_{p}(b(x) y)\right\}-v_{p}(d(x))=\min \left\{2 m_{a}, 2 m_{b}+1\right\}-2 m_{d} .\right.
$$

Example 2.3.22 Let $C$ be an affine plane curve defined by $y^{2}=\varphi(x)$. From Example 2.3.14 we know that $x-x_{0}$ is a local parameter at all points $\left(x_{0}, y_{0}\right) \in C$ where $y_{0} \neq 0$. Now, given any rational function $z \in K(C)$, we know from Remark 2.1.52 that $z(x, y)$ 
may be expressed as

$$
z(x, y)=\frac{a(x)+b(x) y}{d(x)} \quad \text { for some } a(x), b(x), d(x) \in K[C] .
$$

To find the valuation of $z(x, y)$ we again look at each term of the function. As in Example 2.3.21 we may rewrite $a(x), b(x)$ and $d(x)$ such that

$$
\begin{array}{r}
a(x)=\left(x-x_{0}\right)^{m_{a}}\left(a_{1}(x)\right) \text { where } a_{1}\left(x_{0}\right) \neq 0, \\
b(x)=\left(x-x_{0}\right)^{m_{b}}\left(b_{1}(x)\right) \text { where } b_{1}\left(x_{0}\right) \neq 0, \\
d(x)=\left(x-x_{0}\right)^{m_{d}}\left(d_{1}(x)\right) \text { where } d_{1}\left(x_{0}\right) \neq 0 .
\end{array}
$$

We first consider the case that $m_{b}>m_{a}$. Combining the terms and factoring we obtain that

$$
\begin{aligned}
v_{p}(z) & =v_{p}\left(\frac{a(x)+b(x) y}{d(x)}\right) \\
& =v_{p}\left(\left(x-x_{0}\right)^{m_{a}-m_{d}} \frac{a_{1}(x)+b_{1}(x)\left(x-x_{0}\right)^{m_{b}-m_{a} y}}{d_{1}(x)}\right) \\
& =\left(m_{a}-m_{d}\right)+v_{p}\left(\frac{a_{1}(x)+b_{1}(x)\left(x-x_{0}\right)^{m_{b}-m_{a}} y}{d_{1}(x)}\right) .
\end{aligned}
$$

Notice that when we evaluate at $\left(x_{0}, y_{0}\right)$

$$
\frac{a_{1}\left(x_{0}\right)+b_{1}\left(x_{0}\right)(0)^{m_{b}-m_{a}} y_{0}}{d_{1}\left(x_{0}\right)}=\frac{a_{1}\left(x_{0}\right)}{d_{1}\left(x_{0}\right)} \neq 0
$$

Hence,

$$
v_{p}\left(\left(x-x_{0}\right)^{m_{a}-m_{d}} \frac{a_{1}(x)+b_{1}(x)\left(x-x_{0}\right)^{m_{b}-m_{a}} y}{d_{1}(x)}\right)=\left(m_{a}-m_{d}\right)
$$

For the case that $m_{b}<m_{a}$, a similar calculation shows that $v_{p}(z)=m_{b}-m_{d}$. Finally, 
for the case $m_{b}=m_{a}$ we have that

$$
\begin{aligned}
v_{p}(z) & =v_{p}\left(\frac{a(x)+b(x) y}{d(x)}\right) \\
& =v_{p}\left(\left(x-x_{0}\right)^{m_{a}-m_{d}} \frac{a_{1}(x)+b_{1}(x) y}{d_{1}(x)}\right) \\
& =\left(m_{a}-m_{d}\right)+v_{p}\left(\frac{a_{1}(x)+b_{1}(x) y}{d_{1}(x)}\right) .
\end{aligned}
$$

However, unlike our the previous two cases, it could be the case that $a_{1}\left(x_{0}\right)+$ $b_{1}\left(x_{0}\right) y_{0}=0$. We know that this function may be expressed in the form $u(x, y)(x-$ $\left.x_{0}\right)^{m}$ for some $m \geq 0$, but it is not completely obvious how one would do this. There does exist an algorithm that accomplishes this but it would require a rather lengthy discussion to describe and prove (see [9] for an algorithm to do this). We may avoid this issue by using completions, a topic that will be discussed in Section 3.4. We will see that by using completions we are able to completely describe the valuations of all rational functions.

We will now define valuations on projective plane curves. However, to do so we need to make use of the following lemma.

Lemma 2.3.23 Let $C$ be a projective plane curve. Let $p$ be a non-singular point of $C$. Then $p$ is in some affine piece $U_{i}$ and there exists an affine closed set $A_{i}$ isomorphic to the $U_{i}$ where the image of $p$ is nonsingular.

Proof. Suppose that $C$ is defined by the homogeneous polynomial $c(X: Y: Z)$. Since $p \in \mathbb{P}^{2}$ one of the entries of $p$ is nonzero. As a change of variables is an isomorphism, (Defintion 2.1.43) without loss of generality, we assume that $p_{3}$ is nonzero, i.e. $p \in U_{3}$. This implies that we may write $p$ as $p=\left(p_{1}: p_{2}: 1\right)$. Since $p$ is nonsingular, by Definition 2.1.18, at least one of $\frac{\partial c}{\partial X}(p), \frac{\partial c}{\partial Y}(p), \frac{\partial c}{\partial Z}(p)$ is nonzero. We now consider two cases.

1. Suppose that at least one of $\frac{\partial c}{\partial X}(p), \frac{\partial c}{\partial Y}(p)$ is non-zero. We know that $U_{3}$ is isomorphic to the affine place curve $A$ defined by the polynomials $a(x, y)=$ 
$c(x, y, 1)$ where the image of $p$ is $p_{A}=\left(p_{1}, p_{2}\right)$. Furthermore, as we saw in Example 2.2.11, $\frac{\partial a}{\partial x}\left(p_{A}\right)=\frac{\partial c}{\partial X}(p)$ and $\frac{\partial a}{\partial y}\left(p_{A}\right)=\frac{\partial c}{\partial Y}(p)$. By assumption, one of these partial derivatives is nonzero. Therefore the image of $p$ is nonsingular.

2. Suppose that $\frac{\partial c}{\partial X}(p)=\frac{\partial c}{\partial Y}(p)=0$, i.e. the equation of the tangent line at $p$ is $\frac{\partial c}{\partial Z}(p) Z=0$. Applying the isomorphism $\phi$ where $\phi(Z)=Z+Y$, we have that the equation of the tangent line becomes $\frac{\partial c}{\partial Z}(p) Z+\frac{\partial c}{\partial Z}(p) Y=0$. However, in general we know that the equation of the tangent line at $p$ is $\frac{\partial \phi(c)}{\partial X}(p) X+$ $\frac{\partial \phi(c)}{\partial Y}(p) Y+\frac{\partial \phi(c)}{\partial Z}(p) Z=0$. Therefore, after the applying the isomorphism, it must be the case that $\frac{\partial c}{\partial Z}(p)=\frac{\partial \phi(c)}{\partial Y}(p) \neq 0$. Thus we are in case 1 .

All cases being considered, we have shown the desired result.

Definition 2.3.24 Let $C$ be a projective plane curve. Then for all $p \in C$, there exists an affine piece $U_{i}$ such that $p \in U_{i}$ (Definition 2.1.21). By Lemma 2.3.23, there exists an affine plane curve $A_{i}$ isomorphic to $U_{i}$ where $p_{i} \in A_{i}$ is the nonsingular image of $p$. Then for all $F(T) \in K(C)$ we write $f_{i}(t)$ for the image of $F$ in $K\left(A_{i}\right)$. We define the valuation of $F(T)$ at the nonsingular projective point $p$ as $v_{p}(F(T))$ where $v_{p}(F(T))=v_{p_{i}}\left(f_{i}(t)\right)$.

It may not be obvious that $v_{p}$ is well-defined. However, Proposition 2.3.5 guarantees that at any two such isomorphic $A_{i}$ also have isomorphic local rings. Thus Definition 2.3 .24 is indeed well-defined.

Definition 2.3.25 Let $C$ be a curve (affine or projective). Let $p$ be a nonsingular point of $C$. We say that a function $g(T) \in K(C)$ has a zero at $p$ if $v_{p}(g(T))>0$. Alternatively, we say that $g(T)$ has a pole at $p$ if $v_{p}(g(T))<0$.

In the following example, to make distinctions between the coordinates in different affine pieces we use subseripts to denote the variables by which dehomogenization occurred. 
Example 2.3.26 Let $C$ be a affine plane curve defined by the polynomial $y^{2}-\varphi(x)$. From Example 2.2.13 we know that the projective closure is nonsingular only if the $\operatorname{deg}(\varphi(x))=3$. In this example we will restrict ourselves to this case.

Let $\bar{C}$ be the projective closure of $C$ defined by the homogeneous polynomial

$$
Y^{2} Z-\bar{\varphi}(X: Z)
$$

The valuations of $\bar{C}$ at all points $(X: Y: Z)$ with $Z \neq 0$ are defined to be equal to the valuations of the corresponding point of $C$. Thus, the only new point to consider is the point $p_{\infty}=(0: 1: 0)$. Let $C_{2}$ denote the affine piece of $\bar{C}$ obtained by dehomogenizing in $Y$. Dehomogenizing $p_{\infty}$ we obtain that $p_{\infty} \mapsto(0,0) \in C_{2}$. We will use $y_{z}$ and $x_{z}$ for the coordinates of $C$ and $x_{y}, z_{y}$ for the coordinates of $C_{2}$. With this notation, $C_{2}$ is defined by the polynomial $c_{2}\left(x_{y}, z_{y}\right)=z_{y}-\bar{\varphi}\left(x_{y}, z_{y}\right)$ obtained by dehomogenizing Equation (2.5). Notice that indeed $(0,0) \in C_{2}$. Finding the tangent line at $(0,0)$, we take partial derivatives and see that

$$
\frac{\partial c_{2}}{\partial z_{y}}(0,0)=1-\frac{\partial \overline{\varphi^{\prime}}}{\partial z_{y}}(0,0) \quad \text { and } \quad \frac{\partial c_{2}}{\partial x_{y}}(0,0)=0-\frac{\partial \overline{\varphi^{\prime}}}{\partial x_{y}}(0,0)
$$

Since $\bar{\varphi}\left(x_{y}, z_{y}\right)$ is a homogeneous polynomial of degree $3, \frac{\partial \varphi^{\prime}}{\partial z_{y}}(0,0)=\frac{\partial \overline{\varphi^{\prime}}}{\partial x_{y}}=(0,0)=0$. We know from Example 2.2.7 that the equation to the tangent line at $p$ is

$$
\frac{\partial c_{2}}{\partial z_{y}}(p) \cdot z_{y}+\frac{\partial c_{2}}{\partial x_{y}}(p) \cdot x_{y}
$$

Hence, the tangent line to $C_{2}$ at $(0,0)$ is

$$
\frac{\partial c_{2}}{\partial z_{y}}(p) \cdot z_{y}+\frac{\partial c_{2}}{\partial x_{y}}(p) \cdot x_{y}=z_{y}
$$

Since $(0,0)$ is a nonsingular point, from Proposition 2.3.12, the defining polynomial of any line through $(0,0)$ which is not tangent is a local parameter. Hence $x_{y}$ is a local parameter. 
We now compute the valuation of $z_{y}$. We begin by noting that since $\bar{\varphi}(x, z)$ is a homogeneous polynomial of degree 3 it may be written as

$$
\bar{\varphi}\left(x_{y}, z_{y}\right)=a_{1} x_{y}^{3}+a_{2} x_{y}^{2} z_{y}+a_{3} x_{y} z_{y}^{2}+a_{4} z_{y}^{3}
$$

For some $a_{1}, a_{2}, a_{3}, a_{4} \in K$. Since $\bar{\varphi}(x, z)$ is the homogenization of $\varphi(x)$ which is of degree 3 by assumption, we know that $a_{1} \neq 0$ by assumption. Using this information, we rewrite the equation $c_{2}\left(x_{y}, z_{y}\right)=0$ to obtain

$$
\begin{aligned}
0 & =z_{y}-\bar{\varphi}\left(x_{y}, z_{y}\right), \\
0 & =z_{y}-a_{1} x_{y}^{3}+a_{2} x_{y}^{2} z_{y}+a_{3} x_{y} z_{y}^{2}+a_{4} z_{y}^{3}, \\
a_{1} x_{y}^{3} & =z_{y}\left(1-a_{2} x_{y}^{2}+a_{3} x_{y} z_{y}+a_{4} z_{y}^{2}\right) .
\end{aligned}
$$

Since $1-a_{2} x_{y}^{2}+a_{3} x_{y} z_{y}+a_{4} z_{y}^{2}$ evaluated at $(0,0)$ is 1 , we have that

$$
\begin{aligned}
v_{p_{\infty}}\left(a_{1} x_{y}^{3}\right) & =v_{p_{\infty}}\left(z_{y}\left(1-a_{2} x_{y}^{2}+a_{3} x_{y} z_{y}+a_{4} z_{y}^{2}\right)\right) \\
3 v_{p_{\infty}}\left(x_{y}\right) & =v_{p_{\infty}}\left(z_{y}\right) \\
3 & =v_{p_{\infty}}\left(z_{y}\right) .
\end{aligned}
$$

Finally, mapping back to $C$, the original affine plane curve, we have that

$$
z_{y}=Z / Y=1 / y_{z} \quad \text { and } \quad x_{y}=X / Y=x_{z} / y_{z}
$$

Taking valuations of both sides we obtain that

$$
3=v_{p_{\infty}}\left(z_{y}\right)=v_{p_{\infty}}\left(1 / y_{z}\right) \quad \text { and } \quad v_{p_{\infty}}\left(x_{y}\right)=v_{p_{\infty}}\left(x_{z}\right)-v_{p_{\infty}}\left(y_{z}\right)
$$

So we have that $v_{p_{\infty}}\left(y_{z}\right)=-3, v_{p_{\infty}}\left(x_{z}\right)=-2$ and $x_{z} / y_{z}$ is a local parameter at $p_{\infty}$.

Let $C$ be a projective plane curve. Suppose at a point $p \in C$ the valuation of 
$v_{p}(x)=a<0$. Let $0<m \in \mathbb{Z}$ and notice that $v_{p}\left(x^{m}\right)=m v_{p}(x)<v_{p}(x)$. Applying $v_{p}$ to a polynomial $g(x) \in K(C)$ of degree $n$, by the Strict Triangle Inequality $v_{p}(g(x))=$ $v_{p}\left(x^{n}\right)=n \cdot v_{p}(x)$. This is exactly the case of $v_{p_{\infty}}$ on $\bar{C}$ in the above example.

For the projective closure of curves defined by $y^{2}-\varphi(x)$ with $\operatorname{deg}(\varphi(x))>3$, the point $p=(0: 1: 0)$ is singular. As such, its local ring is not necessarily principal and we cannot define a valuation for this point. We will explore this problem further in the next two sections.

\subsection{Nonsingular Models}

This section is designed to give the reader an intuition into the following theorem and some of its consequences. From this point forward, all curves are assumed to be irreducible unless otherwise stated.

Theorem 2.4.1 ([4] p.179) Let $C$ be a projective plane curve. Then there exists a nonsingular curve $C^{\prime} \subset \mathbb{P}^{n}$ such that $C^{\prime}$ is birationally equivalent to $C$.

The proof of this theorem is constructive in nature, i.e. given a projective plane curve $C$ one performs some birational maps to obtain a nonsingular curve. In the proof, two different types of maps are required, blow-ups and quadratic transformations. We will define each of these maps, but omit the proof of the theorem, as it requires a rather lengthy analysis of each of these maps. We will explore some consequences of the theorem later on.

Definition 2.4.2 The standard quadratic transformation is the map $Q: \mathbb{P}^{2} \rightarrow \mathbb{P}^{2}$ where $Q(X: Y: Z)=(Y Z: X Z: X Y)$.

By Defintion 2.1.66 $Q$ is a rational map. We see that $Q(Q(X: Y: Z))=$ $X Y Z(X: Y: Z)=(X: Y: Z)$ and thus it is its own inverse. Moreover, it is regular at all points except $(1: 0: 0),(0: 1: 0)$, and $(0: 0: 1)$, which get mapped to $0 \notin \mathbb{P}^{2}$. In the constructive proof, a quadratic transformations are used to change the types of singularities from "cusps" to "nodes" (see [4] for further explanation). 
We now present the affine version of a blow-up.

Definition 2.4.3 Let $B \subset \mathbb{A}^{3}$ be the closed set defined by the equation $y=x z$. A blowup centered at $(0,0)$ is the map $\beta: \mathbb{A}^{2} \rightarrow B$ where $\beta(x, y)=(x, x z, z)$.

So for an affine plane curve $C$ defined by the polynomial $c(x, y), \beta(C)$ is the closed set defined by the common zeros of $c(x, y)$ and $y=x z$ in $\mathbb{A}^{3}$. A blow-up is also a rational map, it is regular at all points different from $(0,0)([4]$ p.162). In the constructive proof, blowups map "nodes" to distinct points. These points may still be singular, but they're multiplicity is lowered (see [4] for further explanation).

Theorem 2.4.1 is essentially a corollary to the following lemma

Lemma 2.4.4 ([4] p.179) Let $C$ be a projective plane curve. By applying a finite series of quadratic transformations, dehomogenizations, blowups and homogenizations, we obtain a nonsingular projective curve birationally equivalent to $C$.

Hence, in a sense, one applies quadratic transformations to turn singularities into the right type, then uses blowups to lessen their multiplicity, and repeats as necessary. For more thorough examples on blow-ups and quadratic transformations see [13].

We now explore some properties of nonsingular projective curves.

Theorem 2.4.5 ([16] p.109) Let $C$ be an irreducible projective curve and let $\Phi: C \rightarrow$ $\mathbb{P}^{n}$ be a rational map. Then $\Phi$ is regular at all nonsingular points of $C$.

Proof. Let $p \in C$ be a nonsingular point. Since $\Phi$ is rational, by Definition 2.1.66 we may write $\Phi$ as $\Phi=\left(f_{0}: f_{1}: \cdots: f_{n}\right)$ where $f_{i} \in K(C)$ for $i=0,1, \ldots, n$. Let $t$ be a local parameter at $p$. Since $p$ is nonsingular, by Theorem 2.3.11, we may write $f_{i}=t^{e_{i}} u_{i}$ where $u_{i}(p) \neq 0$ for $i=0,1, \ldots, n$. Now let $e_{j}=\min \left\{e_{i}\right\}_{i=0}^{n}$. Then without changing $\Phi$ we may factor out $t^{e_{j}}$ to obtain

$$
\begin{aligned}
\Phi & =\left(f_{0}: \cdots: f_{n}\right) \\
& =\left(t^{e_{0}} u_{0}: \cdots: t^{e_{n}} u_{n}\right) \\
& =\left(t^{e_{0}-e_{j}} u_{0}: \cdots: t^{0} u_{j}: \cdots: t^{e_{n}-e_{j}} u_{n}\right)
\end{aligned}
$$


We notice that this rational map fails to be regular at $p$ (Definition 2.1.66) if and only if $\Phi(p)=0$ as $0 \notin \mathbb{P}^{n}$. We will show that this is not the case.

Evaluating this expression of $\Phi$ at $p$ we obtain

$$
\Phi(p)=\left(t^{e_{0}-e_{j}}(p) u_{0}(p): \cdots: u_{j}(p): \cdots: t^{e_{n}-e_{j}}(p) u_{n}(p)\right) \neq(0: \cdots: 0)
$$

since $u_{j}(p) \neq 0$. Thus $\Phi$ is regular at $p$. Since $p$ was an arbitrary nonsingular point, $\Phi$ is regular at all nonsingular points of $C$.

Corollary 2.4.6 Nonsingular birationally equivalent projective curves are isomorphic.

Proof. By Theorem 2.4.5, a rational map on a nonsingular projective curve is regular. Therefore a birational maps between nonsingular projective curves are regular. Hence, by Defintion 2.1.43, they are isomorphic.

Since all the properties of curves we care about are unique up to isomorphism we have the following definition.

Definition 2.4.7 Let $C$ be a plane projective curve. Then by Theorem 2.4.1 there exists a nonsingular projective curve $C^{\prime}$ birationally equivalent to $C$. Moreover, by Corollary 2.4.6, it is unique up to isomorphism. We call this curve the nonsingular projective model of $C$.

This relationship also works the other way around. Let $C^{\prime}$ be nonsingular projective curve. Then by Definition 2.1.70 of a curve, $C^{\prime}$ is birationally equivalent to a plane curve $C$.

In either direction, there exists a birational map $\Phi: C \rightarrow C^{\prime}$. Secondly, at all nonsingular points $U$ of $C$, by Theorem 2.4.5, $\Phi$ restricts to an isomorphism between $U$ and $\Phi(U) \subset C^{\prime}$. Thus, examining the local properties of nonsingular points of plane curves is equivalent to examining the local properties of the corresponding points on a nonsingular curve. 
By Theorem 2.1.75, we know that the function fields $K\left(C^{\prime}\right)$ and $K(C)$ are also isomorphic. So it is also sufficient to examine the function field of $K(C)$ instead of $K\left(C^{\prime}\right)$. Furthermore, the valuation rings at nonsingular points are invariant under isomorphism. Thus, examining the valuation rings of the function fields at nonsingular points of $C$ is equivalent to examining the corresponding points of $C^{\prime}$.

In the case of a curve defined by a polynomial $y^{2}-\varphi(x)$, we know that all the points are nonsingular (Remark 2.1.36). When we consider the projective closure $C$, then we know from Example 2.2.13 that only singularity is one point added, namely the point $(0: 1: 0)$. If we instead consider the nonsingular projective model $C^{\prime}$ of $C$ then by Theorem 2.4.5, there is an isomorphism $\Phi$ between $C \backslash(0: 1: 0)$ and its image in $\Phi(C)$. Since isomorphisms map open sets to open sets, and are one-to-one correspondences, we know that there are at most finitely many points of $C^{\prime}$ such that $\Phi^{-1}\left(C^{\prime}\right)=(0: 1: 0)$. However, we will see in the next chapter that there is only one such point. Thus, examining the local properties of $y^{2}-\varphi(x)$ is equivalent to examining the properties of all but one point of $C^{\prime}$. This is why we have focused on planar curves type and not more on arbitrary nonplanar curves.

Up to now, we have not defined valuations at singular points of a projective plane curve. To do so we need the following definitions. Recall from Theorem 2.3.9, that the valuation ring at a nonsingular point of a closed set is discrete if and only if $p$ is nonsingular. So in the same way as we did for plane curves (Definition 2.3.18), we define the valuation at a nonsingular point of an arbitrary affine curve.

Definition 2.4.8 Let $C$ be an affine curve. Let $p$ be a nonsingular point of $C$. Then by Theorem $2.3 .9, \mathcal{O}_{p}$ is a discrete valuation ring. Let $s(t)$ be a local parameter at $p$ as in Defintion 2.3.1. By Proposition 2.3.11, for all $z(t) \in K(C), z(t)=u(t) s(t)^{m}$ for some unit $u(t) \in \mathcal{O}_{p}$ and some integer $m$. We define the valuation at $p$ of $z(t)$ as the function $v_{p}: K(C) \rightarrow \mathbb{Z} \cup \infty$ given by

$$
v(z(t))=\left\{\begin{array}{lr}
\infty, & \text { if } z=0 \\
m, & \text { otherwise }
\end{array}\right.
$$


Clearly, this definition is in keeping with the definition given for plane curves (Definition 2.3.18). Generalizing Definition 2.3.24, the definition of the valuation at a projective point, we take the definition of the valuation at a nonsingular projective point $p$ of a closed set $A$ to be the valuation of $p \in A_{i}$ where $A_{i}$ is an affine curve isomorphic to the affine piece $U_{i}$ containing $p$ where $p$ is nonsingular.

We now give a more general definition of a valuation, in keeping with our previous definition, but also including the case of a singular point.

Definition 2.4.9 Let $C$ be a projective plane curve. Let $C^{\prime}$ be the nonsingular projective model of $C$ and let $\Phi$ be the birational map $\Phi: C^{\prime} \rightarrow C$. By Theorem 2.1.75, $K(C) \cong K\left(C^{\prime}\right)$, so let $\Phi^{*}$ be the isomorphism $\Phi^{*}: K(C) \rightarrow K\left(C^{\prime}\right)$. Let $p$ be a point of $C$. Let $S_{p} \subset C^{\prime}$ be the set of points such that

$$
S_{p}=\left\{p^{\prime} \in C^{\prime} \mid \Phi\left(p^{\prime}\right)=p\right\}
$$

We define the valuation at $p v_{p}$ as

$$
v_{p}(z)=\sum_{p^{\prime} \in S_{p}} v_{p^{\prime}}\left(\Phi^{*}(z)\right)
$$

Describing the valuations at nonsingular points of the projective model is one of the goals of the next chapter. However, in the case of the nonsingular projective model of $C_{A}$ defined by $y^{2}-\varphi(x)$, the results of the next section will allow us to explore the valuation at the singular point $p=(0: 1: 0)$ of the projective closure of $C_{A}$.

For projective plane curves $C$ we have shown that at each nonsingular point $p \in C$ there exists an open neighbourhood of $p$ isomorphic to an affine neighbourhood where the image of $p$ remains nonsingular (Lemma 2.3.23). We wish to now prove the same thing about arbitrary curves.

Theorem 2.4.10 Let $C$ be a projective curve and let $p \in C$ be nonsingular. Then there exists a neighbourhood $U \subset C$ of $p$ and an affine plane curve $A$ such that 
- there exists a rational map $\phi: C \rightarrow \bar{A}$ which provides a birational equivalence between $C$ and $\bar{A}$;

- there exists an open set $V \in A$ such that $\Phi_{U}: U \rightarrow V$ is an isomorphism and $\phi(p) \in V$;

- $\phi(p)$ is nonsingular.

Proof. By Definition 2.1.70, $C$ is birationally equivalent to a plane projective curve $C_{P}$. Let $p_{P}$ by the image of $p$. By Proposition 2.1.49, there exists a neighbourhood $U \subset C_{P}$ of $p_{P}$ isomorphic to an affine open neighbourhood $U_{B}$ of some affine plane curve $B$. Then by Theorem 2.4.10, there exists an affine plane curve $A$ birationally equivalent to $B$ where the image of $p_{P}$ is nonsingular. Combining all these birational maps gives a birational map $\phi: C \rightarrow \bar{A}$ where the image of $p$ is nonsingular. In addition, if we let $V$ be the open set of nonsingular points of $A$, then by Theorem 2.4.5, there exists an open subset $U \subset C$ of $p$ such that $\phi_{U}: U \rightarrow V$ is an isomorphism. Since $\phi(p) \in V$, and $p \in U$, we have the desired result.

This allows us to define valuations on nonsingular points of arbitrary projective curves.

Definition 2.4.11 Let $C$ be a projective curve and let $p \in C$ be nonsingular. Let $A$ be an affine plane curve where the birational map $\Phi^{-1}: C \rightarrow A$ is such that $\Phi^{-1}(p)=p_{A} \in A$ is nonsingular (Theorem 2.4.10). Let $\Phi^{*}$ be the isomorphism $\Phi^{*}: K(C) \rightarrow K(A)$ ('Theorem 2.1.75). We define the valuation at $p v_{p}$ as

$$
v_{p}(z)=v_{p_{A}}\left(\Phi^{*}(z)\right)
$$

This definition is well-defined. Indeed, given two affine plane curves $A$ and $B$ that fit the criterion of Definition 2.4.11, there exist two birational maps $\Phi_{A}: A \rightarrow C$ and $\Phi_{B}: C \rightarrow B$ whose composition $\Phi_{A} \circ \Phi_{B}$ gives a birational map from $A$ to $B$. Furthermore, if $p_{A} \in A$ is the nonsingular image of $\Phi_{A}^{-1}$, and $p_{B} \in B$ is the 
nonsingular image of $\Phi_{B}$, then by Theorem 2.4.5, $\Phi_{A} \circ \Phi_{B}$ restricts to an isomorphism between nonsingular neighbourhoods $U_{A}$ of $p_{A}$ and $U_{B}$ of $p_{B}$. Therefore, by Proposition 2.3.5, the valuation rings $\mathcal{O}_{p_{A}}$ and $\mathcal{O}_{p_{B}}$ are isomorphic. Consequently, $v_{p_{A}}\left(\Phi_{A}^{*}(z)\right)=v_{p_{B}}\left(\Phi_{B}^{*}(z)\right)$.

We may now define valuations at singular points of an arbitrary projective curve.

Definition 2.4.12 Let $C$ be a projective curve. Let $C^{\prime}$ be the nonsingular projective model of $C$ and let $\Phi$ be the birational map $\Phi: C^{\prime} \rightarrow C$. By Theorem 2.1.75, $K\left(C^{\prime}\right) \cong K(C)$, so let $\Phi^{*}$ be the isomorphism $\Phi^{*}: K(C) \rightarrow K\left(C^{\prime}\right)$. Let $p$ be a point of $C$. Let $S_{p} \subset C^{\prime}$ be the set of points such that

$$
S_{p}=\left\{p^{\prime} \in C^{\prime} \mid \Phi\left(p^{\prime}\right)=p\right\} .
$$

We define the valuation at $p v_{p}$ as

$$
v_{p}(z)=\sum_{p^{\prime} \in S_{p}} v_{p^{\prime}}\left(\Phi^{*}(z)\right) .
$$

A similar argument to that given for Definition 2.4 .9 shows that this definition is well-defined.

\subsection{Divisors of Points}

Now that we may use valuations to measure the zeros and poles of a rational function at a point, we introduce divisors; an object to record this information. This section is mainly focused on the case of projective plane curves. As per the results of the last section, this will still give us information about arbitrary curves. We begin with the definition of a divisor of an arbitrary curve.

Definition 2.5.1 Let $C$ be a projective curve. The additively written free abelian group which is generated by the points of $C$ is denoted by $\mathcal{D}_{C}$ and is called the divisor group of $C$. The elements of $\mathcal{D}_{C}$ are called divisors of $C$. 
A divisor may be written as a formal sum

$$
D:=\sum_{p \in C} n_{p} p \quad \text { with } \quad n_{p} \in \mathbb{Z}
$$

with all but finitely many $n_{p}=0$. Two divisors are added term-wise, i.e. for $D, D^{\prime} \in$ $\mathcal{D}_{C}$

$$
D+D^{\prime}=\sum_{p \in \mathcal{D}_{C}}\left(n_{p}+n_{p}^{\prime}\right) p
$$

Definition 2.5.2 The support of a divisor $D$, denoted $\operatorname{supp}(D)$, is defined as

$$
\operatorname{supp}(D):=\left\{p \in C \mid n_{p} \neq 0\right\}
$$

There exists a partial ordering on $\mathcal{D}_{C}$ which is defined by

$$
D \leq D^{\prime} \text { if and only if } n_{p} \leq n_{p}^{\prime} \quad \forall p \in C .
$$

This ordering is only partial. For example a divisor $D=p_{1}-p_{2}$ for some $p_{1}, p_{2} \in C$ is not comparable to the zero divisor as $n_{p_{1}}>0$ but $n_{p_{2}}<0$. When such a comparison is possible, a divisor $D$ is called positive (or effective) if $D \geq 0$.

Definition 2.5.3 The degree of a divisor, denoted $\operatorname{deg}(D)$, is defined by

$$
\operatorname{deg}(D):=\sum_{p \in C} n_{p}
$$

Definition 2.5.4 Let $C$ be a projective curve. The set of divisors of $C$ of degree 0 forms a normal subgroup $\mathcal{P}_{C} \subset \mathcal{D}_{C}$. We call $\mathcal{D}_{C} / \mathcal{P}_{C}$ the divisor class group of $C$. Two divisors in the same coset are called equivalent; we write $D_{1} \sim D_{2}$ for two equivalent divisors $D_{1}, D_{2} \in \mathcal{D}_{C}$.

From here we may construct divisors from functions on a projective curve $C$.

Proposition 2.5.5 ([16] p.153) Let $C$ be a projective plane curve. Let $z(T) \in K(C)$ 
be a nonzero function on $C$. Then $z(T)$ has finitely many zeros and poles.

Proof. Let $U_{1}, U_{2}$, and $U_{3}$ be an affine cover of $C$ as in Definition 2.1.21. Let $z_{1}(t)$, $z_{2}(t)$, and $z_{3}(t)$ be the dehomogenizations of $z(T)$ with respect to each cover. Let $S_{i}$ be the zero set of $z_{i}$ for $i=1,2,3$. Then from Example 2.1.7 we know that $S_{i}$ consists of finitely many points. Since the zero set of $z(T)$ is the union of the homogenizations of $S_{1}, S_{2}$ and $S_{3}$ it is finite. Repeating this argument for $1 / z(T)$ gives that $z(T)$ has finitely many poles.

This allows us to construct well-defined divisors from elements of $K(C)$.

Definition 2.5.6 Let $C$ be a plane curve (affine or projective). Let $0 \neq z \in K(C)$. Then the divisor of the function $z$ is denoted by $\operatorname{div}(z)$ and is defined as

$$
\operatorname{div}(z):=\sum_{p \in C} v_{p}(z) p
$$

A divisor $D$ is called principal if there exists a function $z \in K(C)$ such that $\operatorname{div}(z)=D$

A divisor provides a means to record the zeros and poles of a function. As such, we may use them to compare the zeros and poles of functions. We may even use them to define notions like regularity. To do so we need some futher results. The following theorem is one such result. We give the theorem without proof as it requires a lengthy analysis of exact sequences of maps on coordinate rings.

Theorem 2.5.7 ([4] p.112) Bézout's Theorem

Let $C$ be a projective plane curve defined by the homogeneous polynomial $c(X: Y: Z)$. Let $z(T) \in K[T]$. Then

$$
\sum_{p \in C} v_{p}(z)=\operatorname{deg}(c) \operatorname{deg}(z)
$$

Corollary 2.5.8 Let $C$ be a irreducible plane projective curve defined by the homogeneous polynomial $c(X: Y: Z)$. Let $z(X: Y: Z)=\frac{g(X: Y: Z)}{h(X: Y: Z)} \in K(C)$. Then

$$
\sum_{p \in C} v_{p}(z(X: Y: Z))=0
$$


Proof. From Bézout's Theorem we have that

$$
\begin{aligned}
\sum_{p \in C} v_{p}(z(X: Y: Z)) & =\sum_{p \in C}\left(v_{p}(g(X: Y: Z))-v_{p}(h(X: Y: Z))\right) \\
& =\operatorname{deg}(c) \operatorname{deg}(g)-\operatorname{deg}(c) \operatorname{deg}(h)
\end{aligned}
$$

However, since $z \in K(C)$ we have that $\operatorname{deg}(g)=\operatorname{deg}(h)$ and thus the desired result.

Corollary 2.5.9 Let $C$ be a projective curve and let $z(T) \in K(C)$. Then $\sum_{p \in C} v_{p}(z(T))=0$

Proof. Let $C^{\prime}$ be a nonsingular projective model of $C$ as in Theorem 2.4.1. By Definition 2.1.70 there exist a projective plane curve $C_{P}$ birationally equivalent to $C^{\prime}$. By transitivity, $C_{P}$ is also birationally equivalent to $C$. Let $\Phi$ be the birational map $\Phi: C^{\prime} \rightarrow C$. By Theorem 2.1.75, $K(C) \cong K\left(C^{\prime}\right)$, so let $\Phi^{*}$ be the isomorphism $\Phi^{*}: K(C) \rightarrow K\left(C^{\prime}\right)$. Let $p$ be a point of $C$. Let $S_{p} \subset C^{\prime}$ be the set of points such that

$$
S_{p}=\left\{p^{\prime} \in C^{\prime} \mid \Phi\left(p^{\prime}\right)=p\right\}
$$

By Definition 2.4.12,

$$
\left.\sum_{p \in C} v_{p}(z)\right)=\sum_{p \in C} \sum_{p^{\prime} \in S_{p}} v_{p^{\prime}}\left(\Phi^{*}(z)\right)=\sum_{p^{\prime} \in C^{\prime}} v_{p^{\prime}}\left(\Phi^{*}(z)\right)
$$

Let $\Phi_{2}$ be the birational map $\Phi: C_{P} \rightarrow C^{\prime}$. By Theorem 2.1.75, $K\left(C_{P}\right) \cong K\left(C^{\prime}\right)$, so let $\Phi_{2}^{*}$ be the isomorphism $\Phi_{2}^{*}: K\left(C^{\prime}\right) \rightarrow K\left(C_{P}\right)$. Let $p$ be a point of $C_{P}$. Let $R_{p} \subset C^{\prime}$ be the set of points such that

$$
R_{p}=\left\{p^{\prime} \in C^{\prime} \mid \Phi\left(p^{\prime}\right)=p\right\}
$$


Let $w=\Phi_{2}^{*}\left(\Phi^{*}(z)\right)$. Then, by Corollary 2.5.8 and Definition 2.4.11,

$$
0=\sum_{p \in C_{P}} v_{p}(w)=\sum_{p \in C_{P}} \sum_{p^{\prime} \in R_{p}} v_{p^{\prime}}\left(\Phi^{*}(z)\right)=\sum_{p^{\prime} \in C^{\prime}} v_{p^{\prime}}\left(\Phi^{*}(z)\right) .
$$

Combining this with Equation (2.7), we obtain that

$$
\sum_{p \in C} v_{p}(z(T))=\sum_{p^{\prime} \in C^{\prime}} v_{p}^{\prime}\left(\Phi^{*}(z)\right)=0
$$

as desired.

In the following example we show how to use Corollary 2.5.8 to find the valuations of functions at $p_{\infty}$.

Example 2.5.10 Let $C$ be the projective closure of the curve defined by the polynomial $y^{2}-\varphi(x)$ as in Remark 2.1.36. Then we know that the function $y \in K(C)$ is zero at all points $p_{i}=\left(0, a_{i}\right)$ where $\varphi\left(a_{i}\right)=0$ and only at these points. From Example 2.3.14, we also know that $y$ is a local parameter at each of these points. Thus $v_{p_{i}}(y)=1$. We have not yet seen the valuation of $y$ at $p_{\infty}=(0: 1: 0)$. We may calculate this using Bézout's Theorem.

$$
\begin{aligned}
0 & =\sum_{p \in C} v_{p}(y)=\sum_{i=1}^{\operatorname{deg}(\varphi(x))} v_{p_{i}}(y)+v_{p_{\infty}}(y), \\
v_{p_{\infty}}(y) & =-\sum_{i=1}^{\operatorname{deg}(\varphi(x))} 1=-\operatorname{deg}(\varphi(x)) .
\end{aligned}
$$

As such the divisor of $y$ is

$$
\operatorname{div}(y)=\sum_{i=1}^{\operatorname{deg}(\varphi(x))} p_{i}-(\operatorname{deg}(\varphi(x))) p_{\infty} .
$$

Now, consider the function $x \in K(C)$. We know that if $x$ divides $\varphi(x)$ then $p_{0}=$ $(0,0) \in C$ is the only zero of $x$ and $v_{p}(x)=2$ (Example 2.3.14). If $x$ does not divide 
$\varphi(x)$ then $\varphi(0)=a \neq 0$ where $a \in K$. Thus there are two points on $C$ where $x=0$, corresponding to the two roots of $y^{2}-a$, which we will denote as $y_{1}$ and $y_{2}$. The two points are $p_{1}=\left(0, y_{1}\right)$ and $p_{2}=\left(0, y_{2}\right)$. Since $x=0$ is not tangent to these points, from Proposition 2.3.12, $v_{p_{1}}(x)=v_{p_{2}}(x)=1$. In either case, exploring the valuations of $x$, we have that

$$
\begin{aligned}
0 & =\sum_{p \in C} v_{p}(x)=2+v_{p_{\infty}}(x), \\
v_{p_{\infty}}(x) & =-2 .
\end{aligned}
$$

As such, the divisor of $x$ is

$$
\operatorname{div}(x)=\left\{\begin{array}{lr}
2 p_{0}-2 p_{\infty}, & \text { if } \varphi(0)=0 \\
p_{1}+p_{2}-2 p_{\infty}, & \text { otherwise }
\end{array}\right.
$$

Example 2.5.11 Let $C$ be the projective closure of the curve defined by the polynomial $y^{2}-\varphi(x)$. Then we know from the above example that $v_{p_{\infty}}(y)=-\operatorname{deg}(\varphi(x))=$ $-(2 m+1)$ for some $0 \leq m \in \mathbb{Z}$ (the degree of $\varphi(x)$ is assumed to be odd as in Example 2.1.62). We also know that $v_{p_{\infty}}(x)=-2$. Using this information we may find a local parameter at $p_{\infty}$. Indeed,

$$
v_{p_{\infty}}\left(\frac{x^{m}}{y}\right)=v_{p_{\infty}}\left(x^{m}\right)-v_{p_{\infty}}(y)=-2 m-(-2 m-1)=1
$$

and thus $\frac{x^{m}}{y}$ a local parameter at $p_{\infty}$.

Proposition 2.5.12 Let $C$ be plane curve (affine of projective). A function $z \in K(C)$ is regular if and only if $\operatorname{div}(z) \geq 0$.

Proof. A function $z \in K(C)$ is regular if and only if it is regular at every point $p \in C$ (Definition 2.1.37). By Defintion 2.3.1 a function $z$ is regular at a point $p$ if and only if $z \in \mathcal{O}_{p}$. In addition, $z \in \mathcal{O}_{p}$ if and only if $v_{p}(z) \geq 0$ by Theorem 3.2.3. Since this is true for all $p \in C, \operatorname{div}(p) \geq 0$ by Definition 2.5 .6 


\subsection{Differential Forms}

In this section we consider maps which take functions on a closed set to functions on tangent spaces. We build up to the definition of a regular differential form by defining various maps from rational functions to the duals of tangent spaces. Using regular differential forms on open sets, we define rational differential forms. A rational differential form is a function and so using Sections 3.2 and 2.5 we may assign divisors to them.

As differential forms rely on tangent spaces, we revisit some of the ideas put forth in Section 2.2. Recall that given a projective plane curve $C$ and a point $p \in C$, there exists an affine piece of $C, U$ say, such that $p \in U$ (Definition 2.1.21). Moreover, by Lemma $2.1 .71 K(C) \cong K(U)$. We have used this result repeatedly in the last two sections.

We also saw in Example 2.2.11 that a tangent line at a projective point $p$ is exactly the homogenization of the tangent line of $p$ on some affine closed set $A$ isomorphic to $U$ (typically $A$ is the dehomogenization of that piece). This implies that the tangent space is a local property, i.e. we need only consider affine neighbourhoods to find the tangent space of a projective point. This is why it again suffices to focus mainly on affine plane curves.

Let $C$ be a plane affine curve defined by the polynomial $c(x, y)$ and let $p=\left(x_{0}, y_{0}\right)$ be a point on $C$. We noted in Remark 2.2.6 that any polynomial function $g(x, y) \in$ $K[C]$ may be expressed in the form.

$$
g(x, y)=g_{0}\left(x-x_{0}, y-y_{0}\right)+g_{1}\left(x-x_{0}, y-y_{0}\right)+\cdots+g_{n}\left(x-x_{0}, y-y_{0}\right)
$$

Where $g_{i}$ are homogeneous polynomials in $x-x_{0}$ and $y-y_{0}$ of degree $i$. Writing $c(x, y)$ in this form yields

$$
c(x, y)=c_{0}\left(x-x_{0}, y-y_{0}\right)+c_{1}\left(x-x_{0}, y-y_{0}\right)+\cdots+c_{n}\left(x-x_{0}, y-y_{0}\right) .
$$


Note that the linear form $c_{1}\left(x-x_{0}, y-y_{0}\right)$ from Equation (2.9) corresponds to a polynomial function in $K\left[\mathbb{A}^{2}\right]$. However, we are interested in functions on the tangent space $\Theta_{p}$ of $p$ as defined in Definition 2.2.9. We will achieve this with the following definition.

Definition 2.6.1 Let $C$ be a plane affine curve and let $p=\left(x_{0}, y_{0}\right)$ be a point on $C$. Let $g(x, y) \in K[C]$. Then $g(x, y)$ may be expressed in the form

$$
g(x, y)=g_{0}\left(x-x_{0}, y-y_{0}\right)+g_{1}\left(x-x_{0}, y-y_{0}\right)+\cdots+g_{n}\left(x-x_{0}, y-y_{0}\right),
$$

as in Equation 2.8. We now restrict the linear term to a function on $\Theta_{p}$, i.e. set $d_{p}(g)=\left.g_{1}\left(x-x_{0}, y-y_{0}\right)\right|_{\Theta_{p}}$. Then $d_{p}(g): \Theta_{p} \rightarrow K$ is called the differential of $g$ at $p$.

Example 2.6.2 Let $C$ be a plane affine curve defined by the polynomial $c(x, y)=$ $y^{2}-x^{3}+x$ and let $p=\left(x_{0}, y_{0}\right)$ be a point on $C$. We begin by describing the tangent space $\Theta_{p}$ of $C$ at $p$. By Definition 2.2.9, $\Theta_{p}$ is the closed set defined by the polynomial

$$
\begin{aligned}
l(x, y) & =\frac{\partial c}{\partial x}(p) x+\frac{\partial c}{\partial y}(p) y \\
& =\left(-3 x_{0}^{2}+1\right) x+2 y_{0} y
\end{aligned}
$$

Let $x-y=g(x, y) \in K[C]$. Then $g(x, y)$ may be written as

$$
g(x, y)=x_{0}-y_{0}+\left(x-x_{0}\right)-\left(y-y_{0}\right)
$$

Then by Definition 2.6.1, $d_{p}(g)=\left(x-x_{0}\right)-\left(y-y_{0}\right)_{\mid \Theta_{P}}$. Now using $l(x, y)=0$ on $\Theta_{p}$ we can write $d_{p}(g)$ as

$$
\begin{aligned}
d_{p}(g) & =\left(x-x_{0}\right)-\left(y-y_{0}\right)_{\mid \Theta_{P}} \\
& =x-x_{0}+y_{0}+\frac{-3 x_{0}^{2}+1}{2 y_{0}} x
\end{aligned}
$$

Again notice that $d_{p}(g)$ is a function. 
It follows from Definition 2.6.1 of a differential, that for all $g(x, y), h(x, y) \in K[C]$ we have

$$
\begin{aligned}
d_{p}(g+h) & =d_{p}(g)+d_{p}(h) \\
d_{p}(g h) & =g(p) d_{p}(h)+h(p) d_{p}(g)
\end{aligned}
$$

which are again maps from $\Theta_{p}$ to $K$.

Remark 2.6.3 In light of the above, we see that the set differentials $d_{p}(g)$ for all $g(x, y) \in K[C]$ form a vector space over $K$.

Proposition 2.6.4 Let $C$ be an affine curve with $p=\left(x_{0}, y_{0}\right) \in C$ a nonsingular point. Let $g(x, y) \in K[C]$. Then $d_{p}(g)$ is a linear function on $\Theta_{p}$.

Proof. Without loss of generality, as translations are isomorphisms, we can assume that $p=(0,0)$. Then $d_{p}(g)=g_{1}(x)+g_{2}(y)$ for some $g_{1}, g_{2} \in K$. Since $p$ is nonsingular, we saw in Example 2.2.7 that there is a unique tangent line $\Theta_{p}$ to $C$ at $p$. Parameterizing $\Theta_{p}$ with the variable $s$ we have that

$$
\Theta_{p}=\{(x, y) \mid x=a s, y=b s\}
$$

where $a=\frac{\partial c}{\partial x}(p)$ and $b=\frac{\partial c}{\partial y}(p)$. Now checking $d_{p}(g(x, y))$ on $\Theta_{p}$ we have

$$
d_{p}(g)_{\mid \Theta_{p}}=g_{1}(a s)+g_{2}(b s)=\left(g_{1} a+g_{2} b\right) s
$$

which is clearly linear in $s$ and hence on $\Theta_{p}$.

We now define a vector space of which $d_{p}(g)$ is an element.

Definition 2.6.5 Let $C$ be an affine plane curve and let $p$ be a point on $C$. The vector space of linear forms on the tangent space $\Theta_{p}$ is called the dual of the tangent space $\Theta_{p}$ and is denoted $\Theta_{p}^{*}$. 
By Remark 2.6.3 and Proposition 2.6.4, we see that the vector space of differentials at $p$ is a subspace of $\Theta_{p}^{*}$.

\subsubsection{Regular Differential Forms}

Given a regular function $z(t) \in K[C]$ for an affine plane curve $C$, the differential $d_{p}(z(t))$ is a map, dependent on the point $p$, mapping to the tangent space of $p$. In this section we discuss something more global. Given a regular function $z(t)$ we wish to define a map from points $p$ to a linear function on the tangent space to $p$. We first define all such maps.

Let $C$ be an affine plane curve. Let $\Phi[C]$ be the set of all possible functions $\phi$ sending each point $p \in C$ to a linear form in $\Theta_{p}^{*}$. If we set $(g \phi)(p)=g(p) \phi(p)$ for all $g(x, y) \in K[C]$ then we have that $\Phi[C]$ is a $K[C]$-module.

Definition 2.6.6 Let $C$ be an affine curve and let $g(t) \in K[C]$. Then $g(t)$ defines an element $\phi_{g} \in \Phi[C]$ by $\phi_{g}(p)=d_{p}(g(t))$. We denote this function $\phi$ by $d(g(t))$ and call it the differential form of $g(t)$.

Example 2.6.7 Let $C$ be an affine plane curve and let $g(x, y) \in K[C]$ be such that $g(x, y)=x^{2}$. Then $d g=d\left(x^{2}\right)$ is an element of $\Phi[C]$, i.e. $d\left(x^{2}\right)$ is a map on the points $p=\left(x_{0}, y_{0}\right) \in C$ such that $d\left(x^{2}\right)(p) \in \Theta_{p}^{*}$. More precisely,

$$
d\left(x^{2}\right)(p)=d_{p}\left(x^{2}\right)=\left.\left(\frac{\partial x^{2}}{\partial x}(p) x+\frac{\partial x^{2}}{\partial y}(p) y\right)\right|_{\Theta_{p}}=\left.2 x(p) x\right|_{\Theta_{p}}=\left.2 x_{0} x\right|_{\Theta_{p}} .
$$

Notice that at all points $p=\left(0, y_{0}\right)$ we have that $d\left(x^{2}\right)(p)$ is the zero function on $\Theta_{p}$. Notice also that if $p$ is nonsingular with a tangent line defined by $x=0$ then $d\left(x^{2}\right)(p)$ is also the zero function on $\Theta_{p}$. Indeed $\left.2 x_{0} x\right|_{\Theta_{p}}=\left.2 x_{0} 0\right|_{\Theta_{p}}=\left.0\right|_{\Theta_{p}}$. We will revisit this idea a little later on.

To each function $g(x, y) \in K(C)$ we have defined a differential form $d(g(x, y))$. For this reason, it makes sense to consider a function that takes $g(x, y)$ to $d(g(x, y))$. 
Proposition 2.6.8 Let $C$ be an affine plane curve. Define the function $d: K[C] \rightarrow$ $\Phi[C]$ by $d(g(x, y))=d(g(x, y))$ for all $g \in K[C]$. It has the following properties.

1. $d(g(x, y)+h(x, y))=d(g(x, y))+d(h(x, y))$

2. $d(g(x, y) h(x, y))=g(x, y) \cdot d(h(x, y))+h(x, y) \cdot d(g(x, y))$

3. $d\left(g\left(h_{1}(x, y), h_{2}(x, y)\right)\right)$

$$
=\frac{\partial g}{\partial x}\left(h_{1}(x, y), h_{2}(x, y)\right) \cdot d\left(h_{1}(x, y)\right)+\frac{\partial g}{\partial y}\left(h_{1}(x, y), h_{2}(x, y)\right) \cdot d\left(h_{2}(x, y)\right)
$$

for all $g(x, y), h(x, y) \in K[C]$.

Proof. The first two properties are a direct result of the properties of $d_{p}$ function given in Equations (2.10) and (2.11). To prove the third property, suppose that $p \in C$. Then

$$
\begin{aligned}
& d\left(g\left(h_{1}(x, y), h_{2}(x, y)\right)\right)(p) \\
= & d_{p}\left(g\left(h_{1}(x, y), h_{2}(x, y)\right)\right) \\
= & \frac{\partial}{\partial x} g\left(h_{1}(x, y), h_{2}(x, y)\right)(p) x+\frac{\partial}{\partial y} g\left(h_{1}(x, y), h_{2}(x, y)\right)(p) y \\
= & \left(\frac{\partial g}{\partial x}\left(h_{1}(p), h_{2}(p)\right) \cdot \frac{\partial h_{1}}{\partial x}(p)+\frac{\partial g}{\partial y}\left(h_{1}(p), h_{2}(p)\right) \frac{\partial h_{2}}{\partial x}(p)\right) x \\
& +\left(\frac{\partial g}{\partial x}\left(h_{1}(p), h_{2}(p)\right) \cdot \frac{\partial h_{1}}{\partial y}(p)+\frac{\partial g}{\partial y}\left(h_{1}(p), h_{2}(p)\right) \frac{\partial h_{2}}{\partial y}(p)\right) y \\
= & \frac{\partial g}{\partial x}\left(h_{1}(p), h_{2}(p)\right) \cdot d h_{1}(p)+\frac{\partial g}{\partial y}\left(h_{1}(p), h_{2}(p)\right) \cdot d h_{2}(p) .
\end{aligned}
$$

Since this is true for all $p$, we have the desired result.

Using the properties of Proposition 2.6.8, we may express a differential form in various ways.

Example 2.6.9 Let $C$ be an affine plane curve defined by the polynomial $c(x, y)=$ $y^{2}-\varphi(x)$. On $C$, we have that $y^{2}=\varphi(x)$ and taking $d$ of both sides we obtain that

$$
2 y \cdot d y=\varphi^{\prime}(x) d x .
$$


Hence the differential form $d x$ may also be expressed as $\frac{2 y}{\varphi^{\prime}(x)} d y$.

Recall that $\Phi[C]$ is the set of all possible functions $\phi$ sending each point $p \in C$ to a linear form in $\Theta_{p}^{*}$ and that $\Phi[C]$ is a $K[C]$-module. For any open subset $U \subset C$ we define $\Phi[U]$ as the set of all possible functions $\phi$ sending each point $p \in U$ to a linear form in $\Theta_{p}^{*}$. If we again set $(g \phi)(p)=g(p) \phi(p)$ for all $g(x, y) \in K[U]$ then we have that $\Phi[U]$ is a $K[U]$-module.

With this in mind we give the following definition.

Definition 2.6.10 ([16] p.195) Let $C$ be an affine curve. An element $\phi \in \Phi[C]$ is a regular differential form on $C$ if every point $p$ has a neighbourhood $U$ such that the restriction of $\phi$ to $U$ belongs to the $K[U]$-submodule of $\Phi[U]$ generated by the elements $d(g(T))$ with $g(T) \in K[U]$.

All regular differential forms on $C$ form a $K[C]$-module called the module of regular differential forms ([16] p.195). It shall be denoted by $\Omega[C]$.

For all open subsets $U \subset C$ and for all $g(t) \in K[C], g(t)_{\mid U} \in K[U]$. Thus $d(g(t)) \in \Omega[C]$ by Definition 2.6.10. So we may refine our definition of the map $d$. The image space of $d$ is contained in $\Omega[C] \subset \Phi[C]$. As such, $d: K[C] \rightarrow \Omega[C]$ is a more accurate definition.

By Definition 2.6.10, any regular differential form $\phi$ may be written in the form

$$
\phi=\sum_{i=1}^{m} h_{i}(t) d\left(g_{i}(t)\right)
$$

whenever restricted to an affine neighbourhood $U$ of any point $p \in C$, where the $h_{i}(t), g_{i}(t) \in K[U]$. This means that to show a differential form is regular we need to find such expressions at every point.

Example 2.6.11 Let $C$ be an affine plane curve defined by the polynomial $y^{2}-\varphi(x)$. Consider the differential form $\omega=\frac{d x}{y}$. For any point $p=\left(p_{1}, p_{2}\right) \in C$, with $p_{2} \neq 0$, $\frac{d x}{y}$ is certainly regular. At all such points we may take $U$ to be $C \backslash\{y=0\}$ and represent $\omega$ as $\frac{d x}{y} \in \Omega[U]$. 
At points $p$ where $p_{2}=0$, using equation (2.13) from the previous example, we may rewrite our differential form as $\frac{2}{\varphi^{\prime}(x)} d y$. Since $C$ is nonsingular and $p=\left(p_{1}, 0\right) \in C$, $\varphi\left(p_{1}\right)=0$ but $\varphi^{\prime}\left(p_{1}\right) \neq 0$. At all such points we may take $U$ to be $C \backslash\left\{\varphi^{\prime}(p)=0\right\}$ and represent $\omega$ as $\frac{2}{\varphi^{\prime}(x)} d y \in \Omega[U]$.

Hence, at all points $p$ there exists a neighbourhood $U$ such that $\omega \in \Omega[U]$. So $\omega$ is a regular differential form of $C$, i.e. $\omega \in \Omega[C]$.

We may view the function $d$ as a homomorphism of additive abelian groups and thus investigate the kernel of the map $d: K[C] \rightarrow \Omega[C]$.

Example 2.6.12 Let $C$ be an affine plane curve. Suppose that $g(x, y) \in K[C]$. Then

$$
\begin{aligned}
d(g)=0 & \Leftrightarrow d_{p}(g)=0 \quad \forall p \in C \\
& \left.\Leftrightarrow\left(\frac{\partial g}{\partial x}(p) x+\frac{\partial g}{\partial y}(p) y\right)\right|_{\Theta_{p}}=0 \quad \forall p \in C \\
& \Leftrightarrow \frac{\partial g}{\partial x}(p)=\frac{\partial g}{\partial y}(p)=0 \quad \forall p \in C \quad \text { or } \quad g(C)=0 \\
& \Leftrightarrow g(x, y) \in K \quad \text { or } \quad g(C)=0 .
\end{aligned}
$$

Thus $d(g(x, y))=0$ if and only if $g(x, y)$ is constant or identically zero on $C$.

We will now define the regular differential forms of a projective plane curve. The definition used to define a regular differential form for affine plane curves may easily be adapted to projective plane curves.

Definition 2.6.13 ([16] p.195) Let $C$ be an projective curve. An element $\phi \in \Phi[C]$ is a regular differential form on $C$ if every point $p$ has neighbourhood $U$ isomorphic to an affine neighbourhood $A$, such that the restriction of $\phi$ to $U$ is isomorphic to an element of the $K[A]$-submodule of $\Phi[A]$ generated by the elements $d(g(T))$ with $g(T) \in K[A]$.

This definition is of the same flavour as the one used to define a valuation on a projective point in Definition 2.3.24. In both cases, the definitions rely on projective 
neighbourhoods which are isomorphic to affine neighbourhoods. In addition, when we are referring to a nonsingular projective point $p$, Lemma 2.3.23 allows us to assume without loss of generality that the image of $p$ in the affine neighbourhood is nonsingular. So from now on we will drop any mention of the isomorphism and just refer to affine neighbourhoods of projective points; the isomorphism being tacitly implied.

Again, by Definition 2.6.13, any regular differential form $\phi$ may be written in the form

$$
\phi=\sum_{i=1}^{m} h_{i}(t) d\left(g_{i}(t)\right)
$$

whenever restricted to a affine neighbourhood $U$ of any point $p \in C$, where the $h_{i}(t), g_{i}(t) \in K[U]$.

Theorem 2.6.14 ([16] p.197) Let $C$ be a projective curve. Any nonsingular point $p \in C$ has an affine neighbourhood $U$ such that $\Omega[U]$ is a free $K[U]$-module of rank 1 .

Proof. Recall from Theorem 2.4.10 that every point $p$ has an affine neighbourhood where the image of $p$ is nonsingular. So, without loss of generality, it suffices to consider neighbourhoods of nonsingular points of affine plane curves.

Let $C$ be an affine plane curve defined by the polynomial $c(x, y)$. Since $c(x, y)$ defines $C$, it is zero on $C$. Taking $d$, we have

$$
0=d(c(x, y))=\frac{\partial c}{\partial x} d x+\frac{\partial c}{\partial y} d y
$$

Since $p$ is nonsingular, at least one of the partial derivatives is non zero at $p$. Without loss of generality assume that $\frac{\partial c}{\partial x}(p) \neq 0$. Let $U$ be the open set $C \backslash\left\{\frac{\partial c}{\partial x}=0\right\}$. Then rearranging Equation (2.16), gives us that

$$
d x_{\mid U}=-\frac{\frac{\partial c}{\partial y}}{\frac{\partial c}{\partial x}} d y_{\mid U}
$$

Notice that on the neighbourhood $U$ of $p$ the function $-\frac{\frac{\partial c}{\partial y}}{\frac{\partial c}{\partial x}}$ is regular. Now as in 
Equation 2.14, for any $d g \in \Omega[U]$,

$$
d(g(x, y))=\sum_{i=1}^{m} g_{i}(x, y) d\left(h_{i}(x, y)\right) \quad \text { where } \quad g_{i}(x, y), h_{i}(x, y) \in K[U] .
$$

By the chain rule $d\left(h_{i}(x, y)\right)$ may be written in the form $d\left(h_{i}(x, y)\right)=\frac{\partial h_{i}}{\partial x}(x, y) d x+$ $\frac{\partial h_{i}}{\partial y}(x, y) d y$ where $\frac{\partial h_{i}}{\partial x}(x, y), \frac{\partial h_{i}}{\partial y}(x, y) \in K[U]$. Our goal now is to write $d\left(h_{i}(x, y)\right)$ in terms of $d x$ or $d y$ alone. On $U$, substituting in Equation (2.17) into the expression for $d(g(x, y))$ given in (2.18), we may write $d(g(x, y))_{\mid U}$ as

$$
d(g(x, y))_{\mid U}=\sum_{i=1}^{m} g_{i}(x, y)\left(\frac{\partial h_{i}}{\partial x}(x, y)\left(-\frac{\frac{\partial c(x, y)}{\partial y}}{\frac{\partial c(\boldsymbol{x}, y)}{\partial x}}\right)+\frac{\partial h_{i}}{\partial y}(x, y)\right) d y .
$$

Thus every element of $\Omega[U]$ may be written as $h(x, y) d y$ for some $h(x, y) \in K[U]$, and hence $\Omega[U]$ is a rank 1 module.

Corollary 2.6.15 ([16] p.198) Let $C$ be a projective curve. If $s(T)$ is a local parameter at a nonsingular point $p$ in a neighbourhood $U$, then $d(s(T))$ generates $\Omega[U]$ as a $K[U]$-module for some affine neighbourhood $U$ of $p$.

Proof. Again from Theorem 2.4.10, every point $p$ has an affine neighbourhood where the image of $p$ is nonsingular. So, without loss of generality, it suffices to consider neighbourhoods of nonsingular points of affine plane curves.

Suppose that $s(x, y)$ is a local parameter at $p \in C$. Then $s(x, y)$ is regular at $p$ and hence regular in some neighbourhood $U_{1}$ of $p$. By Theorem 2.6.14, there exists a function $z(x, y) \in K\left[U_{2}\right]$ for some neighbourhood of $U_{2}$ of $p$ such that $d(z(x, y))$ is a basis for $\Omega\left[U_{2}\right]$. Hence, on the neighbourhood $U_{1} \cap U_{2}, d(z(x, y))$ also forms a basis of $\Omega\left[U_{1} \cap U_{2}\right]$ and so there exists a function $g(x, y) \in K\left[U_{1} \cap U_{2}\right]$ such that $d(s(x, y))=g(x, y) d(z(x, y))$ on $U_{\mathbf{1}} \cap U_{2}$.

Since $g(x, y)$ is regular and non-zero at $p$ there exists a neighbourhood of $p$, say $U \subset U_{1} \cap U_{2}$ such that $1 / g(x, y)$ is regular on $U$. Thus $d(z(x, y))=$ $(1 / g(x, y)) d(s(x, y))$ is regular on $U$. As $d(z(x, y))$ is a basis of $\Omega[U]$, for all 
$\omega \in \Omega[U]$, there exists an $h(x, y) \in K[U]$ such that $\omega=h(x, y) d(z(x, y))=$ $(h(x, y) / g(x, y)) d(s(x, y)) \in \Omega[U]$. Therefore $d(s(x, y))$ is a basis of $\Omega[U]$.

Using Corollary 2.6.15, we may say what it means for a differential form to be zero at a point. We begin with the following proposition.

Proposition 2.6.16 Let $C$ be an affine plane curve and let $s(x, y) \in \mathcal{O}_{p}$ be a local parameter at a nonsingular point $p$. Then $d(s)(p) \neq 0_{\mid \Theta_{p}}$.

Proof. Since $p$ is nonsingular, from Proposition 2.3.12, all the defining polynomials of lines not tangent to $C$ through $p$ are also local parameters. Let $l(x, y)$ define one such line. Since $l(x, y)$ and $s(x, y)$ are local parameters, by Proposition 2.3.13, $s(x, y)=u(x, y) l(x, y)$ for some unit $u(x, y) \in \mathcal{O}_{p}$. Then evaluating $d(s(x, y))$ at $p$ we have

$$
\begin{aligned}
d(s(x, y))(p) & =d(u(x, y) l(x, y))(p) \\
& =\frac{\partial u(x, y) l(x, y)}{\partial x}(p) x+\frac{\partial u(x, y) l(x, y)}{\partial y}(p) y \\
& =\left(u(p) \frac{\partial l}{\partial x}(p)+l(p) \frac{\partial u}{\partial x}(p)\right) x+\left(u(p) \frac{\partial l}{\partial y}(p)+l(p) \frac{\partial u}{\partial y}(p)\right) y
\end{aligned}
$$

Since $l(p)=0$, we have

$$
\begin{aligned}
d(s(x, y))(p) & =\left(u(p) \frac{\partial l}{\partial x}(p)\right) x+\left(u(p) \frac{\partial l}{\partial y}(p)\right) y \\
& =u(p)\left(\frac{\partial l}{\partial x}(p) x+\frac{\partial l}{\partial y}(p) y\right) \\
& =u(p) l(x, y) .
\end{aligned}
$$

Let $l_{p}(x, y)$ denote the tangent line to $C$ at $p$. Since $l(x, y)$ is not tangent to $c(x, y)$ at $p, l(x, y)$ is not a multiple of $l_{p}(x, y)$. Therefore, by Lemma 2.1.6, $l(x, y)$ and $l_{p}(x, y)$ have only finitely many common zeros and so $l(x, y)$ is not the 0 function on $\Theta_{p}$. Since $u$ is a unit in $\mathcal{O}_{p}$ it is not zero at $p$ by Remark 2.3.3. Therefore $d(s(x, y))(p) \neq 0_{\mid \Theta_{p}}$. 
Example 2.6.17 Let $C$ be an affine plane curve defined by the polynomial $y^{2}-\varphi(x)$ as in Remark 2.1.36. Consider the regular differential $d y \in \Omega[C]$. From Example 2.3.14 we know that at all points $p=\left(x_{0}, 0\right) \in C, y$ is a local parameter and hence $d y(p) \neq 0$. Furthermore, from Example 2.3.14, we know that $x-x_{0} \in K[C]$ is a local parameter at $p$. Using the results of Proposition 2.6.8, we notice that

$$
d\left(x-x_{0}\right)=d x-d x_{0}=d x-0=d x .
$$

Second, on all points $p=\left(x_{0}, y_{0}\right) \in C$, where $y_{0} \neq 0$, we have that

$$
d y=\frac{\varphi^{\prime}(x)}{2 y} d x
$$

Combining these results, we have that

$$
d y=\frac{\varphi^{\prime}(x)}{2 y} d\left(x-x_{0}\right)
$$

Hence $d y=0$ at the points where $\varphi^{\prime}\left(x_{0}\right)=0$. Intuitively, these are the points where the tangent line to $C$ is horizontal.

We make the following remark which allows us to define the zeros of a regular differential form.

Remark 2.6.18 Let $C$ be a projective curve and let $p$ be a nonsingular point of $C$. By Corollary 2.6.15, for all $\omega \in \Omega[C]$, there exists an affine neighbourhood $U$ of $p$ upon which $\omega=z(x, y) d(s(x, y))$ for some $z(x, y) \in K[U]$, where $s(x, y)$ is a local parameter at $p$. Therefore $\omega(p)=0$ if and only if $z(p) d(s(x, y))(p)=0$. However, by Proposition 2.6.16, $d(s(x, y))(p) \neq 0$. Therefore $\omega(p)=0$ if and only if $z(p)=0$.

Lemma 2.6.19 ([16] p.202) Let $C$ be a projective curve. The set of points on $C$ at which a regular differential form is 0 is closed.

Proof. Dehomogenizing our curve in each variable creates an affine cover. In each affine piece, consider an affine neighbourhood $U$ of any point $p$ of $C$. Namely we may 
consider the neighbourhood of Corollary 2.6.15, where we may express the differential form as $\omega=g(t) d(s(t))$ where $s(t)$ is the local parameter at $p$. By Remark 2.6.18, the statement that $\omega=0$ is equivalent to $g(t)=0$ which defines a closed set. Now, as the open set $U$ may be viewed as an open subset of an affine plane curve, its complement consists of finitely many points (Example 2.1.7). Thus, we only need to express $\omega$ on finitely many neighbourhoods $U_{i}$, and taking the union of the closed sets defined by $\omega_{\mid U_{i}}=0$ defines a closed set on each affine piece, and their union defines a closed set on $C$.

\subsubsection{Rational Differential Forms and their Divisors}

By pasting regular differential forms on open sets we may define a rational differential form. A rational differential form is an equivalence class of regular functions and so using Sections 3.2 and 2.5 we will assign divisors to them. We then show that all rational differential forms are in the same divisor class. We will see that regular differential forms may be identified as a subset of the rational ones. By using divisors, we may then determine which rational differential forms are regular. We begin by constructing the equivalence relation to be used in the definition of a rational differential form.

Proposition 2.6.20 ([16] p.202) Let $C$ be a projective curve and let $U$ and $U^{\prime}$ be open subsets of $C$. Let $\omega \in \Omega[U]$ and $\omega^{\prime} \in \Omega\left[U^{\prime}\right]$. We define a relation on the pairs of regular differential forms and open sets as follows. We write that $(\omega, U) \sim\left(\omega^{\prime}, U^{\prime}\right)$ if $\omega=\omega^{\prime}$ on $U \cap U^{\prime}$. This forms an equivalence relation.

Proof. The relation is certainly reflexive and symmetric. To prove that its transitive, suppose that $\left(a, U_{a}\right) \sim\left(b, U_{b}\right)$ and $\left(b, U_{b}\right) \sim\left(c, U_{c}\right)$. Then by definition $a=b$ on $U_{a} \cap U_{b}$ and $b=c$ on $U_{b} \cap U_{c}$. Then $a=c$ on $U_{a} \cap U_{b} \cap U_{c}$. Since this is a finite intersection of open sets, it is also open. Furthermore, since $a-c=0$ on an open subset of $U_{a} \cap U_{c}, a-c=0$ on $U_{a} \cap U_{c}$ by Corollary 2.1.56. Therefore $\left(a, U_{a}\right) \sim$ $\left(c, U_{c}\right)$. 
Definition 2.6.21 ([16] p.202) An equivalence class under the equivalence relation defined in Proposition 2.6.20 is called a rational differential form on $C$. The set of rational differential forms is denoted by $\Omega(C)$.

We routinely abuse notation by identifying a rational differential form with a representative of its equivalence class.

Example 2.6.22 Let $C$ be the projective closure of the affine plane curve $A$ defined by the polynomial $y^{2}-\varphi(x)$. We know from Definition 2.1.21 that $A$ may be viewed as an open subset of $C$. Consider the differential form $(1 / y) d y$ on $A$. At all points $p=\left(x_{0}, 0\right)$, from Example 2.3.14, we know that $y$ is a local parameter and thus $((1 / y) d y)(p)=(1 / y)(p) d y(p)$ which is not defined at $p$. However, if we consider the open set $U \subset A$ defined by $U=\left\{p=\left(x_{0}, y_{0}\right) \in C \mid y_{0} \neq 0\right\}$. Then on $U$ we know from Example 2.6.17, that $d y=\frac{\varphi^{\prime}(x)}{y} d x$ is regular. Therefore on $U,(1 / y) d y$ is regular and hence $(1 / y) d y$ is a representative of a rational differential form on $C$.

Remark 2.6.23 A rational differential form is defined as a collection of equivalent regular differential forms on a collection of open sets. According to Example 2.1.7, open sets on a curve consist of the points on the curve less finitely many points. Thus, any representative of a rational differential form is regular at all but finitely many points of the curve.

The regular differential forms $\Omega[C]$ may be viewed as a subset of the rational differential forms $\Omega(C)$. We just identify a regular differential form $\omega$ with the equivalence class $(\omega, C)$.

Notice that rational differential forms are regular on some open subset of $C$ (the union of the open sets in their class). Since rational functions have this property as well, we may view $\Omega(C)$ as $K(C)$-module.

Lemma 2.6.24 Let $C$ be a projective curve. The rational differential forms on $C$, namely $\Omega(C)$ form a rank 1 module over $K(C)$. In particular, since $K(C)$ is a field, $\Omega(C)$ is a one dimensional vector space over $K(C)$. 
Proof. Consider any open set $U \subset C$, where $\Omega[U]$ is a free rank one module over $K[U]$ as in Theorem 2.6.14. Now, there exists a differential form $d(u(T))$ such that $d(u(T))$ forms a basis of $\Omega[U]$ over $K[U]$. Any rational differential form $\omega \in \Omega(C)$ is regular in some open subset $U^{\prime} \subset U$. Since $U^{\prime} \subset U, d(u(T))_{\mid J^{\prime}}$ is a basis of $\Omega\left[U^{\prime}\right]$ over $K\left[U^{\prime}\right]$. This implies that $\omega$ may be written in the form $\omega=g(T) d(u(T))$ where $g(T)$ is regular on $U^{\prime} \subset U$. Since $U^{\prime}$ is an open subset of $C$ it consists of all but finitely many points of $C$ (Example 2.1.7). Now, $g(T)$ being regular at all but finitely many points of $C$ is equivalent to saying that $g(T)$ is a rational function on $C$ (Corollary 2.1.57). This proves that $d(u(T))$ is a basis for $\Omega(C)$ as a $K(C)$-module.

Corollary 2.6.25 Let $C$ be a projective curve. Let $p$ be a nonsingular point of $C$ and let $s(T)$ be a local parameter at $p$. Then for all rational differential forms $\omega \in \Omega(C)$, we have that $\omega=g(T) d(s(T))$ for some $g(T) \in K(C)$.

Proof. By Proposition 2.6.15, $d(s(T))$ forms a basis of $\Omega[U]$ over $K[U]$ in some neighbourhood $U$ of $p$. Thus replacing $d(u(T))$ by $d(s(T))$ in the above proof gives the desired result.

Let's summarize some of our results. Let $C$ be a nonsingular projective curve. For all $\omega \in \Omega(C)$, by Remark 2.6.23, we know that $\omega$ is regular on some open subset $U \subset C$. Thus, for any nonsingular point $p \in U$, there exists a neighbourhood $U^{\prime} \subset U$ of $p$ such that $\omega=g(T) d(s(T))$ for some $g(T) \in K\left[U^{\prime}\right]$ where $s(T)$ is a local parameter at $p$. Moreover, from Theorem 2.4.10, every point $p$ has an affine neighbourhood where the image of $p$ is nonsingular. So, without loss of generality, it suffices to consider neighbourhoods of nonsingular points of affine plane curves. Doing so allows us to use Proposition 2.6.16, where we showed that $d(s(T))(p) \neq 0$, and so we concluded in Remark 2.6.18, that $\omega(p)=0$ if and only if $g(p)=0$. This allows us to describe the zeros of a rational differential form. If we put a valuation on $\omega$, which we will do shortly, then it makes sense to define $v_{p}(\omega)=v_{p}(g)$. In Corollary 2.6.25, we showed that $\omega$ may be written as $g(T) d(s(T))$ for some $g(T) \in K(C)$. 
Again, since $d(s(T))(p) \neq 0$, it makes sense to define our valuation at all points $p$ to be $v_{p}(\omega)=v_{p}(g(T))$ as well. This is exactly what we will do.

Definition 2.6.26 ([16] p.209) Let $C$ be a projective curve and let $p$ be a nonsingular point on $C$. Let $s(T)$ be a local parameter at $p$ and let $\omega \in \Omega(C)$. By Corollary 2.6.25, $\omega$ may be written in the form $\omega=g(T) d(s(T))$ with $g(T) \in K(C)$. Define the valuation at $p$ of a differential form to be $v_{p}(\omega)=v_{p}(g(T))$.

The definition of $v_{p}(\omega)$ is well-defined; the proof is nearly identical to the one in Remark 3.10.11.

Proposition 2.6.27 Let $C$ be a nonsingular projective curve. A nonzero rational differential form $\omega \in \Omega(C)$ has finitely many zeros and poles.

Proof. By Remark 2.6.23, $\omega$ is regular on some open set $U \subset C$, i.e. $\left.\omega\right|_{U} \in \Omega[U]$. By Lemma 2.6.19, the zeros (or poles) of $\left.\omega\right|_{U}$ form a closed set. Since $U$ consists of all but finitely many points of $C$, there are only finitely many more possible zeros (or poles) of $\omega$. Consequently there are only finitely many zeros in total (Similarly for poles).

This result allows us to form a divisor of a differential form.

Definition 2.6.28 Let $C$ be a nonsingular projective curve and let $\omega \in \Omega(C)$. We define the divisor of a differential form to be

$$
\operatorname{div}(\omega)=\sum_{p \in C} v_{p}(\omega) p
$$

Before we provide an example, we need to make use of the following result.

Lemma 2.6.29 Let $C$ be a projective curve. Let $p$ be a nonsingular point of $C$ and let $z(T) \in \mathcal{O}_{p}$. Then $d(z(T)) \in \Omega(C)$ is regular at $p$ and may be written as $u(T) s(T)^{k} d(s(T)) \in \Omega(C)$, for some unit $u(T) \in \mathcal{O}_{p}$, where $s(T)$ is a local parameter at $p$ and $0 \leq k \in \mathbb{Z}$. 
Proof. Since $z(T)$ is regular at $p, d(z(T)) \in \Omega\left[U_{1}\right]$ for some neighbourhood $U_{1}$ of $p$. Let $U_{2}$ be the neighbourhood of $p$ where $d(s(T))$ is a basis of $\Omega\left[U_{2}\right]$ as in Corollary 2.6.15. Let $U=U_{1} \cap U_{2}$. Then $d(s(T))$ is a basis of $\Omega[U]$, an thus $d(z(T))=$ $v(T) d(s(T))$ for some $v(T) \in K[U]$. Since $v(T) \in K[U]$ it is regular at $p$ and thus by Proposition 2.3.11 may be written as $v(T)=u(T) s(T)^{k}$ for some $0 \leq k \in \mathbb{Z}$. Since $v(T)$ is regular on some open subset of $C$ it is rational on $C$ (Corollary 2.1.57). Therefore $d(z(T))=u(T) s(T)^{k} d(s(T)) \in \Omega(C)$.

In the following example we calculate the divisor of a rational differential form.

Example 2.6.30 Let $C$ be an affine plane curve defined by the polynomial $y^{2}-\varphi(x)$. Let $C^{\prime}$ be a nonsingular projective model of the projective closure $\bar{C}$ of $C$. Let $\omega \in \Omega\left(C^{\prime}\right)$ be such that $\omega=\left(\frac{d x}{y}, C\right)$. As the valuations of rational differential forms are defined over neighbourhoods, we begin by considering the neighbourhoods of the points $\left(x_{0}: y_{0}: 1\right) \in \bar{C}$. These are isomorphic to neighbourhoods of the points $\left(x_{0}, y_{0}\right)$ on $C$ by Defintion 2.1.27. We begin by examining these points of $C$.

Let point $p=\left(x_{0}, y_{0}\right)$ be a point on $C$. If $y_{0} \neq 0$ then by Example 2.3.14, $x-x_{0}$ is a local parameter at $p$. As in Example 2.6.17, the chain rule gives us that $d\left(x-x_{0}\right)=d x$. Combining these results, we have that

$$
v_{p}(\omega)=v_{p}\left(\frac{d x}{y}\right)=v_{p}\left(\frac{d\left(x-x_{0}\right)}{y}\right)=v_{p}\left(\frac{1}{y}\right)
$$

Since $\frac{1}{y}(p)=\frac{1}{y_{0}} \neq 0$ we have that $v_{p}(\omega)=0$.

If $y_{0}=0$ then in Example 2.3.14 we showed that $y$ is a local parameter. Since $y^{2}=\varphi(x)$, taking the differential of both sides we get that

$$
2 y d y=\varphi^{\prime}(x) d x
$$

and so

$$
\frac{d x}{y}=\frac{2}{\varphi^{\prime}(x)} d y
$$


At the points $p=\left(x_{0}, 0\right) \in C$, we know that $\varphi\left(x_{0}\right)=0$. Since $C$ is nonsingular, $\varphi^{\prime}\left(x_{0}\right) \neq 0$ by Example 2.1.13. Therefore, we see that

$$
v_{p}(\omega)=v_{p}\left(\frac{d x}{y}\right)=v_{p}\left(\frac{2}{\varphi^{\prime}(x)} d y\right)=v_{p}\left(\frac{2}{\varphi^{\prime}(x)}\right)
$$

Since $\frac{2}{\varphi^{\prime}(x)}(p)=\frac{2}{\varphi^{\prime}\left(x_{0}\right)} \neq 0$, we have that $v_{p}(\omega)=0$. We have shown that at all points $p=\left(x_{0}: y_{0}: 1\right) \in \bar{C}$ we have that $v_{p}(\omega)=0$. We now have only one more point to consider, $p_{\infty} \in C^{\prime}$, the point birational to $(0: 1: 0)$. By Definition 2.6.28, and from the work above, we know that

$$
\operatorname{div}(\omega)=\sum_{p \in C^{\prime}} v_{p}(\omega) p=v_{p_{\infty}}(\omega) p_{\infty}
$$

We just need to find the value of $v_{p_{\infty}}(\omega)$.

To find the value of $v_{p_{\infty}}(\omega)$ we consider a local parameter $s(x, y)$ at $p_{\infty}$. From Example 2.5.10, $p_{\infty}(x)=2$ and $p_{\infty}(y)=n$. By Lemma 2.3.17 we may express $x$ and $y$ as $x=s(x, y)^{-2} u(x, y)$ and $y=s(x, y)^{-n} v(x, y)$ for some units $u(x, y), v(x, y) \in \mathcal{O}_{p_{\infty}}$ Therefore

$$
\begin{aligned}
\omega=\frac{d x}{y} & =\frac{-2 s(x, y)^{-3} u(x, y) d(s(x, y))+s(x, y)^{-2} d(u(x, y))}{s(x, y)^{-n} v(x, y)} \\
& =\frac{-2 u(x, y) d(s(x, y))+s(x, y) d(u(x, y))}{v(x, y)} s(x, y)^{n-3}
\end{aligned}
$$

Since $u(x, y)$ is a unit, by Lemma $2.6 .29, v_{p_{\infty}}(d(u(x, y))) \geq 0$ and so there exist a unit $h(x, y) \in \mathcal{O}_{p_{\infty}}$ such that $d(u(x, y))=s(x, y)^{k} h(x, y) d(s(x, y))$. This implies that

$$
\omega=\frac{-2 u(x, y)+s(x, y)^{k+1} h(x, y)}{v(x, y)} s(x, y)^{n-3} d(s(x, y)) .
$$

Since $-2 u(x, y)$ is a unit and $s(x, y)^{k+1} h(x, y) \in M_{p_{\infty}}$, the element $w(x, y)=$ 
$-2 u(x, y)+s(x, y)^{k+1} h(x, y)$ is a unit in $\mathcal{O}_{p_{\infty}}$, and $w(x, y) v(x, y)^{-1}$ is too. Finally,

$$
\begin{aligned}
v_{p_{\infty}}(\omega) & =v_{p_{\infty}}\left(w(x, y) v(x, y)^{-1} s(x, y)^{n-3} d(s(x, y))\right. \\
& =v_{p_{\infty}}\left(w(x, y) v(x, y)^{-1} s(x, y)^{n-3}\right) \\
& =n-3
\end{aligned}
$$

and so

$$
\operatorname{div}(\omega)=(n-3) p_{\infty}
$$

\subsection{The Genus of a Curve}

Using the above results we may explore an important birational invariant of a projective curve, namely the genus. As mentioned in the introduction, a hyperelliptic curve is equivalent to a torus with multiple "holes"; the number of which is the genus. However, we will soon provide a rigorous definition of the genus of a curve; one in terms of the space of regular differential forms. Using divisors, we will show that the regular differential forms form a vector space over $K$, and we will define its dimension as the genus. We show that the dimension of the divisor space of any rational differential form is equal to the genus. To compute the genus of hyperelliptic curve, we compute the dimension of such a divisor space.

Proposition 2.7.1 ([16] p.161) Let $C$ be a nonsingular projective curve. The divisors of all rational differential forms on $C$ belong to a unique divisor class on $C$.

Proof. It follows directly from Definition 2.6.26 that for all $g(T) \in K(C)$, and for all $0 \neq \omega \in \Omega(C)$ that

$$
\operatorname{div}(g(T) \omega)=\operatorname{div}(g(T))+\operatorname{div}(\omega) .
$$

Since $\Omega(C)$ is a one-dimensional vector space over $K(C)$ (Lemma 2.6.24), it has a generator $\omega^{\prime}$. This implies that $\omega=h(T) \omega^{\prime}$ for some $h(T) \in K(C)$. Therefore 
$\operatorname{div}(\omega)=\operatorname{div}(h(T))+\operatorname{div}\left(\omega^{\prime}\right)$ where $\operatorname{div}(h(T))$ is a principal divisor. This implies, by Definition 2.5.4, that $\omega$ and $\omega^{\prime}$ are equivalent divisors, i.e. in the same divisor class.

Definition 2.7.2 ([16] p.161) Let $C$ be a nonsingular projective curve. The unique divisor class of $\Omega(C)$ is called the canonical class.

Recall that the regular differential forms my be regarded as a subset of the rational ones. As such, the divisor of a regular differential form is also equivalent to $\operatorname{div}(\omega)$ for any nonzero $\omega \in \Omega(C)$. Also recall from Proposition 2.5.12, that a function is regular if and only if its divisor is $\geq 0$. By the same reasoning as the proposition, this is true of the divisors of regular differential forms. We will use this fact in discussing the space of regular differential forms. First, some preliminaries on effective (positive) divisors.

Definition 2.7.3 ([16] p.161) Let $C$ be a projective curve. Let $D$ be an divisor of $C$. The set consisting of the zero function and all functions $g(T) \in K(C)$ such that $\operatorname{div}(g(T))+D \geq 0$ is called the space of $D$ and is denoted by $L(D)$.

These functions are those whose poles are "no worse" than the zeros of $D$. Notice that $\operatorname{deg}(\operatorname{div}(g(T))+D)=\operatorname{deg}(\operatorname{div}(g(T)))+\operatorname{deg}(D)=\operatorname{deg}(D)$. Thus, if $\operatorname{deg}(D)<0$ then $L(D)=\{0\}$.

Proposition 2.7.4 ([16] p.161) Let $C$ be a projective curve. Let $D$ be a divisor of $C$ then $L(D)$ forms a vector space over $K$.

Proof. Since $D$ is a divisor on $C, D=\sum_{p \in C} n_{p} p$ for some $n_{p} \in \mathbb{Z}$, with only finitely many $n_{p}$ non-zero. Let $g(T), h(T) \in K(C)$ be such that $\operatorname{div}(g(T))+D \geq 0$ and $\operatorname{div}(h(T))+D \geq 0$. By the definition of the partial order of divisors, $\operatorname{div}(g(T))+D \geq 0$ if and only if $v_{p}(g(T))>-n_{p}$ for all $p \in C$, and similarly for $h(T)$. We shall use this fact to verify the properties of a vector space. Let $c \in K$ then

$$
v_{p}(g(T)+h(T)) \geq \min \left\{v_{p}(g(T)), v_{p}(h(T))\right\} \geq-n_{p}
$$




$$
v_{p}(c g(T))=v_{p}(c)+v_{p}(g(T))=v_{p}(g(T)) \geq-n_{p} .
$$

Since this is true for all $p \in C$, both $g(T)+h(T) \in L(D)$ and $c g(T) \in L(D)$. With the zero function in $L(D)$ by definition, it follows easily that $L(D)$ is a vector space.

Recalling Definition 2.5.4, we have the following proposition.

Proposition 2.7.5 ([16] p.161) Equivalent divisors have isomorphic vector spaces.

Proof. Suppose $D_{1} \sim D_{2}$. This means that there exists a $g(T) \in K(C)$ such that $D_{1}-D_{2}=\operatorname{div}(g(T))$. For all $h(T) \in L\left(D_{1}\right)$ we know that $\operatorname{div}(h(T))+D_{1} \geq 0$. Substituting our relation on $D_{1}$ and $D_{2}$, we obtain that

$$
\begin{aligned}
\operatorname{div}(h(T))+D_{1} & \geq 0 \\
\operatorname{div}(h(T))+\left(\operatorname{div}(g(T))+D_{2}\right) & \geq 0 \\
\left.\operatorname{div}(g(T) h(T))+D_{2}\right) & \geq 0
\end{aligned}
$$

i.e. $g(T) h(T) \in L\left(D_{2}\right)$. Therefore, multiplying by $g(T)$ defines a linear map $L\left(D_{1}\right) \rightarrow$ $L\left(D_{2}\right)$. Multiplying by $g(T)^{-1}$ defines a linear map $L\left(D_{1}\right) \rightarrow L\left(D_{2}\right)$, which is clearly the inverse of the first. Therefore $L\left(D_{2}\right)$ and $L\left(D_{1}\right)$ are isomorphic.

Proposition 2.7.5 tells us that the vector spaces of the divisors of any rational differential forms (including regular differential forms) are isomorphic.

Corollary 2.7.6 Let $C$ be a nonsingular projective curve. Then the regular differential forms on $C$ form a vector space over $K$ which is isomorphic to $L(\operatorname{div}(\omega))$ for any $\omega \in \Omega[C]$.

Proof. Let $0 \neq \omega$ be a regular differential form. We prove this result by constructing an isomorphism between $\Omega[C]$ and $L(\operatorname{div}(\omega))$. For all $\omega^{\prime} \in \Omega[C]$ we know that 
$\operatorname{div}\left(\omega^{\prime}\right) \geq 0$. Secondly, since $\Omega(C)$ is a rank $1 K(C)$-module, there exists a function $g(T) \in K(C)$ such that $\omega^{\prime}=g(T) \omega$. This implies that

$$
0 \geq \operatorname{div}\left(\omega^{\prime}\right)=\operatorname{div}(g(T) \omega)=\operatorname{div}(g(T))+\operatorname{div}(\omega)
$$

Therefore $g(T) \in L(\operatorname{div}(\omega))$. Conversely, for all $g(T) \in L(\operatorname{div}(\omega))$, we have that

$$
\begin{aligned}
\operatorname{div}(g(T))+\operatorname{div}(\omega) & \geq 0 \\
\operatorname{div}(g(T) \omega) & \geq 0 .
\end{aligned}
$$

Thus $g(T) \omega \in \Omega[C]$. As such, we have shown that the linear map $\tau: L(\operatorname{div}(\omega)) \rightarrow$ $\Omega[C]$, where $\tau(g(T))=g(T) \omega$, is bijective an thus defines an isomorphism. Consequently $\Omega[C] \cong L(\operatorname{div}(\omega))$.

Using the isomorphism in the above proof we can actually obtain something much stronger.

Corollary 2.7.7 Let $C$ be a nonsingular projective curve and let $\omega_{C}$ be the canonical divisor of $C$. The space of regular differential forms is isomorphic to $L\left(\omega_{C}\right)$.

Theorem 2.7.8 ([16] p.173 and [4] p.193) Let $C$ be a nonsingular projective curve and let $D$ be an effective divisor on $C$. Then the dimension of $L(D)$ as a vector space over $K$ is less than or equal to $\operatorname{deg}(D)+1$.

Proof. Let $p \in C$ be a point appearing in $D$ with multiplicity $r>0$, i.e $D=r p+D_{1}$ where $p$ appears in $D_{1}$ with multiplicity 0 . Set $D^{\prime}=(r-1) p+D_{1}$. Notice that $\operatorname{deg}\left(D^{\prime}\right)=\operatorname{deg}(D)-1$. Since $C$ is nonsingular, by Theorem 2.3.9 there exists a local parameter $s(T)$ at $p$. We now define a linear function $\lambda: L(D) \rightarrow K$. For all $g(T) \in L(D)$ set $\lambda(g(T))=\left(s(T)^{r} g(T)\right)(p)$. This map is properly defined for all $g(T) \in L(D)$. Indeed, by Theorem 2.3.9, $g(T)=s(T)^{m} u(T)$ for some unit $u(T) \in \mathcal{O}_{p}$. 
Since $g(T) \in L(D)$ by Definition 2.7.3 $v_{p}(g(T)) \geq-r$, i.e. $m \geq-r$. As such,

$$
\lambda(g(T))=\left(s(T)^{r} g(T)\right)(p)=\left(s(T)^{r+m} u(T)\right)(p)=s(p)^{r+m} u(p)
$$

Since $m \geq-r, s(p)^{r+m}$ is defined, and since $u(T)$ is a unit in $\mathcal{O}_{p}, u(p)$ is defined (Remark 2.3.3). Therefore $\lambda$ is defined at all points $g(T) \in L(D)$.

The kernel of this map is contained in the subspace $L\left(D^{\prime}\right)$ of $L(D)$. Indeed, suppose $g(T) \in L(D)$ is such that $\lambda(g(T))=0$. Since $g(T)=s(T)^{m} u(T)$ for some $m \geq-r$, and $u(p) \neq 0$ since it is a unit of $\mathcal{O}_{p}$, we have that $\left(s(p)^{r+m} u(p)\right)=0$ implies that $m>-r$. Since $g(T) \in L(D)$ and $v_{p}(g(T)) \geq r-1$, we know that $g(T) \in L\left((r-1) p+D_{1}\right)=L\left(D^{\prime}\right)$.

Hence $L(D) / L\left(D^{\prime}\right)$ is a subspace of $K$, i.e the dimension over $K$ of $L(D) / L\left(D^{\prime}\right) \leq$ 1. We may now repeat this process with $L\left(D^{\prime}\right)$. Since $\operatorname{deg}\left(D^{\prime}\right)=\operatorname{deg}(D)-1$, after $\operatorname{deg}(D)$ iterations we are left to consider $L(0)$. However, $L(0)$ is the set of rational functions that are regular everywhere, i.e. the set of regular functions. Since the regular projective functions are constant we have that $L(0) \cong K$. Since this too is a subspace of $L(D)$, by a repeated use of the first isomorphism theorem $(\operatorname{deg}(D)$ times), we have that $\operatorname{dim}(L(D) / L(0))=\operatorname{dim}(L(D) / K) \leq \operatorname{deg}(D)$. Thus $\operatorname{dim}(L(D)) \leq \operatorname{deg}(D)+1$

Corollary 2.7.9 Let $C$ be a nonsingular projective curve. Then $\Omega[C]$ is a finite dimensional vector space over $K$ of dimension less than or equal to $\operatorname{deg}(\operatorname{div}(\omega))+1$.

Proof. Let $\omega \in \Omega[C]$ be a nontrivial regular differential form. From Corollary 2.7.7 we know that $\Omega[C] \cong L(\operatorname{div}(\omega))$ as vector spaces. From Theorem 2.7.8, we know that $L(\operatorname{div}(\omega))$ is finite dimensional over $K$ and has dimension less than or equal to $\operatorname{deg}(\operatorname{div}(\omega))+1$, and thus so does $\Omega[C]$.

Example 2.7.10 Continuing from Example 2.6.30, we know that the rational differ- 
ential form $\omega=\left(\frac{d x}{y}, C\right)$ is regular on $C^{\prime}$. Subsequently, we proved that

$$
\operatorname{div}(\omega)=(n-3) p_{\infty}
$$

We now consider the space of regular differential forms on $C^{\prime}$ by examining $L(\operatorname{div}(\omega))$. For all $g(x, y) \in L(\operatorname{div}(\omega)), g(x, y)$ may only have one pole, the pole at $p_{\infty}$, and $v_{p_{\infty}}(g) \geq-(n-3)$. For $g(x, y)$ to only have poles at $p_{\infty}, g(x, y)$ must be a polynomial function, i.e. $g(x, y)=a(x)+b(x) y$ for some $a(x), b(x) \in K[x]$. Then

$$
v_{p_{\infty}}(g(x, y))=v_{p_{\infty}}(a(x)+b(x) y) .
$$

Using Example 2.5.10, it is easy to show that $v_{p_{\infty}}(a(x))=-2 \operatorname{deg}(a(x))$ is even and $v_{p_{\infty}}(b(x) y)=-n-2 \operatorname{deg}(b(x))$ is odd. Consequently we may apply the strict triangle inequality (Lemma 2.3.19). This gives us that

$$
\begin{aligned}
v_{p_{\infty}}(g(x, y)) & =v_{p_{\infty}}(a(x)+b(x) y) \\
& =\min \left\{v_{p_{\infty}}(a(x)), v_{p_{\infty}}(b(x) y)\right\} \\
& =\min \{-2 \operatorname{deg}(a(x),-n-2 \operatorname{deg}(b(x))\} .
\end{aligned}
$$

However, for $g(x, y) \in L(\operatorname{div}(\omega))$ we require that $v_{p_{\infty}}(g(x, y)) \geq-(n-3)$. Since

$$
v_{p_{\infty}}(b(x) y)=-n-2 \operatorname{deg}(b(x))<-(n-3)
$$

for all $b(x) \neq 0$, it must be the case that $b(x)=0$, i.e. $g(x, y)=a(x)$. Now, for $a(x) \in L(\omega)$ we require that

$$
v_{p_{\infty}}(a(x))=-2 \operatorname{deg}(a(x)) \geq-(n-3) .
$$

Since $n$ is odd we write $n=2 m+1$. Then for $a(x) \in L(\operatorname{div}(\omega))$, substituting into Equation (2.19), it must be the case that $\operatorname{deg}(a(x)) \leq m-1$. As these conditions are 
both necessary and sufficient,

$$
\Omega\left[C^{\prime}\right] \cong L(\operatorname{div}(\omega))=\{a(x) \in K[x]: \operatorname{deg}(a(x))<m\}
$$

Clearly, $1, x, \ldots, x^{m-1}$ form a basis of $L(\operatorname{div}(\omega))$ over $K$, and so using the isomorphism of Corollary 2.7.6, $\omega, x \omega, \ldots, x^{m-1} \omega$, form a basis of $\Omega\left[C^{\prime}\right]$. Therefore $\operatorname{dim}(L(\omega))=$ $\operatorname{dim}_{K}(\Omega[C])=m$.

Recall from Corollary 2.4.6 that two birationally equivalent nonsingular projective curves are isomorphic. We now show that if two nonsingular projective curves are birationally equivalent then their regular differential forms are isomorphic.

Theorem 2.7.11 Let $C_{1}$ and $C_{2}$ be birationally equivalent nonsingular projective curves. Then $\Omega\left[C_{1}\right]$ and $\Omega\left[C_{2}\right]$ are isomorphic as vector spaces.

Proof. By Corollary 2.4.6, $C_{1}$ and $C_{2}$ are isomorphic. As such, there exists a regular map $\Phi: C_{2} \rightarrow C_{1}$ with a regular inverse $\Phi^{-1}: C_{1} \rightarrow C_{2}$ defined at all points of $C_{1}$ and $C_{2}$ respectively. The regular map $\Phi$ induces a linear map $\Phi^{*}: \Omega\left[C_{1}\right] \rightarrow \Omega\left[C_{2}\right]$ where $\Phi^{*}(h(T) d(g(T)))=\Phi(h(T)) d \Phi(g(T))$ for all $h(T) d(g(T)) \in \Omega\left[C_{1}\right]$. Similarly, the induced map $\left(\Phi^{-1}\right)^{*}$ defines its inverse.

The important thing to take from this theorem is that for nonsingular birationally equivalent projective curves the dimension of the vector space of regular differential forms is the same. This leads us to this very important definition.

Definition 2.7.12 Let $C$ be a projective curve and let $C^{\prime}$ be a nonsingular projective curve birationally equivalent to $C$. Then we define the genus of $C$ to be the dimension over $K$ of the vector space $\Omega\left[C^{\prime}\right]$. We usually write $g_{C}$ for the genus of $C$, i.e. $g_{C}=\operatorname{dim}_{K}\left(\Omega\left[C^{\prime}\right]\right)$

We first note that Theorem 2.4.1 provides us with the existence of such a curve $C^{\prime}$ for each $C$. Secondly, we notice that Theorem 2.7.11 tells us that this definition 
is well defined, since birationally equivalent nonsingular projective curves have the same genus.

Theorem 2.7.13 The genus of a projective curve is invariant under birational equivalence.

Proof. Let $C^{\prime}$ be the nonsingular projective model of a projective curve $C$ (Theorem 2.4.1). Suppose $D$ is birationally equivalent to $C$. Then by transitivity $D$ is birationally equivalent to $C^{\prime}$ and thus the result follows.

There are many equivalent definitions of the genus of a curve. For example, one may define the genus of a projective curve $C$ to be $g_{C}=\operatorname{dim}_{K}(\Omega[C])$. However, one has a more difficult time showing that this definition is invariant under birational equivalence. We give a sketch of the proof here.

Sketch of Proof. Let $C^{\prime}$ be the nonsingular projective model of $C$. By showing that the genus of $C^{\prime}$ is equal to that of $C$, Theorem 2.7.13 gives the desired result. Let $\Phi: C^{\prime} \rightarrow C$ be a birational map. Let $\Phi^{*}: K(C) \rightarrow K\left(C^{\prime}\right)$ be the corresponding field isomorphism (Theorem 2.1.75). This induces an isomorphism $\Phi_{\Omega}: \Omega(C) \rightarrow \Omega\left(C^{\prime}\right)$ where $\Phi_{\Omega}(h(T) d(g(T)))=\Phi^{*}(h(T)) d\left(\Phi^{*}(g(T))\right)$. Let $\omega \in \Omega(C)$. One now shows that $\operatorname{deg}(\operatorname{div}(\omega))=\operatorname{deg}\left(\operatorname{div}\left(\Phi_{\Omega}(\omega)\right)\right)$. Let $S_{P}=\left\{p^{\prime} \in C^{\prime}: \Phi\left(p^{\prime}\right)=p\right\}$. Then by Definition 2.4.9,

$$
\sum_{p \in C} v_{p}(\omega)=\sum_{p \in C} \sum_{p^{\prime} \in S_{p}} v_{p}^{\prime}\left(\Phi_{\Omega}(\omega)\right)=\sum_{p^{\prime} \in C^{\prime}} v_{p}^{\prime}\left(\Phi_{\Omega}(\omega)\right) .
$$

It follows that the degree of the divisor of a rational differential form is invariant under birational equivalence. One then uses a Corollary to the Riemann-Roch Theorem (Theorem 3.10.22) which states that $\operatorname{deg}(\operatorname{div}(\omega))=2 g_{C}-2([16]$ p.210). Thus the genus is invariant under birational equivalence as well.

Example 2.7.14 Continuing from Example 2.7.10, $C$ is an affine plane curve defined by the polynomial $y^{2}-\varphi(x)$ where $\operatorname{deg}(\varphi(x))=2 m+1$. The genus of $\bar{C}$ is $g_{\bar{C}}=$ $m$. Moreover, by Definition 2.7.12, any nonsingular projective curve birationally equivalent to $\bar{C}$ also has gemus $g_{\bar{C}}$. 


\section{Chapter 3}

\section{The Algebraic Perspective}

Since birationally equivalent curves have isomorphic fields of rational functions (Theorem 2.1.75), it makes sense to study rational function fields, disregarding the geometry of the associated curves. This is exactly what we will be doing in this chapter. Furthermore, disregarding the geometry will allow us to relax the condition that $K$ be algebraically closed.

Our study of function fields does have geometric implications. Recall that given a projective plane curve $C$, by Theorem 2.4.1 there exists a nonsingular curve $C^{\prime}$ birationally equivalent to $C$. Also recall from Theorem 2.3.9 that a point $p$ is nonsingular if and only if its valuation ring $\mathcal{O}_{p}$ is a discrete valuation ring. As we will see in the next chapter, for an algebraically closed field $K$, there is a one-to-one correspondence between the discrete valuation rings and points of a nonsingular projective curve. Since birationally equivalent curves have isomorphic rational function fields (Theorem 2.1.75), finding all the discrete valuation rings of the function field $K(C)$, is equivalent to finding all the points of the nonsingular projective curve $C^{\prime}$ birationally equivalent to $C$.

Also, recall that to define the valuation at a singular point $p$ of $C$ (Definition 2.4.9), we used the valuations at the points $p^{\prime} \in C^{\prime}$ which get birationally mapped to $p$. However, we did not give an explicit description of the set points $p^{\prime} \in C^{\prime}$, or 
their valuations. In this chapter, because we will find all the discrete valuation rings $K\left(C^{\prime}\right)$, we will be able to make this explicit. In particular, we will be able to show that the point $p=(0: 1: 0)$ on the projective closure $C$ of an affine plane curve defined by the polynomial $y^{2}-\varphi(x)$, has one point $p^{\prime} \in C^{\prime}$ which maps to it. This fact will complete our description of the points of $C^{\prime}$ and their respective valuations.

Throughout this chapter we let $K$ be a field with $\operatorname{char}(K) \neq 2$, which need not be algebraically closed. The results of this chapter are founded upon [19] and [7].

\subsection{Places}

We begin by investigating the valuation rings of function fields. As mentioned in the previous chapter, discrete valuation rings have maximal principal ideals. In this chapter we will call these ideals "places". We also define the residue field of a place. By the end of this section we will be able to enumerate all the places of the field $K(x)$. We begin with the definition of a function field.

Definition 3.1.1 ([19] p.1) A rational function field in one variable is a field $F$ where

$$
F=K(x)=\left\{\frac{a(x)}{b(x)} \mid b(x) \neq 0\right\}
$$

where $a(x)$ and $b(x)$ are polynomials in $x$ for some $x$ transcendental over $K$.

Definition 3.1.2 ([19] p.1) An algebraic function field $F / K$ is an extension field $F \supseteq K$ such that $F$ is a finite extension of $K(x)$ for some element $x \in F$ which is transcendental over $K . K$ is called the field of constants.

Observe that $K(x)$ is itself an algebraic function field over $K$. We will only be considering algebraic function fields with the same constant field $K$. Thus, we will simply write $F$ for $F / K$. Likewise, since we will not be considering any non-algebraic extensions, we will refer to algebraic function fields as simply function fields. 
Example 3.1.3 In later sections, we will typically be considering the rational function field

$$
K(x)(y)=\frac{K(x)[y]}{\left(y^{2}-\varphi(x)\right)}
$$

where $0 \neq \varphi(x) \in K[x]$. This is an extension of $K(x)$ of degree $\leq 2$ and has $K(x)$ as a subficld so it is clearly an algebraic function field. Following convention, we will simply write this as $K(x, y)$ where $y^{2}=\varphi(x)$ is implicit.

As was the done in the previous chapter, we move on to study the subrings and ideals of an algebraic function field. Recall Definition 2.3.15 of a valuation ring of the rational function field of a curve. We will now give a more general definition of these rings over functions fields.

Definition 3.1.4 ([19] p.2) A valuation ring of the function field $F$ is a ring $\mathcal{O}$ such that $K \varsubsetneqq \mathcal{O} \varsubsetneqq F$ and for any $g \in F, g \in \mathcal{O}$ or $g^{-1} \in \mathcal{O}$.

Let us consider two examples of valuation rings for the function field $K(x)$.

Example 3.1.5 Let $p(x)$ be a monic irreducible polynomial in $K(x)$. Define the ring of $p(x)$, denoted $\mathcal{O}_{p(x)}$, by

$$
\mathcal{O}_{p(x)}:=\left\{\frac{h(x)}{g(x)} \mid h(x), g(x) \in K[x], p(x) \nmid g(x)\right\} .
$$

Let $\frac{a(x)}{b(x)}, \frac{c(x)}{d(x)} \in \mathcal{O}_{p(x)}$. Since $p(x) \nmid b(x)$ and $p(x) \nmid d(x), p(x) \nmid b(x) d(x)$. Consequently $\mathcal{O}_{p(x)}$ is closed under both addition and multiplication and hence $\mathcal{O}_{p(x)}$ is indecd a ring. To show that this is a valuation ring, we assume without loss of generality that $h(x)$ and $g(x)$ are relatively prime. Suppose that $\frac{h(x)}{g(x)} \notin \mathcal{O}_{p(x)}$, i.e. $p(x) \mid g(x)$. This implies that $p(x) \nmid h(x)$ and thus $\frac{g(x)}{h(x)}=\left(\frac{h(x)}{g(x)}\right)^{-1} \in \mathcal{O}_{p(x)}$. Therefore $\mathcal{O}_{p(x)}$ is a valuation ring.

Example 3.1.6 The infinite ring of $K(x)$, denoted $\mathcal{O}_{\infty}$ is defined as

$$
\mathcal{O}_{\infty}:=\left\{\frac{h(x)}{g(x)} \mid h(x), g(x) \in K[x], \operatorname{deg}(h(x)) \leq \operatorname{deg}(g(x))\right\}
$$


To show that this is indeed a ring, let $\frac{a(x)}{b(x)}, \frac{c(x)}{d(x)} \in \mathcal{O}_{\infty}$. Since $\operatorname{deg}(a(x)) \leq \operatorname{deg}(b(x))$ and $\operatorname{deg}(c(x)) \leq \operatorname{deg}(d(x))$, examining the sum of $\frac{a(x)}{b(x)}, \frac{c(x)}{d(x)}$, we see that

$$
\begin{aligned}
\operatorname{deg}(a(x) d(x)+c(x) b(x)) & \leq \max \{\operatorname{deg}(a(x))+\operatorname{deg}(d(x)), \operatorname{deg}(c(x))+\operatorname{deg}(b(x))\} \\
& \leq \operatorname{deg}(b(x))+\operatorname{deg}(d(x))
\end{aligned}
$$

Thus $\mathcal{O}_{\infty}$ is closed under addition. Examining the product of these elements we see that

$$
\operatorname{deg}(a(x) b(x)) \leq \operatorname{deg}(c(x) d(x))
$$

Therefore $\mathcal{O}_{\infty}$ is closed under multiplication. Consequently $\mathcal{O}_{\infty}$ is indeed a ring. To show that this is a valuation ring, suppose $\frac{h(x)}{g(x)} \notin \mathcal{O}_{\infty}$ then $\operatorname{deg}(g(x))<\operatorname{deg}(h(x))$ and thus $\frac{g(x)}{h(x)}=\left(\frac{h(x)}{g(x)}\right)^{-1} \in \mathcal{O}_{\infty}$. Therefore $\mathcal{O}_{\infty}$ is a valuation ring.

As in Chapter 2, valuation rings are local rings, i.e. they have unique maximal ideals.

Proposition 3.1.7 ([19] p.2) Let $\mathcal{O}$ be a valuation ring of the function field $F$. Then $\mathcal{O}$ has a unique maximal ideal $P=\mathcal{O} \backslash \mathcal{O}^{*}$, where $\mathcal{O}^{*}$ is the group of units of $\mathcal{O}$. i.e. $\mathcal{O}$ is a local ring.

Proof. Let $a \in P$ and $b \in \mathcal{O}$. Suppose $a b \notin P$. Then there exists an element $c \in \mathcal{O}$ such that $(a b) c=1$. Then $a(b c)=1$ which implies that $a$ is a unit. This contradicts the fact that $a \in P$. Therefore $P$ is closed under multiplication by $\mathcal{O}$.

Since $\mathcal{O}$ is a valuation ring, either $a / b$ or $b / a$ is in $\mathcal{O}$. Without loss of generality assume that $b / a \in \mathcal{O}$. Then since $\mathcal{O}$ is a ring with unity, $1+b / a \in \mathcal{O}$. So $a+b=a(1+b / a) \in P$ since $a \in P$ and $1+a / b \in \mathcal{O}$. Thus $P$ is closed under addition. Therefore $P$ is an ideal. Since a proper ideal cannot contain a unit, and since $1 \in \mathcal{O}$ and $1 \notin P, P$ is proper and clearly maximal.

Definition 3.1.8 ([19] p.4) A place $P$ of a function field $F$ is the maximal ideal of some valuation $\operatorname{ring} \mathcal{O}$ of $F$. We will denote $\mathcal{P}_{F}$ to be the set of all places $P$ of $F$. 
We will now describe the places for each of the valuation rings in our previous examples.

Example 3.1.9 Continuing from Example 3.1.5, let $p(x) \in K[x]$ be an irreducible polynomial. Let $P_{p(x)}$ be the maximal ideal of $\mathcal{O}_{p(x)}$. Utilizing the definition of $P_{p(x)}$ given in Proposition 3.1.7, we know that $P_{p(x)}$ is the set on non-units of $\mathcal{O}_{p(x)}$. i.e.

$$
\begin{aligned}
P_{p(x)} & =\left\{\frac{h(x)}{g(x)} \mid h(x), g(x) \in K[x], \frac{h(x)}{g(x)} \in \mathcal{O}, \frac{g(x)}{h(x)} \notin \mathcal{O}\right\} \\
& =\left\{\frac{h(x)}{g(x)}|h(x), g(x) \in K[x], p(x) \nmid g(x), p(x)| h(x)\right\} .
\end{aligned}
$$

Remark 3.1.10 In the cases where $p(x)=x-a, a \in K$, i.e. when $p(x)$ is linear, we will often write $P_{a}$ instead of $P_{x-a}$.

Example 3.1.11 Continuing from Example 3.1.6, let $P_{\infty}$ be the maximal ideal of $\mathcal{O}_{\infty}$. Utilizing the definition of $P_{\infty}$ given in Proposition 3.1.7, we know that $P_{\infty}$ is the set on non-units of $\mathcal{O}_{\infty}$, i.e.

$$
\begin{aligned}
P_{\infty} & =\left\{\frac{h(x)}{g(x)} \mid h(x), g(x) \in K[x], \frac{h(x)}{g(x)} \in \mathcal{O}, \frac{g(x)}{h(x)} \notin \mathcal{O}\right\} \\
& =\left\{\frac{h(x)}{g(x)} \mid h(x), g(x) \in K[x], \operatorname{deg}(g(x)) \geq \operatorname{deg}(h(x)), \operatorname{deg}(g(x))>\operatorname{deg}(h(x))\right\} \\
& =\left\{\frac{h(x)}{g(x)} \mid h(x), g(x) \in K[x], \operatorname{deg}(g(x))>\operatorname{deg}(h(x))\right\}
\end{aligned}
$$

Remark 3.1.12 Observe that for a given place $P$ we have that

$$
\mathcal{O}=\left\{f \in F \mid f^{-1} \notin P\right\}
$$

In this way we see that $\mathcal{O}$ is completely determined by $P$. As such, we often write $\mathcal{O}_{P}$ for the valuation ring of the place $P$. Furthermore, utilizing this expression of $\mathcal{O}$ (Equation 3.3), we see that $z \in P$ if and only if $z^{-1} \notin \mathcal{O}$.

We will now demonstrate properties of places $P$. The majority of the properties 
of places that we will be considering are the algebraic generalizations of properties of the maximal ideals of nonsingular points as in Definition 2.3.2.

Theorem 3.1.13 ([19] p.3) Let $\mathcal{O}$ be a valuation ring of the function field $F$ and $P$ be its unique maximal ideal. Then

1. $P$ is a principal ideal i.e $P=t \mathcal{O}$ for some $t \in \mathcal{O}$

2. Any $0 \neq z \in F$ has a unique representation of the form $z=t^{k} u$ for some $k \in \mathbb{Z}, u \in \mathcal{O}^{\times}$.

This proof requires the following lemma.

Lemma 3.1.14 ([19] p.3) Let $\mathcal{O}$ be a valuation ring of the function field $F$. Let $P$ be its maximal ideal and let $0 \neq z \in P$. Let $x_{1}, \ldots, x_{m} \in P$ be such that $x_{1}=z$ and $x_{i} \in x_{i+1} P$ for $i=1, \ldots, m-1$. Then, we have that $m \leq[F: K(z)]$. i.e. we may have at most $[F: K(z)]$ elements with this property.

Proof. Since $F$ may be viewed as a vector space over $K(z)$ of dimension $[F: K(z)]$, it is enough for us to show that $x_{1}, \ldots, x_{m}$ are independent over $K(z)$. After all, their independence guarantees that $m<[F: K(z)]$. By contradiction, we suppose that there exists a non-trivial linear combination $\sum_{i=1}^{m} g_{i}(z) x_{i}=0$ with $g_{i}(z) \in K(z)$. After multiplying by a common denominator and factoring we may assume that the $g_{i}(z)$ are polynomials and that $z$ does not divide all of them. Let $a_{i}=g_{i}(0)$ be the constant term of $g_{i}(z)$. Now there exists a $j \in\{1, \ldots, m\}$ with the condition that $a_{i}=0$ for all $i>j$. Consequently, we obtain

$$
\begin{aligned}
-g_{j}(z) x_{j} & =\sum_{i \neq j} g_{i}(z) x_{i} \\
-g_{j}(z) & =\sum_{i<j} g_{i}(z) x_{i} x_{j}^{-1}+\sum_{i>j} g_{i}(z) x_{i} x_{j}^{-1} .
\end{aligned}
$$

We examine the properties of each of the sums on the right. In the first sum, since $z=x_{1} \in P$ and $x_{i} \in x_{j} P$ for all $i<j$, we have that $x_{j}^{-1} x_{i} \in P$ for $i<j$. In the 
second sum, since $g_{i}(0)=0$ for all $i>j$, we have that $g_{i}(z)=z h_{i} \in P$ for $i>j$ and so $g_{i}(z) x_{i} x_{j}^{-1} \in P$ for all $i>j$. Thus

$$
\sum_{i<j} g_{i} x_{i} x_{j}^{-1}+\sum_{i>j} g_{i} x_{i} x_{j}^{-1}=-g_{j} \in P .
$$

However, $g_{j}=a_{j}+z h_{j}$ for some $h_{j} \in K[z]$. Since $z \in P$, we have that $z h_{j} \in P$, i.e. $a_{j}=g_{j}-z h_{j} \in P$. However $0 \neq a_{j} \in K$ and is thus a unit. This means that $P$ contains a unit, but this contradicts the definition of a place (Proposition 3.1.7), as a proper ideal. Thus, a contradiction is reached and the lemma is proven.

We now prove the theorem.

Proof. (of Theorem 3.1.13)

1. Assume that $P$ is not principal and choose and element $0 \neq x_{i} \in P$. As $P \neq x_{1} \mathcal{O}$ there exist an element $x_{2} \in P \backslash x_{1} \mathcal{O}$. Then $x_{2} \notin x_{1} \mathcal{O}$, or equivalently $x_{2} x_{1}^{-1} \notin \mathcal{O}$. From Remark 3.1.12, this implies that $\left(x_{2} x_{1}^{-1}\right)^{-1} \in P$, i.e. $x_{2}^{-1} x_{1} \in P$; or equivalently $x_{1} \in x_{2} P$. By repeating this process, we obtain an infinite sequence $x_{1}, x_{2}, \ldots$ in $P$ such that $x_{i} \in x_{i+1} P$ for all $i>1$. This contradicts lemma 3.1.14, and so $P$ is principal.

2. We first prove the uniqueness. Suppose that $z=u t^{n}=v t^{m}$ where $u$ and $v$ are units. Without loss of generality suppose $n \geq m$. Then $u t^{n-m}=v$ is a unit. However, $t \in P$ is not a unit and so it must be the case that $n=m$ and consequently $u=v$. Now we need to prove the existence of this expression. If $z \in \mathcal{O}^{*}$ then $z=z t^{0}$. If $z \in P$ then there is a maximal $m \geq 1$ with $z \in t^{m} P$ since the sequence

$$
x_{1}=z, x_{2}=t^{m-1}, x_{3}=t^{m-2}, \ldots, x_{m}=t
$$

is bounded by Lemma 3.1.14. Write $z=t^{m} u$ with $u \in \mathcal{O}$. Now $u$ must be a unit since otherwise if $u \in P=t \mathcal{O}$ then $u=t v$ with $v \in \mathcal{O}$. Consequently 
$z=t^{m+1} \in t^{m+1} \mathcal{O}$ which would contradict the maximality of $m$. Lastly, if $z \notin \mathcal{O}$ then by Remark 3.1.12 $z^{-1} \in P$. By the above result, $z^{-1}=t^{m} u$ and thus $z=t^{-m} u^{-1}$ as desired.

As in Chapter 2, we give a name to an element that generates the maximal ideal of a local ring.

Definition 3.1.15 ([19] p.4) Let $P$ be a place of the function field $F$. Then an element $t$ such that $P=t \mathcal{O}$ is called a local parameter or uniformizing parameter.

Example 3.1.16 In this example, we will examine the local parameters at each of the places $P_{p(x)}$ and $P_{\infty}$ of $K(x)$.

- Continuing from Example 3.1.9, for all $\frac{a(x)}{b(x)} \in P_{p(x)}$ we have that $p(x) \mid a(x)$ and $p(x) \nmid b(x)$. Since $K[x]$ is a Unique Factorization Domain, there exists a unique $0<m \in \mathbb{Z}$ such that $a(x)=p(x)^{m} q(x)$ for some $q(x) \in K[x]$ such that $p(x) \nmid q(x)$. Therefore $p(x)$ is a local parameter and we may express $\frac{a(x)}{b(x)}$ as $p(x)^{m} \frac{q(x)}{b(x)}$ where $\frac{q(x)}{b(x)} \in \mathcal{O}_{p(x)}^{*}$.

- Continuing from Example 3.1.11, for all $\frac{a(x)}{b(x)} \in P_{\infty}$ we have that $\operatorname{deg}(a(x))<$ $\operatorname{deg}(b(x))$. Let $m=\operatorname{deg}(b(x))-\operatorname{deg}(a(x))$. Then we may express $\frac{a(x)}{b(x)}$ as $x^{-m} \frac{x^{m} a(x)}{b(x)}$ where $\operatorname{deg}\left(x^{m} a(x)\right)=\operatorname{deg}(b(x))$. Therefore $x^{-1}$ is a local parameter at $P_{\infty}$ and we may express every $\frac{a(x)}{b(x)} \in \mathcal{O}_{\infty}$ as $x^{-m} \frac{x^{m} a(x)}{b(x)}$ where $\frac{x^{m} a(x)}{b(x)} \in \mathcal{O}_{\infty}^{*}$.

There is one more algebraic structure associated to a place of a rational function field that is of particular interest to us.

Definition 3.1.17 ([19] p.6) Let $P$ be a place with valuation ring $\mathcal{O}_{P}$ in the function field $F$. Set $F_{P}=\mathcal{O}_{P} / P$. Since $P$ is a maximal ideal, $F_{P}$ is a field and is called the residue class field of $P$. We also define the degree of $P$ by $\operatorname{deg}(P)=\left[F_{P}: K\right]$.

Lemma 3.1.18 ([19] p.6) Let $P$ be a place with valuation ring $\mathcal{O}_{P}$ in the function field $F$. Then $\operatorname{deg}(P)=\left[F_{P}: K\right]<\infty$. 
Proof. Recall that we are only considering finite extensions $F$ of $K(x)$. Since $P \neq 0$, there exists a nonzero $z \in P$. Now since $z \in K(x),[K(x): K(z)]<\infty$ and so

$$
[F: K(z)]=[F: K(x)][K(x): K(z)]<\infty
$$

Consequently, we need only to prove that $\left[F_{P}: K\right] \leq[F: K(z)]$. Let $z_{1}+P, \ldots, z_{n}+P$ be a linearly independent set in $F_{P}$ over $K$. Suppose that there exists a non-trivial linear combination

$$
\sum_{i=1}^{n} k_{i}(z) z_{i}=0
$$

where $k_{i}(z) \in K(z)$ for all $i=1, \ldots, n$. By clearing denominators we may assume that all $k_{i}(z)$ 's are polynomials in $K[z]$. We may also assume that not all $k_{i}(z)$ 's are divisible by $z$. Therefore, without loss of generality, let $k_{i}(z)=a_{i}+z g_{i}(z)$ for some $a_{i} \in K, g_{i}(z) \in K[z]$, where not all $a_{i}=0$. Applying the quotient map to Equation (3.5) we obtain

$$
0=\sum_{i=1}^{n} k_{i} z_{i}+P=\sum_{i=1}^{n}\left(a_{i}+z g_{i}(z)\right) z_{i}+P=\sum_{i=1}^{n} a_{i} z_{i}+P
$$

This contradicts the independence of $z_{1}+P, \ldots, z_{n}+P$ over $K$. Therefore

$$
\left[F_{P}: K\right] \leq[F: K(z)]<\infty
$$

as required.

We now turn our attention to the residue class fields of the valuation rings discussed in our earlier examples.

Example 3.1.19 Continuing from Example 3.1.16, we examine the residue class field of first $P_{p(x)}$ and then $P_{\infty}$.

1. Let $P=P_{p(x)}$. We will examine the relation between $F_{P}$ and the polynomial ring $K[x]$. We define the ring homomorphism $\Phi: K[x] \rightarrow F_{P}$ as $\Phi(f(x))=f(x)+P$. 
Then certainly

$$
\operatorname{ker}(\Phi)=\{f(x) \mid f(x) \in P\}=\{f(x)|f(x) \in K[x], p(x)| f(x)\}=(p(x)) .
$$

Next, we show that $\Phi$ is surjective and use the First Isomorphism Theorem of rings. Let $z \in \mathcal{O}_{P}$. Then we may write $z=h(x) / g(x)$ where $h(x), g(x) \in K[x]$, and $p(x) \nmid g(x)$. Since $K[x]$ is a Euclidean Domain there exists $a(x), b(x) \in K[x]$ such that $a(x) p(x)+b(x) v(x)=1$. Therefore

$$
z+P=z \cdot 1+P=\left(\frac{a(x) u(x)}{v(x)} p(x)+a(x) u(x)\right)+P=a(x) u(x)+P .
$$

So for all $z+P \in F_{P}$ there exists $a(x) u(x) \in K[x]$, as above, such that $\Phi(a(x) u(x))=z+P$. By the first isomorphism theorem of rings $K[x] /(p(x)) \cong$ $F_{P}$ and $\operatorname{sodeg}(P)=\left[F_{P}: K\right]=[K[x] /(p(x)): K]=\operatorname{deg}(p(x))$.

2. Define ring homomorphism $\Phi: \mathcal{O}_{P} \rightarrow K$ where

$$
\Phi(g(x) / h(x))=\left\{\begin{array}{cc}
g_{n} / h_{n} & \operatorname{deg}(g(x))=\operatorname{deg}(h(x)) \\
0 & \operatorname{deg}(g(x)<\operatorname{deg}(h(x))
\end{array} .\right.
$$

where $g_{n}$ and $h_{n}$ are the leading coefficients of $g(x)$ and $h(x)$ respectively. Clearly $\Phi$ is surjective. Moreover $\operatorname{ker}(\Phi)=P_{\infty}$, and so by the first isomorphism theorem for rings $F_{P} \cong K$, i.e. $\left[F_{P}: K\right]=1$.

All of our examples have been about only two types of places of $K(x)$. This is due to the following theorem.

Theorem 3.1.20 ([19] p.10) There are no places of the rational function field $K(x)$ other than the places $P_{p(x)}$ and $P_{\infty}$ defined in Example 3.1.9 and Example 3.1.11.

To prove this we need to make use of the following Lemma.

Lemma 3.1.21 ([19] p.5) Any valuation ring $\mathcal{O}$ of a function field $F$ is a maximal proper subring of $F$. 
Proof. Let $\mathcal{O}_{P}$ be a valuation ring of a function field $F$ with maximal ideal $P$. Let $t$ be a local parameter at $P$ (Definition 3.1.15). Let $z \in F \backslash \mathcal{O}$, then we need only to prove that $F=\mathcal{O}[z]$. Since $z \notin \mathcal{O}, z^{-1} \in P$ by Remark 3.1.12. This means that $z^{-1}=t^{m} u$ for some $0<m \in \mathbb{Z}$ and some unit $u \in \mathcal{O}$. Let $g \in F$ and so we need to show $g \in \mathcal{O}[z]$. By Theorem 3.1.13, $g=t^{n} v$ for some $n \in \mathbb{Z}$ and some unit $v \in \mathcal{O}_{p}$. Then

$$
g z^{-k}=t^{n} v \cdot t^{m k} u^{k}=t^{n+m k} u^{k} v .
$$

So for sufficiently large $k, n+m k>0$ and thus $g z^{-k} \in \mathcal{O}$. Define $w:=g z^{-k} \in \mathcal{O}$. Then $g=w z^{k} \in \mathcal{O}[z]$ and therefore $F=\mathcal{O}[z]$ for all $z \in F \backslash \mathcal{O}$, and so $\mathcal{O}$ is a maximal proper subring of $F$.

We may now prove Theorem 3.1.20.

Proof. (of Theorem 3.1.20)

Let $\mathcal{O}_{P}$ be a valuation ring of $F$ where $P$ is its maximal ideal. Since $K(x) \subseteq F$, there are two cases to consider.

1. Assume that $x \in \mathcal{O}_{P}$. Then $K[x] \subseteq \mathcal{O}_{P}$. Set $I:=K[x] \cap P$ then $I$ is an ideal of $K[x]$. There is a natural embedding $\Phi: K[x] / I \hookrightarrow \mathcal{O}_{P} / P$ where $\Phi(g(x)+I)=g(x)+P$. If $I=0$ then $K[x] \cong \mathcal{O}_{P} / P$ which means that $K[x]$ is a field; a contradiction. Therefore $I \neq 0$. Since $K[x]$ is a principal ideal domain, there exists a $p(x) \in K[x]$ such that $\langle p(x)\rangle=I$. Now for any $g(x) \in K[x]$ with $p(x) \nmid g(x), g(x) \notin I$ and so $g(x) \notin P \subset \mathcal{O}_{P}$. Since $\mathcal{O}_{P}$ is a valuation ring, $1 / g(x) \in \mathcal{O}_{P}$. From above we have that $K[x] \subseteq \mathcal{O}_{P}$, so we may conclude that

$$
\mathcal{O}_{p(x)}=\left\{\frac{h(x)}{g(x)} \mid h(x), g(x) \in K[x], p(x) \nmid g(x)\right\} \subseteq \mathcal{O}_{P} .
$$

From Lemma 3.1.21 we know that valuation rings are maximal proper subrings of $F$ and therefore $\mathcal{O}_{p(x)}=\mathcal{O}_{P}$.

2. Assume that $x \notin \mathcal{O}_{P}$. Then $x^{-1} \in \mathcal{O}_{P}$ and $K\left[x^{-1}\right] \subseteq \mathcal{O}_{P}$. Then as above, 
we define $I:=K\left[x^{-1}\right] \cap P$ and the embedding $\Phi: K\left[x^{-1}\right] / I \hookrightarrow \mathcal{O}_{P} / P$ where $\Phi\left(g\left(x^{-1}\right)+I\right)=g\left(x^{-1}\right)+P$. Since $x \notin \mathcal{O}_{P}, x^{-1}$ is not a unit in $\mathcal{O}_{P}$ and thus $x^{-1} \in P$. Since $x^{-1}$ generates $K\left[x^{-1}\right]$, it generates $I=P \cap K\left[x^{-1}\right]$ and so $I=\left\langle x^{-1}\right\rangle$. Now for any $g\left(x^{-1}\right) \in K\left[x^{-1}\right]$ with $x^{-1} \nmid g\left(x^{-1}\right), g\left(x^{-1}\right) \notin I$ and so $g\left(x^{-1}\right) \notin P \subset \mathcal{O}_{P}$. Since $\mathcal{O}_{P}$ is a valuation ring, $1 / g\left(x^{-1}\right) \in \mathcal{O}_{P}$. From above we have that $K\left[x^{-1}\right] \subseteq \mathcal{O}_{P}$, so we may conclude that

$$
\begin{aligned}
\mathcal{O}_{P} & \supseteq\left\{\frac{h\left(x^{-1}\right)}{g\left(x^{-1}\right)} \mid h\left(x^{-1}\right), g\left(x^{-1}\right) \in K\left[x^{-1}\right], x^{-1} \nmid g\left(x^{-1}\right)\right\} \\
& =\left\{\frac{h_{0}+h_{1} x^{-1}+\ldots+h_{n} x^{-n}}{g_{0}+g_{1} x^{-1}+\ldots+g_{m} x^{-m}} \mid g_{0} \neq 0\right\} \\
& =\left\{\frac{h_{0} x^{n+m}+\ldots+h_{n} x^{m}}{g_{0} x^{n+m}+\ldots+g_{m} x^{n}} \mid g_{0} \neq 0\right\} \\
& =\left\{\frac{a(x)}{b(x)} \mid a(x), b(x) \in K[x], \operatorname{deg}(a(x)) \leq \operatorname{deg}(b(x))\right\} \\
& =\mathcal{O}_{\infty} .
\end{aligned}
$$

From Lemma 3.1.21 we know that valuation rings are maximal proper subrings of $F$ and therefore $\mathcal{O}_{P}=\mathcal{O}_{\infty}$.

These two cases are clearly exhaustive and thus the theorem is proven.

Over an algebraically closed field the above result of Theorem 3.1.20 is not surprising. The irreducible polynomials over $K(x)$ are all linear, i.e. of the form $x-a$ for some $a \in K$. Thus the places $P_{p(x)}$ are in one-to-one correspondence with the points $(a: 1)$ of $\mathbb{P}^{1}$. The place $P_{\infty}$ corresponds to the projective point $(1: 0)$ giving a one-to-one correspondence between the places of $K(x)$ and the points of $\mathbb{P}^{1}$. This should be of no great surprise as $K(x)$ is isomorphic to the rational functions on the curve $\mathbb{P}^{1}$, i.e. $K\left(\mathbb{P}^{1}\right) \cong K(x)$.

It should not be surprising that we may use local rings on curves to describe some of the places of the function field $K(x, y)$ where $y$ satisfies $c(x, y)=0$ for some $c(x, y) \in K[x, y]$. 
Example 3.1.22 Just as we did for local rings at a point on a curve, we may use evaluation to describe some places of an algebraic extension of $K(x)$. Let $K(x, y)$ be an algebraic extension of $K(x)$. Since $y$ is algebraic there exists some $c(x, y) \in$ $K[x, y]$ such that $c(x, y)=0$. We will assume that there exist $p_{1}, p_{2} \in K$ such that $c\left(p_{1}, p_{2}\right)=0$. This is very much like assuming that there is a point $p=\left(p_{1}, p_{2}\right)$ on the curve defined by $c(x, y)$. We also assume that $p$ is nonsingular, i.e. not both of $\frac{\partial c}{\partial x}(p)$ and $\frac{\partial c}{\partial y}(p)$ are zero. Then, just as we did in Section 2.3 we define the place $P$ and the ring $\mathcal{O}_{P}$ as

$$
\begin{aligned}
\mathcal{O}_{P} & =\left\{\frac{a(x, y)}{b(x, y)} \in K(x, y) \mid b\left(p_{1}, p_{2}\right) \neq 0\right\} \\
P & =\left\{\frac{a(x, y)}{b(x, y)} \in K(x, y) \mid a\left(p_{1}, p_{2}\right)=0, b\left(p_{1}, p_{2}\right) \neq 0\right\}
\end{aligned}
$$

Just as in Proposition 2.3.16, $\mathcal{O}_{P}$ is a valuation ring. Since $P$ is the kernel of the evaluation homomorphism, $\mathcal{O}_{P} / P \cong K$ and $P$ is a maximal ideal. By Definition 3.1.8 $P$ is a place of $K(x, y)$.

As we will see later on, when $K$ is not algebraically closed, $K(x, y)$ may have places that are not identified with points on a curve. These places are related to the place $P_{p(x)}$ of $K(x)$ where $p(x)$ is an irreducible polynomial of degree greater than one.

\subsection{Valuations, Absolute Values and Completions}

Just as we did for valuation rings of the rational functions of a curve in Definition 2.3.20, we will define a valuation function. With valuations we will also define another function on a curve, namely an absolute value. Just as in analysis, absolute values measure "distance" between elements. This "distance" measurement then allows us to look sequences of elements and their convergence. The results in this section are for valuation rings on any function field. However, as we do not yet have the means 
to describe the places of non-trivial extensions of $K(x)$ explicitly, the examples in this section will only be over $K(x)$. Subsequently, this section concludes with a description of the completion of each place of $K(x)$.

\subsubsection{Discrete Valuations}

Generalizing what we did for local rings at points in Definition 2.3.20, we define valuations on general function fields.

Definition 3.2.1 ([19] p.4) A discrete valuation of a function field $F$ is a function $v: F \rightarrow \mathbb{Z} \cup \infty$ such that

1. $v(x)=\infty$ if and only if $x=0$.

2. $v(x y)=v(x)+v(y)$ for all $x, y \in F$.

3. $v(x+y) \geq \min \{v(x), v(y)\}$ for all $x, y \in F$.

4. $v(a)=0$ for any $0 \neq a \in K$.

Lemma 3.2.2 (Strict Triangle Inequality) ([19] p.5) Let $v$ be a discrete valuation of $F$ and let $a, b \in F$ be such that $v(a) \neq v(b)$. Then $v(a+b)=\min \{v(a), v(b)\}$.

Proof. The proof is nearly identical to the proof of Lemma 2.3.19.

Theorem 3.2.3 ([19] p.5) Let $F$ be a function field. For any place $P \in P_{F}$ there exists a discrete valuation $v_{P}$ such that

$$
\begin{aligned}
\mathcal{O}_{P} & =\left\{z \in F \mid v_{P}(z) \geq 0\right\}, \\
\mathcal{O}_{P}^{*} & =\left\{z \in F \mid v_{P}(z)=0\right\}, \\
P & =\left\{z \in F \mid v_{P}(z)>0\right\}
\end{aligned}
$$


Proof. Let $t$ be a local parameter at $P$ as in Definition 3.1.15. Then by Theorem 3.1.13, for all $z \in F, z=t^{m} u$ for some unit $u \in \mathcal{O}_{P}$. Define

$$
v_{P}(z)= \begin{cases}m, & \text { if } z \neq 0 \\ \infty & \text { if } z=0\end{cases}
$$

Clearly we have that $z \in \mathcal{O}_{P}$ if and only if $v_{P}(z) \geq 0$. We need only prove that this is indeed a discrete valuation. The first, second and last conditions of Definition 3.2.1 are trivial. This leaves us only with the third criterion to verify.

Let $z_{1}, z_{2} \in F$ where $v_{P}\left(z_{1}\right)=n_{1}$ and $v_{P}\left(z_{2}\right)=n_{2}$. We assume without loss of generality that $n_{1} \leq n_{2}$. Let $z_{1}=t^{n_{1}} u_{1}$ and $z_{2}=t^{n_{2}} u_{2}$ for some units $u_{1}, u_{2} \in \mathcal{O}_{P}$. Then

$$
\begin{aligned}
v_{P}\left(z_{1}+z_{2}\right) & =v_{P}\left(t^{n_{1}} u_{1}+t^{n_{2}} u_{2}\right) \\
& =v_{P}\left(t^{n_{1}}\left(u_{1}+t^{n_{2}-n_{1}} u_{2}\right)\right) \\
& =n_{1}+v_{P}\left(u_{1}+t^{n_{2}-n_{1}} u_{2}\right) .
\end{aligned}
$$

As $u_{1}+t^{n_{2}-n_{1}} u_{2} \in \mathcal{O}_{P}$, we know that $v_{P}\left(u_{1}+t^{n_{2}-n_{1}} u_{2}\right) \geq 0$. Consequently,

$$
\begin{aligned}
v_{P}\left(z_{1}+z_{2}\right) & =n_{1}+v_{P}\left(u_{1}+t^{n_{2}-n_{1}} u_{2}\right) \\
& \geq n_{1} \\
& \geq \min \left\{n_{1}, n_{2}\right\} \\
& \geq \min \left\{v_{P}\left(z_{1}\right), v_{P}\left(z_{2}\right)\right\} .
\end{aligned}
$$

Thus $v_{P}$ is a discrete valuation.

The valuation at a place is well-defined, i.e. it is independent of the choice of local parameter. Indeed, the proof of this is nearly the same as the proof that the valuation at a point is well-defined (Definition 2.3.20). 
Corollary 3.2.4 Let $F$ be a function field and $P \in \mathcal{P}_{F}$. Then $t$ is a local parameter at $P$ (Definition 3.1.15) if and only if $v_{P}(t)=1$.

Proof. If $t$ is a local parameter at $P$ then by Definition 3.2.1, $v_{P}(t)=1$. Conversely, assume that $v_{P}(z)=1$ for some $z \in F$. Then by the proof of Theorem 3.2.3, there exist a unit $u$ such that $z=t u$. Now, for all $g \in P$, by Theorem 3.1.13, $g=t h$ for some $h \in \mathcal{O}_{P}$. Substituting in for $t$, we obtain that $g=z u^{-1} h$. Since $u$ is a unit $u^{-1} h \in \mathcal{O}_{P}$. Therefore $z$ is a generator of $P$, and by Definition 3.1.15 a local parameter at $P$.

Definition 3.2.5 Let $z \in F$ where $F$ is function field. We know from Theorem 3.2.3 that for any place $P \in \mathcal{P}_{F}$ there exists a discrete valuation $v_{P}$. We say that $z$ has a zero at $P$ if $v_{P}(z)>0$, and alternatively we say that $z$ has a pole at $P$ if $v_{P}(z)<0$.

Corollary 3.2.6 ([19] p.9) Let $F$ be the function field $K(x)$. Let $p(x) \in K[x]$ be an irreducible polynomial.

1. Let $P=P_{p(x)}$ as in Example 3.1.5. For all $z(x) \in K(x) \backslash\{0\}, z(x)$ may be written in the form $z=p(x)^{n}(a(x) / b(x))$ with $n \in \mathbb{Z}, a(x), b(x) \in K[x]$ where $p(x) \nmid a(x)$ and $p(x) \nmid b(x)$. Define $v_{P}: \mathcal{P}_{K(x)} \rightarrow \mathbb{Z} \cup \infty$ by

$$
v_{P}(z)= \begin{cases}n, & \text { if } z \neq 0 \\ \infty, & \text { if } z=0\end{cases}
$$

Then $v_{P}$ is a discrete valuation.

2. Let $P=P_{\infty}$ as in Example 3.1.6. For all $z(x) \in K(x) \backslash\{0\}, z(x)$ may be written in the form $z=a(x) / b(x), a(x), b(x) \in K[x]$. Define $v_{P}: \mathcal{P}_{K(x)} \rightarrow \mathbb{Z} \cup \infty$ as

$$
v_{P}(a(x) / b(x))= \begin{cases}\operatorname{deg}(b(x))-\operatorname{deg}(a(x)), & \text { if } z \neq 0 \\ \infty, & \text { if } z=0\end{cases}
$$

Then $v_{P}$ is a discrete valuation. 
Proof. In both cases the result follows almost directly from the proof of Theorem 3.2 .3 .

1. From Example 3.1.16, $p(x)$ is a local parameter at $P_{p(x)}$, so the result follows directly.

2. Again, from Example 3.1.16, we saw that $x^{-1}$ is local parameter at $P_{\infty}$, so again the result follows directly.

Corollary 3.2.7 Let $F$ be a function field. There is a one-to-one correspondence between the discrete valuations of $F$ and the places of $F$.

Proof. From Theorem 3.2.3, to each place $P$ in $F$ we may define a discrete valuation. Alternatively, given a discrete valuation $v$ we may define a place $P$ as $P=\{z \in F \mid v(z)>0\}$. This is an ideal of the ring $\mathcal{O}=\{z \in F \mid v(z) \geq 0\}$. Indeed, by

the properties of a discrete valuation $\mathcal{O}$ is a ring. In addition, since $v_{P}\left(z^{-1}\right)=-v_{P}(z)$, we have that $\mathcal{O}$ is a valuation ring. Now, by design, for all $z \in \mathcal{O}, v_{P}(z) \geq 0$, and $z$ is a unit if and only if $z^{-1} \in \mathcal{O}_{P}$. As $z^{-1} \in \mathcal{O}_{P}$ implies that $v_{P}\left(z^{-1}\right)=-v_{P}(z) \leq 0$, we have that $z$ is a unit if and only if $v_{P}(z)=0$. Therefore $P \subset \mathcal{O}_{P}$ is the set of non-units and hence the unique maximal ideal, which by definition is a place. This gives the one-to-one correspondence.

\subsubsection{Absolute Values}

Using valuations we may now define absolute values. Absolute values provide us with a means to measure "distance" between elements of a field.

Definition 3.2.8 ([7] p.558) Let $F$ be a function field. A function $\Phi: F \rightarrow \mathbb{R} \geq 0$ is called an absolute value if it satisfies the following properties.

1. $\Phi(z)=0 \Leftrightarrow z=0$

2. $\Phi(z w)=\Phi(z) \Phi(w) \quad \forall z, w \in F$ 
3. $\Phi(z+w) \leq \Phi(z)+\Phi(w) \quad \forall z, w \in F$

We say that two absolute values $\Phi, \Phi^{\prime}$ are equivalent if there exists some $0<c \in \mathbb{R}$ such that for all $z \in F$ we have that $\Phi(z)=\left(\Phi^{\prime}(z)\right)^{c}$.

To each place $P$ we may define an absolute value.

Definition 3.2.9 Let $F$ be a function field. Define $\Phi_{P}: F \rightarrow \mathbb{R}$ by $\Phi_{P}(z)=$ $(1 / 2)^{v_{P^{\prime}}(z)}$. We call $\Phi_{P}$ the absolute value of $P$ and denote $\Phi_{P}(z)$ as $|z|_{P}$.

Let's examine some properties of absolute values.

Example 3.2.10 Let $F$ be a function field. Let $P \in \mathcal{P}_{F}$ and let $t$ be a local parameter of $P$. For all $z \in F, z=t^{n} u$ for some unit $u$ in $\mathcal{O}_{P}$. Then $|z|_{P}=(1 / 2)^{n}$.

1. Now suppose that $\left|z_{2}\right|_{P}<|z|_{P}$ for some $z_{2} \in F$. This means that $(1 / 2)^{v_{P}\left(z_{2}\right)}<$ $(1 / 2)^{v_{P}(z)}$. i.e $v_{P}(z)<v_{P}\left(z_{2}\right)$. Thus smaller absolute values correspond to larger valuations.

2. Suppose that $z$ is a unit. Then

$$
|z|_{P}=(1 / 2)^{v_{P}(z)}=(1 / 2)^{0}=1
$$

3. Let $c \in K$ then

$$
|c z|_{P}=|c||z|_{P}=(1 / 2)^{v_{P}(c)}|z|_{P}=(1 / 2)^{0}|z|_{P}=|z|_{P}
$$

4. Let $z, w \in F$. Then

$$
\begin{aligned}
|z+w|_{P} & =(1 / 2)^{v_{P}(z+w)} \\
& \leq(1 / 2)^{\min \left\{v_{P}(z), v_{P}(w)\right\}} \quad \text { by Definition 3.2.1 } \\
& =\max \left\{|z|_{P},|w|_{P}\right\}
\end{aligned}
$$

which is even stronger than the triangle inequality and is hence referred to as the strong triangle inequality. 
Suppose that there exists another absolute value $\|$ equivalent to $\|_{P}$. Then there exists a real number $b \in \mathbb{R}$ such that for all $z \in F$

$$
|z|=\left(|z|_{P}\right)^{b}=\left((1 / 2)^{v_{P}(z)}\right)^{b}=\left((1 / 2)^{b}\right)^{v_{P}(z)}
$$

So absolute values equivalent to $\|_{P}$ just correspond to different values of the base. This is why in Definition 3.2.9, we gencrically chose the base to be $1 / 2$ and call it the absolute value at $P$.

Lemma 3.2.11 (Strict Triangle Inequality) ([19] p.5) Let $\|$ be an absolute value of $F$ and let $a, b \in F$ be such that $|a| \neq|b|$.Then $|a+b|=\max \{|a|,|b|\}$.

Proof. Since $|a| \neq|b|$ we will assume without loss of generality that $|a|<|b|$. Suppose that $|a+b| \neq \max \{|a|,|b|\}$, and so $|a+b|<|b|$. We obtain that

$$
|b|=|(b+a)-(a)| \leq \max \{|a+b|,|a|\}<|b|
$$

a contradiction.

Proposition 3.2.12 Let $F$ be a function field. Then there is a one-to-one correspondence between the places of $F$ and the absolute values of $F$ up to equivalence.

Proof. To each place $P \in \mathcal{P}_{F}$ there is a discrete valuation $v_{P}$. From each discrete valuation we may define $\|$ as above. Conversely, to each absolute value of $\|$ we may define each of the following

$$
\begin{aligned}
\mathcal{O}_{P} & =\{z \in F|| z \mid \leq 1\} \\
P & =\{z \in F|| z \mid<1\} .
\end{aligned}
$$

From Definition 3.2.8 we see that an absolute value is a group homomorphism into $\mathbb{R}^{\times}$and thus $\left|z^{-1}\right|=|z|^{-1}$. Again we see that either $z$ or $z^{-1}$ is an element of $\mathcal{O}_{P}$. So $\mathcal{O}_{P}$ is indeed a valuation ring. Suppose $z \in \mathcal{O}^{*}$ then $z, z^{-1} \in \mathcal{O}$ and thus $|z| \leq 1$ 
and $\left|z^{-1}\right| \leq 1$. Combining these equations we obtain that $1 \leq|z| \leq 1$, i.e. $|z|=1$. So all units in $\mathcal{O}$ have absolute value 1 , and thus $P$ is the set of non-units which we know by Proposition 3.1.7 to be the maximal ideal of $\mathcal{O}$ and hence a place. One may easily check that equivalent absolute values define the same place. Therefore, to each equivalence class of absolute values we may associate a place and vice versa.

Remark 3.2.13 Combining Corollary 3.2.7 and Proposition 3.2.12, we have shown that valuations, and equivalence classes of absolute values are both in one-to-one correspondence with places. So by transitivity, valuations and equivalence classes of absolute values are in one-to-one correspondence with each other.

\subsubsection{Completions}

As we have said, absolute values give us a measure of "distance" between elements. This affords us the ability to discuss the convergence of sequences of elements. We begin by recalling Cauchy sequences.

Definition 3.2.14 ([7] p.456) Let $\left\{z_{n}\right\}$ be a sequence of elements in $F$ and suppose that $F$ is equipped with an absolute value $\|$. The sequence $\left\{z_{n}\right\}$ is called a Cauchy sequence if for any $0<\epsilon \in \mathbb{R}$, there exists a positive integer $N$ such that $\left|z_{a}-z_{b}\right|<\epsilon$ for all $a, b \geq N$.

Remark 3.2.15 ([7] p.456) Without loss of generality let $b>a$, then since

$$
\begin{aligned}
\left|z_{a}-z_{b}\right| & =\left|\left(z_{a}-z_{a+1}\right)+\left(z_{a+1}-z_{a+2}\right)+\ldots+\left(z_{b+1}-z_{b}\right)\right| \\
& \leq \max \left\{\left|z_{a}-z_{a+1}\right|,\left|z_{a+1}-z_{a+2}\right|, \ldots\left|z_{b+1}-z_{b}\right|\right\}
\end{aligned}
$$

we have that sequence $\left\{z_{n}\right\}$ is Cauchy if and only if for any $\epsilon>0$ there exists an $N$ such that $\left|z_{n}-z_{n+1}\right|<\epsilon$ for all $n>N$.

Example 3.2.16 Let $F$ be a function field and let $P$ be a place in $F$. Let $\left\{z_{n}\right\}=\left(z_{0}, z_{1}, \ldots\right)$ be a sequence in $F$ i.e. $z_{i} \in F$ for all $i=0,1, \ldots$ If we consider the absolute value $\|_{P}$ a measure of "distance" then we wish to examine when 
the "distance" between successive elements is decreasing. By the Strict Triangle inequality,

$$
\left|z_{i}-z_{i+1}\right|_{P} \leq \max \left\{\left|z_{i}\right|_{P},\left|z_{i+1}\right|_{P}\right\}
$$

One possible case is that the absolute values of the elements are strictly decreasing. i.e. $\left|z_{i}\right|_{P}>\left|z_{i+1}\right|_{P}$. In this case our sequence would be Cauchy.

Another case is that $z_{i}=z_{i+1}$ for all $i>N$ for some $N>0$. Then $\left|z_{i}-z_{i+1}\right|_{P}=0$ for all $i>N$. In this case our sequence would also be Cauchy.

There is a particular, perhaps less obvious, case we also wish to consider. Let $t$ be a local parameter of $P$. Since $z \in F$, by Theorem 3.1.13 there exists some $n+1 \in \mathbb{Z}$ such that $z=u_{1} t^{n+1}$ for some unit $u_{1}$. Define the sequence $\left\{z_{i}\right\}$ by $z_{1}=z$, $z_{i+1}=z_{i}+u_{i+1} t^{n+i+1}$ where $u_{i+1}$ are units of $\mathcal{O}_{P}$. Hence, a general term of the sequence, $z_{m}$, is

$$
z_{m}=u_{1} t^{n+1}+u_{2} t^{n+2}+\ldots+u_{m} t^{n+m}=\sum_{i=1}^{m} u_{i} t^{n+i}
$$

Now, let's examine the absolute value of successive terms.

$$
\begin{aligned}
\left|z_{m}-z_{m+1}\right|_{P} & =\left|\sum_{i=1}^{m} u_{i} t^{n+i}-\sum_{i=1}^{m+1} u_{i} t^{n+i}\right|_{P} \\
& =\left|u_{m+1} t^{n+m+1}\right|_{P} \\
& =\left.\left|u_{m+1}\right| t^{n+m+1}\right|_{P} \\
& =1 \cdot(1 / 2)^{n+m+1} \\
& =(1 / 2)^{n}(1 / 2)^{m+1}
\end{aligned}
$$

We notice that $\left|z_{m}-z_{m+1}\right|=(1 / 2)^{n}(1 / 2)^{m+1} \rightarrow 0$ as $m \rightarrow \infty$. Therefore, in this case our sequence is again Cauchy. There are two interesting features of this particular sequence. 
1. Notice that $\left|z_{m}\right|=|z|$ for all $m \geq 0$. Indeed, by the Strict Triangle Inequality

$$
\left|z_{m}\right|=\max _{1 \leq i \leq m}\left\{\left|u_{i} t^{n+i}\right|\right\}=\left|t^{n+1}\right|
$$

Yet, despite this fact, the sequence is Cauchy.

2. The element to which the sequence converges may not be in $F$. Indeed, by inspection, the limit of this sequence is

$$
\hat{z}=\sum_{i=1}^{\infty} u_{i} t^{n+i}
$$

which may not be an element on $F$.

Our goal in the remainder of this section is to introduce the completion of $F$, a field in which the element $\hat{z}$ of Equation (3.8) is defined. With this in mind, we give the definition of convergence.

Definition 3.2.17 ([7] p.456) Let $\left\{z_{n}\right\}$ be a sequence of elements in $F$ with an absolute value $\|$. We say that the sequence $\left\{z_{n}\right\}$ converges to $z$ if for any $0<\epsilon \in \mathbb{R}$, there exists a positive integer $N$ such that $\left|z_{a}-z\right|<\epsilon$ for all $a \geq N$.

In general, it is not true that every Cauchy sequence converges to an element of $F$. Hence we have the following definitions.

Definition 3.2.18 ([19] p.140) A field $F$ is said to be complete relative to $\|$ if every Cauchy sequence of elements in $F$ converges to an element in $F$.

Definition 3.2.19 ([19] p.141) Let $F$ be a field with an absolute value $\|_{P}$. A completion of $F$ with respect to $\|_{P}$ is a field $\hat{F}$ with absolute value $\|_{\hat{P}}$ with the following properties.

1. $F \subseteq \hat{F}$ and $\|_{P}$ is the restriction of $\|_{\hat{P}}$ to $F$.

2. $\hat{F}$ is complete with respect to $\|_{\hat{P}}$. 
3. $F$ is dense in $\hat{F}$, i.e for any $z \in \hat{F}$ there exists a sequence $\left\{x_{n}\right\}$ in $F$ that converges to $z$.

This invites the question of whether or not we may find a completion of a function field $F$ for each absolute value on $F$. This is answered by the following proposition.

Proposition 3.2.20 ([19] p.141) For any field $F$ with absolute value $\|_{P}$ there exists a completion $\hat{F}$ with absolute value $\|_{\hat{P}}$. Moreover, the completion is unique up to isomorphism.

We give only a sketch of the proof to demonstate the construction and properties of a completion. For further details see [7] p.458.

Sketch of Proof. We begin by considering the set

$$
R=\left\{\left\{z_{n}\right\} \mid\left\{z_{n}\right\} \text { is a Cauchy sequence in } F\right\} .
$$

We may define an addition and a multiplication on $R$ as

$$
\left\{z_{n}\right\}+\left\{y_{n}\right\}=\left\{z_{n}+y_{n}\right\} \quad\left\{z_{n}\right\} \cdot\left\{y_{n}\right\}=\left\{z_{n} y_{n}\right\}
$$

to view $R$ as a ring. Define the set $I$ such that

$$
I=\left\{\left\{z_{n}\right\} \mid\left\{z_{n}\right\} \text { converges to } 0\right\} .
$$

One may check that $I$ is an ideal of $R$, and subsequently that $I$ is a maximal ideal of $R$. Therefore the quotient ring

$$
\widehat{F}=R / I
$$

is a field. Define a map $\rho: F \rightarrow \widehat{F}$ by $\rho(z)=(z, z, z, \ldots)+I$. Clearly $\rho$ is an embedding and we consider $F$ a subfield of $\hat{F}$ via this embedding.

We now construct an absolute value $\|_{\hat{P}}$ on $\hat{F}$ as follows. If $\left\{z_{n}\right\}$ is a Cauchy in $F$ 
then either

$$
\lim _{n \rightarrow \infty}\left|z_{n}\right|_{P}=0
$$

or

there exists an $N>0$ such that $\left|z_{n}\right|_{P}=\left|z_{m}\right|_{P}$ for all $m, n>N$.

In either case, the limit $\lim _{n \rightarrow \infty}\left|z_{n}\right|_{P}$ exists and is a positive real number. Consequently, if $\left\{z_{n}\right\}-\left\{y_{n}\right\} \in I$ then we have that $\lim _{n \rightarrow \infty}\left|z_{n}\right|_{P}=\lim _{n \rightarrow \infty}\left|y_{n}\right|_{P}$. Therefore we have a well defined function $\|_{\hat{P}}: \hat{F} \rightarrow \mathbb{R}$ by

$$
\left|\left\{z_{n}\right\}+I\right|_{\hat{P}}=\lim _{n \rightarrow \infty}\left|z_{n}\right|_{P}
$$

Using the properties of $\|_{P}$ one may check that this is indeed an absolute value. Clearly using the embedding $\rho$, we have that $|z|_{\hat{P}}=|z|_{P}$ for all $z \in F$.

For a proof that $\hat{F}$ is complete, unique up to isomorphism, and that $F$ is dense in $\hat{F}$, see [19] p.142.

We will often make the common abuse of notation writing $\|_{P}$ for $\|_{\hat{P}}$. We will also typically drop the notation of the quotient ring and write $\left\{z_{n}\right\}$ for $\left\{z_{n}\right\}+I$.

If absolute values $\|_{1}$ and $\|_{2}$ on a field $F$ are equivalent, then clearly a sequence is Cauchy with respect to $\|_{1}$ if and only if it is Cauchy with respect to $\|_{2}$. As a result, equivalent absolute values yield isomorphic completions. In this sense, there is a unique completion (up to isomorphism) with respect to an absolute value (up to equivalence). This creates a one-to-one correspondence between the two.

In Remark 3.2.13, we already discussed that absolute values are in one-to-one correspondence to both discrete valuations on $F$ and places of $F$. So in fact, we have shown that to each field $F$ we have the following diagram.

$$
P \in \mathcal{P}_{F} \stackrel{1-1}{\longleftrightarrow} v_{P} \stackrel{1-1}{\longleftrightarrow} \|_{P} \stackrel{1-1}{\longleftrightarrow} \hat{F}
$$

Likewise, for any other function field $F^{\prime} \supset F$ there is the same 1-1 correspondence 
between the places, discrete valuations, absolute values and completions of $F^{\prime}$.

It is often convenient to represent elements of $\hat{F}$ in a more "usable" manner, namely the manner in which we expressed $\hat{z}$ in Equation (3.8).

Proposition 3.2.21 ([19] p.143) Let $P$ be a place of a function field $F$ with $t$ a local parameter at $P$ (Definition 3.1.15). Let $z \in \hat{F}$. Then $z$ has a representation in the form

$$
z=\sum_{i=m}^{\infty} a_{i} t^{i} \quad \text { with } m \in \mathbb{Z} \quad \text { and } a_{i} \in \mathcal{O}_{P}^{*}
$$

Secondly, $|z|_{\hat{P}}=\left|t^{j}\right|_{P}$ where $j=\min \left\{i \mid a_{i} \neq 0\right\}$.

Proof. Recall that we will be writing $\|_{P}$ for $\|_{\hat{P}}$. Choose an $n$ such that $\left|t^{n}\right|_{P}>|z|_{P}$. Then since $F$ is dense in $\hat{F}$ there exists an element $y \in F$ such that $|z-y|_{P}<\left|t^{n}\right|_{P}$. We will prove by contradiction that $|y|_{P} \leq\left|t^{n}\right|_{P}$. Assume that $|y|_{P}>\left|t^{n}\right|_{P}$. Then $|y|_{P}>\left|t^{n}\right|_{P}>|z|_{P}$ so by the Strict Triangle Inequality, we have that

$$
|z-y|_{P}=\max \left\{|z|_{P},|y|_{P}\right\}=|y|_{P}>\left|t^{n}\right|_{P}
$$

Which contradicts the choice of $y$. Therefore $|y|_{P} \leq\left|t^{n}\right|_{P}$ and consequently $\left|y t^{-n}\right|_{P} \leq$ 1. i.e. $y t^{-n} \in \mathcal{O}_{P}$. If $y t^{-n} \in \mathcal{O}_{P}^{*}$ then $y t^{-n}+P=u+P$ for some unit $u \in \mathcal{O}_{P}$. Set $a_{n}=0$ if $y t^{-n} \in P$ or $u$ otherwise. Then $y t^{-n}-a_{n} \in P$ and thus $\left|y t^{-n}-a_{n}\right|_{P}<1$ and thus $\left|y-a_{n} t^{n}\right|_{P}<\left|t^{n}\right|_{P}$. Therefore by the Strict Triangle Inequality,

$$
\left|z-a_{n} t^{n}\right|_{P}=\left|(z-y)+\left(y-a_{n} t^{n}\right)\right|_{P} \leq \max \left\{|(z-y)|_{P},\left|\left(y-a_{n} t^{n}\right)\right|_{P}\right\}<\left|t^{n}\right|_{P}=(1 / 2)^{n} .
$$

Repeating this process with $z-a_{n} t^{n}$ instead of $z$, we find $a_{n+1} \in \mathcal{O}_{P}^{*}$ such that

$$
\left|z-a_{n} t^{n}-a_{n+1} t^{n+1}\right|_{P} \leq\left|t^{n+1}\right|_{P}=(1 / 2)^{n+1} .
$$


Repeating again $m$ times we have that

$$
\left|z-\sum_{i=n}^{m} a_{i} t^{i}\right|_{P} \leq(1 / 2)^{n+m}
$$

for all $m>n$. Consequently, repeating this process indefinitely produces a sequence $\left\{a_{i}\right\}_{i=1}^{\infty}$ such that

$$
\left|z-\sum_{i=n}^{\infty} a_{i} t^{i}\right|_{P}=0
$$

i.e.

$$
z=\sum_{i=n}^{\infty} a_{i} t^{i}
$$

as desired.

We typically take $m=\min \left\{i \mid a_{i} \neq 0\right\}$ making the expression for $z$

$$
z=\sum_{i=m}^{\infty} a_{i} t^{i} \quad \text { with } m \in \mathbb{Z} \quad \text { and } a_{i} \in \mathcal{O}_{P}^{*}
$$

where $a_{m} \neq 0$. Consequently $|z|_{P}=\left|t^{m}\right|_{P}$.

Recall from Definition 3.1.17 that the degree of a place is the degree of the exten$\operatorname{sion}\left[F_{P}: K\right]$.

Corollary 3.2.22 Let $P$ be a place of $K(x)$ of degree one. Any $z \in \hat{F}$ has a unique representation in the form

$$
z=\sum_{i=m}^{\infty} a_{i} t^{i} \quad \text { with } m \in \mathbb{Z}
$$

where $a_{i} \in K$ and $a_{m} \neq 0$.

Proof. Since $\operatorname{deg}(P)=1, \mathcal{O}_{P}^{*} \cong \mathcal{O}_{P} / P \cong K$, the $a_{i}$ 's in the construction of the proof of Proposition 3.2.21 may be uniquely chosen as elements of $K$. 
Corollary 3.2.23 Let $P$ be a place of $K(x)$ of degree $d$. Then $z$ has a unique representation in the form

$$
z=\sum_{i=m}^{\infty} a_{i}(x) t^{i} \quad \text { with } m \in \mathbb{Z}
$$

where $a_{i}(x) \in K[x], a_{m} \neq 0$, and $\operatorname{deg}\left(a_{i}(x)\right)<d$.

Proof. From Example 3.1.19, $F_{P} \cong K[x] /(p(x))$ thus the $a_{i}$ 's in the construction of the proof of Proposition 3.2.21 may be uniquely chosen as elements of $K[x]$ whose degree is less than $d$.

Since $m$ may be negative, the expression in Equation (3.10) is typically called the Laurent series in $t$ of $z$ over $K$. We will use the term Laurent series in $t$ of $z$ more generally to describe any expression for $z$ of the type described in Proposition 3.2.21.

Definition 3.2.24 Let $P$ be a place of a function field $F$ with $t$ a local parameter at $P$ (Definition 3.1.15). Let $z \in \hat{F}$. By Proposition 3.2.21, we may express $z$ in a Laurent series in $t$ as

$$
z=\sum_{i=m}^{\infty} a_{i} t^{i} \quad \text { with } m \in \mathbb{Z} \quad \text { and } a_{i} \in \mathcal{O}_{P}^{*}
$$

Define $v_{\hat{P}}: \hat{F} \rightarrow \mathbb{Z} \cup \infty$ as $v_{P}(0)=\infty$ and $v_{\hat{P}}=j$ where $j=\min \left\{i \mid a_{i} \neq 0\right\}$ for $z \neq 0$.

$v_{\hat{P}}$ is the valuation corresponding to $\|_{\hat{P}}$ as described in Proposition 3.2.21.

Since $\|_{\hat{P}}$ is an absolute value on $\hat{F}$, it defines a valuation ring, a place, and residue class field of $\hat{F}$ in a similar manner as Proposition 3.2.12. We will denote these as $\hat{\mathcal{O}}_{P}, \hat{P}$, and $\hat{F}_{P}$ respectively. As in Proposition 3.2 .12 , we have that

$$
\begin{aligned}
\hat{\mathcal{O}}_{P} & =\left\{\left.\hat{z} \in \hat{F}|| z\right|_{\hat{P}} \leq 1\right\}, \\
\hat{P} & =\left\{\hat{z} \in \hat{F} \|\left. z\right|_{\hat{P}}<1\right\}, \\
\hat{\mathcal{O}}_{P}^{*} & =\left\{\left.\hat{z} \in \hat{F}|| z\right|_{\hat{P}}=1\right\} .
\end{aligned}
$$


In addition, since $|z|_{\hat{P}}=|z|_{P}$ for all $z \in F$ we have that

$$
\mathcal{O}_{P}=\hat{\mathcal{O}}_{P} \cap F \quad \text { and } \quad P=\hat{P} \cap F .
$$

\subsubsection{The Completions of $K(x)$}

We will now concretely describe the completions of each place in $K(x)$. We partition the places of $K(x)$ into three classes and examine each class separately.

\section{Finite Places of Degree One}

Let $P_{a}$ be the place corresponding to the irreducible polynomial $x-a \in K[x]$ for some $a \in K$ (Theorem 3.1.20). We saw in Example 3.1.16 that $x-a$ is a local parameter at $P_{a}$. Therefore, by Corollary 3.2 .22 every element $\hat{z} \in \hat{F}$ may uniquely be expressed as

$$
z=\sum_{i=m}^{\infty} a_{i}(x-a)^{i} \quad \text { with } m \in \mathbb{Z} \quad \text { and } a_{i} \in K, a_{m} \neq 0
$$

with $|z|_{\hat{P}_{a}}=|x-a|_{P_{a}}^{m}=(1 / 2)^{m}$. As a result,

$$
\begin{aligned}
\hat{\mathcal{O}}_{P} & =\left\{\hat{z} \in \hat{F} \mid z=\sum_{i=m}^{\infty} a_{i}(x-a)^{i} \text { with } m \geq 0\right\}, \\
\hat{P} & =\left\{\hat{z} \in \hat{F} \mid z=\sum_{i=m}^{\infty} a_{i}(x-a)^{i} \text { with } m>0\right\}, \\
\hat{\mathcal{O}}_{P}^{*} & =\left\{\hat{z} \in \hat{F} \mid z=\sum_{i=m}^{\infty} a_{i}(x-a)^{i} \text { with } m=0\right\} .
\end{aligned}
$$

\section{The Places at Infinity}

Let $P_{\infty}$ be the infinite place of $K(x)$ (Theorem 3.1.20). We saw in Example 3.1.16 that $1 / x$ is a local parameter at $P_{\infty}$. Therefore, by Corollary 3.2 .22 every element 
$\hat{z} \in \hat{F}$ may uniquely be expressed as

$$
z=\sum_{i=m}^{\infty} a_{i}(1 / x)^{i}=\sum_{i=m}^{\infty} a_{i} x^{-i}
$$

with $m \in \mathbb{Z}, a_{i} \in K$, and $a_{m} \neq 0$ and $|z|_{\hat{P}_{\infty}}=|1 / x|_{P_{\infty}}^{m}=(1 / 2)^{m}$. As a result,

$$
\begin{aligned}
\hat{\mathcal{O}}_{P} & =\left\{\hat{z} \in \hat{F} \mid z=\sum_{i=m}^{\infty} a_{i} x^{-i} \text { with } m \geq 0\right\}, \\
\hat{P} & =\left\{\hat{z} \in \hat{F} \mid z=\sum_{i=m}^{\infty} a_{i} x^{-i} \text { with } m>0\right\}, \\
\hat{\mathcal{O}}_{P}^{*} & =\left\{\hat{z} \in \hat{F} \mid z=\sum_{i=m}^{\infty} a_{i} x^{-i} \text { with } m=0\right\} .
\end{aligned}
$$

\section{Finite Places of Degree Greater Than One}

Let $P_{p(x)}$ be the place corresponding to the irreducible polynomial $p(x)$ (Theorem 3.1.20). We saw in Example 3.1.16 that $p(x)$ is a local parameter at $P_{p(x)}$. Therefore, by Corollary 3.2.23 every element $\hat{z} \in \hat{F}$ may uniquely be expressed as

$$
z=\sum_{i=m}^{\infty} a_{i}(x) p(x)^{i}
$$

with $m \in \mathbb{Z}, a_{i}(x) \in K[x], a_{m}(x) \neq 0$ and $\operatorname{deg}\left(a_{m}(x)\right)<\operatorname{deg}(p(x))$. Consequently $|z|_{\hat{P}_{p}(x)}=|p(x)|_{P_{a}}^{m}=(1 / 2)^{m}$. As a result

$$
\begin{gathered}
\hat{\mathcal{O}}_{P}=\left\{\hat{z} \in \hat{F} \mid z=\sum_{i=m}^{\infty} a_{i}(x)(p(x))^{i} \text { with } m \geq 0\right\}, \\
\hat{P}=\left\{\hat{z} \in \hat{F} \mid z=\sum_{i=m}^{\infty} a_{i}(x)(p(x))^{i} \text { with } m>0\right\}, \\
\hat{\mathcal{O}}_{P}^{*}=\left\{\hat{z} \in \hat{F} \mid z=\sum_{i=m}^{\infty} a_{i}(x)(p(x))^{i} \text { with } m=0\right\} .
\end{gathered}
$$

There seems to be an equivalence between the degree of a place $\left[\mathcal{O}_{P} / P: K\right]$ and 
the degree of the corresponding place in the completion $\left[\mathcal{O}_{\hat{P}} / \hat{P}: K\right]$. Indeed, this is the case and we will prove this in general later on. For now, let $P$ be a place of $K(x)$ and let $t$ be a local parameter at $P$. Let $\hat{F}$ denote the completion of $K(x)$ with respect to $\|_{P}$. Let $\hat{z} \in \hat{F}$. By Proposition 3.2.21 we have that

$$
z=\sum_{i=0}^{\infty} a_{i}(x) t^{i}
$$

where $a_{i}(x) \in \mathcal{O}_{P}^{*}$. Now applying the quotient map modulo $\hat{P}$, this equation becomes

$$
z+\hat{P}=\sum_{i=0}^{\infty} a_{i}(x) t^{i}+\hat{P}
$$

Since

$$
z_{1}=\sum_{i=1}^{\infty} a_{i}(x) t^{i} \in \hat{P}
$$

we have that

$$
z+\hat{P}=a_{0}(x)+z_{1}+\hat{P}=a_{0}(x)+\hat{P} .
$$

Consider the map $\tau: \mathcal{O}_{P} \rightarrow \hat{F}_{P}$ where $\tau(a(x))=a(x)+\hat{P}$. Then by the above, $\tau$ is onto. In addition, the kernel of $\tau$ is the set of $a(x) \in K(x)$ such that $a(x) \in \hat{P}$. This is equivalent to saying that $|a(x)|_{\hat{P}}<1$, i.e. $|a(x)|_{P}<1$ or equivalently $a(x) \in P$. Therefore, by the First Isomorphism Theorem of rings, $F_{P} \cong \hat{F}_{P}$, and thus $\operatorname{deg}(P)=$ $\operatorname{deg}(\hat{P})$.

\subsection{Extensions of Function Fields}

By Definition 3.1.2, a function field $F^{\prime}$ is a finite extension of $K(x)$. In this section we consider function fields in this manner, namely as extension fields. Doing so will allow us to compare the places, absolute values and valuations of $F^{\prime}$ with those of $K(x)$. We define two important properties; the ramification index and the relative degree. The ramification index is a positive integer $e$ which relates the valuation of 
the place $P \subset K(x)$ with that of the place $P^{\prime} \in K(x, y)$ where $P \subset P^{\prime}$. The relative degree $f$ is a positive integer that relates the residue fields $\mathcal{O}_{P} / P$ and $\mathcal{O}_{P^{\prime}} / P^{\prime}$ where $\mathcal{O}_{P}$ and $\mathcal{O}_{P^{\prime}}$ are the valuation rings of the places $P$ and $P^{\prime}$ respectively. The final subsection serves as a brief introduction to Galois Theory. In addition, this subsection demonstrates how Galois automorphisms effect the properties of places.

\subsubsection{Places and Extensions}

We begin with a general definition.

Definition 3.3.1 ([19] p.59) An algebraic function field $F^{\prime} / K$ is called an algebraic extension of $F / K$ if $F^{\prime} \supseteq F$ is an algebraic extension. The algebraic extension $F^{\prime} / K$ is called a finite extension if $\left[F^{\prime}: F\right]<\infty$.

As we are mainly be concerned with quadratic extensions, we examine their structure as a field extension.

Lemma 3.3.2 Let $F^{\prime}$ be a quadratic extension of $K(x)$. Then there exists an element $y \in F^{\prime}$ such that $F^{\prime}=K(x, y)$ and

$$
y^{2}=\varphi(x)=\prod_{i=1}^{\eta} \rho_{i}(x) \in K[x]
$$

where $\rho_{1}(x), \ldots, \rho(x)_{\eta}$ are distinct irreducible polynomials in $K[x]$.

Proof. $F^{\prime}$ being quadratic over $K(x)$ implies that there exists an element $y^{\prime} \in F^{\prime}$ such that $y \notin K(x)$ and $y^{\prime 2}+g(x) y^{\prime}=h(x)$ for some $g(x), h(x) \in K(x)$. Since $\operatorname{char}(K) \neq 2$ we may complete the square, which yields

$$
\left(y^{\prime}-1 / 2 \cdot g(x)\right)^{2}=h(x)+1 / 4 \cdot g(x)^{2}
$$


Set $y^{\prime \prime}:=y^{\prime}-1 / 2 \cdot g(x)$. Substituting into (3.12) we have that

$$
y^{\prime \prime 2}=\prod_{i=1}^{m} \rho_{i}(x)^{k_{i}}
$$

where $k_{1}, \ldots, k_{\eta}$ are positive integers and $\rho_{1}(x), \ldots, \rho_{m}(x)$ are distinct irreducible polynomials in $K[x]$. For $i=1, . ., m$ we may write $k_{i}=2 q_{i}+r_{i}$ for some $q_{i}, r_{i} \in \mathbb{Z}$, where $q_{i} \geq 0, r_{i}=0$ or 1 . Set $y:=y^{\prime \prime} \cdot \prod_{i=1}^{m} \rho_{i}(x)^{-q_{i}}$ then from (3.13) we obtain that

$$
\left(y \cdot \prod_{i=1}^{m} \rho_{i}(x)^{q_{i}}\right)^{2}=\prod_{i=1}^{m} \rho_{i}(x)^{k_{i}} \Rightarrow y^{2}=\prod_{i=1}^{m} \rho_{i}(x)^{r_{i}} .
$$

So after re-indexing to remove those $\rho_{i}(x)$ whose exponent is 0 , we have that

$$
y^{2}=\prod_{i=1}^{\eta} \rho_{i}(x)
$$

as required. Furthermore, since $y^{\prime}$ is not in $K(x), y \notin K(x)$. This implies that $F^{\prime} \supseteq K(x, y) \supset K(x)$. By the tower property of fields we have that

$$
\left[F^{\prime}: K(x, y)\right]=\frac{\left[F^{\prime}: K(x)\right]}{[K(x, y): K(x)]}=\frac{2}{2}=1
$$

Therefore $F^{\prime}=K(x, y)$.

Remark 3.3.3 Henceforth, any quadratic extension $K(x, y)$ of $K(x)$ will be assumed to be as in the conclusions of Lemma 3.3.2.

Now that we have a convenient way to express a quadratic extension, we may begin to examine some the places of a quadratic extension field. We do not yet have the tools to describe all the places of a quadratic extension, but we will give a few examples to aid in the understanding of this section.

Example 3.3.4 As a specific case of Example 3.1.22, we will use the evaluation map to describe some places of a quadratic extension. Let $K(x, y)$ be a quadratic 
extension of $K(x)$. Then according to Lemma 3.3.2, we may assume that $y^{2}=\varphi(x)$. Let $c(x, y)=y^{2}-\varphi(x)$. We will assume that there exists some $p_{\mathbf{1}}, p_{2} \in K$ such that $\varphi\left(p_{1}\right)=p_{2}^{2}$, i.e. $c\left(p_{1}, p_{2}\right)=0$. Then just as we did in Section 2.3 we will define the place $P$ and the ring $\mathcal{O}_{P}$ as

$$
\begin{aligned}
\mathcal{O}_{P} & =\left\{\frac{a(x, y)}{b(x, y)} \in K(x, y) \mid b\left(p_{1}, p_{2}\right) \neq 0\right\} \\
P & =\left\{\frac{a(x, y)}{b(x, y)} \in K(x, y) \mid a\left(p_{1}, p_{2}\right)=0, b\left(p_{1}, p_{2}\right) \neq 0\right\} .
\end{aligned}
$$

Then just as in Proposition 2.3.16, $\mathcal{O}_{P}$ is a valuation ring. Since $P$ is the kernel of the evaluation homomorphism $\mathcal{O}_{P} \rightarrow K, \mathcal{O}_{P} / P \cong K$, and hence $P$ is a maximal ideal. So by Definition 3.1.8 it is a place of $K(x, y)$.

Upon examination, we may see that the place $P$ above contains the ideal $\left(x-p_{\mathbf{I}}\right)$ of $\mathcal{O}_{P}$. We also notice that $\mathcal{O}_{x-p_{1}} \subset \mathcal{O}_{P}$. So the place $P_{x-p_{1}}$ of $K(x)$ is contained in the place $P$ of $K(x, y)$ described above. We give a more formal definition to this property.

Definition 3.3.5 ([19] p.60) Let $F^{\prime} / F$ be function fields over $K$. A place $P^{\prime} \in \mathcal{P}_{F^{\prime}}$ lies over a place $P \in \mathcal{P}_{F^{\prime}}$ if $P \subseteq P^{\prime}$. We also say that $P$ lies under $P^{\prime}$ and we denote this by $P^{\prime} \mid P$.

Proposition 3.3.6 ([19] p.60) Let $F^{\prime} / F$ be function fields over $K$. Let $P$ be a place of $F$, and let $P^{\prime}$ be a place of $F^{\prime}$ with valuation rings $\mathcal{O}_{P}$ and $\mathcal{O}_{P^{\prime}}$, and discrete valuations $v_{P}$ and $v_{P^{\prime}}$ respectively. Then the following are equivalent.

1. $P^{\prime} \mid P$

2. $\mathcal{O}_{P} \subseteq \mathcal{O}_{P^{\prime}}$

Moreover, if $P^{\prime} \mid P$ then

$$
P=P^{\prime} \cap F \quad \text { and } \quad \mathcal{O}_{P}=\mathcal{O}_{P^{\prime}} \cap F
$$


Proof. $(1) \Rightarrow(2)$ : Suppose that $P^{\prime} \mid P$ but $\mathcal{O}_{P} \nsubseteq \mathcal{O}_{P^{\prime}}$. Then there exists some $u \in \mathcal{O}_{P}$ such that $u \notin \mathcal{O}_{P^{\prime}}$. By Theorem 3.2.3 is implies that $v_{P}(u) \geq 0$ and $v_{P^{\prime}}(u)<0$. Since $P \subset P^{\prime}$, and $v_{P^{\prime}}(u)>0$ implies that $u \in P$, we conclude that $v_{P}(u)=0$. Let $t \in P$ be a local parameter at $P$ as in Definition 3.1.15. By Theorem 3.2.3 $v_{P}(t)=1$ and as such $t \in P^{\prime}$. Define $r=v_{P^{\prime}}(t)>0$. We consider the element $u^{r} t$ and see that

$$
\begin{gathered}
v_{P}\left(u^{r} t\right)=r \cdot v_{P}(u)+v_{P}(t)=1 \quad \text { and } \\
v_{P^{\prime}}\left(u^{r} t\right)=r \cdot v_{P^{\prime}}(u)+v_{P^{\prime}}(t) \leq-r+r=0 .
\end{gathered}
$$

Thus $u^{r} t \in P$ and $u^{r} t \notin P^{\prime}$, a contradiction to $P \subseteq P^{\prime}$.

$(2) \Rightarrow(1):$ Suppose $\mathcal{O}_{P} \subseteq \mathcal{O}_{P^{\prime}}$. Let $z \in P$ then by Remark 3.1.12, $z^{-1} \notin \mathcal{O}_{P}$. Therefore, by equation (3.14), which we will prove below, $z^{-1}$ is not in $\mathcal{O}_{P^{\prime}}$. This implies that $\left(z^{-1}\right)^{-1}=z \in P^{\prime}$ and thus $P \subseteq P^{\prime}$.

Proof of equation (3.14): Suppose that $P^{\prime} \mid P$. Then by the proof $(1) \Rightarrow(2), \mathcal{O}_{P} \subseteq \mathcal{O}_{P^{\prime}}$. Hence, we need only prove that

$$
\mathcal{O}_{P} \subseteq \mathcal{O}_{P^{\prime}} \Rightarrow \mathcal{O}_{P}=F \cap \mathcal{O}_{P^{\prime}}
$$

Clearly, $F \cap \mathcal{O}_{P^{\prime}}$ is a subring of $F$ with $\mathcal{O}_{P} \subseteq F \cap \mathcal{O}_{P^{\prime}}$. From Lemma 3.1.21 we know that $\mathcal{O}_{P}$ is a maximal subring of $F$. Consequently, either $\mathcal{O}_{P}=F \cap \mathcal{O}_{P^{\prime}}$ or $F=F \cap \mathcal{O}_{P^{\prime}}$. Assume that $F=F \cap \mathcal{O}_{P^{\prime}}$. This implies that $F \subseteq \mathcal{O}_{P^{\prime}}$. Choose and element $z \in F^{\prime} \backslash \mathcal{O}_{P^{\prime}}$. Since $F^{\prime} / F$ is an algebraic extension, there exists an equation

$$
z^{n}+c_{n-1} z^{n-1}+\ldots+c_{1} z+c_{0}=0
$$

with $c_{i} \in F$ for $i=0, \ldots, n-1$. We have that $v_{P^{\prime}}\left(z^{n}\right)=n \cdot v_{P^{\prime}}(z)<0$ as $z \notin \mathcal{O}_{P^{\prime}}$. So by Definition 3.2.1,

$$
v_{P^{\prime}}\left(c_{i} z^{i}\right)=v_{P^{\prime}}\left(c_{i}\right)+v_{P^{\prime}}\left(z^{i}\right) \geq 0+v_{P^{\prime}}\left(z^{i}\right)>v_{P^{\prime}}\left(z^{n}\right) \quad \text { for } \quad i=0,1, \ldots, n-1 .
$$


By the Strict Triangle Inequality (Lemma3.2.2), we have that

$$
v_{P^{\prime}}\left(z^{n}+c_{n-1} z^{n-1}+\ldots+c_{1} z+c_{0}\right)=n \cdot v_{P^{\prime}}(z) \neq v_{P^{\prime}}(0)=0 .
$$

This contradicts equation (3.16), which implies that $F \cap \mathcal{O}_{P^{\prime}} \neq F$, and proves equation (3.15). Intersecting both sides of the equation on the right in (3.15) with $P^{\prime}$ we see that

$$
\begin{aligned}
\mathcal{O}_{P} \subseteq \mathcal{O}_{P^{\prime}} & \Rightarrow \mathcal{O}_{P} \cap P^{\prime}=F \cap \mathcal{O}_{P^{\prime}} \cap P^{\prime} \\
& \Rightarrow P=F \cap P^{\prime}
\end{aligned}
$$

Which proves all the claims of the proposition.

Corollary 3.3.7 ([19] p.62) Let $F^{\prime} / F$ be an extension of function fields and $P^{\prime} \in \mathcal{P}_{F^{\prime}}$. Then there exists a unique place $P \in \mathcal{P}_{F}$ that lies under $P^{\prime}$.

Proof. The unique place lying under $P^{\prime}$ is $P=P^{\prime} \cap F$. This intersection is clearly a place of $F$ as long as it is nonempty by Definition 3.1.8. We need only therefore show that there exists an element $z \in F$ such that $v_{P}^{\prime}(z) \neq 0$, because this implies that $z$ or $z^{-1}$ as in $P$. Assume this is false. Choose $t \in F^{\prime}$ such that $v_{P^{\prime}}(t)>0$. Then since $F^{\prime} / F$ is algebraic, there exists an equation

$$
c_{n} t^{n}+c_{n-1} t^{n-1}+\ldots+c_{1} t+c_{0}=0
$$

where $c_{i} \in F$ for $0 \leq i \leq n$. We may also assume without loss of generality that $c_{n} \neq 0$ and by factoring out a sufficiently large power of $t$ we may additionally assume that $c_{0} \neq 0$. By assumption, since $c_{0} \in K$, we have that $v_{P^{\prime}}\left(-c_{0}\right)=0$ by Definition 3.2.1. So by the Strict Triangle Inequality (Lemma 3.2.2)

$$
\begin{aligned}
v_{P^{\prime}}\left(c_{n} t^{n}+c_{n-1} t^{n-1}+\ldots+c_{1} t\right) & =v_{P^{\prime}}\left(-c_{0}\right) \\
\min _{1 \leq i \leq n}\left\{v_{P^{\prime}}\left(c_{i} t^{i}\right)\right\} & =0
\end{aligned}
$$


However $v_{P^{\prime}}\left(c_{i} t^{i}\right)=v_{P^{\prime}}\left(c_{i}\right)+i v_{P^{\prime}}(t)>0$, a contradiction.

Example 3.3.8 Continuing from Example 3.3.4, let $K(x, y)$ be a quadratic extension of $K(x)$ where $y^{2}=\varphi(x)$. Let $c(x, y)=y^{2}-\varphi(x)$. We assumed that there exists some $p_{1}, p_{2} \in K$ such that $\varphi\left(p_{1}\right)=p_{2}^{2}$, i.e. $c\left(p_{1}, p_{2}\right)=0$. We then defined the place $P$, which we will now denote as $P_{p_{1}, p_{2}}^{\prime}$, and the ring $\mathcal{O}_{P}$ which we will now write as $\mathcal{O}_{p_{1}, p_{2}}^{\prime}$, that is,

$$
\begin{aligned}
\mathcal{O}_{p_{1}, p_{2}}^{\prime} & =\left\{\frac{a(x, y)}{b(x, y)} \in K(x, y) \mid b\left(p_{1}, p_{2}\right) \neq 0\right\} \\
P_{p_{1}, p_{2}}^{\prime} & =\left\{\frac{a(x, y)}{b(x, y)} \in K(x, y) \mid a\left(p_{1}, p_{2}\right)=0, b\left(p_{1}, p_{2}\right) \neq 0\right\} .
\end{aligned}
$$

As we suspected, the place $P_{x-p_{1}}$ of $K(x)$ (Example 3.3.4), which we will denote as $P_{p_{1}}$, lies under $P_{p_{1}, p_{2}}^{\prime}$. We also know from Corollary 3.3.7, that $P_{p_{1}}$ is the unique place of $K(x)$ with this property. However, there may be another place of $K(x, y)$ that lies over $P_{p_{1}}$. If $p_{2} \neq 0$, i.e. $\varphi\left(p_{1}\right) \neq 0$, then $c\left(p_{1},-p_{2}\right)=0$ as well. Indeed $\varphi\left(p_{1}\right)-\left(-p_{2}\right)^{2}=\varphi\left(p_{1}\right)-\left(p_{2}\right)^{2}=0$. Consequently, we can define the place $P_{p_{1},-p_{2}}^{\prime}$ of $K(x, y)$ as

$$
\begin{aligned}
\mathcal{O}_{p_{1},-p_{2}}^{\prime} & =\left\{\frac{a(x, y)}{b(x, y)} \in K(x, y) \mid b\left(p_{1},-p_{2}\right) \neq 0\right\} \\
P_{p_{1},-p_{2}}^{\prime} & =\left\{\frac{a(x, y)}{b(x, y)} \in K(x, y) \mid a\left(p_{1},-p_{2}\right)=0, b\left(p_{1},-p_{2}\right) \neq 0\right\} .
\end{aligned}
$$

As before, $\mathcal{O}_{p_{1}} \subset \mathcal{O}_{p_{1},-p_{2}}^{\prime}$ and $\left(x-p_{1}\right) \in P_{p_{1},-p_{2}}^{\prime}$. Therefore, $P_{x-a}=\left(x-p_{1}\right) \mathcal{O}_{x-a} \subset$ $P_{p_{1},-p_{2}}^{\prime}$. In conclusion, both $P_{p_{1}, p_{2}}^{\prime}$ and $P_{p_{1},-p_{2}}^{\prime}$ lie over $P_{p_{1}}$. As we will see later on, these are actually the only places that lie over $P_{p_{1}}$.

\subsubsection{The Relative Degrees of Places}

Let's turn our attention to the degrees of the places $P^{\prime}$ and $P$, namely $\left[F_{P^{\prime}}: K\right]$ and $\left[F_{P}: K\right]$ respectively. Considering $\mathcal{O}_{P}$ and $\mathcal{O}_{P^{\prime}}$ as subrings of $F^{\prime}$, we apply the 
quotient map of $P^{\prime}$ and see that

$$
\begin{aligned}
\mathcal{O}_{P} \subset \mathcal{O}_{P^{\prime}} & \Rightarrow \mathcal{O}_{P}+P^{\prime} \subset \mathcal{O}_{P^{\prime}}+P^{\prime} \\
& \Rightarrow\left(\mathcal{O}_{P}+P^{\prime}\right) / P^{\prime} \subset\left(\mathcal{O}_{P^{\prime}}+P^{\prime}\right) / P^{\prime}
\end{aligned}
$$

As $\left(\mathcal{O}_{P}+P^{\prime}\right) / P^{\prime} \cong \mathcal{O}_{P} / P$, we identify $\mathcal{O}_{P} / P$ with $\left(\mathcal{O}_{P}+P^{\prime}\right) / P^{\prime}$ and conclude that

$$
\begin{aligned}
\mathcal{O}_{P} \subset \mathcal{O}_{P^{\prime}} & \Rightarrow \mathcal{O}_{P} / P \subset \mathcal{O}_{P^{\prime}} / P^{\prime} \\
& \Rightarrow F_{P} \subset F_{P^{\prime}}
\end{aligned}
$$

Since $F_{P}$ and $F_{P}^{\prime}$ are both fields, $F_{P}$ is a subfield of $F_{P^{\prime}}$. By Defintion 3.1.17, $\operatorname{deg}\left(P^{\prime}\right)=$ $\left[F_{P^{\prime}}: K\right]$, so

$$
\operatorname{deg}\left(P^{\prime}\right)=\left[F_{P^{\prime}}^{\prime}: K\right]=\left[F_{P^{\prime}}^{\prime}: F_{P}\right]\left[F_{P}: K\right]=\left[F_{P^{\prime}}^{\prime}: F_{P}\right] \cdot \operatorname{deg}(P) .
$$

This leads us to the following definition.

Definition 3.3.9 ([19] p.61) Let $P \in \mathcal{P}_{F}$ and let $P^{\prime} \in \mathcal{P}_{F^{\prime}}$ be a place such that $P^{\prime} \mid P$. The relative degree of $P^{\prime}$ over $P$ is $f\left(P \mid P^{\prime}\right)=\left[F_{P^{\prime}}^{\prime}: F_{P}\right]$. We will often just write $f$ when the places are obvious.

Example 3.3.10 Continuing from Example 3.3.8, the place $P_{p_{1}}$ of $K(x)$ lies under the places $P_{p_{1}, p_{2}}^{\prime}$ and $P_{p_{1},-p_{2}}^{\prime}$ of $K(x, y)$. In Example 3.1.22, for our proof that $P_{p_{1}, p_{2}}^{\prime}$ and $P_{p_{1},-p_{2}}^{\prime}$ are maximal ideals, we used the evaluation map to show that

$$
\mathcal{O}_{p_{1}, p_{2}} / P_{p_{1}, p_{2}}^{\prime} \cong K \cong \mathcal{O}_{p_{1},-p_{2}} / P_{p_{1},-p_{2}}^{\prime}
$$

Hence, $\operatorname{deg}\left(P_{p_{1}, p_{2}}^{\prime}\right)=1, \operatorname{deg}\left(P_{p_{1}, p_{2}}^{\prime}\right)=1$, and

$$
f\left(P_{p_{1}, p_{2}}^{\prime} \mid P_{p_{1}}\right)=\left[F_{P_{p_{1}, p_{2}}^{\prime}}: F_{P_{p_{1}}}\right]=[K: K]=1
$$

Similarly, $f\left(P_{p_{1}, p_{2}}^{\prime} \mid P_{p_{1}}\right)=1$. It is not always the case that $f=1$. In the next section 
we will look at some examples where this is indeed not the case.

\subsubsection{The Ramification Index of Places}

We now move to another comparison of $P^{\prime}$ and $P$.

Definition 3.3.11 ([19] p.61) Let $t, t^{\prime}$ be a local parameters of $P, P^{\prime}$ respectively, as in Defintion 3.1.15. Since $P \subseteq P^{\prime}$, and $t \in P$, by Theorem 3.1.13, there exist a unique positive integer $e$ and a unit $u \in \mathcal{O}_{P^{\prime}}$ such that $t=t^{\prime e} u$. We called $e$ the ramification index and denote it as $e\left(P^{\prime} \mid P\right)$ or simply as $e$ when the places are obvious. We say that $P^{\prime} \mid P$ is ramified if $e\left(P^{\prime} \mid P\right)>1$.

Recall that to each valuation we have defined an absolute value as in Definition 3.2.9. This definition leads us to some further properties of the ramification index.

Proposition 3.3.12 ([19] p.61) Let $F^{\prime} / F$ be function fields over $K$. Let $P$ be a place of $F$, lying under a place $P^{\prime}$ of $F^{\prime}$ with valuation rings $\mathcal{O}_{P}$ and $\mathcal{O}_{P^{\prime}}$, and discrete valuations $v_{P}$ and $v_{P^{\prime}}$ respectively. Let $F^{\times}$and $F^{\prime \times}$ denote the multiplicative groups of $F$ and $F^{\prime}$ respectively. Define $\left|F^{\times}\right|_{P}$ to be the subgroup of $\mathbb{R}^{\times}$given by the image of $\|_{P}$ and define $\left|F^{\prime \times}\right|_{P^{\prime}}$ similarly. The ramification index $e=e\left(P^{\prime} \mid P\right)$ satisfies

1. $v_{P^{\prime}}(z)=e \cdot v_{P}(z) \quad \forall z \in F$

2. $|z|_{P^{\prime}}=|z|_{P}^{e} \quad \forall z \in F$

3. $e=\left[\left|F^{\prime \times}\right|_{P^{\prime}}:\left|F^{\times}\right|_{P}\right]$

Proof. Let $t, t^{\prime}$ be a local parameters of $P, P^{\prime}$ respectively as in Definition 3.1.15. Since $P \subset P^{\prime}$, and $t \in P$, then as in Definition 3.3.11 there exists a unique integer $e$ and a unit $u \in \mathcal{O}_{P^{\prime}}$ such that $t=t^{\prime} u$. Now, for all $z \in F$, by Theorem 3.1.13, $z=t^{s} w$ for some unit $w \in \mathcal{O}_{P^{\prime}}$. So we have that

1. $v_{P^{\prime}}(z)=v_{P^{\prime}}\left(t^{s} w\right)=s \cdot v_{P^{\prime}}\left(\left(t^{\prime e} u\right)\right)+v_{P^{\prime}}(w)=s \cdot e+0=e \cdot v_{P}(z)$

2. $|z|_{P^{\prime}}=\left|t^{s} w\right|_{P^{\prime}}=\left|t^{s}\right|_{P^{\prime}}=|t|_{P^{\prime}}^{s}=\left|t^{\prime e} u\right|_{P^{\prime}}^{s}=\left|t^{\prime}\right|_{P^{\prime}}^{e s}=\left|t^{\prime s}\right|_{P^{\prime}}^{e}=|z|_{P}^{e}$ 
3. Observe that $|t|_{P}$ generates $\left|F^{\times}\right|_{P}$ and $\left|t^{\prime}\right|_{P^{\prime}}$ generates $\left|F^{\prime \times}\right|_{P^{\prime}}$. Since $t=t^{\prime e} u$, $|t|_{P} \in\left|F^{\prime \times}\right|_{P^{\prime}}$ and thus $\left|F^{\times}\right|_{P}$ is a subgroup $\left|F^{\prime \times}\right|_{P^{\prime}}$. Since $\mathbb{R}^{\times}$is abelian, we consider the quotient group $\frac{\left|F^{\prime \times}\right|_{P^{\prime}}}{\left|F^{\times}\right|_{P}}$. This group is generated by $\left|t^{\prime}\right|_{P^{\prime}} \cdot\left|F^{\times}\right|_{P}$. Since

$$
\left(\left|t^{\prime}\right|_{P^{\prime}} \cdot\left|F^{\times}\right|_{P}\right)^{e}=\left|t^{\prime}\right|_{P^{\prime}}^{e} \cdot\left|F^{\times}\right|_{P}=|t|_{P} \cdot\left|F^{\times}\right|_{P}=1 \cdot\left|F^{\times}\right|_{P}
$$

we have that $e=\left[\left|F^{\prime \times}\right|_{P^{\prime}}:\left|F^{\times}\right|_{P}\right]$.

We saw in Example 3.3.10 that both places lying over $P_{p_{1}}$ had the same relative degree $f$. This was no coincidence. Actually, their ramification index $e$ will be the same too. We will see later on why this is always the case for two distinct places of the quadratic $K(x, y)$ lying over a place of $K(x, y)$. This result will stem from the following lemma.

Lemma 3.3.13 ([19] p.89) Let $F^{\prime} / F$ be an algebraic extension on function fields. Let $P \in \mathcal{P}_{F}$ and $P^{\prime} \in \mathcal{P}_{F^{\prime}}$ be such that $P^{\prime} \mid P$. Let $\sigma$ be an automorphism of $F^{\prime}$ fixing $F$. Then $\sigma\left(P^{\prime}\right):=\left\{\sigma(z) \mid z \in P^{\prime}\right\}$ is a place of $F^{\prime}$. Moreover, we have

1. $v_{\sigma\left(P^{\prime}\right)}(w)=v_{P^{\prime}}\left(\sigma^{-1}(w)\right)$ for all $w \in F^{\prime}$.

2. $\sigma\left(P^{\prime}\right) \mid P$

3. $e\left(\sigma\left(P^{\prime}\right) \mid P\right)=e\left(P^{\prime} \mid P\right)$

4. $f\left(\sigma\left(P^{\prime}\right) \mid P\right)=f\left(P^{\prime} \mid P\right)$

Proof. We first prove that $\sigma\left(\mathcal{O}_{P^{\prime}}\right)$ is a valuation ring. Suppose $z \notin \sigma\left(\mathcal{O}_{P^{\prime}}\right)$ then $\sigma^{-1}(z) \notin \mathcal{O}_{P^{\prime}}$. Thus, $\left(\sigma^{-1}(z)\right)^{-1} \in \mathcal{O}_{P^{\prime}}$ and $\sigma^{-1}\left(z^{-1}\right) \in \mathcal{O}_{P^{\prime}}$. Hence, $z^{-1} \in \sigma\left(\mathcal{O}_{P^{\prime}}\right)$ and so $\sigma\left(\mathcal{O}_{P^{\prime}}\right)$ is a valuation ring. Clearly $\sigma\left(P^{\prime}\right)$ is its maximal ideal, and is thus a place of $F^{\prime}$ whose corresponding valuation ring is $\mathcal{O}_{\sigma\left(P^{\prime}\right)}=\sigma\left(\mathcal{O}_{P^{\prime}}\right)$. If $t^{\prime}$ is a local parameter of $P^{\prime}$ then $P^{\prime}=t^{\prime} \mathcal{O}_{P^{\prime}}$ by Definition 3.1.15. Now applying $\sigma$, we have that $\sigma\left(P^{\prime}\right)=\sigma\left(t^{\prime}\right) \cdot \sigma\left(\mathcal{O}_{P^{\prime}}\right)$. So $\sigma\left(t^{\prime}\right)$ is a local parameter of $\sigma\left(P^{\prime}\right)$. We now prove each of the numbered assertions of the Lemma. 
1. Let $w \in F^{\prime}$. Then since $\sigma$ is an automorphism, there exists $z \in F^{\prime}$ such that $\sigma(z)=w$. Writing $z=t^{\prime r} u$ where $u \in \mathcal{O}_{P^{\prime}}$ is a unit and $r=v_{P^{\prime}}(z)$, we have that $w=\sigma(z)=\sigma\left(t^{\prime}\right)^{r} \sigma(u)$, where $\sigma\left(t^{\prime}\right)$ is a local parameter of $\sigma\left(P^{\prime}\right)$ and $\sigma(u)$ is a unit of $\sigma\left(\mathcal{O}_{P^{\prime}}\right)$. Then by Theorem 3.2.3,

$$
v_{\sigma\left(P^{\prime}\right)}(w)=r=v_{P^{\prime}}(z)=v_{P^{\prime}}\left(\sigma^{-1}(\sigma(z))=v_{P^{\prime}}\left(\sigma^{-1}(w)\right) .\right.
$$

2. Since $\sigma$ fixes $F$ we have that $\sigma\left(P^{\prime}\right) \supseteq \sigma(P)=P$, and so $\sigma\left(P^{\prime}\right) \mid P$.

3. Let $t \in F$ be a local parameter of $P$ as in Definition 3.1.15. Then by Definition 3.3.11,

$$
e\left(\sigma\left(P^{\prime}\right) \mid P\right)=v_{\sigma\left(P^{\prime}\right)}(t)=v_{P^{\prime}}\left(\sigma^{-1}(t)\right)=v_{P^{\prime}}(t)=e\left(P^{\prime} \mid P\right) \cdot v_{P}(t)=e\left(P^{\prime} \mid P\right) .
$$

4. The automorphism $\sigma$ of $F^{\prime} / F$ induces an isomorphism $\bar{\sigma}: F_{P^{\prime}}^{\prime} \rightarrow F_{\sigma(P)}^{\prime}$ given by

$$
\bar{\sigma}\left(z+P^{\prime}\right)=\bar{\sigma}(z)+\bar{\sigma}\left(P^{\prime}\right)
$$

where $\bar{\sigma}$ is the identity on $F_{P}$. Hence $f\left(P^{\prime} \mid P\right)=f\left(\sigma\left(P^{\prime}\right) \mid P\right)$.

Example 3.3.14 Continuing from Example 3.3.8, the place $P_{p_{1}}$ of $K(x)$ lies under $P_{p_{1}, p_{2}}^{\prime}$ and $P_{p_{1},-p_{2}}^{\prime}$ of $K(x, y)$. We wish to compute the ramification indices $e\left(P_{p_{1}, p_{2}}^{\prime} \mid P_{p_{1}}\right)$ and $e\left(P_{p_{1},-p_{2}}^{\prime} \mid P_{p_{1}}\right)$. However, at this point we do not have the means to compute them. We may nevertheless use Lemma 3.3.13 to relate the ramification indices of these places. Consider the isomorphism $\tau: K(x, y) \rightarrow K(x, y)$ given by $\tau(x)=x$ and $\tau(y)=-y$. We notice that $\tau$ fixes $F$ and $\tau\left(P_{p_{1}, p_{2}}^{\prime}\right)=P_{p_{1},-p_{2}}^{\prime}$. So by Lemma 3.3.13,

$$
f\left(P_{p_{1}, p_{2}}^{\prime} \mid P_{p_{1}}\right)=f\left(P_{p_{1},-p_{2}}^{\prime} \mid P_{p_{1}}\right) \quad e\left(P_{p_{1}, p_{2}}^{\prime} \mid P_{p_{1}}\right)=e\left(P_{p_{1},-p_{2}}^{\prime} \mid P_{p_{1}}\right) .
$$

The equality of the relative degrees we have already seen in Example 3.3.10. We will verify later on that $e\left(P_{p_{1}, p_{2}}^{\prime} \mid P_{p_{1}}\right)=e\left(P_{p_{1}, p_{2}}^{\prime} \mid P_{p_{1}}\right)=1$ as well. 
Example 3.3.15 Let $K(x, y)$ be as in Lemma 3.3.2. Suppose that there exists an element $p_{1} \in K$ such that $\varphi\left(p_{1}\right)=0$. i.e $\left(x-p_{1}\right)$ is one of the distinct factors of $\varphi(x)$. Then $c\left(p_{1}, 0\right)=0$, so we may define the place $P_{p_{1}, 0}^{\prime}$ in $K(x, y)$ as before.

$$
\begin{aligned}
& \mathcal{O}_{p_{1}, 0}^{\prime}=\left\{\frac{a(x, y)}{b(x, y)} \in K(x, y) \mid b\left(p_{1}, 0\right) \neq 0\right\} \\
& P_{p_{1}, 0}^{\prime}=\left\{\frac{a(x, y)}{b(x, y)} \in K(x, y) \mid a\left(p_{1}, 0\right)=0, b\left(p_{1}, 0\right) \neq 0\right\}
\end{aligned}
$$

We know that $y^{2}=\varphi(x)=\left(x-p_{1}\right) g(x)$ where $g(x) \in K[x]$ and $g\left(p_{1}\right) \neq 0$. Taking the valuation of both sides we have that

$$
\begin{aligned}
v_{P_{p_{1}, 0}^{\prime}}\left(y^{2}\right) & =v_{P_{p_{1}, 0}^{\prime}}\left(\left(x-p_{1}\right) g(x)\right), \\
2 v_{P_{p_{1}, 0}^{\prime}}^{\prime}(y) & =v_{P_{p_{1}, 0}^{\prime}}\left(x-p_{1}\right)+v_{P_{p_{1}, 0}^{\prime}}(g(x)), \\
2 v_{P_{p_{1}, 0}^{\prime}}^{\prime}(y) & =e \cdot v_{P_{p_{1}}}\left(x-p_{1}\right)+0 \\
2 v_{P_{p_{1}, 0}^{\prime}}(y) & =e \cdot 1 .
\end{aligned}
$$

Therefore $e$ is a nonzero multiple of 2 and hence $P_{p_{1}, 0}^{\prime} \mid P_{p_{1}}$ is ramified. We will see later on that in fact $e=2$.

Combining our results on the ramification index we have the following proposition.

Proposition 3.3.16 Let $F^{\prime} / F$ be an algebraic extension of function fields. Let $P^{\prime} \in$ $\mathcal{P}_{F^{\prime}}$ and $P \in \mathcal{P}_{F}$. Then the following are equivalent:

1. $P^{\prime} \mid P$

2. $v_{P^{\prime}}(z)=e \cdot v_{P}(z) \quad \forall z \in F$

3. $|z|_{P^{\prime}}=|z|_{P}^{e} \quad \forall z \in F$. In particular $\|_{P^{\prime}}$ and $\|_{P}$ are equivalent absolute values on $F$ and $\|_{P^{\prime}}^{1 / e}$ extends $\|_{P}$.

Proof. $(1) \Rightarrow(2),(1) \Rightarrow(3)$ : are restatements of Proposition 3.3.12

$(2) \Rightarrow(1)$ : Let $z \in P$. Then $e \cdot v_{P}(z)>0$ by Remark 3.1 .12 , an so by (2) we have 
that $v_{P^{\prime}}(z)>0$. Thus, $z \in P^{\prime}$. This proves that $P \subseteq P^{\prime}$.

$(3) \Rightarrow(1)$ : Let $z \in P$ then $|z|_{P}<1$ by Definition 3.2.9, and by the equivalence of $\|_{P^{\prime}}$ and $\|_{P},|z|_{P^{\prime}}<1$. This implies that $z \in P^{\prime}$. This proves that $P \subseteq P^{\prime}$.

\subsubsection{Basic Galois Theory}

We move on to an important property of quadratic extensions; namely that they are Galois extensions.

Definition 3.3.17 For any finite extension $F^{\prime} / F$, let $A u t_{F^{\prime} / F}$ be the group of automorphisms of $F^{\prime}$ that fix $F$. If $\left|A u t_{F^{\prime} / F}\right|=\left[F^{\prime}: F\right]$ then the extension is called Galois. For any Galois extension we may define the Galois norm (or just norm) $N_{F^{\prime} / F}: F^{\prime} \rightarrow F$ by

$$
N_{F^{\prime} / F}(z)=\prod_{\sigma \in A u t_{F^{\prime} / F}} \sigma(z) \text { for all } z \in F^{\prime}
$$

The norm indeed maps to $F$. We will only prove this for the quadratic case. For a general proof, and more information Galois extensions, see [2].

Proposition 3.3.18 Let $F^{\prime}=K(x, y)$ where $K(x, y)$ is a quadratic extendion of $K(x)$ as in Lemma 3.3.2. Then there are exactly two automorphisms of $F^{\prime}$ that restrict to the identity on $F$; namely the identity map, $I_{F^{\prime}}$, and $\tau$ where $\tau(y)=-y$.

Proof. Let $\sigma$ be an automorphism of $F^{\prime}$ that restricts to the identity on $F$. Then,

$$
(\sigma(y))^{2}=y^{2}
$$

Therefore the only possibilities are $\sigma(y)=y$ and $\sigma(y)=-y$.

These elements $I_{F^{\prime}}$ and $\tau$ clearly form a group, called the Galois group of $F^{\prime} / F$. We will call the identity map the trivial Galois automorphism, and the other map the non-trivial Galois automorphism. 
Let $F^{\prime}=K(x, y)$ be a quadratic extension of $F=K(x)$ as in Lemma 3.3.2. Let $\tau$ be the non-trivial Galois automorphism. Then the norm map $N_{F^{\prime} / F}: F^{\prime} \rightarrow F$ is defined by

$$
N(z)=z \cdot \tau(z) \text { for all } z \in F^{\prime} .
$$

Proposition 3.3.19 Let $F^{\prime}=K(x, y)$ be a quadratic extension of $F=K(x)$ as in Lemma 33.2. Then the Galois norm has the property that $N_{F^{\prime} / F}(z) \in F$ for all $z \in F^{\prime \prime}$.

Proof. For all $z \in K(x, y)$ we have that $z=\frac{a(x)+b(x) y}{c(x)}$ for some $a(x), b(x), c(x) \in K[x]$ with $c(x) \neq 0$. Then

$$
N_{F^{\prime} / F}(z)=\left(\frac{a(x)+b(x) y}{c(x)}\right)\left(\frac{a(x)-b(x) y}{c(x)}\right)=\frac{a(x)^{2}-b(x)^{2} y^{2}}{c(x)^{2}} \in K(x) .
$$

\subsection{Completions of Algebraic Extensions}

Our goal in the next few sections is to finally be able to describe the discrete valuations, ramification indices, relative degrees, and absolute values of all the places of the quadratic extension $K(x, y) / K(x)$.

We begin by proving a preliminary result that will aid in calculating how many places lie over a given place $P$ in $K(x)$.

Corollary 3.4.1 Let $F^{\prime} / F$ be a finite algebraic extension of function fields. Let $P \in \mathcal{P}_{F}$. The number of distinct $P^{\prime} \in \mathcal{P}_{F^{\prime}}$ such that $P^{\prime} \mid P$ is equal to the number of distinct equivalence classes of absolute values extending $\|_{P}$.

Proof. By Proposition 3.3.16, $P^{\prime} \mid P$ if and only if $\|_{P^{\prime}}$ extends $\|_{P}$. From Proposition 3.2 .12 , we know that there is a one-to-one correspondence between the places of $F^{\prime}$ and the distinct equivalence classes of absolute values on $F^{\prime}$. Thus the number of $P^{\prime} \mid P$ is cqual to the number of distinct absolute values of $F^{\prime}$ extending $\|_{P}$. 
We will use Corollary 3.4.1 to calculate the number of places over a given place by calculating the possible absolute value extending $\|_{P}$. This is the goal of this section.

Let $F^{\prime}$ be an extension of a function field $F$. From Proposition 3.3.12 (2), for all places $P^{\prime} \mid P$ we see that $\|_{P}$ and $\|_{P^{\prime}}$ are equivalent absolute values. For this reason, a sequence that is Cauchy in $F$ is also Cauchy in $F^{\prime}$ (Definition 3.2.14). This gives a natural embedding of the completions $\hat{F} \hookrightarrow \hat{F}^{\prime}$ (Definition 3.2.19). This in turn allows us to consider $\hat{F}$ as a subfield of $\hat{F}^{\prime}$.

Theorem 3.4.2 ([7] p.569) Let $\|$ be an absolute value of $\hat{F}$ such that $\hat{F}$ is complete relative to $\|$. Let $E$ be a finite Galois extension of $\hat{F}$. If $\|$ can be extended to an absolute value on $E$ then this may be done in only one way and the extension of $\|$ on $E$ is given by

$$
|a|=\left|N_{E / \hat{F}}(a)\right|^{1 /[E: \hat{F}]} .
$$

To prove this theorem we need the following lemma.

Lemma 3.4.3 Let $\|$ be an absolute value of $\hat{F}$ such that $\hat{F}$ is complete relative to \|. Let $E$ be a finite Galois extension of $\hat{F}$. Let $u_{1}, \ldots, u_{r}$ be linearly independent elements of $E$ over $F$. Let $\left\{a_{n}\right\}$ be a sequence of elements of $E$ of the form $a_{n}=\sum_{i=1}^{r} a_{n i} u_{i}$ where $a_{n i} \in F$. Then $\left\{a_{n}\right\}$ is a Cauchy sequence in $E$ if and only if the sequences $\left\{a_{n i}\right\}$, for all $1 \leq i \leq r$, are Cauchy sequences in $F$. Moreover, $E$ is complete.

Proof. One direction is clear, namely if every $\left\{a_{n i}\right\}$ is Cauchy then so is $\left\{a_{n}\right\}$. Conversely, suppose that $\left\{a_{n}\right\}$ is Cauchy. We will prove by induction on $r$ that $\left\{a_{n i}\right\}$ is Cauchy for all $1 \leq i \leq r$.

If $r=1$ then clearly $\left\{a_{n 1}\right\}_{n>0}$ is Cauchy. We now use the induction hypothesis that if a sequence

$$
\left\{d_{n}\right\}_{n>0}=\left\{\sum_{i=1}^{r-1} d_{n i} u_{i}\right\}_{n>0}
$$

is Cauchy, then so are $\left\{d_{n i}\right\}_{n>0}$ for $i=1, \ldots, r-1$. Suppose that $\left\{a_{n r}\right\}_{n>0}$ is not a Cauchy sequence. Our goal is to contradict the linear independence of $u_{1}, \ldots, u_{n}$. We 
will do this by creating a series of sequences whose limit points will give the desired contradiction.

It follows from the definition of a Cauchy sequence (Defintion 3.2.14) that there exists a real number $\epsilon>0$ such that for any positive integer $N$ there exist integers $p, q \geq N$ such that

$$
\left|a_{p r}-a_{q r}\right|>\epsilon
$$

Since this is true for any $N$, we may generate a sequence of $p^{\prime} s$ and $q^{\prime} s$ in the following manner. Pick an $N$ and get a pair $\left(p_{1}, q_{1}\right)$ which satisfy Equation (3.19). Now we may pick another $N>p_{1}, N>q_{1}$, to get another pair $\left(p_{2}, q_{2}\right)$, and so on. Thus we may create a sequence of pairs of positive integers $\left(p_{k}, q_{k}\right)$ where $p_{1}<p_{2}<\ldots$ and $q_{1}<q_{2}<\ldots$ such that $\left|a_{p_{k} r}-a_{q_{k} r}\right|>\epsilon$. Since $a_{n r} \in F$ for all $n$, we have that $\left(a_{p_{k} r}-a_{q_{k} r}\right)^{-1}$ exists in $F$ for all $k>0$. With this we may form a new sequence $\left\{b_{k}\right\}_{k>0}$ where

$$
b_{k}=\left(a_{p_{k} r}-a_{q_{k} r}\right)^{-1}\left(a_{p_{k}}-a_{q_{k}}\right) .
$$

Since $\left|a_{p_{k} r}-a_{q_{k} r}\right|^{-1}<\epsilon^{-1}$ and $\left(a_{p_{k}}-a_{q_{k}}\right) \rightarrow 0$, we have that $b_{k} \rightarrow 0$. On the other hand,

$b_{k}=\left(a_{p_{k} r}-a_{q_{k} r}\right)^{-1}\left(a_{p_{k}}-a_{q_{k}}\right)=\left(a_{p_{k} r}-a_{q_{k} r}\right)^{-1}\left(\sum_{i=1}^{r}\left(a_{p_{k} i}-a_{q_{k}}\right) u_{i}\right)=\sum_{j=1}^{r-1} \beta_{k j} u_{j}+u_{r}$

where $\beta_{k j}=\left(a_{p_{k} r}-a_{q_{k} r}\right)^{-1}\left(a_{p_{k} j}-a_{q_{k} j}\right)$. Let

$$
c_{k}:=\sum_{j=1}^{r-1} \beta_{k j} u_{j} .
$$

Then $c_{k}$ converges to $-u_{r}$ and so $c_{k}$ is Cauchy. Hence, by the induction hypothesis every $\left\{\beta_{k j}\right\}_{k>0}$ is Cauchy for $j=1, \ldots, r-1$. Since $F$ is complete relative to $\|$, $\left\{\beta_{k j}\right\}_{k>0}$ converges to $\beta_{j}$ for some $\beta_{j} \in F$. Then taking limits in Equation (3.20) we 
obtain

$$
0=\sum_{j=1}^{r-1} \beta_{j} u_{j}+u_{r}
$$

This contradicts the linear independence of $u_{1}, \ldots, u_{r}$. Thus our assumption that $\left\{a_{n r}\right\}_{n>0}$ is not Cauchy is false.

So we have proven that if $\left\{a_{n}\right\}$ is Cauchy then $\left\{a_{n r}\right\}_{n>0}$ is Cauchy. Now, Given that $\left\{a_{n r}\right\}$ is Cauchy, define $d_{n}=a_{n}-a_{n r} u_{r}=\sum_{i=1}^{r-1} a_{n i} u_{i}$. Since $\left\{d_{n}\right\}$ is the difference of two Cauchy sequences, it is Cauchy and thus by the induction hypothesis all the $\left\{a_{n i}\right\}$ are Cauchy for $i=1, \ldots, r$.

It is clear that if $\left\{a_{n}\right\} \rightarrow 0$ then $\left\{a_{n i}\right\} \rightarrow 0$ for all $i=1, \ldots, r$. Consequently, if we take $u_{1}, . ., u_{n}$ to be a basis of $E / \hat{F}$ then $E$ is complete.

While Theorem 3.4.2 is true as stated, the proof requires showing that the norm map is continuous. As such, we will only prove the case where $[E: \hat{F}]=2$.

Proof. (of Theorem 3.4.2 for $[E: \hat{F}]=2$ )

Let $2=[E: \hat{F}]$ and let $\{1, y\}$ be a basis of $E / \hat{F}$. If Equation (3.18) does not hold, then there exists an $a \in E$ where, $a=a_{1}+a_{2} y$, such that $|a|^{2} \neq\left|N_{E / \hat{F}}(a)\right|$. By replacing $a$ with $a^{-1}$ if necessary, we may assume that $\left|a^{2}\right|<\left|N_{E / \hat{F}}(a)\right|$. Set $b:=a^{2} N_{E / F}(a)^{-1}$. Then $|b|<1$ and $N_{E / \hat{F}}(b)=1$. Since $|b|<1, \lim _{k \rightarrow \infty} b^{k}=0$. Hence, if we write $b^{k}=\beta_{1, k}+\beta_{2, k} y$, then $\lim _{k \rightarrow \infty} \beta_{i, k}=0$ for both $i=1,2$ (Lemma 3.4.3), and by Lemma $3.4 .3, \lim _{k \rightarrow \infty}\left(\beta_{1, k}-\beta_{2, k} y\right)=0$ as well. Since

$$
\begin{aligned}
\left|N_{E / \hat{F}}\left(b^{k}\right)\right| & =\left|\left(\beta_{1, k}-\beta_{2, k} y\right)\left(\beta_{1, k}-\beta_{2, k} y\right)\right| \\
& =\left|\beta_{1, k}-\beta_{2, k} y\right|\left|\beta_{1, k}-\beta_{2, k} y\right| \\
& \rightarrow 0 \text { as } k \rightarrow \infty
\end{aligned}
$$

we have that $\lim _{k \rightarrow \infty} N_{E / F}\left(b^{k}\right)=0$. Therefore, $1=\lim _{k \rightarrow \infty} N_{E / F}\left(b^{k}\right)=0$. This contradiction proves (3.18).

Notice that in Theorem 3.4.2, we had not actually shown that I| may be extended 
to $E$. This will also be the case for next few results. Once we know more about the possibilities for absolute values extending $\|$ on $K(x)$ it will be easier to prove their existence.

Corollary 3.4.4 Let $K(x, y)$ be a quadratic extension of $K(x)$ as in Lemma 3.3.2. Let $\|$ be an absolute value on $K(x)$. If $\|$ may be extended to an absolute value on $K(x, y)$ then

$$
\widehat{K(x)}(y) \cong \widehat{K(x, y)}
$$

Proof. Notice that $\widehat{K(x)}(y)$ is a field extension of $\widehat{K(x)}$, which may be viewed as a vector space over $\widehat{K(x)}$. Define $\Phi$ from the vector space of sequences in $K(x)$ over $y$ to the sequences of $K(x, y)$ given by $\left.\Phi\left(\left\{a_{i}\right\}+\left\{b_{i}\right\} y\right)=\left\{a_{i}+b_{i} y\right\}\right)$. By Lemma 3.4.3, $\left\{a_{i}+b_{i} y\right\}$ is Cauchy if and only if $\left\{a_{i}\right\}$ and $\left\{b_{i}\right\}$ are Cauchy in $F$. Then clearly $\Phi$ is a ring isomorphism. Thus $\widehat{K(x)}(y) \cong \widehat{K(x, y)}$ as desired.

Proposition 3.4.5 Let $K(x, y)$ be a quadratic extension of $K(x)$ as in Lemma 3.3.2. Let $\|$ be a non-trivial absolute value of $K(x)$ and let $\widehat{K(x)}$ denote the completion of $K(x)$ relative to $\|$ as in Definition 3.2.19. Suppose that $\|$ may be extended to an absolute value on $K(x, y)$ and let $\widehat{K(x, y)}$ denote the completion of $K(x, y)$ relative to this extended absolute value. Let $\sigma: K(x) \hookrightarrow \widehat{K(x)}$ be the natural embedding. Then there exists $\sigma^{\prime}: K(x, y) \hookrightarrow \widehat{K(x, y)}$ which is an embedding extending $\sigma$, i.e we have the following diagram.

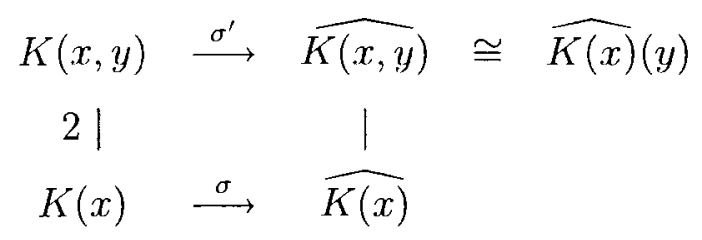

In this situation there are exactly two distinct possibilities for $\sigma^{\prime}$.

Proof. Suppose that $\sigma^{\prime}: K(x, y) \rightarrow \widehat{K(x, y)}$ is a homomorphism such that $\left.\sigma^{\prime}\right|_{K(x)}=$ $\sigma$. Let $m_{y, F}(X)=X^{2}-\varphi(x)$ denote the minimum polynomial of $y$ over $F$. Then 
$m_{y, F}(y)=0$ and taking $\sigma^{\prime}$ of both sides we see that.

$$
\begin{aligned}
\sigma^{\prime}\left(m_{y, F}(y)\right) & =\sigma^{\prime}(0), \\
\sigma^{\prime}\left(y^{2}-\varphi(x)\right) & =0 \\
\sigma^{\prime}(y)^{2} & =\sigma^{\prime}(\varphi(x)), \\
\sigma^{\prime}(y)^{2} & =\varphi(x), \\
\sigma^{\prime}(y)^{2} & =y^{2} .
\end{aligned}
$$

Thus there are only two possible choices for $\sigma^{\prime}$,

$$
\sigma^{\prime}(a+b y)=\sigma(a)+\sigma(b) y \quad \text { or } \quad \sigma^{\prime}(a+b y)=\sigma(a)-\sigma(b) y
$$

for $a, b \in K(x)$, each of which is easily checked to be an embedding extending $\sigma$.

We may now describe the possible absolute values of a quadratic extension extending an absolute value of $K(x)$. Before we do so, we give the following definition.

Definition 3.4.6 Let $L$ be a field extension of $K$. Let $\sigma: K[X] \rightarrow L[X]$ be a homomorphism. Let $a(X) \in K[X]$ be an monic irreducible polynomial of degree $d$. We say that the polynomial $a(X)$ splits in $L[X]$ if there exist elements $a_{1}, \ldots, a_{d} \in L$ such that $\sigma(a(X))=\left(X-a_{1}\right) \cdots\left(X-a_{d}\right) \in L[X]$.

Theorem 3.4.7 Let $F^{\prime}=K(x, y)$ be a quadratic extension of $F=K(x)$ as in Lemma 3.3.2. Let $\|_{F}$ be a nontrivial absolute value on $F$. Let $\sigma: K(x) \rightarrow \widehat{K(x)}$ be the natural embedding. Let $m_{y, F}(X)$ be the minimal polynomial of $y$ over $F$. Let $z=\alpha+\beta y \in F^{\prime}$ for some $\alpha, \beta \in K(x)$. Then either

1. $\sigma\left(m_{y, K(x)}(X)\right)$ does not split in $\widehat{K(x)}$. Then there is a unique absolute value $\|_{F^{\prime}}$ (up to equivalence) extending $\|_{F}$ given by

$$
|z|_{F^{\prime}}=\left|N_{F^{\prime} / F}(z)\right|_{F}^{1 / 2}
$$


2. $\sigma\left(m_{y, K(x)}(X)\right)$ splits in $\widehat{K(x)}$. Let $\theta \in \widehat{K(x)}$ be a root of $\sigma\left(m_{y, K(x)}(X)\right)$. Then there are two unique absolute values (up to equivalence) extending $\|_{F}$ given by

$$
|\alpha+\beta y|_{F^{\prime}}=|\alpha+\beta(-\theta)|_{F} \quad \text { and } \quad|\alpha+\beta y|_{F^{\prime}}=|\alpha+\beta \theta|_{F}
$$

This exhausts all possible cases.

Proof. 1. Suppose that $\sigma\left(m_{y, K(x)}(X)\right)$ does not split in $\widehat{K(x)}$. Then $[\widehat{K(x, y)}$ : $\widehat{K(x)}]=2$. By Theorem 3.4.2, if there is an absolute value $\|_{F^{\prime}}$ extending $\|_{F}$ then it is given by

$$
|z|_{F^{\prime}}=\left|N_{F^{\prime} / F}(z)\right|_{F}^{1 / 2}
$$

Thus we need to show that this is indeed an absolute value on $F^{\prime}$ extending $\|_{F}$.

(a) If $z \in F$ then $\left|N_{F^{\prime} / F}(z)\right|_{F}^{1 / 2}=\left|z^{2}\right|_{F}^{1 / 2}=|z|_{F}$

(b) $0=\left|N_{F^{\prime} / F}(z)\right|_{F}^{1 / 2} \Leftrightarrow N_{F^{\prime} / F}(z)=0 \Leftrightarrow z=0$

(c) $|v z|_{F^{\prime}}=\left|N_{F^{\prime} / F}(v z)\right|_{F}^{1 / 2}=\left|N_{F^{\prime} / F}(v) \cdot N_{F^{\prime} / F}(z)\right|_{F}^{1 / 2}=|v|_{F^{\prime}}|z|_{F^{\prime}}$ for all $v, z \in$ $F^{\prime}$

(d) Let $\tau$ be the nontrivial Galois automorphism. Then for all $z, v \in F^{\prime}$

$$
\begin{aligned}
\left|N_{F^{\prime} / F}(v+z)\right|_{F}^{1 / 2} & =|(z+v) \tau(z+v)|_{F}^{1 / 2} \\
& =|z \tau(z)+v \tau(v)+v \tau(z)+z \tau(v)|_{F}^{1 / 2} \\
& \leq\left(|z \tau(z)+v \tau(v)|_{F}+|z \tau(v)+v \tau(z)|_{F}\right)^{1 / 2} \\
& \leq\left(|z \tau(z)+v \tau(v)|_{F}\right)^{1 / 2}+\left(|z \tau(v)+v \tau(z)|_{F}\right)^{1 / 2} \\
& \leq\left(|z \tau(z)+v \tau(v)|_{F}\right)^{1 / 2} \\
& \leq(|z \tau(z)|)^{1 / 2}+\left(|v \tau(v)|_{F}\right)^{1 / 2} \\
& =\left|N_{F^{\prime} / F}(z)\right|_{F^{\prime}}^{1 / 2}+\left|N_{F^{\prime} / F}(v)\right|_{F^{\prime}}^{1 / 2}
\end{aligned}
$$

Thus Equation( 3.22) defines an absolute value $\|_{F^{\prime}}$ on $F^{\prime}$ extending $\|_{F}$ and by Theorem 3.4 .2 it is unique. 
2. Suppose that $\sigma\left(m_{y, K(x)}(X)\right)$ splits in $\hat{F}=\widehat{K(x)}$. Then $[\widehat{K(x, y)}: \widehat{K(x)}]=1$ and $\widehat{K(x, y)}=\widehat{K(x)}$. Let $\theta \in \widehat{K(x)}$ be a root of $\sigma\left(m_{y, K(x)}(X)\right)$. Since $\theta \in \widehat{K(x)}$ there exist a Cauchy sequence $\left\{a_{i}\right\} \in F$ converging to $\theta$ with respect to $\|_{F}$. Let $\|_{F^{\prime}}$ be an absolute value of $F^{\prime}$ extending $\|_{F}$. Since $\|_{F^{\prime}}$ extends $\|_{F},\left\{a_{i}\right\}$ converges with respect to $\|_{F^{\prime}}$. Furthermore, $\left\{m_{y, K(x)}\left(a_{i}\right)\right\} \rightarrow 0$ with respect to $\|_{F^{\prime}}$ and thus $\left\{a_{i}\right\} \rightarrow \pm y$. Suppose that $\left\{a_{i}\right\}$ converges to $y$. Then for all $\alpha, \beta \in F$

$$
\alpha+\beta y=\lim _{i \rightarrow \infty} \alpha+\beta a_{i}=\alpha+\beta \theta
$$

Similarly, if $\left\{a_{i}\right\}$ converges to $-y$ then $\alpha+\beta y=\alpha+\beta(-\theta)$. Since the sequence $\left\{a_{i}\right\}$ converges to either $\pm y$ either

$$
|\alpha+\beta y|_{F^{\prime}}=|\alpha+\beta(-\theta)|_{F} \quad \text { or } \quad|\alpha+\beta y|_{F^{\prime}}=|\alpha+\beta \theta|_{F} .
$$

These correspond directly to the two choices of embeddings $\sigma^{\prime}$ extending $\sigma$ of Proposition 3.4.5. The two absolute values defined in (3.23) are indeed absolute values as they inherit the properties directly from the absolute value defined on $\hat{F}$. To prove that the two absolute values are distinct, we do a proof by contradiction. If the two absolute values are the same then they correspond to the same valuation ring $\mathcal{O}^{\prime} \subset F^{\prime}$. In particular, if $\alpha+\beta y \in \mathcal{O}^{\prime}$ then $\alpha-\beta y \in \mathcal{O}^{\prime}$. Since the minimal polynomial $\sigma\left(m_{y, K(x)}(X)\right)$ splits,

$$
0=\sigma\left(m_{y, K(x)}\right)(y)=(y+\theta)(y-\theta) \text { for some } \theta \in \hat{F} .
$$

Without loss of generality we assume that $y-\theta=0$. Since $F$ is dense in $\hat{F}$, there exists a sequence $\left\{a_{i}\right\} \in F$ such that $\lim _{i \rightarrow \infty} a_{i}=\theta$. By Corollary 3.4.4, this implies that $\left|a_{i}-y\right| \rightarrow 0$. Let $t \in F^{\prime}$ be a local parameter of the place corresponding to $\|_{F}^{\prime}$ as in Proposition 3.2.12. Then we choose $n \in \mathbb{Z}$ such that 
$\left|t^{n}\right|_{F^{\prime}}<|2 y|_{F^{\prime}}$. Now choose an $i>0$ such that $\left|a_{i}-y\right|_{F^{\prime}}<\left|t^{n}\right|_{F^{\prime}}$. Then

$$
1>\frac{\left|a_{i}-y\right|_{F^{\prime}}}{\left|t^{n}\right|_{F^{\prime}}} \text { and thus } \frac{a_{i}-y}{t^{n}} \in \mathcal{O}^{\prime} .
$$

Subsequently, by the equivalence of the absolute values we have that

$$
1>\frac{\left|a_{i}+y\right|_{F^{\prime}}}{\left|t^{n}\right|_{F^{\prime}}} \text { and thus } \frac{a_{i}+y}{t^{n}} \in \mathcal{O}^{\prime} .
$$

However subtracting these two elements we see that

$$
\left|\frac{a_{i}-y}{t^{n}}-\frac{a_{i}+y}{t^{n}}\right|_{F^{\prime}}=\left|\frac{2 y}{t^{n}}\right|_{F^{\prime}}>1 .
$$

Thus $\mathcal{O}^{\prime}$ is not closed and hence not a ring. So the two absolute values are indeed distinct (up to equivalence). This proves that in this case there are exactly two absolute values extending any $\|_{F}$ (up to equivalence).

\subsection{The Places of a Quadratic Extension of $K(x)$}

In keeping with the geometric analogy mentioned in the introduction, we use Theorem 3.4.7 to explicitly describe all the places of quadratic extension. We partition the places of $K(x)$ into four separate categories. To each category we dedicate a subsection, in which we compute all the possible places of $K(x, y)$ lying over the particular type of places of $K(x)$. As a result of Theorem 3.4.7, our process will provide a complete list of the places of $K(x, y)$. This is further justified using the following lemma.

Lemma 3.5.1 Let $F^{\prime}=K(x, y)$ be a quadratic extension of $F=K(x)$ as in Lemma 3.3.2 and let $P \in \mathcal{P}_{F}$. Then there exists at least one but at most two places $P^{\prime} \in \mathcal{P}_{F^{\prime}}$ such that $P^{\prime} \mid P$.

Proof. Let $\|_{P}$ be the absolute value of $F$ corresponding to a place $P$. From Theorem 
3.4.7 we know that there is at least one and at most two absolute values of $K(x, y)$ extending $\|_{P}$. By Corollary 3.4.1, the number of places lying over $P$ is equal to the number of equivalence classes of absolute values extending the absolute value $\|_{P}$. Hence there is at least one and at most two places in $\mathcal{P}_{F^{\prime}}$ lying over $P$.

We may finally describe a places of the quadratic extension $K(x)$.

Let $F^{\prime}=K(x, y)$ be a quadratic extension of $F=K(x)$ as in Lemma 3.3.2. Let $m_{y, F}(X)=X^{2}-\varphi(x)$. Note that $m_{y, F}(X)$ is an irreducible polynomial and thus the minimal polynomial of $y$. Let $P$ be a place of $K(x)$. By Theorem 3.4.7 we know all absolute values extending $\|_{P}$. By Corollary 3.4.1, we know that the corresponding places of the absolute values extending $\|_{P}$ are all the places lying over $P$. By Corollary 3.3.7, we know that every place of $K(x, y)$ lies over a unique place $P$ in $K(x)$. From Theorem 3.1.20, we know all the places of $K(x)$. Thus this process will give us all the places of $K(x, y)$.

We begin by investigating the conditions under which $m_{y, F}(X)=X^{2}-\varphi(x)$ splits over $\widehat{K(x)}$. Since $m_{y, F}(X)$ is a quadratic polynomial in $X, m_{y, F}(X)$ splits if and only if there exists an element $a$ in $\widehat{K(x)}$ such that $(y-a)(y+a)=y^{2}-\varphi(x)$ i.e. if and only if there exists an $a \in \widehat{K(x)}$ such that

$$
\begin{aligned}
(y-a)(y+a) & =y^{2}-\varphi(x) \\
y^{2}-a^{2} & =y^{2}-\varphi(x) \\
a^{2} & =\varphi(x)
\end{aligned}
$$

Suppose that such an element $a$ exists. Let $t$ be a local parameter at $P$ as in Defintion 3.1.15. We now compute a Laurent series expansion for $a$ with respect to $t$ over $\mathcal{O}_{P}$ (Proposition 3.2.21). Since $\varphi(x) \in K(x) \subset \widehat{K(x)}$, by Corollary 3.2 .23 we may represent it uniquely in the form $\varphi(x)=\sum_{i=m}^{\infty} \varphi_{i}(x) t^{i}$ for some $\varphi_{i}(x) \in \mathcal{O}_{P}^{*}$ where $\varphi_{m}(x) \neq 0$. Then we may represent $a$ uniquely as $a=\sum_{i=k}^{\infty} a_{i}(x) t^{i}$ for some $a_{i}(x) \in$ 
$\mathcal{O}_{P}$ and we have that

$$
\begin{aligned}
\varphi(x) & =a^{2} \\
\sum_{i=m}^{\infty} \varphi_{i}(x) t^{i} & =\left(\sum_{i=k}^{\infty} a_{i}(x) t^{i}\right)^{2} \\
\sum_{i=m}^{\infty} \varphi_{i}(x) t^{i} & =\sum_{i=2 k}^{\infty}\left(\sum_{j=k}^{i} a_{j}(x) a_{i-j}(x)\right) t^{i} .
\end{aligned}
$$

Equating coefficients, the first thing we notice is that $m=2 k$. Consequently, equating coefficients thereafter, we have that

$$
\begin{aligned}
a_{m}(x)^{2} & =\varphi_{m}(x) \\
2 a_{m}(x) a_{m+1}(x) & =\varphi_{m+1}(x)
\end{aligned}
$$

If $\varphi_{m}(x)$ is a square in $K(x)$ these equations may be solved recursively to find the coefficients of $a$. Alternatively, if $\varphi_{m}(x)$ is not a square in $K(x)$ then there is no element $a \in \widehat{K(x)}$ to satisfy Equation (3.25). Consequently, $m_{y, F}(X)$ splits in $\widehat{K(x)}$ if and only if $\varphi_{m}(x)$ is a square in $K(x)$ and by Equation (3.24) that $v_{P}(\varphi(x))=2 k$ for some $k \in \mathbb{Z}$. These are necessary and sufficient conditions for $m_{y, F}(X)$ to split in $\widehat{K(x)}$.

We now examine all possible places $P$ of $K(x)$. Using the notation above, we partition the places of $K(x)$ into four cases.

Case 1. $P=P_{p(x)}$ where $p(x) \mid \varphi(x)$ :

Since $\varphi(x)$ is as in Lemma 3.3.2, it is the product of distinct irreducible polynomials. Therefore, $\varphi(x)=p(x) g(x)$ for some $g(x) \in K[x]$ where $p(x) \nmid g(x)$. By Corollary 3.2.6, $v_{P}(\varphi(x))=1$, which is of course odd. Thus, by the above, $m_{y, F}(X)$ does not split in $\widehat{K(x)}$. Consequently, by Theorem 3.4 .7 , we know that there is a unique 
absolute value $\|\left.\right|_{P^{\prime}}$ extending $\|_{P}$ given by $|z|_{P^{\prime}}=\left|N_{F^{\prime}} / F(z)\right|_{P}^{1 / 2}$ for all $z \in K(x, y)$. Therefore there is a unique place $P^{\prime} \mid P$, given by

$$
P^{\prime}=\left\{\left.z \in K(x, y)|| N_{F^{\prime} / F}(z)\right|^{1 / 2}<1\right\}
$$

Case 2. $P=P_{p(x)}$ where $p(x) \nmid \varphi(x)$ :

Since $p(x) \nmid \varphi(x)$ we know that $v_{P}(\varphi(x))=0$. So in the above notation $m=0$. Now we need to check if $\varphi_{0}(x)$ is a square in $K(x)$. We know from Example 3.1.16 that $p(x)$ is a local parameter at $P$, so by Proposition 3.2.21, we may uniquely express $\varphi(x)$ as

$$
\begin{aligned}
\varphi(x) & =\sum_{i=0}^{k} \varphi_{i}(x) p(x)^{i} \\
& =p(x)\left(\sum_{i=1}^{k} \varphi_{i}(x) p(x)^{i-1}\right)+\varphi_{0}(x)
\end{aligned}
$$

for some $k>0$ where $\operatorname{deg}\left(\varphi_{i}(x)\right)<\operatorname{deg}(p(x))$ for all $0 \leq i \leq k$. From the manner in which we have written this equation, if $\operatorname{deg}(\varphi(x)) \geq \operatorname{deg}(p(x))$ it is plain to see that $\varphi_{0}(x)$ is the remainder of the division algorithm when dividing $\varphi(x)$ by $p(x)$. If $\operatorname{deg}(\varphi(x))<\operatorname{deg}(p(x))$ then $\varphi_{0}(x)=\varphi(x)$ and is thus not a square. In any case, we are only concerned with $\varphi_{0}(x)$ being a square or not in $K(x)$. If $\varphi_{0}(x)$ is not a square then by the above, $m_{y, F}(X)$ does not split in $\widehat{K(x)}$. So from Theorem 3.4.7, we know that there is a unique absolute value $\|_{P^{\prime}}$ extending $\|_{P}$ given by $|z|_{P^{\prime}}=\left|N_{F^{\prime} / F}(z)\right|_{P}^{1 / 2}$ for all $z \in K(x, y)$. Therefore there is a unique place $P^{\prime} \mid P$ given by

$$
P^{\prime}=\left\{\left.z \in K(x, y)|| N_{F^{\prime} / F}(z)\right|_{P} ^{1 / 2}<1\right\}
$$

If $\varphi(x)_{0}$ is a square in $K(x)$, then by recursively solving the equations $(3.24),(3.25), \ldots$, we may find an element $a \in \widehat{K(x)}$ such that $m_{y, F}(X)=(X-a)(X+a)$. As such, $m_{y, F}(X)$ splits in $\widehat{K(x)}$. So from Example 3.4.7 we know that there are two distinct 
absolute value $\|_{P_{1}^{\prime}}$ and $\|_{P_{2}^{\prime}}$ extending $\|_{P}$ given by

$$
|g+h y|_{P_{1}^{\prime}}=|g+h a|_{\hat{P}} \quad \text { and } \quad|g+h y|_{P_{2}^{\prime}}=|g-h a|_{\hat{P}}
$$

for all $g+h y \in K(x, y)$. Therefore, there are exactly two distinct places $P_{1}^{\prime}$ and $P_{2}^{\prime}$ lying over $P$ given by

$$
\begin{aligned}
& P_{1}^{\prime}=\left\{g+h y \in K(x, y)|| g+\left.h a\right|_{\hat{P}}<1\right\}, \\
& P_{2}^{\prime}=\left\{g+h y \in K(x, y)|| g-\left.h a\right|_{\hat{P}}<1\right\} .
\end{aligned}
$$

Case 3. $P=P_{\infty}$ where $\operatorname{deg}(\varphi(x))$ is odd:

Since $\operatorname{deg}(\varphi(x))$ is odd, $v_{P}(\varphi(x))=-\operatorname{deg}(\varphi(x))$ is odd. Thus, by the above, $m_{y, F}(X)$ does not split in $\widehat{K(x)}$. From Theorem 3.4.7 we then know that there is a unique absolute value $\|_{P^{\prime}}$ extending $\|_{P_{\infty}}$ given by $|z|_{P^{\prime}}=\left|N_{F^{\prime} / F}(z)\right|_{P_{\infty}}^{1 / 2}$ for all $z \in K(x, y)$. Therefore there is a unique place $P^{\prime} \mid P$ given by

$$
P^{\prime}=\left\{\left.z \in K(x, y)|| N_{F^{\prime} / F}(z)\right|_{P_{\infty}} ^{1 / 2}<1\right\}
$$

Case 4. $P=P_{\infty}$ where $\operatorname{deg}(\varphi(x))$ is even:

Since $\operatorname{deg}(\varphi(x))$ is even we know that $v_{P}(\varphi(x))=-\operatorname{deg}(\varphi(x))$ is even. So, in the above notation, $m=-\operatorname{deg}(\varphi(x))$. Now we need to check if $\varphi_{m}(x)$ is a square in $K(x)$. From Example 3.1.16 we know that $x^{-1}$ is a local parameter at $P_{\infty}$. By Corollary 3.2.22 we may uniquely express $\varphi(x)$ as a Laurent series over $K$ in $x^{-1}$ as follows.

$$
\varphi(x)=\sum_{i=0}^{\operatorname{deg}(\varphi(x))} \varphi_{i} \cdot(x)^{i}=\sum_{i=0}^{-m} \varphi_{i} \cdot\left(x^{-1}\right)^{-i}=\sum_{i=m}^{0} \varphi_{-i} \cdot\left(x^{-1}\right)^{i} .
$$

We see that the coefficient $\varphi_{m}$ is just the leading coefficient of $\varphi(x)$. Thus, we are interested in whether the leading coefficient of $\varphi(x)$ is a square or not in $K(x)$. If it is not a square, then by the above, $m_{y, F}(X)$ does not split in $\widehat{K(x)}$. From Theorem 
3.4.7 we then know that there is a unique absolute value \|\|$_{P^{\prime}}$ extending \|\|$_{P_{\infty}}$ given by $|z|_{P^{\prime}}=\left|N_{F^{\prime} / F}(z)\right|_{P_{\infty}}^{1 / 2}$ for all $z \in K(x, y)$. Therefore there is a unique place $P^{\prime} \mid P_{\infty}$ given by

$$
P^{\prime}=\left\{\left.z \in K(x, y)|| N_{F^{\prime} / F}(z)\right|_{P_{\infty}} ^{1 / 2}<1\right\} .
$$

If $\varphi(x)$ is a square in $K(x)$ then by recursively solving the equations (3.24),(3.25), $\ldots$, we may find an element $a \in \widehat{K(x)}$ such that $m_{y, F}(X)=(X-a)(X+a)$. As such, $m_{y, F}(X)$ splits in $\widehat{K(x)}$. From Theorem 3.4 .7 we then know that there are two distinct absolute value $\|_{P_{1}^{\prime}}$ and $\|_{P_{2}^{\prime}}$ extending $\|_{P_{\infty}}$ given by

$$
|g+h y|_{P_{1}^{\prime}}=|g+h a|_{\hat{P}_{\infty}} \quad \text { and } \quad|g+h y|_{P_{2}^{\prime}}=|g-h a|_{\hat{P}_{\infty}}
$$

for all $g+h y \in K(x, y)$. Therefore there are exactly two distinct places $P_{1}^{\prime}$ and $P_{2}^{\prime}$ lying over $P_{\infty}$ given by

$$
\begin{gathered}
P_{1}^{\prime}=\left\{g+h y \in K(x, y)|| g+\left.h a\right|_{\hat{P}}<1\right\} \\
P_{2}^{\prime}=\left\{g+h y \in K(x, y)|| g-\left.h a\right|_{\hat{P}}<1\right\} .
\end{gathered}
$$

By Theorem 3.1.20, this exhausts all possible places of $K(x)$ and consequently all possible places of $K(x, y)$.

\subsection{Further Properties of the Ramification Index and Relative Degree}

Our goal in this section is to build up the tools to explicitly describe the ramification index and relative degree of the places of a quadratic extension of $K(x)$. Using properties of the Galois group, and completions, we are able to prove a fundamental theorem (Theorem 3.6.7) which relates the ramification index, relative degree and the number of places lying over a given places of $K(x)$. We will utilize this theorem in 
the following section.

Lemma 3.6.1 Let $F^{\prime}=K(x, y)$ be a quadratic extension of $F=K(x)$ as in Lemma 3.3.2. Let $P \in \mathcal{P}_{F}$ and $P^{\prime} \in \mathcal{P}_{F^{\prime}}$. Then the Galois group of $F^{\prime} / F$ acts transitively on the places $P^{\prime} \mid P$.

Proof. Let $\sigma: K(X) \hookrightarrow \widehat{K(x)}$ be as in Proposition 3.4.5 and let $\tau$ be the non-trivial Galois automorphism of Proposition 3.3.18. Let $m_{y, K(x)}(X)=X^{2}-\varphi(x)$. There are two cases to consider.

1. Suppose that $\sigma\left(m_{y, K(x)}\right)$ does not split in $\widehat{K(x)}$. By Theorem 3.4.2, there is a unique absolute value $\|_{F^{\prime}}$ extending $\|_{F}$ given by

$$
|z|_{F^{\prime}}=\left|N_{F^{\prime} / F}(z)\right|_{F}^{1 / 2} \text { for all } z \in F^{\prime}
$$

Let $P^{\prime}=\left\{\left.z \in F^{\prime}|| z\right|_{F^{\prime}}<1\right\}$. Then by Lemma 3.3.13, $\tau\left(P^{\prime}\right)$ is a place such that $\tau\left(P^{\prime}\right) \mid P$. Since $P^{\prime}$ is the unique place such that $P^{\prime} \mid P, \tau\left(P^{\prime}\right)=P^{\prime}$. One may also see this by a direct computation.

2. Suppose that $\sigma\left(m_{y, K(x)}\right)$ splits in $\widehat{K(x)}$. Then by Theorem 3.4.7, there are exactly two places lying over $P$.

$$
\begin{gathered}
P_{-}^{\prime}=\left\{\alpha+\beta y \in F^{\prime}|| \alpha+\left.\beta(-\theta)\right|_{F}<1\right\} \\
\text { and } \\
P_{+}^{\prime}=\left\{\alpha+\beta y \in F^{\prime}|| \alpha+\left.\beta \theta\right|_{F}<1\right\}
\end{gathered}
$$

where $\theta \in \widehat{F}$ is a fixed root of $\sigma\left(m_{y, K(x)}\right)$. It is clear that $\tau\left(P_{+}^{\prime}\right)=P_{-}^{\prime}$ and $\tau\left(P_{-}^{\prime}\right)=P_{+}^{\prime}$.

Remark 3.6.2 Let $F^{\prime}=K(x, y)$ be an extension of $F=K(x)$ where $y^{2}=\varphi(x)$ is irreducible. Let $P \in \mathcal{P}_{F}$ and $P^{\prime} \in \mathcal{P}_{F^{\prime}}$. Now we know that the Galois group acts 
transitively on the places $P^{\prime} \mid P$. So utilizing Lemma 3.3 .13 we now also know that

$$
e\left(\tau\left(P^{\prime}\right) \mid P\right)=e\left(P^{\prime} \mid P\right) \quad \text { and } \quad f\left(\tau\left(P^{\prime}\right) \mid P\right)=f\left(P^{\prime} \mid P\right) .
$$

As such, $e\left(P^{\prime} \mid P\right)$ and $f\left(P^{\prime} \mid P\right)$ depend only on $P$. This is another reason we often just write $e$ and $f$ when $P$ is obvious.

We will define one more value on a place $P \in \mathcal{P}_{F}$.

Definition 3.6.3 Let $F^{\prime} / F$ be a extension of function fields as in Definition 3.3.1. Let $P$ be places of $F$. Then we define $r(P)$ to be the number of places $P^{\prime} \in \mathcal{P}_{F^{\prime}}$ such that $P^{\prime} \mid P$. We will often just write $r$ when the place $P$ is obvious.

Our next goal is to put strong upper bounds on the ramification index and relative degree of the places of a quadratic extension of $K(x)$.

Proposition 3.6.4 ([7] p.589) Let $F^{\prime} / F$ be a finite extension of function fields as in Definition 3.3.1. Let $P, P^{\prime}$ be places of $F$ and $F^{\prime}$ respectively, such that $P^{\prime} \mid P$. Then

$$
e \cdot f \leq n=\left[F^{\prime}: F\right] .
$$

Proof. To simplify notation, let $\|=\|_{P^{\prime}}$. Since $\|_{P^{\prime}}$ and $\|_{P}$ are equivalent on $F$ we just use the one $\|$ to represent the absolute value in all instances. Choose $u_{1}, \ldots, u_{f} \in$ $\mathcal{O}_{P^{\prime}}$ such that $\left\{u_{1}+P^{\prime}, \ldots, u_{f}+P^{\prime}\right\} \subset \mathcal{O}_{P^{\prime}} / P^{\prime}$ forms a basis of $\mathcal{O}_{P^{\prime}} / P^{\prime}$ as a vector space over $\mathcal{O}_{P} / P$. Observe that if $a_{i} \in \mathcal{O}_{P}$ then

$$
\sum_{i=1}^{f} a_{i} u_{i} \in P^{\prime} \quad \Rightarrow \quad a_{i} \in P \text { for all } 1 \leq i \leq f
$$

Next, consider the quotient group $\left|F^{\prime \times}\right| /\left|F^{\times}\right|$and recall from Proposition 3.3.12 that it has $e$ distinct elements. Let $b_{1}, \ldots, b_{e}$ be elements of $F^{\prime \times}$ such that

$$
\left|F^{\prime \times}\right| /\left|F^{\times}\right|=\left\{\left|b_{1}\right|\left|F^{\times}\right|, \ldots,\left|b_{e}\right|\left|F^{\times}\right|\right\} .
$$


Let $m=\max \left\{\left|b_{1}\right|, \ldots\left|b_{e}\right|\right\}$. Let $t \in F$ be a local parameter at $P$ as in Definition 3.1.15. Then there exists $k>0$ such that $\left|t^{k}\right|<1 / m$. Notice that $\left|t^{k} b_{1}\right|\left|F^{\times}\right|=\left|b_{1}\right|\left|F^{\times}\right|$and $\left|t^{k} b_{i}\right|<1$ for all $1 \leq i \leq e$. Therefore multiplying the $b_{i}$ 's by element $t^{k} \in P \subset F^{\times}$, we may assume that $\left\{b_{1}, \ldots, b_{e}\right\} \subset P^{\prime}$.

Our intention is to create a list of $e f$ elements of $F^{\prime}$ which are linearly independent over $F$. This would then give us that ef $\leq\left[F^{\prime}: F\right]$.

We shall first show that if $a_{i} \in F$ for all $1 \leq i \leq f$ then

$$
\sum_{i=1}^{f} a_{i} u_{i} \neq 0 \Rightarrow\left|\sum_{i=1}^{f} a_{i} u_{i}\right| \in\left|F^{\times}\right|
$$

If $\sum_{i=1}^{f} a_{i} u_{i} \neq 0$ then there exists some $1 \leq j \leq f$ such that $a_{j} \neq 0$. By re-indexing we may assume without loss of generality that $\left|a_{1}\right| \neq 0$ and $\left|a_{1}\right| \geq\left|a_{i}\right|$ for all $1 \leq i \leq f$. Then

$$
\left|\sum_{i=1}^{f} a_{i} u_{i}\right|=\left|a_{1}\right|\left|\sum_{i=1}^{f} \frac{a_{i}}{a_{1}} u_{i}\right| .
$$

Since $\left|a_{1}\right| \geq\left|a_{i}\right|$, and because $u_{i} \in \mathcal{O}_{P^{\prime}}$ implies that $\left|u_{i}\right| \leq 1$, we know that $\left|\sum_{i=1}^{f} \frac{a_{i}}{a_{1}} u_{i}\right| \leq 1$. Now, assume that

$$
\left|\sum_{i=1}^{f} \frac{a_{i}}{a_{1}} u_{i}\right|<1 .
$$

This implies that $\sum_{i=1}^{f} \frac{a_{i}}{a_{1}} u_{i} \in P^{\prime}$. By (3.27), this implies that $\frac{a_{i}}{a_{1}} \in P$ for all $1 \leq i \leq f$. i.e $\left|\frac{a_{i}}{a_{1}}\right|<1$ for all $1 \leq i \leq f$. However, for $i=1$, $\left|\frac{a_{1}}{a_{1}}\right|=1$; a contradiction of (3.27). Therefore Equation (3.29) is false and

$$
\left|\sum_{i=1}^{f} \frac{a_{i}}{a_{1}} u_{i}\right|=1 .
$$

Multiplying both sides of (3.30) by $\left|a_{1}\right|$, we have that $\left|\sum_{i=1}^{f} a_{i} u_{i}\right|=\left|a_{1}\right| \in\left|F^{\times}\right|$.

We proceed to show that the ef elements of the set $\left\{u_{i} b_{j} \mid i=1, \ldots, f j=\right.$ 
$1, \ldots, e\} \subset F^{\prime}$, are linearly independent over $F$. Now suppose that we have the relation $\sum_{i, j} a_{i j} u_{i} b_{j}=0$ for some $a_{i j} \in F$. Then rearranging the summations we see that

$$
\sum_{i, j} a_{i j} u_{i} b_{j}=\left(\sum_{i} a_{i 1} u_{i}\right) b_{1}+\left(\sum_{i} a_{i 2} u_{i}\right) b_{2}+\ldots+\left(\sum_{i} a_{i e} u_{i}\right) b_{e}=0 .
$$

Assume that there exists some $d$ such that $\sum_{i} a_{i d} u_{i} \neq 0$. Let $d \in\{1, \ldots, e\}$ be such that $\left|\sum_{i} a_{i d} u_{i} b_{d}\right| \geq\left|\sum_{i} a_{i j} u_{i} b_{j}\right|$ for all $1 \leq j \leq e$. Then by the Strong Triangle Inequality (Example 3.2.10) we have that

$$
\begin{aligned}
0 & =\left(\sum_{i} a_{i 1} u_{i}\right) b_{1}+\left(\sum_{i} a_{i 2} u_{i}\right) b_{2}+\ldots+\left(\sum_{i} a_{i e} u_{i}\right) b_{e} \\
-\sum_{i} a_{i d} u_{i} b_{d} & =\sum_{j \neq d}\left(\sum_{i} a_{i j} u_{i} b_{j}\right) \\
\left|\sum_{i} a_{i d} u_{i} b_{d}\right| & \leq \max _{j \neq d}\left\{\left|\sum_{i} a_{i j} u_{i} b_{j}\right|\right\} .
\end{aligned}
$$

However since

$$
\left|\sum_{i} a_{i d} u_{i} b_{d}\right| \geq \max _{j \neq d}\left\{\left|\sum_{i} a_{i j} u_{i} b_{j}\right|\right\} .
$$

there must exist some $j \neq d$ such that

$$
\left|\sum_{i} a_{i d} u_{i} b_{d}\right|=\left|\sum_{i} a_{i j} u_{i} b_{j}\right|
$$

By re-indexing, we may assume without loss of generality that $\left|\sum_{i} a_{i 1} u_{i} b_{1}\right|=$ $\left|\sum_{i} a_{i 2} u_{i} b_{2}\right| \neq 0$. This implies that $\sum_{i} a_{i 1} u_{i} \neq 0$ and $\sum_{i} a_{i 2} u_{i} \neq 0$, hence $\left|\sum_{i} a_{i 1} u_{i}\right| \in\left|F^{\times}\right|$and $\left|\sum_{i} a_{i 2} u_{i}\right| \in\left|F^{\times}\right|$by (3.28). However this means that $\left|b_{1}\right|\left|F^{\times}\right|=\left|b_{2}\right|\left|F^{\times}\right|$which contradicts the choice of $\left\{b_{1}, \ldots, b_{e}\right\}$. Thus the relation $\sum_{i, j} a_{i j} u_{i} b_{j}=0$ implies that $\sum_{i} a_{i j} u_{i}=0$ for all $j=1, \ldots, e$.

Let $m=\max _{i, j}\left\{\left|a_{i j}\right|\right\}$ and let $m_{1}, m_{2}$ be such that $\left|a_{m_{1} m_{2}}\right|=\max _{i, j}\left\{\left|a_{i j}\right|\right\}$. Let $t$ 
be a local parameter at $P$ as in Definition 3.1.15. In the proof of Proposition 3.3.12 we saw that $|t|$ generates $\left|F^{\times}\right|$. As such, there exists $k>0$ such that $\left|t^{k}\right|=1 / m$. If we again multiply the $a_{i j}$ by $t^{k} \in P \subset F^{\times}$, we may assume that all the $a_{i j} \in \mathcal{O}_{P}$. Furthermore, by this process, if $a_{i j} \neq 0$ for some $1 \leq i \leq f, 1 \leq j \leq e$ then $a_{m_{1} m_{2}} \neq 0$. This implies that $\left|t^{k} a_{m_{1} m_{2}}\right|=1$, i.e. $t^{k} a_{m_{1} m_{2}} \in \mathcal{O}_{P} \backslash P$. This, however, contradicts the independence of $\left\{u_{1}, \ldots, u_{f}\right\}$. This proves the independence over $F$ of the $e f$ elements $u_{i} b_{j}$. This in turn implies that ef $\leq\left[F^{\prime}: F\right]$.

We will now sharpen the inequality of the previous theorem.

Proposition 3.6.5 ([7] p.589) Let $F^{\prime} / F$ be a finite extension of function fields as in Definition 3.3.1. Let $P, P^{\prime}$ be places of $F$ and $F^{\prime}$ respectively, such that $P^{\prime} \mid P$. Let $\hat{F}$ and $\hat{F}^{\prime}$ denote the completions of $F$ and $F^{\prime}$ with respect to $\|_{P}$ and $\|_{P^{\prime}}$ respectively. Then

$$
e \cdot f \leq \hat{n}=\left[\hat{F}^{\prime}: \hat{F}\right] .
$$

Proof. We first show that $\left|F^{\times}\right|=\left|\hat{F}^{\times}\right|$. Let $\hat{a} \in \hat{F}$. By Definition 3.2.19, $F$ is dense in $\hat{F}$ and so there exists an $a \in F$ such that $|a-\hat{a}|<|\hat{a}|$ or equivalently

$$
\left|\frac{a}{\hat{a}}-1\right|<1
$$

If $\left|\frac{a}{\hat{a}}\right| \neq 1$ then $\left|\frac{a}{\hat{a}}-1\right|=\max \left\{\left|\frac{a}{\hat{a}}\right|, 1\right\} \geq 1$ by the Strict Triangle Inequality. However, this contradicts equation (3.31), and as such $\left|\frac{a}{\hat{a}}\right|=1$, i.e. $|a|=|\hat{a}|$. Thus $\left|F^{\times}\right| \supseteq\left|\hat{F}^{\times}\right|$. Since $F^{\times} \subseteq \hat{F}^{\times}$, we also have that $\left|F^{\times}\right| \subseteq\left|\hat{F}^{\times}\right|$and hence we have equality. A similar argument shows that $\left|F^{\prime \times}\right|=\left|\hat{F}^{\prime \times}\right|$ as well. Consequently

$$
\left[\left|\hat{F}^{\prime \times}\right|:\left|\hat{F}^{\times}\right|\right]=\left[\left|F^{\prime \times}\right|:\left|F^{\times}\right|\right]=e
$$

Now, let $\hat{\mathcal{O}}_{P^{\prime}}$ and $\hat{\mathcal{O}}_{P}$ be valuation rings of $\hat{F}^{\prime}$ and $\hat{F}$ respectively, and $\hat{P}^{\prime}$ and $\hat{P}$ be their corresponding maximal ideals. According to Proposition 3.3.6

$$
\mathcal{O}_{P^{\prime}}^{\prime}=\hat{\mathcal{O}}_{P^{\prime}}^{\prime} \cap F^{\prime} \quad \mathcal{O}_{P}=\hat{\mathcal{O}}_{P} \cap F \quad P^{\prime}=\hat{P}^{\prime} \cap F^{\prime} \quad P=\hat{P} \cap F
$$


Let $\sigma: F \rightarrow \hat{F}$ be the natural embedding of Proposition 3.4.5. Consider the map $\sigma^{\prime}: \mathcal{O}_{P} \rightarrow \hat{\mathcal{O}}_{P} / \hat{P}$ defined by $\sigma^{\prime}(z)=\sigma(z)+\hat{P}$. Since $|z|_{\hat{P}}=|z|_{P}$ for all $z \in F$ we have that $\sigma\left(\mathcal{O}_{P}\right) \subset \hat{\mathcal{O}}_{P}$, and thus $\sigma^{\prime}$ does indeed map to $\hat{\mathcal{O}}_{P} / \hat{P}$. We also have that $\sigma(P) \subset \hat{P}$ and thus $\operatorname{ker}\left(\sigma^{\prime}\right)=P$. Therefore $\sigma^{\prime}: \mathcal{O}_{P} / P \rightarrow \hat{\mathcal{O}}_{P} / \hat{P}$ is one to one. To prove that it is onto, let $\hat{a} \in \hat{\mathcal{O}}_{P}^{*}$. Since $F$ is dense in $\hat{F}$, there exists an $a \in F$ such that $|\hat{a}-a|<1$. This implies that $\hat{a}-a \in \hat{P}$ and thus $\hat{a}+\hat{P}=a+\hat{P}$. From Equation 3.31 we know that $|\hat{a}|=|a|=1$. Hence there exists and $a \in \mathcal{O}_{P} / P$ such that $\sigma^{\prime}(a)=\hat{a}+\hat{P}$. This being said, we see that we have an isomorphism

$$
a+P \mapsto a+\hat{P} .
$$

of the residue field $F_{P}=\mathcal{O}_{P} / P$ onto the residue field $\hat{F}_{P}=\hat{\mathcal{O}}_{P} / \hat{P}$. Similarly, we have a natural isomorphism $b+P^{\prime} \mapsto b+\hat{P}^{\prime}$ of the residue field $F_{P^{\prime}}^{\prime}=\mathcal{O}_{P^{\prime}}^{\prime} / P^{\prime}$ onto $\hat{F}_{P^{\prime}}^{\prime}=\hat{\mathcal{O}}_{P^{\prime}}^{\prime} / \hat{P}^{\prime}$. This yields a canonical isomorphism of vector spaces $F_{P}^{\prime} / F_{P}$ onto $\hat{F}_{P}^{\prime} / \hat{F}_{P}$. Hence $\left[F_{P}^{\prime}: F_{P}\right]=\left[\hat{F}_{P}^{\prime}: \hat{F}_{P}\right]=f$.

In conclusion, the ramification index and the relative degree are unchanged when passing to the completion. Define $n=\left[F^{\prime}: F\right]$ and $\hat{n}=\left[\hat{F}^{\prime}: \hat{F}\right]$. By combining Corollary 3.4.4 with the fact that $\hat{n} \leq n$ we have the sharper inequality

$$
e f \leq \hat{n} \leq n
$$

Let $F^{\prime} / F$ be a quadratic extension of function fields as in Definition 3.3.1. Let $P, P^{\prime}$ be places of $F$ and $F^{\prime}$ respectively, such that $P^{\prime} \mid P$. By Proposition 3.6.4 we know that ef $\leq n=2$ and so there are three possibilities for the values of $e$ and $f$, namely

1. $e=2$ and $f=1$

2. $e=1$ and $f=2$ 
3. $e=f=1$

For cases 1 and 2, by Proposition 3.6.5, $\hat{n}=2$. For case 3, nothing discussed so far tells us whether $\hat{n}=1$ or $\hat{n}=2$. Hence we have following proposition.

Proposition 3.6.6 Let $F^{\prime} / F$ be a quadratic extension as in Definition 3.3.1. Let $P, P^{\prime}$ be places of $F$ and $F^{\prime}$ respectively, such that $P^{\prime} \mid P$ and $e=f=1$. Then ef $=\left[\hat{F}^{\prime}: \hat{F}\right]=1$.

Proof. We first show that $\hat{\mathcal{O}}_{P^{\prime}}=\hat{\mathcal{O}}_{P}$ and the result will follow. Let $t$ be a local parameter of $\hat{\mathcal{O}}_{P}$ as in Definition 3.1.15. Since $v_{P}^{\prime}(t)=e \cdot v_{P}(t)=1$, as a result of Theorem 3.1.13, $t$ is a local parameter of $\hat{\mathcal{O}}_{P^{\prime}}$ as well. Let $z \in \hat{\mathcal{O}}_{P^{\prime}}$. Then there exists $k_{1} \geq 0$ such that $\left|z t^{-k_{1}}\right|=1$. Since $\left[\hat{\mathcal{O}}_{P^{\prime}} / \hat{P}^{\prime}: \hat{\mathcal{O}}_{P} / \hat{P}\right]=f=1$, there exists a $c_{\mathbf{1}} \in \hat{\mathcal{O}}_{P}$ such that $z t^{-k_{1}}+\hat{P}^{\prime}=c_{1}+\hat{P}^{\prime}$, i.e. $z-c_{1} t^{k_{1}} \in P^{\prime}$. Let

$$
z_{1}=z-c_{1} t^{k_{1}}
$$

We notice that $\left|z_{1}\right|=\left|z-c_{1} t^{k_{1}}\right|<|z|$. Repeating this process, there exists a $c_{2} \in \hat{\mathcal{O}}_{P}$ and $k_{2} \geq 0$ such that

$$
z_{2}=z_{1}-c_{2} t^{k_{2}} \in P^{\prime}
$$

As such,

$$
z=z_{2}+c_{1} t^{k_{1}}+c_{2} t^{k_{2}} \in P^{\prime}
$$

So by repeating this process $l$ times we get that

$$
z=z_{l}+\sum_{i=1}^{l} c_{i} t^{k_{i}} .
$$

As $l \rightarrow \infty$, we have that $|z|>\left|z_{1}\right|>\left|z_{2}\right|>\ldots>\left|z_{l}\right|$, and so $z_{l} \rightarrow 0$. This implies that

$$
z=\sum_{i=1}^{\infty} c_{i} t^{k_{i}} .
$$


Now, $z=\sum_{i=1}^{\infty} c_{i} t^{k_{i}} \in \hat{F}$, since $\hat{F}$ is complete (by Definition 3.2.19) and $z$ is the limit of the Cauchy sequence $\left\{\sum_{i=1}^{l} c_{i} t^{k_{i}}\right\}_{l=0}^{\infty}$. Since $z$ was chosen from $\hat{\mathcal{O}}_{P}^{\prime}$ we have shown that

$$
z=\sum_{i=1}^{\infty} c_{i} t^{k_{i}} \in \hat{F} \cap \hat{\mathcal{O}}_{P^{\prime}}=\hat{\mathcal{O}}_{P}
$$

by Proposition 3.3.6. This proves that $\hat{\mathcal{O}}_{P^{\prime}}=\hat{\mathcal{O}}_{P}$. Since $z$ or $z^{-1} \in \hat{\mathcal{O}}_{P^{\prime}}$ for all $z \in \hat{F}^{\prime}$, we have that $\hat{F}^{\prime}=\hat{F}$.

We now have all the tools to prove the following theorem.

Theorem 3.6.7 Let $F^{\prime} / F$ be a quadratic extension of function fields as in Definition 3.3.1. Let $P, P^{\prime}$ be places of $F$ and $F^{\prime}$ respectively. Then

$$
r \cdot e \cdot f=\sum_{P^{\prime} \mid P} e \cdot f=\left[F^{\prime}: F\right]=2
$$

Proof. By Proposition 3.6.4 we know that $e f \leq n=2$ and so there are three cases to consider.

1. $e=2$ and $f=1$

2. $e=1$ and $f=2$

3. $e=f=1$

For cases 1 and 2, by Proposition 3.6.5, $\hat{n}=2$. Thus, by Theorem 3.4.2, $\|_{P^{\prime}}$ is the unique absolute value on $\hat{F}^{\prime}$ extending $\|_{P}$ and thus the place $P^{\prime}$ is the unique place lying over $P$. For case 3 , we know by Proposition 3.6.6 that $\hat{n}=1$. From Theorem 3.4.7 we know that in this case there are exactly two distinct places lying over $P$. Moverover, from Remark 3.6.2, we know that $e=f=1$ for both places lying over $P$. Thus, in all cases the equality holds. 


\subsection{Further Properties of the Places of a Quadratic Extension of $K(x)$}

Using Theorem 3.6.7 we may now describe the relative degree and ramification indices of all the places of $K(x, y)$. As in Section 3.5, we devote a subsection to each type of place in $K(x)$. For each type of place, we examine the properties of all places lying over each. More specifically, let $F^{\prime}=K(x, y)$ be a quadratic extension of $F=K(x)$ as in Lemma 3.3.2. Let $m_{y, F}(X)=X^{2}-\varphi(x)$. Let $P$ be a place of $K(x)$. We will again break down our examination of $K(x, y)$ in to four different cases.

Case 1.P $=P_{p(x)}$ where $p(x) \mid \varphi(x)$ :

In Section 3.5 we showed that in this case there is a unique place $P^{\prime} \mid P$ given by

$$
P^{\prime}=\left\{\left.z \in K(x, y)|| N_{F^{\prime} / F}(z)\right|^{1 / 2}<1\right\}
$$

In this case $y^{2}=\varphi(x)=p(x) g(x)$ for some $g(x) \in K[x]$ where $p(x) \nmid g(x)$. We take the discrete valuation $v_{P^{\prime}}$ of both sides to obtain

$$
\begin{aligned}
v_{P}^{\prime}\left(y^{2}\right) & =v_{P}^{\prime}(\varphi(x)) \\
2 \cdot v_{P}^{\prime}(y) & =e \cdot v_{P}(\varphi(x)) \\
2 \cdot v_{P}^{\prime}(y) & =e \cdot 1 .
\end{aligned}
$$

Since $v_{P}^{\prime}(y)$ is an integer (Definition 3.2.1) and $e$ is a positive integer (Definition 3.3.11) we know that $e \geq 2$. However, by Proposition 3.6.4, $e \leq 2$. Therefore, in this case, $e=2$ and by Theorem 3.6.7, $f=1$. There is another matter to clarify. Let $z \in F$ then using the absolute value given in Section 3.5 we have that

$$
|z|_{P^{\prime}}=\left|N_{F^{\prime} / F}(z)\right|_{P}^{1 / 2}=\left|z^{2}\right|_{P}^{1 / 2}=|z|_{P} \neq|z|_{P}^{e}
$$


This is because Theorem 3.4.2, gives us the unique absolute value on $\hat{F}$ such that $|z|_{P^{\prime}}=|z|_{P}$ for all $z \in F$. Since equivalent absolute values define the same place, in this case we will take the absolute value of $P^{\prime}$ to be $|z|_{P^{\prime}}=\left|N_{F^{\prime} / F}(z)\right|_{P}$. So, in keeping with Propostion 3.3.12, we have that $|z|_{P^{t}}=|z|_{P}^{e}$.

Case 2. $P=P_{p(x)}$ where $p(x) \nmid \varphi(x)$ :

We know from Example 3.1.16 that $p(x)$ is a local parameter at $P$. Consequently, by Proposition 3.2.21, we may uniquely express $\varphi(x)$ as

$$
\varphi(x)=\sum_{i=0}^{k} \varphi_{i}(x) p(x)^{i}
$$

for some $k>0$ where $\operatorname{deg}\left(\varphi_{i}(x)\right)<\operatorname{deg}(p(x))$ for all $0 \leq i \leq k$. If $\varphi_{0}(x)$ is not a square in $K(x)$ then we know from Section 3.5 that there is a unique place $P^{\prime} \mid P$ given by

$$
P^{\prime}=\left\{\left.z \in K(x, y)|| N_{F^{\prime} / F}(z)\right|_{P} ^{1 / 2}<1\right\} .
$$

Taking the discrete valuation of $P^{\prime}$ of $y^{2}=\varphi(x)$ we see that

$$
\begin{aligned}
v_{P}^{\prime}\left(y^{2}\right) & =v_{P}^{\prime}(\varphi(x)) \\
2 \cdot v_{P}^{\prime}(y) & =e \cdot v_{P}(\varphi(x)) \\
2 \cdot v_{P}^{\prime}(y) & =e \cdot 0,
\end{aligned}
$$

since $p(x) \nmid \varphi(x)$. As in Theorem 3.2.3, this means that $y$ is a unit of $\mathcal{O}_{P^{r}}$ and hence a non-trivial element of $F_{P^{\prime}} \backslash\left(\mathcal{O}_{P}+P^{\prime}\right) / P^{\prime}$. This implies that $F_{P^{\prime}}$ is a nontrivial extension of $F_{P}$. Equivalently, by Definition 3.3.9, $f=\left[F_{P^{\prime}}: F_{P}\right]>2$. However, by Proposition 3.6.4, $f \leq 2$, and thus $f=2$. So by Theorem 3.6.7 we also know that $e=1$.

If $\varphi_{0}$ is a square in $K(x)$ then in Section 3.5 we showed that there are exactly two 
distinct places $P_{1}^{\prime}$ and $P_{2}^{\prime}$ lying over $P$ given by

$$
\begin{gathered}
P_{1}^{\prime}=\left\{g+h y \in K(x, y)|| g+\left.h a\right|_{\hat{P}}<1\right\} \\
P_{2}^{\prime}=\left\{g+h y \in K(x, y)|| g-\left.h a\right|_{\hat{P}}<1\right\} .
\end{gathered}
$$

Since $r(P)=2$, by Theorem 3.6.7, we have that $e=f=1$.

Case 3. $P=P_{\infty}$ where $\operatorname{deg}(\varphi(x))$ is odd:

In Section 3.5 we showed that in this case there is a unique place $P^{\prime} \mid P$ given by

$$
P^{\prime}=\left\{\left.z \in K(x, y)|| N_{F^{\prime} / F}(z)\right|_{P_{\infty}} ^{1 / 2}<1\right\}
$$

Since $\operatorname{deg}(\varphi(x))$ is odd, $v_{P}(\varphi(x))=-\operatorname{deg}(\varphi(x))=2 k+1$ is odd. So again taking the valuation of $P^{\prime}$ of $y^{2}=\varphi(x)$ we have that

$$
\begin{aligned}
v_{P}^{\prime}\left(y^{2}\right) & =v_{P}^{\prime}(\varphi(x)) \\
2 \cdot v_{P}^{\prime}(y) & =e \cdot v_{P}(\varphi(x)) \\
2 \cdot v_{P}^{\prime}(y) & =e \cdot(2 k+1) .
\end{aligned}
$$

Since $v_{P}^{\prime}(y)$ is an integer, we see that $e$ must be even. However, by Proposition 3.6.4, $e \leq 2$. Therefore, in this case, $e=2$ and by Theorem 3.6.7, $f=1$. Again, in this case we will take the absolute value of $P^{\prime}$ to be $|z|_{P^{\prime}}=\left|N_{F^{\prime} / F}(z)\right|_{P}$. This way, we again will be in keeping with Propostion 3.3.12, which says that $|z|_{P^{\prime}}=|z|_{P}^{e}$.

Case 4. $P=P_{\infty}$ where $\operatorname{deg}(\varphi(x))$ is even:

Since $\operatorname{deg}(\varphi(x))$ is even, we know that $v_{P}(\varphi(x))=-\operatorname{deg}(\varphi(x))=2 k$. If the leading coefficient of $\varphi(x)$ is not a square in $K(x)$ then from Section 3.5, we know that there 
is a unique place $P^{\prime} \mid P_{\infty}$ given by

$$
P^{\prime}=\left\{\left.z \in K(x, y)|| N_{F^{\prime} / F}(z)\right|_{P_{\infty}} ^{1 / 2}<1\right\}
$$

Taking the discrete valuation of $P^{\prime}$ of $y^{2}=\varphi(x)$, we see that

$$
\begin{aligned}
v_{P}^{\prime}\left(y^{2}\right) & =v_{P}^{\prime}(\varphi(x)) \\
2 \cdot v_{P}^{\prime}(y) & =e \cdot v_{P}(\varphi(x)) \\
2 \cdot v_{P}^{\prime}(y) & =e \cdot(-2 k) \\
v_{P}^{\prime}(y) & =e(-k)
\end{aligned}
$$

If we consider the element $y x^{-k}$ then from Example 3.1.16 we know that $v_{P}\left(x^{-1}\right)=1$. Combining this with the above equation gives

$$
v_{P^{\prime}}\left(y x^{-k}\right)=v_{P^{\prime}}(y)+v_{P^{\prime}}\left(x^{-k}\right)=-e k+e v_{P}\left(x^{-k}\right)=0 .
$$

This means that $y x^{-k}$ is a unit of $\mathcal{O}_{P^{\prime}}$ and hence a non-trivial element of $F_{P^{\prime}} \backslash\left(\mathcal{O}_{P}+\right.$ $\left.P^{\prime}\right) / P^{\prime}$. The fact that $y x^{-k} \notin \mathcal{O}_{P}$ implies that $F_{P^{\prime}}$ is a nontrivial extension of $F_{P}$. Equivalently $f=\left[F_{P^{\prime}}: F_{P}\right]>2$. However, by Proposition 3.6.4, $f \leq 2$. Therefore, in this case, $f=2$ and by Theorem 3.6.7, $e=1$.

If the leading coefficient of $\varphi(x)$ is a square in $K(x)$ then as in Section 3.5 there are exactly two distinct places $P_{1}^{\prime}$ and $P_{2}^{\prime}$ lying over $P_{\infty}$ given by

$$
\begin{gathered}
P_{1}^{\prime}=\left\{g+h y \in K(x, y)|| g+\left.h a\right|_{\hat{P}}<1\right\} \\
P_{2}^{\prime}=\left\{g+h y \in K(x, y)|| g-\left.h a\right|_{\hat{P}}<1\right\} .
\end{gathered}
$$

Since there are two places lying over $P$, by Theorem 3.6 .7 we know that $e=f=1$. 


\subsection{Divisors}

Just as we did with points on a curve in Section 2.5, we define divisors on places of a function field. Again using valuations, we utilize divisors to record the zeros and poles of a rational function. Due to the similarities, most of our results are completely analogous to those of Section 2.5. We begin by defining a divisor of places.

Definition 3.8.1 ([19] p.14) Let $F$ be a function field. The additively written free abelian group which is generated by the places of $F$ is denoted by $\mathcal{D}_{F}$ and is called the divisor group of $F$. The elements of $\mathcal{D}_{F}$ are called divisors of $F$.

Again, a divisor may be written as a formal sum

$$
D:=\sum_{P \in \mathcal{P}_{F}} n_{P} P \quad \text { with } \quad n_{P} \in \mathbb{Z}
$$

with all but finitely many $n_{P}=0$. Two divisors are added term-wise, i.e. for $D, D^{\prime} \in$ $\mathcal{D}_{F}$

$$
D+D^{\prime}=\sum_{P \in \mathcal{P}_{F}}\left(n_{P}+n_{P^{\prime}}\right) P
$$

The definition of the support of a divisor of a function field is analogous to the one given for a curve (Defintion 2.5.2). Likewise, we define a partial ordering on the divisors of a function field as we did for divisors of a curve. The degree of a divisor is however slightly different.

Definition 3.8.2 The degree of a divisor $D \in \mathcal{D}_{F}$, denoted $\operatorname{deg}(D)$, is defined by

$$
\operatorname{deg}(D):=\sum_{P \in \mathcal{P}_{F}} n_{P} \cdot \operatorname{deg}(P)
$$

where $\operatorname{deg}(P)$ denotes the degree of the place $P$ as defined in Definition 3.1.17.

From here, we may construct divisors of elements of a function field. We will begin with the function field $K(x)$. Recall the definition of a zero and a pole of an element of a function field from Definition 3.2.5. 
Proposition 3.8.3 ([16] p.153) Let $z(x) \in K(x)$. Then $z(x)$ has finitely many zeros and poles.

Proof. Let $z(x) \in K(x)$ and so $z(x)$ may be written as $z(x)=\frac{a(x)}{b(x)}$ for some $a(x), b(x) \in K[x]$. We may assume without loss of generality that $a(x)$ and $b(x)$ have no common factors. Since $K[x]$ is a unique factorization domain,

$$
a(x)=\prod_{i=1}^{m} p_{i}(x)^{e_{i}} \quad b(x)=\prod_{j=1}^{n} q_{j}(x)^{f_{j}}
$$

for some $e_{i}, f_{j}>0$ and $q_{j}(x), p_{i}(x) \in K[x]$ distinct irreducible polynomials for all $1 \leq i \leq m, 1 \leq j \leq n$. By Corollary 3.2.6, for an irreducible polynomial $p(x) \in K[x]$,

$$
\begin{gathered}
v_{p(x)}(z)>0 \Leftrightarrow p(x)=p_{i}(x) \text { for some } 1 \leq i \leq m \\
v_{p(x)}(z)<0 \Leftrightarrow p(x)=q_{j}(x) \text { for some } 1 \leq j \leq n .
\end{gathered}
$$

Lastly, $v_{P_{\infty}}(z)=\operatorname{deg}(b(x))-\operatorname{deg}(a(x))$. By Theorem 3.1.20, these exhaust all the places of $K(x)$. Therefore $z$ has finitely many zeros and poles.

This allows us to construct well-defined divisors on elements of $K(x)$.

Definition 3.8.4 Let $0 \neq z \in K(x)$. Then the divisor of the function $z \in K(x)$ is denoted by $\operatorname{div}(z)$ and is defined as

$$
\operatorname{div}(z):=\sum_{P \in \mathcal{P}_{K(x)}} v_{P}(z) P
$$

A divisor $D$ is called principal if there exists a function $z \in K(x)$ such that $\operatorname{div}(z)=$ D.

Example 3.8.5 In this example we will assume that -1 is not a square in $K$. Let $g(x)=\frac{\left(x^{2}+1\right)(x+1)^{3}}{(x-1)^{2}} \in K(x)$. We see from Corollary 3.2.6 that

$$
v_{P_{x^{2}+1}}(g)=1 \quad v_{P_{-1}}(g)=3 \quad v_{P_{1}}(g)=-2 .
$$


At the infinite place of Theorem 3.1.20, we have that

$$
v_{P_{\infty}}(g)=\operatorname{deg}(x-1)^{2}-\operatorname{deg}\left(\left(x^{2}+1\right)(x+1)^{3}\right)=-3 .
$$

Clearly, we also see that $v_{P}(g)=0$ for all other $P \in \mathcal{P}_{F}$. So the zeros of $g$ are $P_{x^{2}+1}$ and $P_{-1}$ and the poles are $P_{1}$ and $P_{\infty}$. Furthermore, the principal divisor of $g$ is

$$
\operatorname{div}(g)=P_{x^{2}+1}+3 P_{-1}-2 P_{1}-3 P_{\infty}
$$

Checking the degree of $\operatorname{div}(g)$, as defined in Definition 3.8.2, we see that

$$
\begin{aligned}
\operatorname{deg}(\operatorname{div}(g)) & =1 \cdot \operatorname{deg}\left(P_{x^{2}+1}\right)+3 \cdot \operatorname{deg}\left(P_{-1}\right)-2 \cdot \operatorname{deg}\left(P_{1}\right)-3 \operatorname{deg}\left(P_{\infty}\right) \\
& =(1)(2)+(3)(1)-2(1)-3(1) \\
& =0
\end{aligned}
$$

We of course want to define divisors on quadratic extensions as well. Before we do that, we have to prove that they have finitely many zeros and poles. This requires the following lemma.

Lemma 3.8.6 Let $F^{\prime}=K(x, y)$ be a quadratic extension of $F=K(x)$ as in Lemma 3.3.2. Let $z \in K[x, y]$ be a polynomial. Let $P^{\prime}$ and $P$ be a places of $F^{\prime}$ and $F$ respectively such that $P^{\prime} \mid P$ and $P \neq P_{\infty}$. Then $v_{P^{\prime}}(z)>0$ implies that $v_{P}\left(N_{F^{\prime} / F}(z)\right)>0$.

Proof. Since $v_{P^{\prime}}(z) \geq 0$, by Theorem 3.2.3, $z \in \mathcal{O}_{P^{\prime}}$. Let $\tau$ be the non-trivial Galois automorphism from Proposition 3.3.18. Then $\tau(z)$ is also a polynomial and hence $\tau(z) \in \mathcal{O}_{P^{\prime}}$ as well (Section 3.5). Then $v_{P^{\prime}}(z)>0$ implies that

$$
v_{P}\left(N_{F^{\prime}} / F^{\prime}(z)\right)=v_{P}(z \tau(z))=1 / e \cdot v_{P^{\prime}}(z \tau(z))=1 / e\left(v_{P^{\prime}}(z)+v_{P^{\prime}}(\tau(z))\right)>0
$$

Theorem 3.8.7 ([9] p.167) Let $z \in K(x, y)$ where $K(x, y)$ is a quadratic extension 
of $K(x)$ as in Lemma 3.3.2. Then $z$ has finitely many zeros and poles in the sense of Definition 3.2.5.

Proof. Since $z \in K(x, y), z=\frac{a(x)+b(x) y}{c(x)}$ for some $a(x), b(x), c(x) \in K[x]$. Without loss of generality, we will assume that $a(x), b(x)$ and $c(x)$ have no common factors. Let $P^{\prime}$ be a place of $K(x, y)$ such that $P^{\prime}$ does not lie over $P_{\infty}$. By Lemma 3.8.6, if $a(x)+b(x) y$ has a zero at $P^{\prime}$ then $N_{K(x, y) / K(x)}(a(x)+b(x) y)$ has a zero at $P=P^{\prime} \cap$ $K(x)$. By Proposition 3.8.3, $N_{K(x, y) / K(x)}(a(x)+b(x) y)$ has only finitely many zeros. By Lemma 3.5.1, there are only finitely many places $P^{\prime} \mid P$. Therefore $a(x)+b(x) y$ has only finitely many zeros. Since the only other possible zeros of $z$ lie over $P_{\infty}$, and again by Lemma 3.5.1 there are only finitely many of those, $z$ has finitely many zeros. A similar argument on $c(x)$ shows that $z$ has finitely many poles.

Theorem 3.8.7 allows us to construct a well-defined divisors on elements of $K(x, y)$.

Definition 3.8.8 Let $K(x, y)$ be a quadratic extension of $K(x)$ as in Lemma 3.3.2. Let $0 \neq z \in K(x, y)$. Then the divisor of $z$ is denoted by $\operatorname{div}(z)$ and is defined as

$$
\operatorname{div}(z):=\sum_{P \in \mathcal{P}_{K(x, y)}} v_{P}(z) P
$$

A divisor $D$ is called principal if there exists a function $z \in K(x, y)$ such that $\operatorname{div}(z)=$ $D$.

Example 3.8.9 In this example we will let $K=\mathbb{Q}$. Let $\mathbb{Q}(x, y)$ be the quadratic extension of $\mathbb{Q}(x)$ defined by $y^{2}=\varphi(x)=-1 / 2\left(x^{2}+1\right)(x-2)$ Let $g=\frac{\left(x^{2}+1\right) y}{(x-1)^{2}} \in$ $\mathbb{Q}(x, y)$. We will now use Section 3.7 to calculate the non-zero valuations on $g$.

Since $\left(x^{2}+1\right)$ divides $\varphi(x)$ we know from Section 3.5 that there exists a unique place of $\mathbb{Q}(x, y)$, which we will denote as $P_{x^{2}+1}^{\prime}$, such that $P_{x^{2}+1}^{\prime} \mid P_{x^{2}+1}$. Moreover, we 
showed in Section 3.7 that this places is ramified, so

$$
\begin{aligned}
v_{x^{2}+1}^{\prime}(g) & =v_{P_{x^{2}+1}^{\prime}}\left(x^{2}+1\right)+v_{P_{x^{2}+1}^{\prime}}(y)-2 v_{P_{x^{2}+1}^{\prime}}(x-1) \\
& =2 v_{P_{x^{2}+1}}\left(x^{2}+1\right)+v_{P_{x^{2}+1}}\left(y^{2}\right)-0 \\
& =2+1=3 .
\end{aligned}
$$

Similarly, there is one place $P_{x-2}^{\prime} \mid P_{x-2}$ so we have that

$$
\begin{aligned}
v_{P_{x-2}^{\prime}}(g) & =v_{P_{x-2}^{\prime}}\left(x^{2}+1\right)+v_{P_{x-2}^{\prime}}(y)-2 v_{P_{x-2}^{\prime}}(x-1) \\
& =0+v_{P_{x-2}}\left(y^{2}\right)-0 \\
& =1 .
\end{aligned}
$$

Since $\varphi(1)=1$ is a square in $\mathbb{Q}(x)$, we know from Section 3.5 that there are two distinct places, which we will denote as $P_{1,-1}^{\prime}$ and $P_{1,1}^{\prime}$, that lie over $P_{x-1}$ Furthermore, in Section 3.7 we showed that these places are not ramified, so

$$
v_{P_{1,1}^{\prime}}(g)=v_{P_{1,1}^{\prime}}\left((x-1)^{-2}\right)=-2 v_{P_{1}}(x-1)=-2
$$

and similarly $v_{P_{1,-1}^{\prime}}(g)=-2$. Lastly, since $\operatorname{deg}(\varphi(x))=3$ is odd, we know from Section 3.5 there is a unique place $P_{\infty}^{\prime}$ that lies over $P_{\infty}$. We know from Section 3.7 that it is ramified, so

$$
\begin{aligned}
v_{P_{\infty}^{\prime}}(g) & =v_{P_{\infty}}\left(N_{\mathbb{Q}(x, y) / \mathbb{Q}(x)}(g)\right) \\
& =v_{P_{\infty}}\left(\frac{\left(x^{2}+1\right) y}{(x-1)^{2}} \frac{\left(x^{2}+1\right)(-y)}{(x-1)^{2}}\right) \\
& =v_{P_{\infty}}\left(\frac{\left(x^{2}+1\right)^{2} \varphi(x)}{(x-1)^{4}}\right) \\
& =4-7=-3 .
\end{aligned}
$$

One may also verify that $v_{P^{\prime}}(g)=0$ for all other $P \in \mathcal{P}_{\mathbb{Q}(x, y)}$. This gives us that the 
principal divisor of $g$ is

$$
\operatorname{div}(g)=3 P_{x^{2}+1}^{\prime}+1 P_{2}^{\prime}-2 P_{1,1}-2 P_{1,-1}-3 P_{\infty}
$$

From Section 3.7 we know all places in the support of $\operatorname{div}(g)$ have relative degree $f=1$, thus by Definition 3.3.9 their degrees are the same as the places they lie over. Hence, by Definition 3.8.2, the degree of the divisor of $g$ is

$$
\operatorname{deg}(\operatorname{div}(g))=3(2)+1(1)+1(1)-2(1)-2(1)-3(1)=7-7=0 .
$$

The principal divisors form a subgroup of the divisor group $\mathcal{D}_{F}$. It is denoted as $\mathcal{P}_{F}$.

Definition 3.8.10 Let $F$ be a function field. The quotient group $\mathcal{D}_{F} / \mathcal{P}_{F}$ is called the divisor class group of $F$. Two divisors $D_{1}, D_{2} \in \mathcal{D}_{F}$ are called equivalent if they are elements of the same coset of the divisor class group, i.e. if there exists a $z \in F$ such that $D_{1}=D_{2}+\operatorname{div}(z)$. In this case we write $D_{1} \sim D_{2}$

We will now demonstrate an important property of principal divisors.

Theorem 3.8.11 Let $z \in K(x)$. Then $\operatorname{deg}(\operatorname{div}(z))=0$.

Proof. Continuing from the proof of Proposition 3.8.3,

$$
z=\frac{a(x)}{b(x)}=\frac{\prod_{i=1}^{m} p_{i}(x)^{e_{i}}}{\prod_{j=1}^{n} q_{j}(x)^{f_{j}}}
$$

for some $e_{i}, f_{j}>0$ and $q_{j}(x), p_{i}(x) \in K[x]$ distinct irreducible polynomials for all $1 \leq i \leq m 1 \leq j \leq n$. Let $d_{a}=\operatorname{deg}(a(x))$ and $d_{b}=\operatorname{deg}(b(x))$. Then

$$
d_{a}=\sum_{i=1}^{m} e_{i} \cdot \operatorname{deg}\left(p_{i}(x)\right) \quad d_{b}=\sum_{j=1}^{n} f_{j} \cdot \operatorname{deg}\left(q_{j}(x)\right)
$$


Then by Corollary 3.2.6,

$$
\operatorname{div}(z)=\sum_{i=1}^{m} e_{i} P_{p_{i}(x)}-\sum_{j=1}^{n} f_{j} P_{q_{j}(x)}+\left(d_{b}-d_{a}\right) P_{\infty}
$$

Taking the degree of both sides, and recalling from Example 3.1.19 that $\operatorname{deg}\left(P_{p_{i}(x)}\right)=$ $\operatorname{deg}\left(p_{i}(x)\right)$, we see that

$$
\begin{aligned}
& \operatorname{deg}(\operatorname{div}(z))=\sum_{i=1}^{m} e_{i} \operatorname{deg}\left(P_{p_{i}(x)}\right)-\sum_{j=1}^{n} f_{j} \operatorname{deg}\left(P_{q_{j}(x)}\right)+\left(d_{b}-d_{a}\right) \operatorname{deg}\left(P_{\infty}\right) \\
& \operatorname{deg}(\operatorname{div}(z))=\sum_{i=1}^{m} e_{i} \operatorname{deg}\left(p_{i}(x)\right)-\sum_{j=1}^{n} f_{j} \operatorname{deg}\left(q_{j}(x)\right)+\left(d_{b}-d_{a}\right) .
\end{aligned}
$$

However, from (3.33), we see the the right hand side is 0 .

It is also true that the divisor of an element a quadratic extension of $K(x)$ has degree zero as well. To prove this we will need to make us of the results of next section.

Definition 3.8.12 Let $F$ be a function field. A element $z \in F$ is called regular if $\operatorname{div}(z) \geq 0$.

Example 3.8.13 Recall that a divisor $D=\sum_{P \in \mathcal{P}_{F}} n_{P} P \geq 0$ if and only if $n_{P} \geq 0$. Let $0 \neq z=\frac{a(x)}{b(x)} \in K(x)$ for some $a(x), b(x) \in K[x]$ be such that $d i v(z) \geq 0$. Again, we will assume without loss of generality that $a(x)$ and $b(x)$ have no common factors. If $b(x) \notin K$, then there exists an irreducible polynomial $p(x)$ such that $p(x) \mid g(x)$. This implies that $z$ will have a pole at $P_{p(x)}$ and thus not be regular. So $z$ must be a polynomial function. Now, if $z \notin K$ then $\operatorname{deg}(z)>0$ thus $v_{P_{\infty}}(z)=-\operatorname{deg}(z)<0$. This contradicts $\operatorname{div}(z) \geq 0$. Therefore, it must be the case that $z \in K$. i.e. the only regular functions in $K(x)$ are constant. A similar argument using the norm, as in the proof of Theorem 3.8.7, shows that the only regular elements in the quadratic extension $K(x, y)$ are constants as well. 
It would seem strange to define the term regular when the only regular functions are constant, except for the fact that this is also true about projective curves (Proposition 2.1.50). However, later on when we discuss differential forms, we will see that there may exist some non-constant regular elements. This was also the case for projective curves (Corollary 2.7.9).

\subsection{Divisors and Extensions: Norm and Conorm}

Through our work in Sections 3.5 and 3.7, we have related the places of $K(x, y)$ to those of $K(x)$. We will now look at ways relate the divisors of $K(x, y)$ to those of $K(x)$. We will use $\operatorname{div}_{F}(z)$ to denote a divisor in $F$ and $\operatorname{div}_{F^{\prime}}(z)$ to denote a divisor in $F^{\prime}$.

Definition 3.9.1 For a place $P \in \mathcal{P}_{F}$ we define the conorm of $P$ with respect to $F^{\prime} / F$ by

$$
\operatorname{Con}_{F^{\prime} / F}(P):=\sum_{P^{\prime} \mid P} e\left(P^{\prime} \mid P\right) P^{\prime}
$$

We may extend this definition to define the conorm of a divisor by

$$
\operatorname{Con}_{F^{\prime} / F}\left(\sum_{P \in \mathcal{P}_{F}} n_{P} P\right):=\sum_{P \in \mathcal{P}_{F}} n_{P} \cdot \operatorname{Con}_{F^{\prime} / F}(P) .
$$

Example 3.9.2 Following Section 3.5, we will demonstrate the possible conorms of the finite places of $K(x)$. Let $F=K(x)$ and $F^{\prime}=K(x, y)$ as in Lemma 3.3.2. Let $p(x)$ be an irreducible polynomial in $K[x]$, let us assume that $\operatorname{deg}(p(x))<\operatorname{deg}(\varphi(x))$.

1. In the event that $p(x) \nmid \varphi(x)$, we saw in Section 3.7 there were two cases for the possible places lying over $P_{p(x)}$.

(a) Recall from Section 3.5 if we divide $\varphi(x)$ by $p(x)$ and the remainder is square then there are two places $P_{1}^{\prime}$ and $P_{2}^{\prime}$ lying over $P_{p(x)}$. We also found in Section 3.7 that $e=f=1$ in both cases. By Definition 3.9.1, we have 
that

$$
\operatorname{Con}_{F^{\prime} / F}(P)=\sum_{P^{\prime} \mid P} e\left(P^{\prime} \mid P\right) P^{\prime}=P_{1}^{\prime}+P_{2}^{\prime} .
$$

(b) Recall from Section 3.7 that if we divide $\varphi(x)$ by $p(x)$ and the remainder is a square then there is a unique place $P^{\prime}$ lying over $P_{p(x)}$ which has relative degree $f=2$ and ramification index $e=1$. By Definition 3.9.1, we have that

$$
\operatorname{Con}_{F^{\prime} / F}(P)=\sum_{P^{\prime} \mid P} e\left(P^{\prime} \mid P\right) P^{\prime}=P^{\prime} .
$$

2. Recall from Section 3.7 that if $p(x) \mid \varphi(x)$ then there is a unique place $P^{\prime}$ lying over $P=P_{g(x)}$ which has relative degree $f=1$ and ramification index $e=2$. Again, by Definition 3.9.1 we have that

$$
\operatorname{Con}_{F^{\prime} / F}(P)=\sum_{P^{\prime} \mid P} e\left(P^{\prime} \mid P\right) P^{\prime}=2 P^{\prime} .
$$

By Theorem 3.6.7 we know that these three cases exhaust all possible types of places in $F^{\prime}$, lying over a place $P_{p(x)}$.

From the example above, it appears that there is a relation between the degrees of a divisor and its conorm. Indeed there is, and we shall describe it in the next proposition.

Proposition 3.9.3 Let $F^{\prime} / F$ be a finite extension of function fields as in Definition 3.3.1. Let $D=\sum_{P \in \mathcal{P}_{F}} n_{P} P \in \mathcal{D}_{F}$. Then

$$
\operatorname{deg}\left(\operatorname{Con}_{F^{\prime} / F}(D)\right)=\left[F^{\prime}: F\right] \operatorname{deg}(D) .
$$


Proof. We compute $\operatorname{deg}\left(\operatorname{Con}_{F^{\prime} / F}(D)\right)$ and find that

$$
\begin{aligned}
\operatorname{deg}\left(\operatorname{Con}_{F^{\prime} / F}(D)\right) & =\operatorname{deg}\left(\operatorname{Con}_{F^{\prime} / F}\left(\sum_{P \in \mathcal{P}_{F}} n_{P} P\right)\right) \\
& =\sum_{P \in \mathcal{P}_{F}} n_{P} \operatorname{deg}\left(\operatorname{Con}_{F^{\prime} / F}(P)\right) \\
& =\sum_{P \in \mathcal{P}_{F}} n_{P} \operatorname{deg}\left(\sum_{P^{\prime} \mid P} e\left(P^{\prime} \mid P\right) P^{\prime}\right) \\
& =\sum_{P \in \mathcal{P}_{F}} n_{P}\left(\sum_{P^{\prime} \mid P} e\left(P^{\prime} \mid P\right) \operatorname{deg}\left(P^{\prime}\right)\right) \\
& =\sum_{P \in \mathcal{P}_{F}} n_{P}\left(\sum_{P^{\prime} \mid P} e\left(P^{\prime} \mid P\right) f\left(P^{\prime} \mid P\right) \operatorname{deg}(P)\right) .
\end{aligned}
$$

By Theorem 3.6.7 we have that

$$
\begin{aligned}
\operatorname{deg}\left(\operatorname{Con}_{F^{\prime} / F}(D)\right) & =\sum_{P \in \mathcal{P}_{F}} n_{P}\left[F^{\prime}: F\right] \operatorname{deg}(P) \\
& =\left[F^{\prime}: F\right] \operatorname{deg}(D) .
\end{aligned}
$$

The previous proposition will prove especially useful in the next section. For now, we look at another map on divisors.

Definition 3.9.4 Let $F^{\prime} / F$ be a finite extension of function fields. For a place $P^{\prime} \in$ $\mathcal{P}_{F^{\prime}}$ lying over a place $P \in \mathcal{P}_{F}$ we define the norm of $P^{\prime}$ with respect to $F^{\prime} / F$ by

$$
N_{F^{\prime} / F}\left(P^{\prime}\right):=f\left(P^{\prime} \mid P\right) P .
$$

We may extend this definition to define the norm of a divisor by

$$
N_{F^{\prime} / F}\left(\sum_{P^{\prime} \in \mathcal{P}_{F^{\prime}}} n_{P^{\prime}} P^{\prime}\right):=\sum_{P^{\prime} \in \mathcal{P}_{F^{\prime}}} n_{P^{\prime}} \cdot N_{F^{\prime} / F}\left(P^{\prime}\right) .
$$


To understand the properties of the norm we return to Galois Theory (Subsection 3.3.4). It is of no coincidence that the Galois norm of Definition 3.3.17 and the norm of a divisor are both called norms. We will demonstrate their relationship in the following theorem.

Theorem 3.9.5 ([5] p.70) Let $F^{\prime}=K(x, y)$ be a quadratic extension of $F=K(x)$ as in Lemma 3.3.2. Then for all $z \in F^{\prime}$,

$$
N_{F^{\prime} / F}\left(\operatorname{div}_{F^{\prime}}(z)\right)=\operatorname{div}_{F}\left(N_{F^{\prime} / F}(z)\right) .
$$

Proof. Let $\tau$ be the non-trivial Galois automorphism of Proposition 3.3.18. By Definition 3.3.17, we see that

$$
\begin{aligned}
\operatorname{div}_{F}\left(N_{F^{\prime}}(z)\right) & =\sum_{P \in \mathcal{P}_{F}} v_{P}\left(N_{F^{\prime} / F^{\prime}}(z)\right) P \\
& =\sum_{P \in \mathcal{P}_{F}} v_{P}(z \cdot \sigma(z)) P \\
& =\sum_{P \in \mathcal{P}_{F}} \frac{1}{e} v_{P^{\prime}}(z \cdot \sigma(z)) P \quad \text { for any } P^{\prime} \mid P \\
& =\sum_{P^{\prime} \in \mathcal{P}_{F^{\prime}}} \frac{1}{e \cdot r} v_{P^{\prime}}(z \cdot \sigma(z)) P^{\prime} \\
& =\sum_{P^{\prime} \in \mathcal{P}_{F^{\prime}}} \frac{1}{e \cdot r} v_{P^{\prime}}(z) P^{\prime}+\sum_{P^{\prime} \in \mathcal{P}_{F^{\prime}}} \frac{1}{e \cdot r} v_{P^{\prime}}(\sigma(z)) P^{\prime} \\
& =\sum_{P^{\prime} \in \mathcal{P}_{F^{\prime}}} \frac{1}{e \cdot r} v_{P^{\prime}}(z) P^{\prime}+\sum_{P^{\prime} \in \mathcal{P}_{F^{\prime}}} \frac{1}{e \cdot r} v_{\sigma\left(P^{\prime}\right)}(z) P^{\prime}
\end{aligned}
$$

By Lemma 3.6.1, the group of Galois automorphisms acts transitively on the places of $P^{\prime} \mid P$. As such, the two summations of the final line are equal. Therefore, we have

$$
\operatorname{div}_{F}\left(N_{F^{\prime} / F}(z)\right)=\sum_{P^{\prime} \in \mathcal{P}_{F^{\prime}}} \frac{2}{e \cdot r} v_{P^{\prime}}(z) P^{\prime}=\sum_{P^{\prime} \in \mathcal{P}_{F^{\prime}}} \frac{\left[F^{\prime}: F\right]}{e \cdot r} v_{P^{\prime}}(z) P^{\prime}
$$


So by Theorem 3.6.7, we have that

$$
\operatorname{div}_{F}\left(N_{F^{\prime} / F}(z)\right)=\sum_{P^{\prime} \in \mathcal{P}_{F^{\prime}}} f P^{\prime} \cdot v_{P^{\prime}}(z)=N_{F^{\prime} / F}\left(\operatorname{div}_{F^{\prime}}(z)\right)
$$

as required.

Example 3.9.6 Following Section 3.5 we will demonstrate the possible norms of the finite places of $K(x, y)$. Let $F=K(x)$ and $F^{\prime}=K(x, y)$ be as in Lemma 3.3.2. Let $p(x)$ be an irreducible polynomial in $K[x]$ and let us assume that $\operatorname{deg}(p(x))<$ $\operatorname{deg}(\varphi(x))$.

1. In the event that $p(x) \nmid \varphi(x)$, we saw in Section 3.7 there were two cases for the possible places lying over $P_{p(x)}$.

(a) Recall from Section 3.5 if we divide $\varphi(x)$ by $p(x)$ and the remainder is square then there are two places $P_{1}^{\prime}$ and $P_{2}^{\prime}$ lying over $P_{p(x)}$. We also found in Section 3.7 that $e=f=1$ in both cases. By Definition 3.9.4 we have that

$$
N_{F^{\prime} / F}\left(P_{1}^{\prime}+P_{2}^{\prime}\right)=\sum_{P^{\prime} \mid P} f\left(P^{\prime} \mid P\right) P=2 P
$$

(b) Recall from Section 3.7 that if we divide $\varphi(x)$ by $p(x)$ and the remainder is a square then there is a unique place $P^{\prime}$ lying over $P_{p(x)}$ which has relative degree $f=2$ and ramification index $e=1$. By Definition 3.9.4 we have that

$$
N_{F^{\prime} / F}\left(P^{\prime}\right)=\sum_{P^{\prime} \mid P} f\left(P^{\prime} \mid P\right) P=2 P
$$

2. Recall from Section 3.7 that if $p(x) \mid \varphi(x)$ then there is a unique place $P^{\prime}$ lying over $P=P_{p(x)}$ which has relative degree $f=1$ and ramification index $e=2$. Again, by Definition 3.9.4 we have that

$$
N_{F^{\prime} / F}\left(P^{\prime}\right)=\sum_{P^{\prime} \mid P} f\left(P^{\prime} \mid P\right) P=P
$$


By Theorem 3.6.7 we know that these three cases exhaust all possible types of places in $F^{\prime}$ lying over a place $P_{p(x)}$.

The degree of a divisor and its norm are also strongly related.

Corollary 3.9.7 Let $F^{\prime}=K(x, y)$ be a quadratic extension of $F=K(x)$ as in Lemma 3.3.2. Then taking the norm does not change the degree of the divisor. Consequently,

$$
\operatorname{deg}\left(\operatorname{div}_{F^{\prime}}(z)\right)=\operatorname{deg}\left(N_{F^{\prime} / F}\left(\operatorname{div}_{F^{\prime}}(z)\right)\right)=\operatorname{deg}\left(\operatorname{div}_{F}\left(N_{F^{\prime} / F}(z)\right)\right) .
$$

Proof. By Theorem 3.9.5,

$$
N_{F^{\prime} / F^{F}}\left(\operatorname{div}_{F^{\prime}}(z)\right)=\operatorname{div}_{F}\left(N_{F^{\prime} / F}(z)\right) .
$$

So clearly,

$$
\operatorname{deg}\left(N_{F^{\prime} / F}\left(\operatorname{div}_{F^{\prime}}(z)\right)\right)=\operatorname{deg}\left(\operatorname{div}_{F}\left(N_{F^{\prime} / F}(z)\right)\right) .
$$

To prove the other equality, let $\operatorname{div}_{F^{\prime}}(z)=\sum_{P^{\prime} \in \mathcal{P}_{F^{\prime}}} v_{P^{\prime}}(z) P^{\prime}$. Then

$$
\begin{aligned}
\operatorname{deg}\left(\operatorname{div}_{F^{\prime}}(z)\right) & =\operatorname{deg}\left(\sum_{P^{\prime} \in \mathcal{P}_{F^{\prime}}} v_{P^{\prime}}(z) P^{\prime}\right) \\
& =\sum_{P^{\prime} \in \mathcal{P}_{F^{\prime}}} v_{P^{\prime}}(z) f\left(P^{\prime} \mid P\right) \operatorname{deg}(P) \\
& =\sum_{P^{\prime} \in \mathcal{P}_{F^{\prime}}} v_{P^{\prime}}(z) \operatorname{deg}\left(N_{F^{\prime} / F}\left(P^{\prime}\right)\right) \\
& =\operatorname{deg}\left(N_{F^{\prime} / F}\left(\sum_{P^{\prime} \in \mathcal{P}_{F^{\prime}}} v_{P^{\prime}}(z) P^{\prime}\right)\right) \\
& =\operatorname{deg}\left(N_{F^{\prime} / F}\left(\operatorname{div}_{F^{\prime}}(z)\right)\right)
\end{aligned}
$$

as required.

With the above theorem, we may now prove that in the case of a quadratic extension principal divisors have degree zero. 
Theorem 3.9.8 Let $F^{\prime}=K(x, y)$ be a quadratic extension of $F=K(x)$ as in Lemma 3.3.2. Let $z \in K(x, y)$. Then $\operatorname{deg}\left(\operatorname{div}_{F^{\prime}}(z)\right)=0$.

Proof. By Corollary 3.9.7, we have that

$$
\operatorname{deg}\left(\operatorname{div}_{F^{\prime}}(z)\right)=\operatorname{deg}\left(\operatorname{div}_{F}\left(N_{F^{\prime} / F}(z)\right)\right) .
$$

However, by Proposition 3.3.19, $N_{F^{\prime} / F}(z) \in K(x)$. As such, by Theorem 3.8.11,

$$
\operatorname{deg}\left(\operatorname{div}_{F}\left(N_{F^{\prime} / F}(z)\right)\right)=0
$$

Therefore, by Corollary 3.9.7, $\operatorname{deg}\left(\operatorname{div}_{F^{\prime}}(z)\right)=0$.

Example 3.9.9 Continuing from Example 3.8.9, we will let $K=\mathbb{Q}$. We let $\mathbb{Q}(x, y)$ be the quadratic extension of $\mathbb{Q}(x)$ defined by $y^{2}=\varphi(x)=-1 / 2\left(x^{2}+1\right)(x-2)$. We considered $g=\frac{\left(x^{2}+1\right) y}{(x-1)^{2}} \in K(x, y)$. We showed that the principal divisor of $g$ is

$$
\operatorname{div}(g)=3 P_{x^{2}+1}^{\prime}+1 P_{2}^{\prime}-2 P_{1,1}-2 P_{1,-1}-3 P_{\infty} .
$$

We also checked the degree of $\operatorname{div}(g)$ and saw that

$$
\operatorname{deg}(\operatorname{div}(g))=3(2)+1(1)+1(1)-2(1)-2(1)-3(1)=7-7=0
$$

Taking the norm of $g$, we get that

$$
N_{\mathbb{Q}(x, y) / \mathbb{Q}(x)}(g)=\frac{\left(x^{2}+1\right)^{2} y^{2}}{(x-1)^{4}}=\frac{\left(x^{2}+1\right)^{3}(x-2)}{-2(x-1)^{4}}
$$

Taking the divisor over $\mathbb{Q}(x)$ we see that

$$
\operatorname{div}_{F}\left(\left(N_{\mathbb{Q}(x, y) / \mathbb{Q}(x)}(g)\right)=3 P_{x^{2}+1}+P_{x-2}-4 P_{x-1}-3 P_{\infty}\right.
$$


which has degree 0 . Taking the norm of $\operatorname{div}_{\mathbb{Q}(x, y)}(g)$, we obtain that

$$
\begin{aligned}
& \left.N_{\mathbb{Q}(x, y) / \mathbb{Q}(x)} d i v_{\mathbb{Q}(x, y)}(g)\right) \\
= & 3\left(f\left(P_{x^{2}+1}\right)\right) P_{x^{2}+1}+f\left(P_{x-2}\right) P_{2}-2 f\left(P_{1}\right)\left(P_{1}+P_{1}\right)-3 f\left(P_{\infty}\right) P_{\infty} \\
= & 3 P_{x^{2}+1}+P_{x-2}-4 P_{x-1}-3 P_{\infty}
\end{aligned}
$$

as expected, and it too has degree 0.

There is also a nice result when combining the norm and conorm.

Proposition 3.9.10 Let $F^{\prime}=K(x, y)$ be a quadratic extension of $F=K(x)$. Let $z \in F$ then

$$
N_{F^{\prime} / F}\left(\operatorname{Con}_{F^{\prime} / F}\left(\operatorname{div}_{F}(z)\right)=\left[F^{\prime}: F\right] \operatorname{div}_{F}(z)\right.
$$

Proof. Let $P$ be a place of $F$. Then by Theorem 3.6.7,

$$
N_{F^{\prime} / F}\left(\operatorname{Con}_{F^{\prime} / F}(P)\right)=N_{F^{\prime} / F}\left(\sum_{P^{\prime} \mid P}(c) P^{\prime}\right)=\sum_{P^{\prime} \mid P}(f c) P=(\text { ref }) P=\left[F^{\prime}: F\right] P .
$$

Since the norm and conorm are $\mathbb{Z}$-linear functions on divisors, it follows that

$$
N_{F^{\prime \prime} / F}\left(\operatorname{Con}_{F^{\prime} / F}\left(\operatorname{div}_{F}(z)\right)\right)=\left[F^{\prime}: F\right] \operatorname{div}_{F}(z)
$$

\subsection{Differential Forms}

In this section we generalize the module of differential forms of a curve (Definition 2.6.21) to the module of differential forms over a function field. As we did in Chapter 2 , we define valuations and divisors of differential forms. We then give a definition of regularity of a differential form in terms of divisors. As was the case with curves, 
regular differential forms will provide us with a means to define the genus of a function field.

Definition 3.10.1 Let $F / K(x)$ be a function field. We define the module of rational differential forms of $F$ as the free $F$-module generated by the symbols $\left\{d_{F} z: z \in F\right\}$ modulo the following relations:

$$
\begin{gathered}
d_{F}(a+b)-d_{F}(a)-d_{F}(b) \text { for all } a, b \in F \\
d_{F}(a b)-a d_{F}(b)-d_{F}(a) b \text { for all } a, b \in F \\
d_{F}(k) \text { for all } k \in K .
\end{gathered}
$$

We will denote the module of rational differential forms of $F$ as $\Omega_{F}$.

We now define a derivation, a generalization of the $d$ function of Section 2.6.

Definition 3.10.2 ([19] p.134) Let $F$ be a function field and let $M$ be an $F$-module. A derivation is a map $D: F \rightarrow M$ with the following properties.

1. $D(a+b)=D(a)+D(b)$

2. $D(a b)=D(a) b+a D(b)$.

Just as we did in Chapter 2 we can consider $d_{F}$ as a map. Let $F / K(x)$ be a function field. It follows from Definition 3.10.1 that the map $d_{F}: F \rightarrow \Omega_{F}$ is a derivation where by $d_{F}(z)=d_{F} z$.

If we let $F^{\prime}$ be a finite extension of a function field $F$ as in Definition 3.3.1 then it is also clear that there is a natural embedding of $\Omega_{F}$ into $\Omega_{F^{\prime}}$. Indeed, $d_{F}(z)$ can be identified with $d_{F^{\prime}}(z)$ for all $z \in F$. So when we are only dealing with a field and its extensions we will take $d$ to be $d_{F^{\prime}}$.

Remark 3.10.3 Let $F^{\prime}$ be a quadratic extension of $K(x)$. Then just as in Proposition 
2.6.8, the $d$ map satisfies a chain rule, i.e.

$$
\begin{aligned}
d\left(a\left(b_{1}(x, y), b_{2}(x, y)\right)\right)= & \frac{\partial a}{\partial x}\left(b_{1}(x, y), b_{2}(x, y)\right) d\left(b_{1}(x, y)\right)+ \\
& \frac{\partial a}{\partial y}\left(b_{1}(x, y), b_{2}(x, y)\right) d\left(b_{2}(x, y)\right) .
\end{aligned}
$$

Proposition 3.10.4 $\Omega_{K(x)}$ is a rank one module over $K(x)$.

Proof. Let $u d z \in \Omega_{K}(x)$ where $u, z \in K(x)$. Since $z \in K(x)$ we have that $z=\frac{a(x)}{b(x)}$ for some $a(x), b(x) \in K[x]$. Since $d$ is a derivation,

$$
d z=\frac{a^{\prime}(x) b(x) d x-b^{\prime}(x) a(x) d x}{b(x)^{2}} .
$$

Therefore, $u d z=u \frac{a^{\prime}(x) b(x)-b^{\prime}(x) a(x)}{b(x)^{2}} d x$ and thus every differential form may be written as $v d x$ for some $v \in K(x)$. It follows that $d x$ is a basis of $\Omega_{K}(x)$, and hence $\Omega_{K}(x)$ is a rank one $K(x)$-module

Proposition 3.10.5 Let $F=K(x)$ and let $F^{\prime}$ be a finite extension of $K(x)$ as in Definition 3.3.1. Then $\Omega_{F^{\prime}}$ is a rank one $F^{\prime}$-module.

Proof. Using the fact that $F^{\prime} / F$ is algebraic, for all $0 \neq z \in F^{\prime}$, there exists $c_{i}(x) \in$ $K(x)$ such that

$$
c_{n}(x) z^{n}+c_{n-1}(x) z^{n-1}+\ldots+c_{1}(x) z+c_{0}(x)=0
$$

where $c_{n}(x) \neq 0$ and $c_{0}(x) \neq 0$. Taking $d$ of both sides,

$$
\left(c_{n}^{\prime}(x) d x z^{n}+(n-1) c_{n}(x) z^{n-1} d z\right)+\ldots+\left(c_{1}^{\prime}(x) d x+c_{1}(x) d z\right)+c_{0}^{\prime}(x) d x=0 .
$$

Rearranging the terms we have that,

$$
\begin{aligned}
& c_{n}^{\prime}(x) z^{n} d x+c_{n-1}^{\prime}(x) z^{n-1} d x+\ldots+c_{1}^{\prime}(x) d x+c_{0}^{\prime}(x) d x \\
= & c_{n}(x) z^{n-1} d z+(n-1) c_{n-1}(x) z^{n-1} d z+\ldots+c_{1}(x) d z .
\end{aligned}
$$


Since $c_{n}(x) \neq 0$, we may rearrange this equation to get that

$$
d z=\frac{c_{n}^{\prime}(x) z^{n}+c_{n-1}^{\prime}(x) z^{n-1}+\ldots+c_{1}^{\prime}(x)+c_{0}^{\prime}(x)}{c_{n}(x) z^{n-1}+(n-1) c_{n-1}(x) z^{n-1}+\ldots+c_{1}(x)} d x
$$

Therefore $d z=v d x$ for some $v \in F^{\prime}$. It follows from this that $d x$ is a basis of $\Omega_{F}^{\prime}$ and as such $\Omega_{F^{\prime}}$ is a rank one $F^{\prime}$-module.

Proposition 3.10.6 Let $F / K(x)$ be a function field. Let $P \in \mathcal{P}_{F}$ be a place of $F$, and let $t$ be a local parameter of $P$. Then for all $\omega \in \Omega_{F}, \omega=v d t$ for some $v$ in $F$.

Proof. By the definition of a function field (Definition 3.1.2), $F$ is a finite extension of $K(x)$. So by the proof of Propostion 3.10.5, $d x$ is a basis of $\Omega_{F}$. Let $\omega \in \Omega_{F}$. Then $\omega=w d x$ for some $w \in F$. Since $t \in P, t \notin K$ and so $0 \neq d t \in \Omega_{F}$ by Definition 3.10.2. Again, since $d x$ is a basis of $\Omega_{F}$ there exists some $z \in F$ such that $d t=z d x$. By combining these relations we see that $\omega=\frac{w}{z} d t$. Therefore $d t$ is also a basis of $\Omega_{F}$ and the result follows.

Definition 3.10.7 Let $F / K(x)$ be a function ficld. Let $P \in \mathcal{P}_{F}$ be a place of $F$. Let $t$ be a local parameter of $P$ and let $\omega \in \Omega_{F}$. By Proposition 3.10.6, $\omega$ may be written in the form $\omega=z \cdot d(t)$ with $z \in F$. Define the valuation at $P$ of a differential form to be $v_{P}(\omega)=v_{P}(z)$.

For a proof that this is well-defined for arbitrary function fields $F$ see [5] p.60. To show that the this definition is well-defined for the case of $[F: K(x)] \leq 2$, we make use of the following three lemmas.

Lemma 3.10.8 Let $P \in \mathcal{P}_{K(x)}$ and let $u$ be a unit of $\mathcal{O}_{P}$. Then $v_{P}(d u) \geq 0$.

Proof. Let $t(x)$ be a local parameter at $P$. From Corollary 3.2.22 and Corollary 3.2 .23 we know that $u$ may be uniquely written as

$$
u=\sum_{i=0}^{\infty} a_{i}(x) t(x)^{i}
$$


where $a_{i}(x)$ is a unit, $\operatorname{deg}\left(a_{i}\right)<\operatorname{deg}(P)$ and $a_{0}(x) \neq 0$. Taking $d$ of this equation we obtain that

$$
\begin{aligned}
d u & =\sum_{i=1}^{\infty} i a_{i}(x) t(x)^{i-1} d(t(x))+\sum_{i=0}^{\infty} t(x)^{i} d\left(a_{i}(x)\right) \\
& =\sum_{i=1}^{\infty} i a_{i}(x) t^{i-1} d(t(x))+\sum_{i=0}^{\infty} t(x)^{i} a_{i}^{\prime}(x) d x .
\end{aligned}
$$

Now, since $\operatorname{deg}\left(a_{i}^{\prime}(x)\right)<\operatorname{deg}(P)$ either $a_{i}(x) \in K$ in which case $a_{i}^{\prime}(x)=0$, or $a_{i}(x) \in$ $K[x]$ and $\operatorname{deg}\left(a_{i}(x)\right)>0$. By Theorem 3.1.20, this is only the case if $P=P_{p(x)}$ where $\operatorname{deg}(P)=\operatorname{deg}(p(x))<\operatorname{deg}(t(x))$ for some irreducible polynomial $p(x) \in K(x)$. From Example $3.1 .16, p(x)$ is also a local parameter at $P$. Since

$$
\operatorname{deg}\left(a_{i}^{\prime}(x)\right)<\operatorname{deg}(P)=\operatorname{deg}(p(x))
$$

$p(x) \nmid a_{i}^{\prime}(x)$ and thus $v_{P}\left(a_{i}^{\prime}(x)\right)=0$. In addition, computing that $d x=\frac{1}{t^{\prime}(x)} d(t(x))$, and since $t(x) \nmid t^{\prime}(x)$, by Definition 3.10.7,

$$
v_{P}(d x)=v_{P}\left(\frac{1}{t^{\prime}(x)} d(t(x))\right)=v_{P}\left(\frac{1}{t^{\prime}(x)}\right)=0 .
$$

Using this information, we take the valuation of (3.37) to obtain that

$$
\begin{aligned}
v_{P}(d u) & =v_{P}\left(\left(\sum_{i=1}^{\infty} i a_{i}(x) t(x)^{i-1}+\sum_{i=0}^{\infty} t(x)^{i} \frac{a_{i}^{\prime}(x)}{t^{\prime}(x)}\right) d(t(x))\right) . \\
& =v_{P}\left(\sum_{i=0}^{\infty}\left((i+1) a_{i+1}(x) t(x)^{i}+\frac{a_{i}^{\prime}(x)}{t^{\prime}(x)} t(x)^{i}\right)\right) .
\end{aligned}
$$

Now considering each term in the sum, we notice that

$$
\begin{aligned}
v_{P}\left((i+1) a_{i+1}(x) t(x)^{i}\right) & \left.=v_{P}(i+1)\right)+v_{P}\left(a_{i+1}(x)\right)+v_{P}\left(t(x)^{i}\right) \\
& =0+0+i \\
& \geq 0 \text { for all } i \geq 0,
\end{aligned}
$$


and

$$
\begin{aligned}
v_{P}\left(\frac{a_{i}^{\prime}(x)}{t^{\prime}(x)} t(x)^{i}\right) & =v_{P}\left(a_{i}^{\prime}(x)\right)-v_{P}\left(t^{\prime}(x)\right)+v_{P}\left(t(x)^{i}\right) \\
& =0+0+i \\
& \geq 0 \text { for all } i \geq 0 .
\end{aligned}
$$

Therefore

$$
v_{P}(d u) \geq \min _{i \geq 0}\left\{(i+1) a_{i+1}(x) t(x)^{i}, \frac{a_{i}^{\prime}(x)}{t^{\prime}(x)} t(x)^{i}\right\} \geq 0
$$

Lemma 3.10.9 Let $F^{\prime}$ be a function field extending $K(x)$ (Definition 3.3.1). Let $P^{\prime} \in \mathcal{P}_{F^{\prime}}$ and let $P$ be the place of $K(x)$ lying under $P^{\prime}$ (Corollary 3.3.7). Let $b_{1}, \ldots, b_{f} \in F^{\prime}$ be representatives of a basis of $F_{P^{\prime}} / F_{P}$. Let $z \in F^{\prime}$ and let $t^{\prime}$ be a local parameter of $P^{\prime}$. Then $z$ has a unique representation in the form

$$
z=\sum_{i=m}^{\infty}\left(\sum_{j=1}^{f} a_{i, j}(x) b_{j}\right) t^{\prime i} \quad \text { for some } m \in \mathbb{Z}
$$

where $a_{i, j}(x) \in K[x], \operatorname{deg}\left(a_{i, j}(x)\right)<\operatorname{deg}(P)$ and one of $a_{m, 1}(x), \ldots, a_{m, f}(x) \neq 0$.

Proof. From Example 3.1.19, $F_{P} \cong K[x] /(t(x))$ for some $t(x)$ where $\operatorname{deg}(t(x))=$ $\operatorname{deg}(P)$. Since $b_{1}, \ldots, b_{f}$ is a basis of $F_{P^{\prime}} / F_{P}$, the $a_{i}$ 's in the construction of the proof of Proposition 3.2.21 may be uniquely chosen as elements of the form $\sum_{j=1}^{f} a_{i, j}(x) b_{j}$ where $a_{i, j}(x) \in K[x]$, and $\operatorname{deg}\left(a_{i, j}(x)\right)<\operatorname{deg}(P)$

Lemma 3.10.10 Let $F^{\prime}$ be a function field such that $\left[F^{\prime}: K(x)\right] \leq 2$. Let $P^{\prime}$ be a place of $F^{\prime}$ and let $u$ be a unit of $\mathcal{O}_{P}$. Then $v_{P^{\prime}}(d(u)) \geq 0$.

Proof. The case that $[F: K(x)]=1$ is covered in Lemma 3.10.8. We assume without loss of generality that $F^{\prime}$ is a quadratic extension of $K(x)$ as in Lemma 3.3.2. As such $u$ will be written as $u(x, y)$. Let $P \in \mathcal{P}_{K(x)}$ be the place lying under $P^{\prime}$ (Corollary 
3.3.7). Let $t(x, y)$ be a local parameter at $P^{\prime}$. We consider two cases; $P=P_{p(x)}$ and $P=P_{\infty}$ as in Theorem 3.1.20.

1. For the case that $P=P_{p(x)}$. By Lemma 3.10 .9 we may express $u(x, y)$ as a Laurent series in terms of $t(x, y)$, i.e

$$
u(x, y)=\sum_{i=0}^{\infty} a_{i}(x, y) t(x, y)^{i},
$$

where $a_{i}(x, y) \in \mathcal{O}_{P}^{\times}$for all $i$. If $f\left(P^{\prime} \mid P\right)=1$ then by Lemma 3.10.9, we may assume that $a_{i}(x, y)=a_{i}(x)$ where $\operatorname{deg}\left(a_{i}(x)\right)<\operatorname{deg}(P)$. Taking $d$ of both sides we have that

$$
d(u(x, y))=\sum_{i=0}^{\infty}(i+1) a_{i+1}(x) t(x, y)^{i} d(t(x, y))+t(x, y)^{i} d\left(a_{i}(x)\right)
$$

Examining each term we sec that

$$
v_{P}\left(a_{i+1}(x) t(x, y)^{i} d(t(x, y))\right)=v_{P}\left(a_{i+1}(x) t(x, y)^{i}\right) \geq 0
$$

for all $i \geq 0$, and

$$
v_{P}\left(t(x, y)^{i} d\left(a_{i}(x)\right)\right)=v_{P}\left(t(x, y)^{i}\right)+v_{P}\left(d\left(a_{i}(x)\right)\right) .
$$

Now, $v_{P}\left(t(x, y)^{i}\right) \geq 0$ as $t(x, y)$ is a local parameter. In addition, by Lemma $3.10 .8, v_{P}\left(d\left(a_{i}(x)\right)\right) \geq 0$. Therefore,

$$
v_{P}\left(d(u(x, y)) \geq \min _{i \geq 0}\left\{v_{P}\left(t(x, y)^{i}\right), v_{P}\left(d\left(a_{i}(x)\right)\right)\right\} \geq 0 .\right.
$$

If $f\left(P^{\prime} \mid P\right)=2$ then one may easily check that $\{1, y\}$ furnishes a basis of $F_{P^{\prime}} / F_{P}$ and so by Lemma 3.10 .9 we may express $u(x, y)$ as a Laurent series in terms of 
$t(x, y)$ where

$$
u(x, y)=\sum_{i=0}^{\infty}\left(a_{i}(x)+b_{i}(x) y\right) t(x, y)^{i} .
$$

Taking $d$ of both sides we have that

$$
\begin{aligned}
d(u(x, y))= & \sum_{i=1}^{\infty} i\left(a_{i}(x)+b_{i}(x) y\right) t(x, y)^{i-1} d t \\
& +\sum_{i=0}^{\infty} t(x, y)^{i}\left(d\left(a_{i}(x)\right)+y d\left(b_{i}(x)\right)+b_{i}(x) d y\right) .
\end{aligned}
$$

From Lemma 3.10 .8 we know that $v_{P}\left(d\left(a_{i}(x)\right)\right) \geq 0$ and $v_{P}\left(y d\left(b_{i}(x)\right)\right) \geq 0$. Now, $d y=\frac{\varphi^{\prime}(x)}{2 y} d x$, and as we will see in Example 3.10.17, $v_{P}\left(\frac{d x}{y}\right) \geq 0$ for all places $P^{\prime} \mid P_{p(x)}$. Consequently, $v_{P}(d y) \geq 0$ for all places $P^{\prime} \mid P_{p(x)}$. Therefore,

$$
\begin{aligned}
& v_{P}(d(u(x, y))) \\
\geq & \min _{i \geq 0}\left\{v_{P}\left(\left(a_{i+1}(x)+b_{i+1}(x) y\right)\right), v_{P}\left(d\left(a_{i}(x)\right), v_{P}\left(y d\left(b_{i}(x)\right)\right), v_{P}\left(b_{i}(x) d y\right)\right\}\right. \\
\geq & 0 .
\end{aligned}
$$

2. For the case that $P^{\prime}$ lies over $P_{\infty}$, we know from Theorem 3.1.20 that $\operatorname{leg}\left(P_{\infty}\right)=$ 1 so there are two cases to consider. If $f\left(P^{\prime} \mid P_{\infty}\right)=1$ then by Lemma 3.10 .9 we may express $u(x, y)$ as

$$
u(x, y)=\sum_{i=0}^{\infty} k_{i} t(x, y)^{i}
$$

where $k_{i} \in K$, and $k_{0} \neq 0$. So taking $d$ of both sides we obtain that

$$
d(u(x, y))=\sum_{i=0}^{\infty}(i+1) k_{i+1} t(x, y)^{i} d(t(x, y))
$$

Therefore, by the Striet Triangle Inequality (Lomma 2.3.19),

$$
v_{\Gamma}(d u)=\min _{i \geq 0}\left\{(i+1) k_{i+1} t(x, y)^{i}\right\} \geq 0 .
$$


If $f\left(P^{\prime} \mid P_{\infty}\right)=2$ then from Section 3.7 we know that $\operatorname{deg}(\varphi(x))=2 m$ and one may easily check that $\left\{1, \frac{y}{x^{m}}\right\}$ furnishes a basis of $F_{P^{\prime}} / F_{P_{\infty}}$. Thus by Lemma 3.10 .9 we may express $u(x, y)$ as

$$
u(x, y)=\sum_{i=0}^{\infty}\left(a_{i}+b_{i} \frac{y}{x^{m}}\right) t(x, y)^{i}
$$

where $a_{i}, b_{i} \in K$, and one of $a_{0}, b_{0}$ is not equal to 0 . So taking $d$ of both sicles we obtain that

$$
d(u(x, y))=\sum_{i=0}^{\infty}\left((i+1) a_{i+1} t(x, y)^{i} d(t(x, y))+t(x, y)^{i} b_{i} \frac{x^{m} d y-y m x^{m-1} d x}{x^{2 m}}\right)
$$

Since $d y=\frac{\varphi^{\prime}(x) d x}{2 y}$, we see that

$$
\frac{x^{m} d y-m x^{m-1} y d x}{x^{2 m}}=\left(\frac{x \varphi^{\prime}(x)-2 m y^{2}}{2 x^{m+1}}\right) \frac{d x}{y} .
$$

First, notice that

$$
\operatorname{deg}\left(x \varphi^{\prime}(x)-2 m y^{2}\right)=\operatorname{deg}\left(x \varphi^{\prime}(x)-2 m \varphi(x)\right)<2 m
$$

since the leading coefficients cancel. Secondly, we will see in Example 3.10.17, $v_{P^{\prime}}\left(\frac{d x}{y}\right)=m-2$. Consequently,

$$
\begin{aligned}
& v_{P^{\prime}}\left(\left(\frac{x \varphi^{\prime}(x)-2 m y^{2}}{2 x^{m+1}}\right) \frac{d x}{y}\right) \\
= & \operatorname{deg}\left(2 x^{m+1}\right)-\operatorname{deg}\left(x \varphi^{\prime}(x)-2 m y^{2}\right)+v_{P^{\prime}}\left(\frac{d x}{y}\right) \\
\geq & (m+1)-(2 m-1)+(m-2) \\
= & 0 .
\end{aligned}
$$


Finally, taking the valuation of Equation (3.38) we obtain that

$$
\begin{aligned}
& v_{P^{\prime}}(d(u(x, y)) \\
= & v_{P}\left(\sum_{i=0}^{\infty}\left((i+1) a_{i+1} t(x, y)^{i} d(t(x, y))+t(x, y)^{i} b_{i} \frac{x^{m} d y-y m x^{m-1} d x}{x^{2 m}}\right)\right) \\
\geq & \min _{i \geq 0}\left\{v_{P^{\prime}}\left(a_{i+1} t(x, y)^{i} d(t(x, y))\right), v_{P^{\prime}}\left(t(x, y)^{i} \frac{b_{i}\left(x^{m} d y-y m x^{m-1}\right) d x}{x^{2 m}}\right)\right\} \\
\geq & 0 .
\end{aligned}
$$

By Theorem 3.1.20, all places $P^{\prime}$ have been considered. Therefore, $v_{P}(d u) \geq 0$ for all units $u$ in $\mathcal{O}_{P^{\prime}}$ for all $P^{\prime} \in \mathcal{P}_{F^{\prime}}$

Remark 3.10.11 Definition 3.10.7 is well-defined for $\left[F^{\prime}: K(x)\right] \leq 2$. Indeed, Let $P^{\prime}$ be a place of $F^{\prime}$ and let $t_{1} \in P^{\prime}$ be a local parameter of $P^{\prime}$. Then if $t_{2} \in P^{\prime}$ is another local parameter, then there exists a unit $u \in \mathcal{O}_{P^{\prime}}$ such that $t_{2}=u t_{1}$ (Theorem 3.1.13). Since $u$ is a unit $d u=z d t_{1}$ for some $z$ where $v_{P^{\prime}}(z) \geq 0$ (Lemma 3.10.10). As $d$ is a derivation,

$$
d t_{2}=d\left(u t_{1}\right)=u d t_{1}+t_{1} d u=u d t_{1}+t_{1} z d t_{1}=\left(u+t_{1} z\right) d t_{1} .
$$

Since $v_{P^{\prime}}(u)=0$ and $v_{P^{\prime}}\left(t_{1} z\right)>0$, by the Strict Triangle Inequality (Lemma 3.2.2), $v_{P^{\prime}}\left(u+t_{1} z\right)=0$. Let $\omega \in \Omega_{F}$. Then there exists $u_{1}$ and $u_{2}$ such that $\omega=u_{1} d t_{1}$ and $\omega=w_{2} d t_{2}$ (Proposition 3.10.6) and

$$
\begin{aligned}
w_{2} d t_{2} & =w_{1} d t_{1} \\
u_{2}\left(u-t_{1} v\right) d t_{1} & =w_{1} d t_{1} .
\end{aligned}
$$

As such, $w_{2}\left(u+t_{1} v\right)=w_{1}$. Since

$$
v_{P^{\prime}}\left(w_{1}\right)=v_{P^{\prime}}\left(u_{2}\left(u+t_{1} v\right)\right)=v_{P^{\prime}}\left(w_{2}\right)+v_{P^{\prime}}\left(u+t_{1} v\right)=v_{P^{\prime}}\left(u_{2}^{\prime}\right)
$$


the definition is well-defined.

Now that we have well defined valuation on the module of rational differential forms, we define zoros and poles of a rational differential form just as we did for elements of a function field in Definition 3.2.5.

Definition 3.10.12 Let $F$ be a function field and let $\omega \in \Omega_{F}$. We say that $\omega$ has a zero at a place $P \in \mathcal{P}_{F}$ if $v_{P}(\omega)>0$. Similarly, we way that $\omega$ has a polc at $P$ if $v_{P}(\omega)<0$.

We now wish to form a divisor of a differential form. To form a divisor, we must show that a differential form has only finitely many zeros and poles. Towards this, we first make the following observation. Since taking a valuation at a place $P$ of a differential form requires knowledge of the local parameters (Definition 3.10.7), we must first find a local parameter at each place. For $P$ in $\mathcal{P}_{K(x)}$, we have already shown this in Example 3.1.16. We have not yet done this for places of quadratic extensions of $K(x)$. We will do so now.

Example 3.10.13 Let $F=K(x)$ and $F^{\prime}=K(x, y)$ with $y^{2}=\varphi(x)$ as in Lemma 3.3.2. Using Section 3.5, we may now find local parameters at all places $P^{\prime} \in \mathcal{P}_{F^{\prime}}$. Recall from Corollary 3.2.4, that an element of $P^{\prime}$ is a local parameter if and only if it has valuation equal to 1 . Therefore, we need to find elements whose valuation is one. By Corollary 3.3.7, let $P \in \mathcal{P}_{K(x)}$ be the place lying under $P^{\prime}$, and let $t \in P$ be a local parameter at $P$. Then $v_{P^{\prime}}(t)=e \cdot v_{P}(t)=e$, so at all places $P^{\prime}$ with ramification index $e=1, t$ is a local parameter. So we really only need to consider the ramified cases, i.e. when $e>1$.

1. Let $p(x) \in K[x]$ be an irreducible polynomial. Recall from Section 3.7 that if $p(x) \mid \varphi(x)$ then there is a unique place $P^{\prime}$ lying over $P=P_{p(x)}$ which has 
relative degree $f=1$ and ramification index $e=2$. Then

$$
\begin{aligned}
v_{P}^{\prime}\left(y^{2}\right) & =v_{P}^{\prime}(\varphi(x)) \\
2 v_{P}^{\prime}(y) & =(e) v_{P}(\varphi(x)) \\
v_{P}^{\prime}(y) & =1 .
\end{aligned}
$$

So $y$ is a local parameter.

2. Recall from Section 3.7 that if $\operatorname{deg}(\varphi(x))=2 k+1$ is odd, then there is a unique place $P_{\infty}^{\prime}$ lying over $P_{\infty}$ which has relative degree $f=1$ and ramification index $e=2$. Then

$$
\begin{aligned}
v_{P}^{\prime}\left(y^{2}\right) & =v_{P}^{\prime}(\varphi(x)) \\
2 v_{P}^{\prime}(y) & =e v_{P}(\varphi(x)) \\
v_{P}^{\prime}(y) & =v_{P}(\varphi(x))=2 k+1 .
\end{aligned}
$$

Consequently,

$$
v_{P}^{\prime}\left(y x^{-k}\right)=v_{P}^{\prime}(y)+v_{P}^{\prime}\left(x^{-k}\right)=v_{P}^{\prime}(y)+2 v_{P}\left(x^{-k}\right)=2 k+1-2 k=1 .
$$

Therefore $y x^{-k}$ is a local parameter.

Proposition 3.10.14 Let $F^{\prime}=K(x, y)$ be a quadratic extention of $K(x)$ as in Lemma 3.3.2 and let $0 \neq \omega \in \Omega_{F^{\prime}}$. Then $\omega$ has finitely many zeros and poles, i.e. there exists only finitely many places $P^{\prime} \in \mathcal{P}_{F^{\prime}}$ such that $v_{P^{\prime}}(\omega) \neq 0$.

This statement is true of finite extensions of $K(x)$ in general, but we will only prove the quadratic case.

Proof. By Lemma 3.3.2. we may assime that $F^{\prime}=K(x, y)$ where $y^{2}=\varphi(x)$. From the proof of Proposition 3.10.5, $d x$ is a basis of $\Omega_{F}^{\prime}$. Hence, for all $\omega \in \Omega_{F^{\prime}}$ we have 
that $\omega=u d x$ for some $0 \neq u \in K(x, y)$. Now let $p(x)$ be an irreducible polynomial in $K[x]$ such that $p(x) \nmid \varphi(x)$. Then by Example 3.10.13, $p(x)$ is a local parameter at $P^{\prime} \mid P_{p(x)}$. Now, since $d(p(x))=p^{\prime}(x) d x$, we substitute this into our expression for $\omega$ to obtain that

$$
\omega=u d x=\frac{u}{p^{\prime}(x)} d(p(x))
$$

So taking the valuation of $\omega$ we have that

$$
\begin{aligned}
v_{P^{\prime}}(\omega) & =v_{P^{\prime}}\left(\frac{u}{p^{\prime}(x)} d(p(x))\right) \\
& =v_{P^{\prime}}(u)-v_{P^{\prime}}\left(p^{\prime}(x)\right) .
\end{aligned}
$$

Since $\operatorname{deg}(p(x))>\operatorname{deg}\left(p^{\prime}(x)\right), p(x) \nmid p^{\prime}(x)$, and thus $v_{P^{\prime}}\left(p^{\prime}(x)\right)=0$. Therefore, at all unramified places $P^{\prime}$ of $K(x, y)$, we have that

$$
v_{P^{\prime}}(\omega)=v_{P^{\prime}}(u)
$$

Since $u \in K(x, y)$, it has only finitely many zeros and poles (Theorem 3.8.7). Moreover, from Section 3.7, there are only finitely many ramified finite places of $K(x, y)$ (which correspond to the irreducible divisors of $\varphi(x)$ ). The only other possible zeros or poles are at the places lying over $P_{\infty}$ of which there are only finitely many (Lemma 3.5.1). Thercfore $\omega$ has only finitcly many zoros and poles.

Definition 3.10.15 Let $F / K(x)$ be a function field and let $P$ be a place of $F$. Let $t \in P$ be a local parameter of $P$ and let $\omega \in \Omega_{F}$. Define $\operatorname{div}_{F}(\omega)$ as the formal sum

$$
\operatorname{div}_{F}(\omega)=\sum_{P \in \mathcal{P}_{F}} v_{P}(\omega) P
$$

We call $\operatorname{div}_{F}(\omega)$ the divisor of the differential form $\omega$.

From Proposition 3.10.14, $\operatorname{div}_{F}(\omega)$ is a divisor as defined in Definition 3.8.1. In addition, as with the case of points, all divisors of $\omega \in \Omega_{F}$ are in one divisor class 
(Proposition 2.7.1); the proof is the same.

Example 3.10.16 Let $F=K(x)$. In this example we will calculate the degree of a divisor of a differential form in $\Omega_{F}$ as defined in Definition 3.8.2. Let $\omega \in \Omega_{F}$. From the proof of Proposition 3.10.5 we know that $d x$ is a generator of the module $\Omega_{F}$. Therefore $\omega=z d x$ for some $z \in F$, i.e. $\operatorname{div}(\omega) \sim \operatorname{div}(d x)$ by Definition 3.8.10. Let $p(x)$ be an irreducible polynomial in $K[x]$. Then for all finite places $P_{p(x)} \in \mathcal{P}_{F}$, we know from Example 3.1.16 that $p(x)$ is a local parameter. Since $d(p(x))=p^{\prime}(x) d x$

$$
v_{P_{p(x)}}(d x)=v_{P_{p}(x)}\left(\frac{1}{p^{\prime}(x)} d(p(x))\right)=-v_{P_{p(x)}}\left(p^{\prime}(x)\right) .
$$

However, since $\operatorname{deg}(p(x))>\operatorname{deg}\left(p^{\prime}(x)\right), p(x) \nmid p^{\prime}(x)$, and so $v_{P_{p(x)}}\left(p^{\prime}(x)\right)=0$. We have shown that $d x$ has no finite zeros or poles. To calculate the valuation at $P_{\infty}$, we know from Example 3.1.16 that $x^{-1}$ is a local parameter, and so

$$
\begin{aligned}
& d x=d\left(x^{-1} x^{2}\right) \\
& d x=x^{2} d\left(x^{-1}\right)+2 d x \\
& d x=-x^{2} d\left(x^{-1}\right) .
\end{aligned}
$$

We see that $v_{P_{\infty}}(d x)=v_{P_{\infty}}\left(-x^{2} d\left(x^{-1}\right)\right)=-2$. Therefore $\operatorname{deg}(\operatorname{div}(d x))=-2$.

Example 3.10.17 Let $F^{\prime}=K(x, y)$ be a quadratic extension of $K(x)$ as in Lemma 3.3.2. In this example we will calculate the degree of a divisor of a differential form $\omega \in \Omega_{F^{\prime}}$ as defined in Definition 3.8.2. From the proof of Proposition 3.10.5, $d x$ is a generator of $\Omega_{F^{\prime}}$. Consequently, one may easily verify that $\frac{d x}{y}$ is a generator of $\Omega_{F^{\prime}}$ as well. Therefore $\omega=z \frac{d x}{y}$ for some $z \in F^{\prime}$, i.e. $\operatorname{div}(\omega) \sim \operatorname{div}\left(\frac{1}{y} d x\right)$ by Definition 3.8.10. Let $p(x)$ be an irreducible polynomial in $K[x]$. If $p(x) \nmid \varphi(x)$ then we saw in Example 3.10.13 that $p(x)$ is a local parameter of all $P^{\prime} \mid P_{p(x)}$. Therefore, since 
$d(p(x))=p^{\prime}(x) d x$, we have that

$$
v_{P^{\prime}}\left(\frac{d x}{y}\right)=v_{P^{\prime}}\left(\frac{d(p(x))}{p^{\prime}(x) y}\right)=v_{P^{\prime}}\left(\frac{1}{p^{\prime}(x) y}\right)
$$

Since $\operatorname{deg}(p(x))>\operatorname{deg}\left(p^{\prime}(x)\right), p(x) \nmid p^{\prime}(x)$, and so $v_{P^{\prime}}\left(p^{\prime}(x)\right)=0$. From Section 3.7, since $p(x) \nmid \varphi(x)$, we know that $v_{P^{\prime}}(y)=0$ as well. Therefore $\frac{1}{y} d x$ does not have any zeros or poles at the finite unramified places of $K(x, y)$ (Section 3.7).

If $p(x) \mid \varphi(x)$ then we saw in Example 3.10.13 that $y$ is a local parameter at $P^{\prime} \mid P_{p(x)}$. Since $y^{2}=\varphi(x)$, taking the differential of both sides we see that

$$
2 y d y=\varphi^{\prime}(x) d x
$$

and so

$$
\frac{d x}{y}=\frac{2}{\varphi^{\prime}(x)} d y
$$

Since $p(x) \mid \varphi(x)$ and $\varphi(x)$ has no repeated factors $p(x) \nmid \varphi^{\prime}(x)$. Therefore, we see that

$$
v_{P^{\prime}}\left(\frac{d x}{y}\right)=v_{P^{\prime}}\left(\frac{2}{\varphi^{\prime}(x)} d y\right)=2 v_{P_{p(x)}}\left(\frac{2}{\varphi^{\prime}(x)}\right)=0 .
$$

So we have shown that $\frac{d x}{y}$ has no zeros or poles at finite ramified places of $K(x, y)$ (Section 3.7) as well. The only places we have yet to investigate are the infinite places. There are three cases to consider.

1. Suppose that the $\operatorname{deg}(\varphi(x))=n$ is odd. Then we know from Section 3.5 that there is a unique infinite place $P_{\infty}^{\prime} \in \mathcal{P}_{F^{\prime}}$. To find the value of $v_{P_{\infty}^{\prime}}\left(\frac{d x}{y}\right)$, we consider a local parameter $t \in P_{\infty}^{\prime}$. From Section 3.7, $v_{P_{\infty}^{\prime}}(x)=2$ and $v_{P_{\infty}}(y)=$ $\operatorname{deg}(\varphi(x))=n$. So by Theorem 3.1.13, we may express $x$ and $y$ as $x=t^{-2} u$ and $y=t^{-n} v$ for some units $u, v \in \mathcal{O}_{P_{\infty}^{\prime}}$. Therefore

$$
\frac{d x}{y}=\frac{-2 t^{-3} u d t+t^{-2} d u}{t^{-n} v}=\frac{-2 u d t+t d u}{v} t^{n-3} .
$$


Since $u$ is a unit $v_{P_{\infty}}(u)=0$ (Theorem 3.2.3). By Lemma 3.10.10 $v_{P_{\infty}}(d u) \geq 0$. As such, there exists a unit $h \in \mathcal{O}_{P_{\infty}^{\prime}}$ such that $d u=t^{k} h d t$ for some $k \geq 0$. This implies that

$$
\frac{d x}{y}=\frac{-2 u+t^{k+1} h}{v} t^{n-3} d(t u)
$$

Since $-2 u$ is a unit and $t^{k+1} h \in P_{\infty}, w=-2 u+t^{k+1} h$ is a unit in $\mathcal{O}_{P_{\infty}^{\prime}}$, and so is $w v^{-1}$. Therefore

$$
v_{P_{\infty}}\left(\frac{d x}{y}\right)=v_{P_{\infty}}\left(w v^{-1} t^{n-3} d t\right)=v_{P_{\infty}}\left(w v^{-1} t^{n-3}\right)=n-3
$$

So $\operatorname{deg}\left(\operatorname{div}\left(\frac{d x}{y}\right)\right)=\operatorname{deg}\left((n-3) P_{\infty}^{\prime}\right)=(n-3) f\left(P_{\infty}\right)$. From Section 3.7 we know that in this case $f\left(P_{\infty}\right)=1$ and so $\operatorname{deg}\left(\operatorname{div}\left(\frac{d x}{y}\right)\right)=n-3$.

2. Suppose that the degree of $\varphi(x)=n=2 m$ is even and the leading coefficient of $\varphi(x)$ is not a square. Then we know from Section 3.5 that there is a unique infinite place $P_{\infty}^{\prime} \in \mathcal{P}_{F^{\prime}}$ lying above $P_{\infty}$. To find the value of $v_{P_{\infty}^{\prime}}\left(\frac{d x}{y}\right)$ we again consider a local parameter $t \in P_{\infty}^{\prime}$. From Section 3.7, $v_{P_{\infty}}(x)=1$ and $v_{P_{\infty}^{\prime}}(y)=m$. So by Theorem 3.1.13, we may express $x$ and $y$ as $x=t^{-1} u$ and $y=t^{-m} v$ for some units $u, v \in \mathcal{O}_{P_{\infty}^{\prime}}$. Therefore

$$
\frac{d x}{y}=\frac{-t^{-2} u d t+t^{-1} d u}{t^{-m} v}=\frac{-u d t+t d u}{v} t^{m-2} .
$$

Since $u$ is a unit, by Theorem 3.2.3, $v_{P_{\infty}}(u)=0$, and by Lemma 3.10.10, $v_{P_{\infty}}(d u) \geq 0$. As such, there exists a unit $h \in \mathcal{O}_{P_{\infty}^{\prime}}$ such that $d u=t^{k} h d t$ for some $k \geq 0$. This implies that

$$
\frac{d x}{y}=\frac{-2 u+t^{k+1} h}{v} t^{m-2} d(t u)
$$

Since $-2 u$ is a unit and $t^{k+1} h \in P_{\infty}, w=-2 u+t^{k+1} h$ is a unit in $\mathcal{O}_{P_{\infty}^{\prime}}$ and 
thus so is $w v^{-1}$. Therefore

$$
v_{P_{\infty}}\left(\frac{d x}{y}\right)=v_{P_{\infty}}\left(w v^{-1} t^{m-2} d t\right)=v_{P_{\infty}}\left(w v^{-1} t^{m-2}\right)=m-2 .
$$

So $\operatorname{deg}\left(\operatorname{div}\left(\frac{d x}{y}\right)\right)=\operatorname{deg}\left((m-2) P_{\infty}^{\prime}\right)=(m-2) f\left(P_{\infty}\right)$. From Section 3.7 we know that in this case $f\left(P_{\infty}\right)=2$. Therefore $\operatorname{deg}\left(\operatorname{div}\left(\frac{d x}{y}\right)\right)=(m-2)(2)=n-4$.

3. Suppose that the degree of $\varphi(x)=n=2 m$ is even and the leading coefficient of $\varphi(x)$ is a square. Then we know from Section 3.5 that there are exactly two infinite places lying over $P_{\infty}$. Let $P_{\infty, 1}^{\prime}$ be one of the places lying over $P_{\infty}$. To find the value of $v_{P_{\infty, 1}^{\prime}}\left(\frac{d x}{y}\right)$ we again consider a local parameter $t \in P_{\infty, 1}^{\prime}$. From Section $3.7 v_{P_{\infty, 1}^{\prime}}(x)=1$ and $v_{P_{\infty, 1}^{\prime}}(y)=m$. So by Theorem 3.1.13, we may express $x$ and $y$ as $x=t^{-1} u$ and $y=t^{-m} v$ for some units $u, v \in \mathcal{O}_{P_{\infty, 1}^{\prime}}$. Following the calculations of the previous case we obtain that

$$
v_{P_{\infty, 1}^{\prime}}\left(\frac{d x}{y}\right)=v_{P_{\infty, 1}^{\prime}}\left(w v^{-1} t^{m-2} d t\right)=m-2
$$

Let $P_{\infty, 2}^{\prime}$ be the other place lying over $P_{\infty}$. To find the value of $v_{P_{\infty, 2}^{\prime}}\left(\frac{d x}{y}\right)$ we again consider a local parameter $t$ at $P_{\infty, 2}^{\prime}$. From Section $3.7 v_{P_{\infty}^{\prime}}(x)=1$ and $v_{P_{\infty}^{\prime}}(y)=m$. So again, we obtain that

$$
v_{P_{\infty}^{\prime}}\left(\frac{d x}{y}\right)=m-2
$$

Finally,

$$
\operatorname{deg}(\operatorname{div}(d x / y))=\operatorname{deg}\left((m-2) P_{\infty, 1}^{\prime}+(m-2) P_{\infty, 1}^{\prime}\right)=(m-2)+(m-2)=n-4
$$

Definition 3.10.18 We say that a differential form $\omega \in \Omega_{F}$ is regular if $\operatorname{div}_{F}(\omega) \geq 0$.

Just as in the case of divisors of points (Section 2.5), we may define $L(D)$, the space of a divisor $D$ of places (Defintion 2.7.3). It follows again that $L(D)$ is a vector 
space over $K$ of dimension less than or equal to $\operatorname{deg}(D)+1$. It also follows that divisors in the same divisor class have isomorphic divisor spaces. So just as we did for curves (Definition 2.7.12), we may define the genus of a function field as follows.

Definition 3.10.19 Let $F / K(x)$ be a function field and let $\omega \in \Omega_{F}$ be a rational differential form. The genus of the function field $F$, denoted $g_{F}$, is defined as

$$
g_{F}=\operatorname{dim}_{K}\left(L\left(\operatorname{div}_{F}(\omega)\right)\right)
$$

Remark 3.10.20 The vector space $L\left(\operatorname{div}_{F}(\omega)\right)$ is again isomorphic to the space of regular differential forms. Also recall from Definition 2.7.3, that $L\left(\operatorname{div}_{F}(\omega)\right)$ always contains the zero function. Moreover, from Definition 2.7.3, if $\operatorname{deg}(\operatorname{div}(\omega))<0$ then $L\left(\operatorname{div}_{F}(\omega)\right)=\{0\}$. From Example 3.10.16, we know that $\operatorname{deg}(\operatorname{div}(\omega))=-2$ for all $\omega \in \Omega_{K(x)}$. Therefore, $K(x)$ has genus $g_{K(x)}=0$.

Example 3.10.21 Continuing from Example 3.10.17, let $\omega=\frac{d x}{y}$. We look at the space of regular differential forms over $K$ by examining $L(\operatorname{div}(\omega))$. By the definition of $L(\operatorname{div}(\omega))$, Definition 2.7.3, any element of $L(\operatorname{div}(\omega))$ is an $h \in F^{\prime}$, such that

1. $h$ has no finite poles since $\omega$ has no finite zeros (Example 3.10.17). Hence $h$ must be of the form $a(x)+b(x) y$ with $a(x), b(x) \in K[x]$.

2. $h$ must satisfy

$$
v_{P^{\prime}}(h) \geq-v_{P^{\prime}}(\omega) \text { for all } P^{\prime} \mid P_{\infty}
$$

Let $P_{\infty}^{\prime}$ be a place in $K(x, y)$ such that $P^{\prime} \mid P_{\infty}$. We know from the definition of a valuation that

$$
v_{P_{\infty}^{\prime}}(h)=v_{P_{\infty}^{\prime}}(a(x)+b(x) y) \leq \min \left\{v_{P_{\infty}^{\prime}}(a(x)), v_{P_{\infty}^{\prime}}(b(x) y)\right\} .
$$


Suppose that $b(x) \neq 0$. Then

$$
\begin{aligned}
v_{P_{\infty}^{\prime}}(h) & \leq v_{P_{\infty}^{\prime}}(b(x) y) \\
& =v_{P_{\infty}^{\prime}}(b(x))+v_{P_{\infty}^{\prime}}(y) \\
& \leq v_{P_{\infty}^{\prime}}(y) \\
& \leq-v_{P_{\infty}^{\prime}}\left(y^{-1}\right)
\end{aligned}
$$

Using that $v_{P_{\infty}}(d x)=-2$ (Example 3.10 .16$)$, we obtain that

$$
\begin{aligned}
v_{P_{\infty}^{\prime}}(h) & <-v_{P_{\infty}^{\prime}}\left(y^{-1}\right)-e\left(P_{\infty}^{\prime} \mid P_{\infty}\right) v_{P_{\infty}}(d x) \\
& =-\left(v_{P_{\infty}^{\prime}}\left(y^{-1}\right)-v_{P_{\infty}^{\prime}}(d x)\right) \\
& =-\left(v_{P_{\infty}^{\prime}}\left(y^{-1} d x\right)\right) \\
& =-v_{P_{\infty}^{\prime}}(\omega)
\end{aligned}
$$

However, this contradicts equation (3.39) and thus $b(x)=0$. Now, examining the remaining possibilities for $h$ we see that $h=a(x)$ where

$$
v_{P_{\infty}^{\prime}}(a(x)) \geq-v_{P_{\infty}^{\prime}}(\omega)
$$

Let's again break this down into the three cases of Example 3.10.17.

1. If the $\operatorname{deg}(\varphi(x))=n$ is odd then equation (3.40) implies that

$$
\begin{aligned}
v_{P_{\infty}^{\prime}}(a(x)) & \geq-v_{P_{\infty}^{\prime}}(\omega) \\
2 v_{P_{\infty}}(a(x)) & \geq-(n-3) \\
-2 \operatorname{deg}(a(x)) & \geq-(n-3) \\
2 \operatorname{deg}(a(x)) & \leq(n-3) .
\end{aligned}
$$

Since $n$ is odd, let $n=2 k+1$. Thus, we see that $\operatorname{deg}(a(x)) \leq k-1$. 
2. If the degree of $\varphi(x)=n=2 m$ is even and the leading coefficient of $\varphi(x)$ is not square then equation (3.40) implies that

$$
\begin{aligned}
v_{P_{\infty}^{\prime}}(a(x)) & \geq-v_{P_{\infty}^{\prime}}(\omega) \\
v_{P_{\infty}}(a(x)) & \geq-(m-2) \\
-\operatorname{deg}(a(x)) & \geq-(m-2) \\
\operatorname{deg}(a(x)) & \leq(m-2) .
\end{aligned}
$$

Since $2 m$ is even, let $2 m=2 k+2$. Then we obtain that $\operatorname{deg}(a(x)) \leq k-1$.

3. If the degree of $\varphi(x)=2 m$ is even and the leading coefficient of $\varphi(x)$ is a square then for any $P^{\prime} \mid P_{\infty}$ equation (3.40) implies that

$$
\begin{aligned}
v_{P^{\prime}}(a(x)) & \geq-v_{P_{\infty}^{\prime}}(\omega) \\
v_{P_{\infty}}(a(x)) & \geq-(m-2) \\
-\operatorname{deg}(a(x)) & \geq-(m-2) \\
\operatorname{deg}(a(x)) & \leq(m-2) .
\end{aligned}
$$

Since $2 m$ is even, let $2 m=2 k+2$. From above, we see that $\operatorname{deg}(a(x)) \leq k-1$.

In conclusion, the dimension of the vector space of regular differential forms, i.e the genus, of the function field $K(x, y)$ is $g=g_{K(x, y)}=k$ where $\operatorname{deg}(\varphi(x))=2 g+1$ or $\operatorname{deg}(\varphi(x))=2 g+2$.

As we have just seen, there is a strong relation between the degree of the divisor of a differential form and the genus of the function field.

Theorem 3.10.22 Lct $F / K(x)$ be a function field and let $\omega \in \Omega_{F}$. Then

$$
\left.\operatorname{deg}\left(\operatorname{div}_{F}(\omega)\right)\right)=2 g_{F}-2
$$


This is a direct consequence of the Riemann-Roch Theorem, which we will not prove in general. For a proof see ([19] p.28). We will prove Theorem 3.10 .22 for field extensions of degree less than or equal to two.

Proof. If $F$ equals $K(x)$ then in Example 3.10 .16 we saw that $\left.\operatorname{deg}\left(\operatorname{div}_{F}(\omega)\right)\right)=$ -2 . As a result, in Remark 3.10.20 we showed that the genus is 0 and thus the desired equality holds. For the case of $F=K(x, y)$ as in Lemma 3.3.2, let $n=\operatorname{deg}(\varphi(x))$. Then we have seen in Example 3.10.17 that if $\operatorname{deg}(\varphi(x))$ is odd then $\left.\operatorname{deg}\left(d i v_{F}(\omega)\right)\right)=$ $n-3$ and if it is even then $\left.\operatorname{deg}\left(\operatorname{div}_{F}(\omega)\right)\right)=n-4$. Moreover, in Example 3.10.21, we saw that if $\operatorname{deg}(\varphi(x))$ is odd then $n=2 g_{F}+1$ and if it is even then $n=2 g+2$. Therefore, in both cases, substituting in for $n$ we obtain that

$$
\left.\operatorname{deg}\left(\operatorname{div}_{F}(\omega)\right)\right)=2 g_{F}-2
$$

\subsection{The Different and The Riemann Hurwitz Genus Formula}

At this point, we may calculate the genus of a function field $F^{\prime}$ which is quadratic extension of $K(x)$ (Theorem 3.10.22). The aim of this section is the converse. Given the genus of $F^{\prime}$, and the fact that it is quadratic, we determine the possibilities for $F^{\prime}$.

Definition 3.11.1 ([5] p.76) Let $F^{\prime}$ be a finite extension of a function field $F$ and let $0 \neq \omega \in \Omega_{F}$. Let $P \in \mathcal{P}_{F}$ and let $P^{\prime} \in \mathcal{P}_{F^{\prime}}$ be such that $P^{\prime} \mid P$. Then the differential exponent is defined as

$$
d\left(P^{\prime} \mid P\right):=v_{P^{\prime}}(\omega)-e\left(P^{\prime} \mid P\right) v_{P}(\omega)
$$

We prove that this definition is independent of the choice of $\omega$. Let $t \in P$ be a local parameter at $P$. Then for any $\omega \in \Omega_{F}$ there exists $u \in F$ such that $\omega=u d t$. by 
Proposition 3.10.6. Defining $e:=e\left(P^{\prime} \mid P\right)$ we see that

$$
\begin{aligned}
d\left(P^{\prime} \mid P\right) & =v_{P^{\prime}}(\omega)-e v_{P}(\omega) \\
& =v_{P^{\prime}}(u d t)-e v_{P}(u d t) \\
& =v_{P^{\prime}}(u)+v_{P^{\prime}}(d t)-e\left(v_{P}(u)+v_{P}(d t)\right) \\
& =e v_{P}(u)+v_{P^{\prime}}(d t)-e v_{P}(u)-e v_{P}(d t) .
\end{aligned}
$$

Since $v_{P}(u)=0$

$$
d\left(P^{\prime} \mid P\right)=v_{P^{\prime}}(d t)-e v_{P}(d t)
$$

It follows from Equation (3.41) and Proposition 3.10.6 that indeed the definition of $d\left(P^{\prime} \mid P\right)$ is independent of the choice of $\omega \in \Omega_{F}$. From Definition 3.11.1, we also see that $d\left(P^{\prime} \mid P\right)$ is the coefficient of $P^{\prime}$ in the divisor $\operatorname{div}(\omega)_{F^{\prime}}-\operatorname{Con}_{F^{\prime} / F}\left(\operatorname{div}(\omega)_{F}\right)$ as defined in Definition 3.9.1. In particular, this means that $d\left(P^{\prime} \mid P\right)$ is zero at all but finitely many $P^{\prime} \in \mathcal{P}_{F^{\prime}}$. This allows us to construct a divisor based upon the differential exponents.

Definition 3.11.2 Let $F^{\prime}$ be a finite extension of a function field $F$ and let $\omega \in \Omega_{F}$ be non-zero. Let $P \in \mathcal{P}_{F}$ and let $P^{\prime} \in \mathcal{P}_{F^{\prime}}$ be such that $P^{\prime} \mid P$. Define the different of the extension, which we denote as $D i f f_{F^{\prime} / F}$, to be

$$
\operatorname{Diff}_{F^{\prime} / F}:=\sum_{P^{\prime} \in \mathcal{P}_{F^{\prime}}} d\left(P^{\prime} \mid P\right) P^{\prime}
$$

As per the comments before the definition,

$$
\operatorname{Diff}_{F^{\prime} / F}=\operatorname{div}(\omega)_{F^{\prime}}-\operatorname{Con}_{F^{\prime} / F}\left(\operatorname{div}(\omega)_{F}\right) .
$$

Theorem 3.11.3 ([5] p.77) Let $F^{\prime}$ be a finite extension of a function field $F$. Let $P \in \mathcal{P}_{F}$ and let $P^{\prime} \in \mathcal{P}_{F^{\prime}}$ be such that $P^{\prime} \mid P$. Then $d\left(P^{\prime} \mid P\right)=e\left(P^{\prime} \mid P\right)-1$, unless $\operatorname{char}(K) \mid e\left(P^{\prime} \mid P\right)$, in which case $d\left(P^{\prime} \mid P\right) \geq e\left(P^{\prime} \mid P\right)$. 
Proof. Let $s, t$ be local parameters at $P^{\prime}$ and $P$ respectively, i.e. $v_{P^{\prime}}(s)=v_{P}(t)=1$.

Then by Theorem 3.1.13, $t=u s^{e}$ for some $u$ is a unit in $\mathcal{O}_{P^{\prime}}$ and $e:=e\left(P^{\prime} \mid P\right)$. From equation (3.41) we have that

$$
\begin{aligned}
d\left(P^{\prime} \mid P\right) & =v_{P^{\prime}}(d t)-e v_{P}(d t) \\
& =v_{P^{\prime}}(d t)-e v_{P}(1) \\
& =v_{P^{\prime}}\left(d\left(s^{e} u\right)\right)-0 \\
& =v_{P^{\prime}}\left(e s^{e-1} u d s+s^{e} d u\right) \\
& =v_{P^{\prime}}\left(s^{e-1}(e u d s+s d u)\right) .
\end{aligned}
$$

Since $u$ is a unit, by Lemma 3.10.10, $d u=w d s$ where $v_{P^{\prime}}(w) \geq 0$. Now we see that

$$
\begin{aligned}
& d\left(P^{\prime} \mid P\right)=v_{P^{\prime}}\left(s^{e-1}(e u d s+s d u)\right) \\
& d\left(P^{\prime} \mid P\right)=v_{P^{\prime}}\left(s^{e-1}(e u+s w) d s\right) .
\end{aligned}
$$

If $\operatorname{char}(k) \nmid e$ then $e u$ is a unit in $\mathcal{O}_{P}$ and $s w \in P$. Therefore $e u+s w$ is a unit in $\mathcal{O}_{P}$ and

$$
d\left(P^{\prime} \mid P\right)=v_{P^{\prime}}\left(s^{e-1}(e u+s w) d s\right)=e-1 .
$$

Alternatively, if $\operatorname{char}(K) \mid e$, then

$$
d\left(P^{\prime} \mid P\right)=v_{P^{\prime}}\left(s^{e-1}(e u+s w) d s\right)=v_{P^{\prime}}\left(s^{e} u d s\right)>e .
$$

Example 3.11.4 Let $F^{\prime}=K(x, y)$ be a quadratic extension of $F=K(x)$ where $y^{2}=\varphi(x)$ as in Lemma 3.3.2. Then we may decompose $\varphi(x)$ as

$$
\varphi(x)=\prod_{i=1}^{m} p_{i}(x) \text { where } p_{i}(x) \in K[x] \text { is irreducible for all } i=1, \ldots, m
$$


Set $P_{p_{i}}=P_{p_{i}(x)}$. We have shown in Section 3.7 that only ramified places of $F^{\prime}$ are the unique places $P_{p_{i}}^{\prime} \in \mathcal{P}_{F^{\prime}}$ such that $P_{p_{i}}^{\prime} \mid P_{p_{i}}$, except when $\operatorname{deg}(\varphi)=n$ is odd, in which case $P_{\infty}^{\prime}$ is ramified as well. Thus, we have that

$$
\operatorname{Diff}_{F^{\prime} / F}=\left\{\begin{array}{ll}
\sum_{i=1}^{n} P_{i}^{\prime} & \text { if } \operatorname{deg}(\varphi)=n \text { is even } \\
P_{\infty}^{\prime}+\sum_{i=1}^{n} P_{i}^{\prime} & \text { if } \operatorname{deg}(\varphi)=n \text { is odd }
\end{array} .\right.
$$

Perhaps of more importance is the degree of the divisor $\operatorname{Diff}_{F^{\prime} / F}$.

$$
\operatorname{deg}\left(\operatorname{Diff}_{F^{\prime} / F}\right)=\left\{\begin{array}{ll}
\operatorname{deg}(\varphi(x)) & \text { if } \operatorname{deg}(\varphi(x)) \text { is even } \\
\operatorname{deg}(\varphi(x))+1 & \text { if } \operatorname{deg}(\varphi(x)) \text { is odd }
\end{array} .\right.
$$

Theorem 3.11.5 (Riemann Hurwitz Genus Formula) ([5] p.76) Let $F^{\prime}$ be a finite extension of a function field $F$. Let $g$ denote the genus of $F$ and $g^{\prime}$ denote the genus of $F^{\prime}$. We have

$$
2 g^{\prime}-2=\left[F^{\prime}: F\right](2 g-2)+\operatorname{deg}\left(D_{i f f} F_{F^{\prime} / F}\right) .
$$

Proof. By Definition 3.11.2 we have that,

$$
\operatorname{Diff} F_{F^{\prime} / F}=\operatorname{div}(\omega)_{F^{\prime}}-C_{o n} n_{F^{\prime} / F}\left(\operatorname{div}(\omega)_{F}\right) .
$$

Using Theorem 3.10 .22 and checking the degree of both sides we see that

$$
\operatorname{deg}\left(\operatorname{Diff}_{F^{\prime} / F}\right)=\left(2 g^{\prime}-2\right)-\operatorname{deg}\left(\operatorname{Con}_{F^{\prime} / F}\left(\operatorname{div}(\omega)_{F}\right)\right)
$$

From Proposition 3.9.3 we have that

$$
\begin{aligned}
\operatorname{deg}\left(D_{i f f_{F^{\prime} / F}}\right) & \left.=\left(2 g^{\prime}-2\right)-\left[F^{\prime}: F\right] \operatorname{deg}\left(\operatorname{div}(\omega)_{F}\right)\right) \\
\operatorname{deg}\left(D_{i f f_{F^{\prime} / F}}\right) & =\left(2 g^{\prime}-2\right)-\left[F^{\prime}: F\right](2 g-2) \\
\left(2 g^{\prime}-2\right) & =\left[F^{\prime}: F\right](2 g-2)-\operatorname{deg}\left(\operatorname{Diff}_{F^{\prime} / F}\right)
\end{aligned}
$$


as desired.

Since we have explicitly proven Theorem 3.10 .22 in the case of quadratic extensions, we have proven the Riemann Hurwitz genus formula for these extensions as well. We will state this result in the following corollary.

Corollary 3.11.6 Let $F^{\prime}$ be a quadratic extension of $K(x)$. Let $g_{F^{\prime}}$ denote the genus of $F^{\prime}$ Then we have

$$
2 g_{F^{\prime}}+2=\operatorname{deg}\left(D i f f_{F^{\prime} / F}\right)
$$

Proof. By Remark 3.10.20, $g_{K(x)}=0$. So substituting $g=0$ and $\left[F^{\prime}: F\right]=2$ into the Riemann Hurwitz genus formula gives this result.

Theorem 3.11.7 Let $F^{\prime}$ be a quadratic extension of $K(x)$ of genus $g$. Then $F^{\prime}=$ $K(x, y)$ for some element $y$ such that $y^{2}=\varphi(x)$ where $\varphi(x) \in K[x]$ has degree $2 g+1$ or $2 g+2$.

Proof. Since $F^{\prime}$ be a quadratic extension of $K(x)$, by Lemma 3.3.2, $F^{\prime}=K(x, y)$ for some clement $y$ such that $y^{2}=\varphi(x)$ where $\varphi(x) \in K[x]$. By Corollary 3.11.6,

$$
\operatorname{deg}\left(\operatorname{Diff}_{F^{\prime} / F}\right)=2 g+2
$$

Moreover, from Example 3.11.4,

$$
\operatorname{deg}(\varphi(x))=\operatorname{deg}\left(D \text { iff }_{F^{\prime} / F}\right) \quad \text { or } \quad \operatorname{deg}(\varphi(x))=\operatorname{deg}\left(D \text { iff }_{F^{\prime} / F}\right)-1
$$

Combining theso equations we attain that

$$
\operatorname{deg}(\varphi(x))=2 g+2 \quad \text { or } \quad \operatorname{deg}(\varphi(x))=2 g+1
$$

as required. 


\section{Chapter 4}

\section{Algebra and Geometry}

We again return to the case that $K$ algebraically closed with characteristic different than 2. In this chapter, we will relate our work on function fields back to curves. We will determine the relationship between the points on a nonsingular curve and the places of a function field. We are finally able to define and explicitly describe a hyperelliptic curve. We will prove that the genus of a hyperelliptic curve is equal to the genus of its function field. Lastly, we will show that all hyperelliptic curves are birationally equivalent to the projective closures of the affine curves defined by the equation $y^{2}=\varphi(x)$.

\subsection{Hyperelliptic Curves}

We begin by restating the definition of a hyperelliptic curve from the introduction.

Definition 4.1.1 ([1] p.73) An irreducible nonsingular projective curve $C$ of genus $g \geq 1$ is called a hyperelliptic curve if the function field $K(C)$ is a degree two extension of the rational function field $K(x)$.

Hyperelliptic curves with genus $g=1$ are called elliptic curves. Following usual convention, we include elliptic curves as a subset of hyperelliptic curves. From the 
previous chapters we may give a much more explicit description of a hyperelliptic curve.

Lemma 4.1.2 ([4] p.161) There is a one-to-one correspondence between the points of hyperelliptic curve $C$ and the discrete valuations of the function ficld $K(C)$.

This is true in general for nonsingular projective curves $C$ ([4] p.161), but we will only prove the case where $C$ is hyperelliptic.

Proof. By Lemma 3.3.2 we may assume without loss of generality that $K(C)$ is isomorphic to $K(x, y)$ where $y^{2}=\varphi(x)$. In addition, since $K$ is algebraically closed, we may assume that the degree of $\varphi(x)$ is odd (Example 2.1.62). By Theorem 2.3.9, the points of $C$ correspond injectively to discrete valuation rings. Thus, by Definition 2.3.2, $\left\{M_{p}\right\} \subset \mathcal{P}_{K(C)}$. Since $K$ is algebraically closed, all irreducible polynomials $p(x) \in K[x]$ are linear. Therefore, each place $P \in K(x)$ is either of the form $P_{x-a}$ for some $a \in K$ or $P_{\infty}$. In addition, we saw in Section 3.5 that $f\left(P^{\prime} \mid P\right)=2$ if and only if the remainder of $\varphi(x) /(x-a)$ is not a square in $K$. The algebraic closure of $K$ guarantees that every element of $K$ is a square and thus $f\left(P^{\prime} \mid P\right)=1$ in all cases. Consequently, there are only two cases to consider.

If $(x-a) \mid \varphi(x)$ then $a$ is root of $\varphi(x)$ then we saw in Section 3.5 that there was a unique place $P^{\prime} \mid P$. This place corresponds to the ideal $M_{(a, 0)}$. If $(x-a) \nmid \varphi(x)$ then there are two distinct places $P_{1}^{\prime} \mid P$ and $P_{2}^{\prime} \mid P$. Let $b \in K$ be such that $b^{2}-\varphi(a)=0$ then places $P_{1}^{\prime}$ and $P_{2}^{\prime}$ correspond to the ideals $M_{(a, b)}$. Lastly, there exists at least one point on $C$ which is birationally mapped to the infinite point. However, as we saw in Section 3.5 , there is only one more place of $K(x, y)$; the unique place lying over $P_{\infty}$. Therefore, $C$ has a unique infinite point $p_{\infty}$ and its ideal corresponds to the ideal $P_{\infty}^{\prime}$. Since all places are accounted for, $\left\{M_{p}\right\}=\mathcal{P}_{K(C)}$.

Let $C$ be the projective closure of the affine plane curve defined by $y^{2}-\varphi(x)$. Observe from the proof of Lemma 4.1.2, that there is a unique point of the nonsingular projective model $C^{\prime}$ of $C$ corresponding to the point $p_{\infty}$. This justifies the claim we made in Scction 2.4. 
Theorem 4.1.3 Let $C$ be a hyperelliptic curve. Then the genus of the curve $g_{C}$ is equal to the genus of the function field $g_{K(C)}$.

Proof. Recall from Definition 3.10.19 that $g_{K(C)}=\operatorname{dim}_{K}(L(\operatorname{div}(\omega)))$ for some $\omega \in$ $\Omega_{K\left(C^{\prime}\right)}$. Also recall from Definition 2.7.12 that $g_{C}=\operatorname{dim}_{K}\left(L\left(\operatorname{div}\left(\omega_{C}\right)\right)\right)$ for some $\omega_{C} \in \Omega(C)$. We will show that $\Omega(C) \cong \Omega_{K(C)}$ and the result will follow.

Let $F=K(C)$ and let $\omega_{F} \in \Omega_{F}$. Define the linear map $\phi: \Omega_{K(C)} \rightarrow \Omega(C)$ where by $\phi\left(\omega_{F}\right)$ is the equivalence class of $\left(\omega_{F}, U\right)$, where $U$ is the open set of points of $C$ where $\omega_{F}$ is defined, i.e. $C$ minus the finite set of poles of $\omega_{F}$ (Proposition 3.10.14). We claim that $\phi$ is an isomorphism. Indeed, for all $\left(\omega_{C}, U\right), \omega_{C}$ can be written as

$$
\omega_{C}=\sum_{i=1}^{m} h_{i}(t) d\left(g_{i}(t)\right)
$$

for some $h_{i}(t), g_{i}(t) \in K[U]$ for all $i=1, \ldots, m$ (Equation (2.14)). As $h_{i}(t), g_{i}(t)$ are regular on an open subset of $C$ they are rational on $C$ (Proposition 2.1.55). Thus, there exists an elcment $\omega_{F}=\sum_{i=1}^{m} h_{i}(t) d\left(g_{i}(t)\right) \in K(C)$ such that $\phi\left(\omega_{F}\right)=$ $\left(\omega_{F}, U_{1}\right) \sim\left(\omega_{C}, U\right)$. Therefore, $\phi$ is surjective.

Suppose that $\phi\left(\omega_{1}\right)=\phi\left(\omega_{2}\right)$ for some $\omega_{1}, \omega_{2} \in \Omega_{K(C)}$. This implies that $\left(\omega_{1}, U_{1}\right) \sim$ $\left(\omega_{2}, U_{2}\right)$. By Proposition 2.6.20 this is means that $\omega_{1}=\omega_{2}$ on $U_{1} \cap U_{2}$. Let $s$ be a local parameter at a nonsingular point $p \in U$ then by Corollary 2.6.15, $\omega_{1}=g_{1} d s$ and $\omega_{2}=g_{2} d s$ on $U_{1} \cap U_{2}$ for some $g_{1}, g_{2} \in K[U]$. Consequently,

$$
\begin{aligned}
\omega_{1} & =\omega_{2} \text { on } U_{1} \cap U_{2} \\
g_{1} d s & =g_{2} d s \text { on } U_{1} \cap U_{2} \\
g_{1} & =g_{2} \text { on } U_{1} \cap U_{2} .
\end{aligned}
$$

As $g_{1}=g_{2}$ on an open subset of $C$, Proposition 2.1.55 tells us that $g_{1}=g_{2} \epsilon$ $K(C)$. Consequently, $\omega_{1}=\omega_{2}$ and thus $\phi$ is injective. Therefore $\Omega(C) \cong \Omega_{K(C)}$. By Lemma 4.1.2, there is a bijection between the points of $C$ and the places $P \in \mathcal{P}_{K(C)}$. 
Consequently, there is a bijection $\operatorname{div}_{F}\left(\omega_{F}\right) \leftrightarrow d i v_{C}\left(\omega_{C}\right)$. As $K(C) \cong K(x, y)$ (Lemma 3.3.2), we obtain that

$$
g_{C}=\operatorname{dim}_{K}\left(L\left(\operatorname{div}\left(\phi\left(\omega_{F}\right)\right)\right)\right)=\operatorname{dim}_{K}\left(L\left(\operatorname{div}\left(\omega_{F}\right)\right)\right)=g_{K(C)}
$$

Theorem 4.1.4 A curve $C$ of genus $g \geq 1$ is hyperelliptic if and only if it is a nonsingular projective model of the projective closure of an affine curve defined by $y^{2}-\varphi(x)$, where $\varphi(x)$ is squarefree and deg $g(\varphi(x))=2 g+1$.

Proof. One direction is clear. Let $C$ be an irreducible nonsingular projective model of the projective closure $D$ of the affine curve defined by $y^{2}-\varphi(x)$ where $\varphi(x)$ is square free and $\operatorname{deg}(\varphi(x))=2 g+1 . K(D)$ is quadratic as per Remark 2.1.52. Theorem 2.1.75 implies that $K(D) \cong K(C)$, and so $K(C)$ is quadratic as well. From Example 2.7.14, the genus of $C$ and $D$ are both equal to $g$. Thus $C$ is a hyperelliptic curve by Definition 4.1.1.

Conversely, Let $C$ be a hyperelliptic curve. By Definition 4.1.1, $K(C)$ is a quadratic extension of $K(x)$. By Lemma 3.3.2, $K(C) \cong K(x, y)$ where $y^{2}=\varphi(x)$, $\varphi(x)$ has no repeated roots and $\operatorname{deg}(\varphi(x))$ is odd (Example 2.1.62). By Theorem 4.1.3, the genus of $K(x, y)$ is also $g$. Therefore, by Theorem 3.11.7, we know that the degree of $\varphi(x)=2 g+1$. Let $D$ be the projective closure of the affine curve defined by the equation $y^{2}-\varphi(x)$. Then $K(D) \cong K(x, y) \cong K(C)$. Therefore, by Theorem 2.1.75, $D$ is birationally equivalent to $C$. As $C$ is nonsingular, it is a nonsingular projective model of $D$. 


\section{Chapter 5}

\section{Conclusions}

After examining the definition of a hyperelliptic curve, as given in Definition 1.1, we were able to present a more tangible description of a hyperelliptic curve in Theorem 4.1.4; one in terms of $y^{2}-\varphi(x)$. In Section 2.4, we also showed how it is nearly sufficient to restrict our attention to the curve defined by this equation. This is why hyperelliptic curves are so often treated at curves defined by $y^{2}-\varphi(x)$ together with a "point at infinity".

In persuit of this endeavor we were in fact able to accomplish much more. For instance, in Chapter 3 we were able to completely describe all the places of a hyperelliptic function field over an arbitrary field $K$, the only restriction being that $\operatorname{char}(K) \neq 2$. This is especially important as most literature on the subject does not provide such a complete account. They either restrict themselves to algebraically closed fields $K$ or express these places in term of the algebraic closure of $K$.

One should also look into the case that $\operatorname{char}(K)=2$. In this case there are plenty of interesting things that can happen. For instance, we cannot complete the square to assume that $y^{2}=\varphi(x)$; we must consider curves of the form $y^{2}-h(x) y-f(x)=0$. Secondly, we can have wild ramification, i.e. $d\left(P^{\prime} \mid P\right)$ can be greater than 1. As such, the genus is less strongly related to the degree of $h$ and $f$. Sec [19] and [6] for examples. 
For those interested in studying hyperelliptic curves further, there are plenty of resources available. Fulton [4] provides a good introduction to the algebraic geometry of curves. A more advanced book would be Shafarevich [16]. It is rather terse and lacks a lot of structure but it contains plenty of good information. For those interested in advanced topics in algebraic geometry, including things like sheaves and schemes, Hartshorne [6] is recommended. Finally, for those interested in hyperelliptic function ficlds, Stichtenoth [19] is a solid introduction; it does however lack examples.

Given that hyperelliptic curves are so utilized in computer algorithms, it would be a great injustice not to mention that area of interest. Koblitz [9] serves as a good introduction to elliptic and hyperelliptic curve based cryptography. His book provides a great description of the group structure associated to a hyperelliptic curve. Those wishing to persue the subject in detail must consult the handbook [1] for further direction.

There are other specific areas of interest that we have not yet covered. Perhaps the most interesting is the ring theoretic version of the group structure of a hyperelliptic curve. One can form a group on the maximal ideals of the ring $K[x, y]$ where $y^{2}=$ $\varphi(x)$. In the case thiat $K$ is algebraically closed, this group is formed using the ideals $(x-a, y-b)$, where $b^{2}=\varphi(a)$; just as in the case of places. However, when $K$ is not algebraically closed, one wonders what these ideals become. One would assume that these ideals become the ring theoretic version of the places of $K(x, y)$. This would of course require a proof and further investigation. 


\section{Bibliography}

[1] Henri Cohen, Gerhard Frey, Roberto Avanzi, Christophe Doche, Tanja Lange, Kim Nguyen, and Frederik Vercauteren (eds.), Handbook of elliptic and hyperelliptic curve cryptography, Discrete Mathematics and its Applications (Boca Raton), Chapman \& Hall/CRC, Boca Raton, FL, 2006.

[2] David S. Dummit and Richard M. Foote, Abstract algebra, third ed., John Wiley \& Sons Inc., Hoboken, NJ, 2004.

[3] Irwin Fischer, The moduli of hyperelliptic curves, Trans. Amer. Math. Soc. 82 (1956), 64-84.

[4] William Fulton, Algebraic curves. An introduction to algebraic geometry, W. A. Benjamin, Inc., New York-Ansterdam, 1969, Notes written with the collaboration of Richard Weiss, Mathematics Lecture Notes Series.

[5] David M. Goldschmidt, Algebraic functions and projective curves, Graduate Texts in Mathematics, vol. 215, Springer-Verlag, New York, 2003.

[6] Robin Hartshorne, Algebraic geometry, Springer-Verlag, New York, 1977, Graduate Texts in Mathematics, No. 52.

[7] Nathan Jacobson, Basic algebra. II, second ed., W. H. Freeman and Company, New York, 1989.

[8] Neal Koblitz, Hyperelliptic cryptosystems, J. Cryptology 1 (1989), no. 3, 139150 .

[9] _ Algebraic aspects of cryptography, Algorithms and Computation in Mathematics, vol. 3, Springer-Verlag, Berlin, 1998, With an appendix by Alfred J. Menezes, Yi-Hong Wu and Robert J. Zuccherato.

[10] Tanja Lange and Igor E. Shparlinski, Certain exponchtial sums and random walks on elliptic curves, Canad. J. Math. 57 (2005), no. 2, 338-350.

[11] H. W. Lenstra, Jr.. Factoring integers with elliptic curves, Ann. of Math. (2) 126 (1987), no. 3, 649-673. 
[12] Barry Mazur, Arithmetic on curves, Bull. Amer. Math. Soc. (N.S.) 14 (1986), no. 2, 207-259.

[13] Robin J. Y. McLeod and M. Louisa Baart, Geometry and interpolation of curves and surfaces, Cambridge University Press, Cambridge, 1998.

[14] Grace Orzech and Morris Orzech, Plane algebraic curves, Monographs and Textbooks in Pure and Applied Math., vol. 61, Marcel Dekker Inc., New York, 1981, An introduction via valuations.

[15] René Schoof, Elliptic curves over finite fields and the computation of square roots mod p, Math. Comp. 44 (1985), no. 170, 483-494.

[16] Igor R. Shafarevich, Basic algebraic geometry. 1, second ed., Springer-Verlag, Berlin, 1994, Varieties in projective space, Translated from the 1988 Russian edition and with notes by Miles Reid.

[17] Joseph H. Silverman, The arithmetic of elliptic curves, Graduate Texts in Mathematics, vol. 106, Springer-Verlag, New York, 1986.

[18] Joseph H. Silverman and John Tate, Rational points on elliptic curves, Undergraduate Texts in Mathematics, Springer-Verlag, New York, 1992.

[19] Henning Stichtenoth, Algebraic function fields and codes, Universitext, SpringerVerlag, Berlin, 1993. 